\title{
RECONSTRUCTION BASED ON VISIBILITY
}

ALINA SHAIKHET

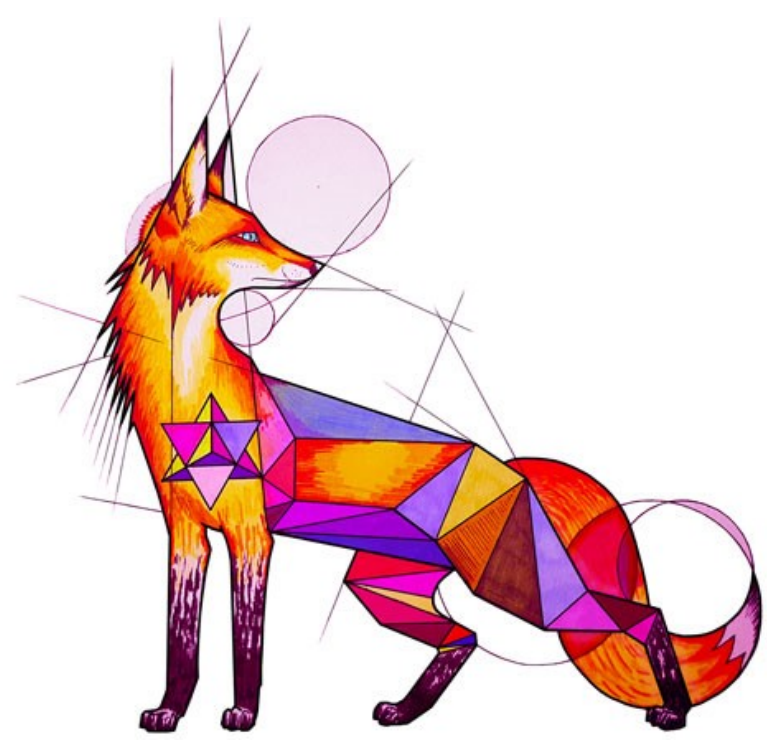

A thesis submitted to the Faculty of Graduate and Postdoctoral Affairs in partial fulfillment of the requirements for the degree of

Doctor of Philosophy in Computer Science

\section{Carleton University}

Ottawa, Ontario, Canada

(C) 2017 Alina Shaikhet 
The thesis investigates geometrical compression of graphs as well as reconstruction of geometrical objects based on a partial information about them.

In the first part we study properties of proximity graphs, such as minimum spanning trees, relative neighbourhood graphs, Gabriel graphs, $\beta$-skeletons and Delaunay triangulations, in the constraint setting.

Given a plane forest $F=(V, E)$ of $|V|=n$ points, we find the minimum set $S \subseteq E$ of edges such that the edge-constrained minimum spanning tree over the set $V$ of vertices and the set $S$ of constraints contains $\mathrm{F}$. We present an $\mathrm{O}(n \log n)$-time algorithm that solves this problem. We generalize this to other proximity graphs in the constraint setting, such as the relative neighbourhood graph, Gabriel graph, $\beta$-skeleton and Delaunay triangulation.

We present an algorithm that identifies the minimum set $S \subseteq E$ of edges of a given plane graph $I=(V, E)$ such that $I \subseteq C_{\beta}(V, S)$ for $1 \leqslant \beta \leqslant 2$, where $C_{\beta}(V, S)$ is the constraint $\beta$-skeleton over the set $V$ of vertices and the set $S$ of constraints. The running time of our algorithm is $O(n)$, provided that the constrained Delaunay triangulation of I is given.

In the second part, we turn our attention to the probing of convex polygons with a wedge. Minimizing the number of probes is one of the main challenges while reconstructing geometric objects with probing devices. In this work, we investigate the problem of using an $\omega$-wedge probing tool to determine the exact shape and orientation of a convex polygon. We assume that we know a point inside the polygon. An $\omega$-wedge consists of two rays emanating from a point called the apex of the wedge and the two rays forming an angle $\omega$. To probe with an $\omega$-wedge, we set the direction that the apex of the probe has to follow, the line $\overrightarrow{\mathrm{L}}$, and the initial orientation of the two rays. A valid $\omega$-probe of a convex polygon $\mathcal{O}$ contains $\mathcal{O}$ within the $\omega$-wedge and its outcome consists of the coordinates of the apex, the orientation of both rays and the coordinates of the closest (to the apex) points of contact between $\mathcal{O}$ and each of the rays.

We present algorithms minimizing the number of probes and prove their optimality. In particular, we show how to reconstruct a convex $n$-gon (with all internal angles of size larger than $\omega$ ) using $2 n-2$ $\omega$-probes; if $\omega=\pi / 2$, the reconstruction uses $2 n-3 \omega$-probes. We show that both results are optimal. Let $N_{B}$ be the number of vertices of $\mathcal{O}$ whose internal angle is at most $\omega$, (we show that $0 \leqslant N_{B} \leqslant 3$ ). We determine the shape and orientation of a general convex n-gon with $N_{B}=1$ (respectively $N_{B}=2$, $N_{B}=3$ ) using $2 n-1$ (respectively $\left.2 n+3,2 n+5\right) \omega$-probes. We prove optimality for the first case. Assuming the algorithm knows the value of $N_{B}$ in advance, the reconstruction of $\mathcal{O}$ with $N_{B}=2$ or $N_{B}=3$ can be achieved with $2 n+2$ probes,- which is optimal.

The third and the fourth problems revolve around the Art Gallery Localization problem.

In the third part we study the problem of placing a set $T$ of broadcast towers in a simple polygon $P$ in order for any point to locate itself in the interior of $P$. Let $V(p)$ denote the visibility polygon of a point $p$, as the set of all points $q \in P$ that are visible to $p$. For any point $p \in P$ : for each tower $t \in T \cap V(p)$ the point $p$ receives the coordinates of $t$ and the Euclidean distance between $t$ and $p$. From this information $p$ can determine its coordinates. We show a tower-positioning algorithm that computes such a set $T$ of size at most $\lfloor 2 n / 3\rfloor$, where $n$ is the size of $P$. This improves the previous upper bound of $\lfloor 8 n / 9\rfloor$ towers [17]. We also show that $\lfloor 2 n / 3\rfloor$ towers are sometimes necessary.

In the fourth part we study the computational complexity of the Art Gallery Localization problem. We show that determining the minimum number of broadcast towers that can localize a point anywhere in a simple polygon P is NP-hard problem. 


\section{ACKNOWLEDGMENTS}

I would not have been able to write this thesis without the help and support of many people.

First of all, I would like to thank my supervisors - Prosenjit Bose, Michiel Smid, and Jean-Lou De Carufel for their invaluable guidance, constant support, encouragement, advice and patience. Thank you for helping me solve so many interesting problems and providing me with many insightful suggestions. I have been extremely lucky to have supervisors who cared so much about my work, and who responded to my questions and queries so promptly.

I would like to thank my thesis committee members for all of their guidance through this process; your discussion, ideas, and feedback have been absolutely invaluable.

Furthermore, I would like to thank all the members and students of the Computational Geometry lab for making it such a nice place. I am very grateful to all the researchers and students who I got to work with during my PhD studies. Working together was always a pleasure, and they taught me more than classes ever could.

Finally, I would like to thank my family and friends for their support during these long, and at times stressful, years. I am especially grateful to my husband Gennady and to my lovely kids Michael and Margarita for their love, support and understanding.

Thank you! 


\section{CONTENTS}

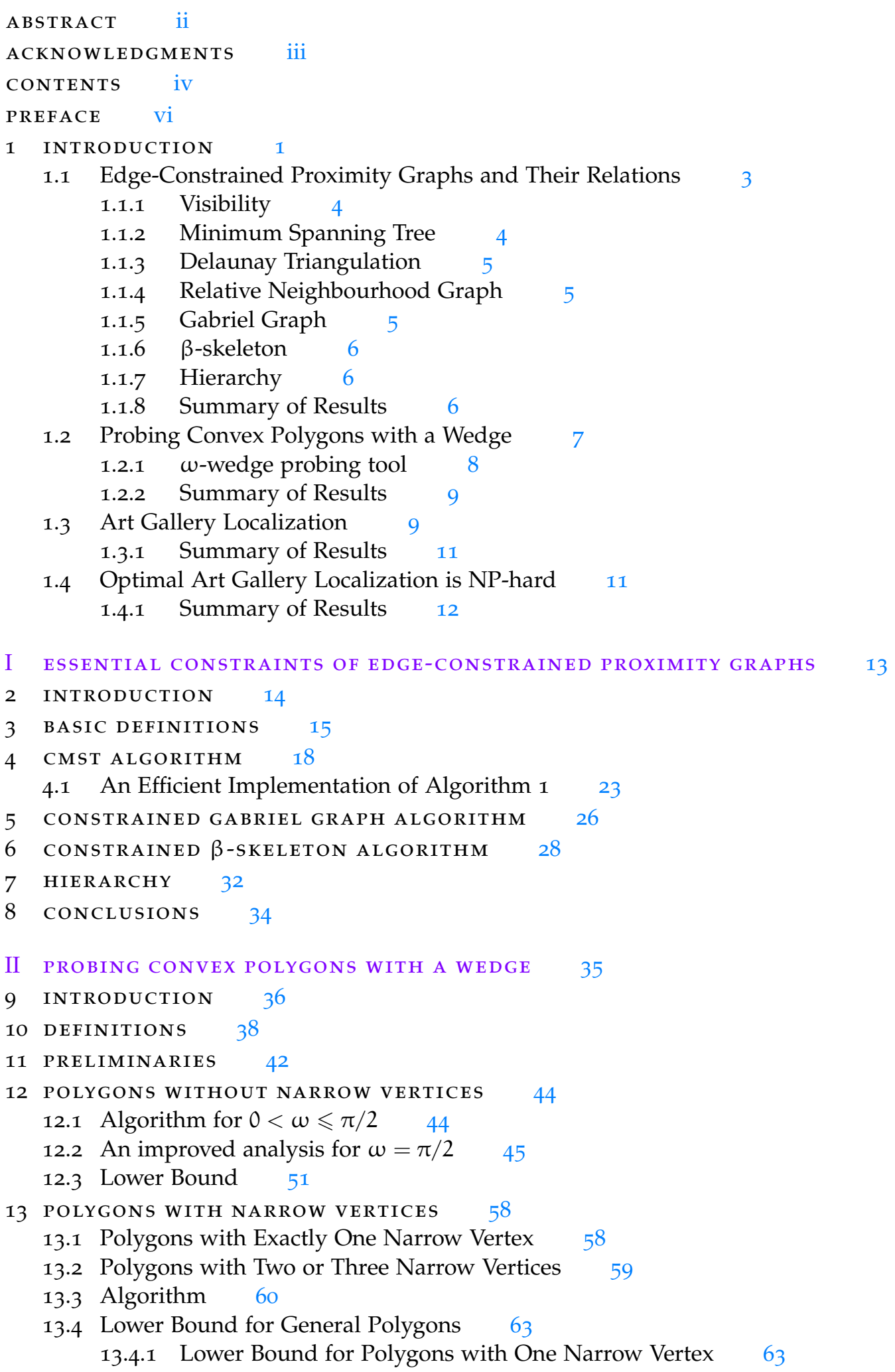


13.4.2 Lower Bound for Polygons with Two or Three Narrow Vertices

14 CONCLUSION AND FUTURE WORK 71

III ART GALLERY LOCALIZATION 73

15 INTRODUCTION 74

16 PRELIMINARIES 76

17 TÓTH'S PARTITION 80

17.1 Case Study 81

17.2 Point-Kernel Problem 82

$17 \cdot 3 n=3 k+2$ and $P^{\prime}$ has no good diagonal dissection $\quad 85$

17.3.1 Adaptation of Tóth's Lemma 3 to our problem 85

17.3.2 Proof of Tóth's Lemma 2 and its adaptation to our problem 86

17.4 Partition algorithm 92

17.5 Counterexample to Tóth's conjecture 93

18 LOCALizATION ALgORITHM 95

19 CONCLUDING REMARKS 96

IV OPTIMAL ART GALLERY LOCALIZATION IS NP-HARD 97

20 INTRODUCTION 98

21 PRELIMINARIES 99

22 OPTIMAL ART GALLERY LOCALIZATION IS NP-HARD IOI

22.1 Literal Pattern 101

22.2 Clause Junction 102

22.3 Variable Pattern 103

22.4 Complete Construction 105

22.5 Construction takes Polynomial Time. 107

23 CONCLUDING REMARKS 112

BIBLIOGRAPHY 113 
This thesis is in "integrated article format" in which each part is based on published papers, conference proceedings, or papers awaiting publication.

- Part I considers the essential constraints of edge-constrained proximity graphs. This part presents the results that have been published in the Journal of Graph Algorithms and Applications (JGAA) [9]. A preliminary version of this paper appeared in the Proceedings of 27th International Workshop, IWOCA 2016, Helsinki, Finland [5]. It was published by Springer in the Lecture Notes in Computer Science (LNCS) series [35].

- Part II deals with the probing convex polygons with a wedge. This part presents the results that have been published in the Journal of Computational Geometry: Theory and Applications (CGTA) [6].

- Part III considers the Art Gallery Localization problem. The results, presented in this part, are submitted to the Journal of Computational Geometry: Theory and Applications (CGTA) [7].

- Part IV further investigates the Art Gallery Localization problem and shows that the optimal Art Gallery Localization in NP-hard. The results, presented in this part, are submitted to the Journal of Computational Geometry: Theory and Applications (CGTA) [8]. 


\section{INTRODUCTION}

This thesis is comprised of four main parts. The first part, Part I, that spans Chapters 2 through 8, deals with the essential constraints of edge-constrained proximity graphs. Part II (Chapters 9 through 14) focuses on probing convex polygons with a wedge. The third part (Part III) found in Chapters 15 through 19 concentrates on worst-case upper and lower bounds of the Art Gallery Localization problem. The last part (Part IV) found in Chapters 20 through 23 shows that the optimal Art Gallery Localization is NP-hard. A brief introduction and summary of each part is given below. The first chapter of each part provides a more detailed introduction.

The common theme of all the four parts of this thesis is reconstruction based on visibility. In the first part we investigate geometric compression of different graphs based on visibility. In the second part we study reconstruction of geometric objects when only partial information about the object is given. The third part deals with reconstruction of point location inside a polygon considering the visibility of a point to localization towers. The last part addresses the computational complexity of the previous localization problem.

Visibility is among the most fundamental topics in Computational Geometry. We say that two points $u$ and $v$ are mutually visible if the open line segment joining them does not intersect the interior of any obstacle.

We study three different approaches to visibility:

- Visibility graphs.

A graph $\mathrm{G}(\mathrm{V}, \mathrm{E})$ is a structure consisting of a set of vertices $\mathrm{V}=\left\{v_{1}, v_{2}, \ldots\right\}$ and a set of edges $E=\left\{e_{1}, e_{2}, \ldots\right\} ;$ each edge $e$ has two endpoints, which are vertices, and they are not necessarily distinct. When the endpoints of edges are always distinct, and no pair of edges have the same two endpoints, the graph is called simple. In this thesis, all graphs will be simple; the set $V$ is always a set of points and the set $E$ is always a set of line segments. In addition, both $V$ and $E$ are assumed to be finite.

A simple polygon $\mathrm{P}$ is usually defined as a collection of $\mathrm{n}$ vertices $v_{1}, v_{2}, \ldots, v_{\mathrm{n}}$ and $\mathrm{n}$ edges $\overline{v_{l} v_{2}}$, $\overline{v_{2} v_{3}}, \ldots, \overline{v_{n-1} v_{n}}, \overline{v_{n} v_{1}}$ such that no pair of nonconsecutive edges share a point and no pair of consecutive edges overlap.

A visibility graph is a graph of inter visible locations, typically for a set of points and obstacles in the Euclidean plane. The vertices of a visibility graph correspond to geometric components (such as vertices of a polygon or vertices of another graph) and two vertices of the visibility graph are connected by an edge if the components are visible to one another, perhaps under some restricted form of visibility. The visibility graph of a simple polygon has as vertex set the vertices of the polygon, and the exterior of the polygon as the only obstacle. Refer to Fig. 1.1a.

Similarly, the visibility graph of a given graph $I=(V, E)$ has $V$ as its vertices and $E$ represents obstacles. Refer to Figures 1.1b and 1.1c. In some settings an obstacle can be an edge of the corresponding visibility graph, and in other settings, obstacles cannot be edges of the corresponding visibility graph.

Visibility graphs may be used to find Euclidean shortest paths among a set of polygonal obstacles in the plane. They can also be applied for planning the motion of a robot among the obstacles. This brings us to another aspect of visibility - robotic vision or sensor touch visibility. 


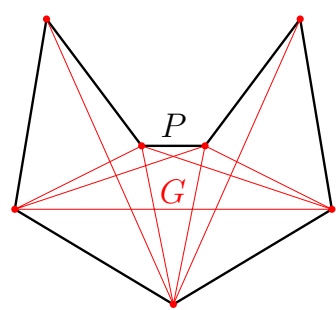

(a)

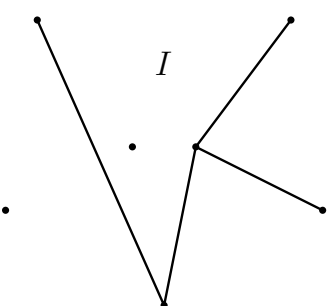

(b)

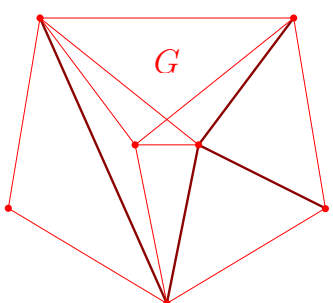

(c)

Figure 1.1: Example of a visibility graph G (highlighted in red). (a) Visibility graph G of a polygon P. The polygon shown in black. (b) Input graph I. (c) Visibility graph G of the given graph I. The edges that belong to both I and $\mathrm{G}$ are shown in dark red.

\section{- Sensor touch visibility.}

Identifying and understanding objects from sensory data is a fundamental problem in robotics and computer vision. For example, a robot equipped with a tactile sensor can touch an object or its environment to learn about it. It is widely used in automated manufacturing. For example, you can check if the shape of the object you manufacture deviates from the blueprint. Tactile sensing is especially important when vision is not useful or is greatly impaired. For example, the object of study may be transparent or may have a reflective surface. Although imaging devices provide a tremendous amount of information, for reasons of economy and robustness, simple sensors are often used. Tactile sensors can be as simple as a whisker. They are cheap and can reach places no camera can.

- Art gallery (or polygon) visibility.

Let $\mathrm{P}$ be a simple polygon. For a point $u$ in $\mathrm{P}$, we let $\mathrm{V}(\mathrm{u})$ denote the visibility polygon of $u$, as the set of all points $q \in P$ that are visible to $u$. Refer to Figure 1.2a. In a similar way we define the visibility polygon of a set of points as follows: $V\left(u_{1}, u_{2}, \ldots, u_{n}\right)=V\left(u_{1}\right) \cup V\left(u_{2}\right) \cup \ldots \cup V\left(u_{n}\right)$. Refer to Figure 1.2b. The visibility polygon of a point inside a simple polygon can be computed in linear time, which is optimal $[25,33,29]$.

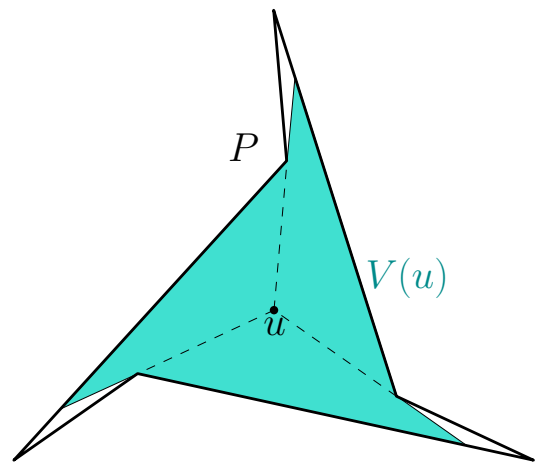

(a)

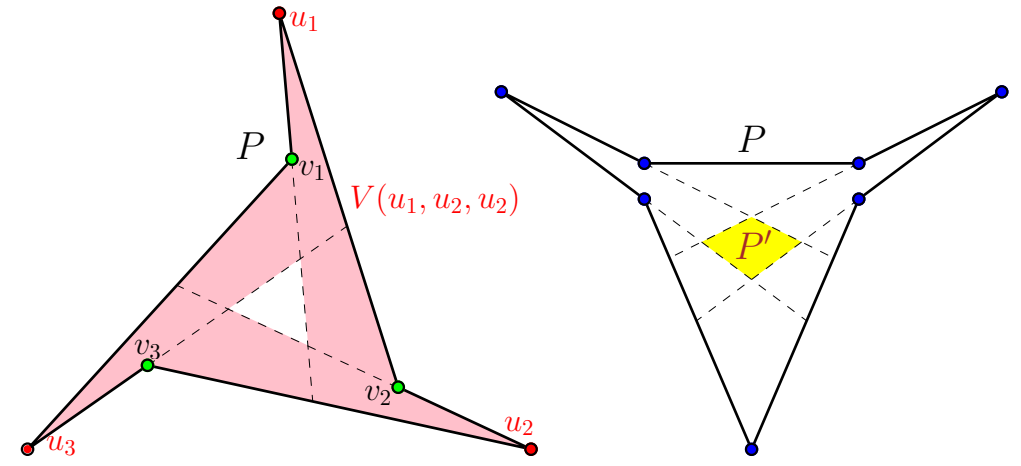

(b)

(c)

Figure 1.2: Examples of visibility polygons, given a simple polygon P. (a) Visibility polygon of the point $u$ is highlighted in turquoise. (b) $V\left(u_{1}, u_{2}, u_{3}\right)$ is shown in pink. (c) Visibility polygon of any point that belongs to $\mathrm{P}^{\prime}$ (highlighted in yellow) is equal to $\mathrm{P}$.

The art gallery problem is a well-studied visibility problem in Computational Geometry. The art gallery is represented by a simple polygon and each guard is represented by a point in the polygon. A set $\mathrm{Q}$ of points is said to guard a polygon $\mathrm{P}$ if $\mathrm{V}(\mathrm{Q})=\mathrm{P}$. For example, the guard, 
positioned at point $u$ (refer to Fig. 1.2a) cannot guard the whole gallery $P$ because $V(u) \neq P$. Consider an example of Fig. 1.2c. A single guard, positioned anywhere inside the polygon $\mathrm{P}^{\prime}$ (highlighted in yellow) can guard the whole art gallery $\mathrm{P}$. This example also shows that point guards (guards that can be positioned anywhere in $\mathrm{P}$ ) are more powerful than vertex guards (guards whose positions are restricted to the vertices of $\mathrm{P}$ ). This gallery can be guarded with a single point guard, but requires at least 2 vertex guards.

It is important to notice that if a set of guards can see the whole boundary of the gallery $\mathrm{P}$, it does not mean that they also can see the complete interior of P. Refer to Fig. 1.2b. The guards positioned at $u_{1}, u_{2}$ and $u_{3}$ can see the boundary of $P$, but not its complete interior. The visibility polygon of the triple of guards is highlighted in pink. Notice that $V\left(u_{1}, u_{2}, u_{3}\right) \neq P$.

\section{I EDGE-CONSTRAINED PROXIMITY GRAPHS AND THEIR RELATIONS}

The notation $e=\overline{u v}$ means that the edge $e$ is incident on vertices $u$ and $v$. In this case we also say that e connects vertices $u$ and $v$, or that vertices $u$ and $v$ are adjacent.

A path, $Q$, is a sequence of vertices and edges interweaved in the following way: $Q$ starts with a vertex, say $v_{0}$, followed by an edge $e_{1}$ incident to $v_{0}$, followed by the other endpoint $v_{1}$ of $e_{1}$, and so on. If no vertex or edge is repeated, then the path is simple. We assume that $Q$ is finite and thus it ends with a vertex, say $v_{l}$. We call $v_{0}$ the start-vertex of $\mathrm{Q}$ and $\nu_{\mathrm{l}}$ the end-vertex of $\mathrm{Q}$. The number of edges appearing in $\mathrm{Q}$, is the length of $\mathrm{Q}$.

A cycle is a path of finite and non-zero length in which the start and end vertices are identical. A cycle is simple, if there is no repetition of an edge or a vertex except that the start vertex is identical to the end vertex.

If for every two vertices $u$ and $v$ of a graph $G$, there is a path that starts at $u$ and ends at $v$, then $G$ is said to be connected.

A proximity graph (also referred as neighbourhood graph) is a graph in which two vertices are connected by an edge if and only if the vertices satisfy particular geometric requirements.

A planar graph is a graph that can be embedded in the plane, in such a way that its edges intersect only at their endpoints.

A triangulation is a planar graph where each face is a triangle (a cycle of three edges). The Delaunay triangulation for a set $\mathrm{V}$ of points in a plane is a triangulation $\mathrm{DT}(\mathrm{V})$ such that no point in $\mathrm{V}$ is inside the circumcircle of any triangle in DT(V). Delaunay triangulations maximize the minimum angle of all the angles of the triangles in the triangulation [2].

This research was inspired by topics in geometric compression. The most well studied objects of geometric compression are meshes and terrain models. Compression algorithms are required to compress both the geometry data and the connectivity data. The geometry is represented by the set of vertices (or coordinates) of the mesh. The connectivity data (also referred to as the topology) is the vertex/triangle adjacency list. In a naive representation, the connectivity data is about twice as large as the geometry data. In some cases the topology can be computed from the geometry. For example, some terrain models are obtained by using the Delaunay triangulation. Mesh compression algorithms are normally required to use a lossless compression of the connectivity data. Unfortunately, not all triangulations are Delaunay triangulations, and encoding a non Delaunay triangulation must include some topology. Our interest in the topic originated from the paper by Devillers et al. [16]. A method, presented in that paper, consists of finding the fewest number of segments such that the constrained Delaunay triangulation is identical to any given input triangulation. In particular, Devillers et al. investigate how to compute the minimum set $S$ of edges of a given planar triangulation $T=(V, E)$, such that $T$ is a constrained Delaunay triangulation of the graph $(V, S)[16]$. They show that $S$ and $V$ is the 
only information that needs to be stored. The graph $T$ can be successfully reconstructed from $S$ and $\mathrm{V}$. They also used their method of compression on real data sets (such as terrain models and meshes). Their experiments show that the size of $S$ is less than $3.4 \%$ of the total number of edges of $T$, which yields a very effective compression of the triangulation.

Our interest was to broaden this research and investigate geometric compression of other proximity graphs. We study minimum spanning trees, relative neighbourhood graphs, Gabriel graphs and $\beta$ skeletons for $1 \leqslant \beta \leqslant 2$. In this chapter we give a definition of each of those graphs in the constraint setting.

Problem Definition. Let $I=(V, E)$ be an arbitrary plane graph. Each pair of points $u, v \in V$ is associated with a neighbourhood defined by some property $\mathrm{P}(\mathrm{u}, v, \mathrm{I})$. The property depends on the proximity graph we study. An edge-constrained proximity graph $G_{P}(I)$ defined by the property $P$ is a graph with a set $V$ of vertices and a set $E_{P}$ of edges such that $\overline{u v} \in E_{P}$ if and only if $\overline{u v} \in E$ or $\overline{u v}$ satisfies $\mathrm{P}(\mathrm{u}, v, \mathrm{I})$. Please refer to Fig. 3.2 (in Chapter 3 ) which shows a relationship between the input graph and its edge-constraint proximity graphs.

\subsubsection{Visibility}

Two vertices $u$ and $v$ are visible to each other with respect to $E$ provided that $\overline{u v} \in E$ or the line segment $\overline{u v}$ does not intersect the interior of any edge of $E$.

Given a plane graph $I=(V, E)$, the visibility graph of $I$ is the graph $V G(I)=\left(V, E^{\prime}\right)$ such that $E^{\prime}=\{(u, v): u, v \in V, u$ and $v$ are visible to each other with respect to E\}. Intuitively, the graph I represents a set of obstacles in Euclidean space. The nodes of VG(I) are the vertices of I, and there is an edge between vertices $u$ and $v$ if they can see each other, that is, if the line segment $\overline{u v}$ does not intersect the interior of any obstacle in I. Notice that we assume that the endpoints of an obstacle edge see each other. Since I is a plane graph, it is natural to assume that the obstacle edges form a subset of the edges of $\mathrm{VG}(\mathrm{I})$, and thus $\mathrm{I} \subseteq \mathrm{VG}(\mathrm{I})$.

The Euclidean visibility graph EVG(I) is the visibility graph of $\mathrm{I}$, where each edge $\overline{u v}(u, v \in V)$ is assigned the weight $w(u, v)$ that is equal to the Euclidean distance between $u$ and $v$.

Notice that VG(I) is a simple and unweighted graph, while EVG(I) is a simple weighted graph.

The constrained visibility graph CVG(I) is the Euclidean visibility graph of I, where each edge of I is assigned the weight 0 . Notice that every other edge has weight equal to its Euclidean length.

\subsubsection{Minimum Spanning Tree}

We say that graph $G$ is cycle-free if there are no simple cycles in G. The graph $G$ is called a tree if it is connected and cycle-free. If there are $n$ vertices in the graph, then each spanning tree has $n-1$ edges. A forest is cycle-free graph that is not necessarily connected. Forests therefore consist only of (possibly disconnected) trees.

A subgraph of $G$ that contains all of its vertices and is a tree is called a spanning tree of G. A minimum spanning tree is a spanning tree of a connected, undirected graph, that connects all the vertices together with the minimum total weight (or length) for its edges.

We use the notation MST $(G)$ to refer to a minimum spanning tree of the graph G. We assume that each edge of $G$ has weight equal to its Euclidean length, unless the edge was specifically assigned the weight 0 by our algorithm. If none of the edges of $G$ were assigned the weight 0 then $M S T(G)$ is a Euclidean minimum spanning tree of $\mathrm{G}$.

Given a plane forest $\mathrm{F}=(\mathrm{V}, \mathrm{E})$, the constrained minimum spanning tree $\mathrm{CMST}(\mathrm{F})$ is the minimum spanning tree of CVG(F). Refer to Fig. 3.2b. 
There are many algorithms that find $\mathrm{MST}(\mathrm{G})$. One of the classical ones was written by Joseph Kruskal in 1956. Kruskal's algorithm is an MST algorithm which finds an edge of the least possible weight that connects any two trees in the forest [15]. Its running time is $\mathrm{O}(|\mathrm{E}| \log |\mathrm{V}|)$, where $\mathrm{G}=(\mathrm{V}, \mathrm{E})$. Notice that, since each edge of a plane forest $F$ has weight zero in $C V G(F)$, by running Kruskal on $\mathrm{CVG}(\mathrm{F})$, we get $\mathrm{F} \subseteq \mathrm{CMST}(\mathrm{F})$. Notice also that if $\mathrm{F}$ is a plane tree, then $\mathrm{F}=\mathrm{CMST}(\mathrm{F})$.

Minimum spanning trees are a well-studied area of research. But, to the best of our knowledge, edgeconstrained MSTs have not been considered before. We study the problem of finding the minimum set $S \subseteq E$ of constraint edges from a given plane forest $F=(V, E)$ such that the edge-constrained MST over the set $V$ of vertices and the set $S$ of constraints contains $F$. We give an $O(n \log n)$-time algorithm that solves this problem.

\subsubsection{Delaunay Triangulation}

A subset $Q$ of the plane is called convex if and only if for any pair of points $q_{1}, q_{2} \in Q$ the line segment $\overline{\mathrm{q}_{1} \mathrm{q}_{2}}$ is completely contained in $\mathrm{Q}$. The convex hull $\mathrm{CH}(\mathrm{Q})$ of a set $\mathrm{Q}$ is the smallest convex set that contains $\mathrm{Q}$. To be more precise, it is the intersection of all convex sets that contain $\mathrm{Q}$.

Let $G$ be a triangulation, $\overline{\nu_{1} v_{2}}$ be an edge in $G$ (but not an edge of the convex hull of $G$ ), and $\triangle\left(v_{1}, v_{2}, v_{3}\right)$ and $\triangle\left(v_{1}, v_{2}, v_{4}\right)$ be the triangles adjacent to $\overline{v_{1} v_{2}}$ in G. We say that $\overline{v_{1} v_{2}}$ is a locally Delaunay edge if the circle through $\left\{v_{1}, v_{2}, v_{3}\right\}$ does not contain $v_{4}$ or equivalently if the circle through $\left\{v_{1}, v_{2}, v_{4}\right\}$ does not contain $v_{3}$. Every edge of the convex hull of $\mathrm{G}$ is also considered to be locally Delaunay. [16].

Given a plane graph $\mathrm{I}=(\mathrm{V}, \mathrm{E})$, the constrained Delaunay triangulation $\mathrm{CDT}(\mathrm{I})$ is the unique triangulation of $V$ such that each edge is either in E or locally Delaunay. It follows that $I \subseteq C D T(I)$. Refer to Fig. 3.2f.

\subsubsection{Relative Neighbourhood Graph}

Toussaint introduced the relative neighbourhood graph (RNG) in 1980 and showed its application in pattern recognition [45]. To give the definition of RNG we first need to define a lune. Let $D(x, r)$ denote an open disk centered at a point $x$ with radius $r$, i.e., $D(x, r)=\{y: \operatorname{dist}(x, y)<r\}$, where $\operatorname{dist}(x, y)$ is the Euclidean distance between $x$ and $y$. Let $\mathrm{L}_{\mathfrak{u}, v}=\mathrm{D}(\mathrm{u}, \operatorname{dist}(\mathrm{u}, v)) \cap \mathrm{D}(v, \operatorname{dist}(u, v)) ; \mathrm{L}_{\mathfrak{u}, v}$ is called a lune.

Given a set $\mathrm{V}$ of points, the Relative Neighbourhood graph of $\mathrm{V}, \mathrm{RNG}(\mathrm{V})$, is the graph with vertex set $V$ and the set $E$ of edges defined as follows: $\overline{u v} \in E$ if and only if $L_{u, v} \cap V=\emptyset$.

Jaromczyk, Kowaluk and Yao showed that RNG of a set $V$ of points can be constructed from the Delaunay triangulation of $\mathrm{V}$ in linear time [28].

Given a plane graph $\mathrm{I}=(\mathrm{V}, \mathrm{E})$, the constrained Relative Neighbourhood graph, $\mathrm{CRNG}(\mathrm{I})$, is defined as the graph with vertices $V$ and the set $E^{\prime}$ of edges such that each edge $e=\overline{u v}$ is either in $E$ or, $u$ and $v$ are visible to each other and $\mathrm{L}_{u, v}$ does not contain points in $\mathrm{V}$ which are visible from both $u$ and $v$. It follows that $\mathrm{I} \subseteq \mathrm{CRNG}(\mathrm{I})$. Refer to Fig. 3.2C.

\subsubsection{Gabriel Graph}

Gabriel graphs were introduced by Gabriel and Sokal for the purpose of geographic variation analysis [22]. The Gabriel graph of a set $V$ of points is the graph with vertex set $V$ in which any points $u$ and $v$ of $V$ are connected by an edge if they are distinct and the closed disk with $\overline{u v}$ as a diameter contains no other point of $\mathrm{V}$. 
The edge $\overline{u v}$ of the plane graph $G=(\mathrm{V}, \mathrm{E})$ is said to be locally Gabriel if the vertices $u$ and $v$ are visible to each other and the circle with $\overline{u v}$ as a diameter does not contain any points in $V$ which are visible from both $u$ and $v$. Refer to Figure 3.1.

Given a plane graph $I=(V, E)$, the constrained Gabriel graph $C G G(I)$ is defined as the graph with vertices $V$ and the set $E^{\prime}$ of edges such that each edge is either in $E$ or locally Gabriel. It follows that $\mathrm{I} \subseteq \mathrm{CGG}(\mathrm{I})$. Refer to Fig. 3.2e.

\subsection{6 $\beta$-skeleton}

$\beta$-skeletons were defined by Kirkpatrick and Radke in [30]. Those graphs represent a parametrized family of neighbourhood graphs. The neighbourhood $U_{u, v}(\beta)$ is defined for any fixed $\beta(1 \leqslant \beta<\infty)$ as the intersection of two disks:

$$
\mathrm{u}_{\mathrm{u}, v}(\beta)=\mathrm{D}\left(\left(1-\frac{\beta}{2}\right) \mathrm{u}+\frac{\beta}{2} v, \frac{\beta}{2} \operatorname{dist}(u, v)\right) \cap \mathrm{D}\left(\left(1-\frac{\beta}{2}\right) v+\frac{\beta}{2} u, \frac{\beta}{2} \operatorname{dist}(u, v)\right)
$$

Refer to Figure 7.1.

Given a set $V$ of points in the plane, the (lune-based) $\beta$-skeleton of $V$, denoted $G_{\beta}(V)$ is the graph with vertex set $V$ and edge set defined as follows: $\overline{u v}$ is an edge if and only if $U_{u, v}(\beta) \cap V=\emptyset$.

Relative neighbourhood and Gabriel graphs are special cases of $\beta$-skeletons. In particular, the 1skeleton corresponds to the Gabriel graph and the 2-skeleton corresponds to the relative neighbourhood graph. To be specific $R N G(V)=G_{2}(V)$ and $G G(V)=G_{1}(V)$.

Given a plane graph $I=(V, E)$, the constrained $\beta$-skeleton of $I, C_{\beta}(I)$ is the graph with vertex set $V$ and edge set $E^{\prime}$ defined as follows: $e=\overline{u v} \in E^{\prime}$ if and only if $e \in E$ or $u$ and $v$ are visible to each other and $U_{u, v}(\beta)$ does not contain points in $V$ which are visible from both $u$ and $v$. Refer to Fig. 3.2d.

\subsubsection{Hierarchy}

Toussaint was one of the first who established a nested hierarchy over proximity graphs [45]: for a fixed set of points and for any $1 \leqslant \beta \leqslant 2$, the following is true:

$$
\mathrm{MST} \subseteq \mathrm{RNG} \subseteq \mathrm{G}_{1 \leqslant \beta \leqslant 2} \subseteq \mathrm{GG} \subseteq \mathrm{DT} .
$$

We show that proximity graphs in the constraint setting preserve the above hierarchy. Moreover, we show that the minimum set of constraints required to reconstruct a given planar graph (as a part of each of those proximity graphs) form an inverse hierarchy (refer to Chapter 7).

\subsubsection{Summary of Results}

For different types of proximity graphs, given a plane graph $I=(V, E)$, we compute the minimum set $S \subseteq E$ such that the constrained proximity graph, with constraints $S$, contains $I$. In this research we show that the proximity graph that contains I can be successfully reconstructed from $\mathrm{V}$ and $\mathrm{S}$.

We present an $O(n \log n)$-time algorithm that identifies the minimum set $S \subseteq E$ of constraint edges given a plane forest $\mathrm{F}=(\mathrm{V}, \mathrm{E})$ of $|\mathrm{V}|=\mathrm{n}$ points in the plane, such that the edge-constrained MST over the set $V$ of vertices and the set $S$ of constraints contains $F$. We show some observations concerning constrained MST and give worst-case examples. In particular, in Lemma 4.2 we prove that $\operatorname{CMST}(\mathrm{V}, \mathrm{S}) \subseteq \mathrm{VG}(\mathrm{F})$. Refer to Chapter 4 .

In Chapter 6 we present an algorithm that identifies the minimum set $S$ of edges of a given planar graph $I=(V, E)$ such that $I \subseteq C_{\beta}(V, S)$ for $1 \leqslant \beta \leqslant 2$, where $C_{\beta}(V, S)$ is the constraint $\beta$-skeleton 
on the set $V$ of vertices and set $S$ of constraints. The running time of the algorithm is $O(n)$, provided that the constrained Delaunay triangulation of I is given.

We investigate the special case of constrained $\beta$-skeletons - constrained Gabriel graphs (1-skeletons). Although the algorithm for general $\beta$-skeletons (given in Chapter 6) can be successfully applied to constrained Gabriel graphs, we give another, significantly simpler algorithm that requires only local information about an edge in question.

We show the hierarchy of the constrained proximity graphs together with the hierarchy of the minimum sets of constraints in Chapter 7 .

These results have been published in the Journal of Graph Algorithms and Applications (JGAA) [9]. A preliminary version of this work appeared in the Proceedings of 27th International Workshop, IWOCA 2016, Helsinki, Finland [5]. It was published by Springer in the Lecture Notes in Computer Science (LNCS) series [35].

\subsection{PROBING CONVEX POLYGONS WITH A WEDGE}

Geometric probing is an active and well developed area of research. It is a branch of Computational Geometry looking into how to determine shapes of unknown geometric objects by using special measurements known as probes. Geometric probing was introduced by Cole and Yap [14] in 1983. Since then many probing tools, together with reconstruction algorithms, have been developed - finger probes [14], hyperplane (or line) probes [18, 34], diameter probes [38], x-ray probes [19, 23], histogram (or parallel x-ray) probes [36], half-plane probes [42], composite probes [10, 34, 40] among others. More sophisticated sensing devices can be constructed by considering more than one type of probe. See the review paper of Skiena [41], discussing the methodology as well the challenges of geometric probing.

Various probing schemes along with upper and lower bounds on performance are summarized in Table 1.1. Notice, that those results hold only for convex polygons. A simple polygon is convex if all its interior angles are less than or equal to $180^{\circ}$. For certain probing models, extensions to more general objects are impossible.

\begin{tabular}{l|c|c|c|c}
\hline \hline Probing Tool & Notes & Lower & Upper & References \\
\hline \hline finger & & $3 n-1$ & $3 n$ & {$[14]$} \\
\hline hyperplane/line & & $3 n+1$ & $3 n+1$ & {$[34]$} \\
\hline diameter & Reconstruction is not possible & $*$ & $*$ & {$[38]$} \\
\hline x-ray & & $2 n$ & $5 n+19$ & {$[19]$} \\
\hline half-plane & & $2 n$ & $7 n+7$ & {$[42]$} \\
\hline \multirow{4}{*}{$\omega$-wedge } & $\omega=\pi / 2, N_{B}=0$ & $2 n-3$ & $2 n-3$ & \\
& $0<\omega<\pi / 2, N_{B}=0$ & $2 n-2$ & $2 n-2$ & \\
& $0<\omega \leqslant \pi / 2, N_{B}=1$ & $2 n-1$ & $2 n-1$ & our result \\
& $0<\omega \leqslant \pi / 2, N_{B}=2$ & $2 n+2$ & $2 n+3$ & \\
& $0<\omega \leqslant \pi / 2, N_{B}=3$ & $2 n+2$ & $2 n+5$ & \\
\hline
\end{tabular}

Table 1.1: Summary of results for reconstructing convex polygons together with lower and upper bounds. $N_{B}$ stands for the number of angles whose size is at most $\omega$ in the convex polygon. 
Another closely related area, called geometric testing, studies a verification problem: find a set of probes to determine if a given set of geometric objects contains one which is equivalent to a certain query object (see [39] for a survey).

Probes are considered to be expensive. Thus, for a given probing tool, we seek to reconstruct an object with as few probes as possible. Much effort has gone to finding an algorithm with sufficiently tight upper and lower bounds or, ultimately, an optimal algorithm.

\subsection{1 w-wedge probing tool}

We introduce a new probing tool - an $\omega$-wedge. It consists of two rays emanating from a point called the apex of the wedge, forming an angle $\omega$, where $\omega$ is a fixed real number with $0<\omega \leqslant \pi / 2$. Refer to Fig. 10.1a. Given a convex polygon $\mathcal{O}$ in the plane, the goal is to probe $\mathcal{O}$ to determine its exact shape and orientation with a minimum number of probes. We assume that the object $\mathcal{O}$ is fixed and we know one point $x \in \mathcal{O}$. To probe a convex polygon $\mathcal{O}$ we choose a directed line $\overrightarrow{\mathrm{L}}$ and using the point $x$, we position the apex of the $\omega$-wedge on $\overrightarrow{\mathrm{L}}$ such that $\mathcal{O}$ and the positive direction of $\overrightarrow{\mathrm{L}}$ are contained in the wedge. Refer to Fig. 10.1b. It is important to choose $\overrightarrow{\mathrm{L}}$ in such a way that $\overrightarrow{\mathrm{L}}$ intersects the interior of $\mathcal{O}$. Otherwise the probe can miss the object (refer to Fig. 10.1c). Initially, we position the wedge such that $\mathcal{O}$ does not touch the rays of the wedge. Informally, imagine the $\omega$-wedge moving from its initial position along and in the direction of $\overrightarrow{\mathrm{L}}$ until both rays contact $\mathcal{O}$ in which case the wedge cannot move any further. A valid $\omega$-probe of a convex polygon $\mathcal{O}$ is a placement of an $\omega$-wedge, such that $\mathcal{O}$ is contained in the wedge and touches both of its rays. An outcome of a valid $\omega$-probe consists of the coordinates of the apex, the orientation of both rays and the coordinates of the points of contact between $\mathcal{O}$ and each of the rays closest to the apex.

The main difficulty we experienced with our probing tool is when the convex polygon contains so called narrow vertices. A vertex of the convex polygon $\mathcal{O}$ is called a narrow vertex if its internal angle is at most $\omega$. If the ray in the direction of an $\omega$-probe enters $\mathcal{O}$ via a narrow vertex $v_{\mathrm{B}}$, then the apex of this probe is on a vertex $v_{\mathrm{B}}$ of $\mathcal{O}$. Refer to Figure 10.2c. In this case the rays of the wedge may or may not touch $\mathcal{O}$. Luckily, there can be no more than 3 vertices on the polygon where this happens (refer to Observation 11.1).

Our probing tool is similar to finger probing [14]. A finger probe is defined to be a directed line $\overrightarrow{\mathrm{L}}$. Informally, imagine a point moving along and in the direction of $\overrightarrow{\mathrm{L}}$ until it contacts $\mathcal{O}$. In our research the point is equipped with two rays. The movement of the apex $q$ along $\overrightarrow{\mathrm{L}}$ terminates when both rays contact $\mathcal{O}$ (while the apex does not necessarily touch $\mathcal{O}$ ). Our model of probing achieves better results than finger probing (refer to Table 1.I). It is mainly because the outcome of a valid $\omega$-probe contains one or two points of contact with $\mathcal{O}$ (which are vertices of $\mathcal{O}$ ), while in finger probing the outcome of a successful probe is a single point (not necessarily a vertex of $\mathcal{O}$ ). Moreover, almost every edge of a polygon, reconstructed via finger probing, contains two points of contact in its interior. In our model only one probe is sufficient to verify an edge.

The $\omega$-wedge probing tool should not be confused with diameter or "parallel-jaw gripper" model of probing [38], where the shape of a polygon is determined from a sequence of projections. It was shown by Rao and Goldberg that reconstruction of $\mathcal{O}$ with a diameter probing tool is not possible, because for a given set of diameter measurements, there is an infinite set of polygonal shapes consistent with these measurements [38]. The most crucial difference between our probing tool and the "parallel-jaw gripper" is that a valid $\omega$-probe returns points of contact with $\mathcal{O}$, while a diameter probe does not, it returns only the distance between the supporting lines.

Our probing tool generalizes previous work (see e.g., [4], [21]). The method of angular probing was inspired by problems on enclosing triangles [4], namely searching for all $\omega$-angle triangles of minimum area which enclose a given set of points in the plane. 
The $\omega$-wedge probing tool is quite simple and cheap compared to other probing devices such as x-ray or histogram. It can be manufactured from two tactile whisker-like sensors and does not require complicated software.

\subsubsection{Summary of Results}

In this research, we present an $\omega$-wedge probing tool together with optimal probing algorithms for convex polygons with no narrow vertices or when at most one angle of $\mathcal{O}$ is smaller then $\omega$. We present an algorithm that reconstructs a convex n-gon with all internal angles of size bigger than $\omega$ using $2 n-2 \omega$-probes (for $0<\omega<\pi / 2$ ). When $\omega=\pi / 2$, the reconstruction uses $2 n-3 \omega$-probes. We prove optimality for both cases. We show that $2 n-1$ probes are necessary and sufficient to reconstruct a convex polygon with exactly one vertex whose internal angle is at most $\omega$. When the polygon has 2 or 3 angles smaller than or equal to $\omega$, our results are almost optimal. In particular, for polygons with exactly 2 (respectively, 3 ) angles of size at most $\omega$, we show a reconstruction strategy that uses $2 n+3$ (respectively, $2 n+5) \omega$-probes, while $2 n+2$ probes are necessary. The main reason why our results are not tight is due to the fact that the number of narrow vertices is assumed to be unknown apriori. Knowing this extra information about polygon $\mathcal{O}$ beforehand improves our strategy and brings down the upper bounds. The ideas on how to do this are given in Chapter 14 .

Our results are summarized in Table I.I, where you can compare them to other results in the field. Notice that the $\omega$-wedge probing tool requires less probes for reconstruction compared to other devices. Moreover, our results are optimal or near optimal. Our reconstruction strategies are adaptive, i.e., the choice of parameters for each probe depends on all previous outcomes. We also prove lower bounds for all of our algorithms using adversarial arguments. These results were published in the Journal of Computational Geometry: Theory and Applications (CGTA) [6].

The organization of Part II of the thesis is as follows. In Chapter 9 we give introduction to geometric probing. Chapter 1o contains some definitions related to our probing method. In Chapter II we give some observations, review known facts and prove some fundamental properties related to $\omega$-probing. We concentrate on the reconstruction of convex polygons in Chapters 12 and 13. We present an optimal algorithm for reconstructing convex polygons that do not have narrow vertices in Chapter 12. Reconstruction of general convex polygons together with the discussion on upper and lower bounds on the performance are given in Chapter 13. Conclusions and some ideas about future work can be found in Chapter 14 .

\subsection{ART GALLERY LOCALIZATION}

In 1973, Victor Klee posed the problem of determining the minimum number of guards sufficient to cover (or guard) the interior of an n-wall art gallery. It was shown by Chvátal that $\lfloor n / 3\rfloor$ guards are always sufficient and sometimes necessary [13]. This result has become known as "Chvatal's Art Gallery Theorem". This theorem has since been extended in several directions, one of which is directly applied to this thesis - partitioning algorithms. Instead of directly solving the art gallery problem for a given simple polygon $\mathrm{P}$, we can partition $\mathrm{P}$ into a subset of subpolygons that are "easy" to guard.

There are numerous variations of the original art gallery problem. In some versions guards are restricted to the perimeter, or to the vertices of the polygon, in other versions there are restrictions applied to the polygon or to the guards themselves (like restricted field of vision). This research combines the art gallery problem with trilateration. Trilateration is the process of determining absolute or relative locations of points by measurement of distances, using the geometry of the environment. Trilateration does have practical applications in surveying and navigation, including global positioning systems (GPS). Every GPS satellite transmits information about its position and the current time at regular intervals. These signals are intercepted by a GPS receiver, which calculates how far away each 
satellite is based on how long it took for the messages to arrive. GPS receivers take this information and use trilateration to calculate the user's location.

We address the problem of placing broadcast towers (distance measuring guards) in a simple polygon $\mathrm{P}$ in order for a point in $\mathrm{P}$ (let us call it an agent) to locate itself. Towers can be defined as points which can transmit their coordinates together with a time stamp to other points in their visibility region. The agent receives messages from all the towers that belong to its visibility region. Given a message from the tower $t$, the agent can determine its distance to $t$. We use trilateration as the process, during which the agent can determine its absolute coordinates. Receiving a message from one tower only will not be sufficient for the agent to locate itself (unless the agent and the tower are at the the same location). In two-dimensional geometry, it is known that if a point lies on two circles, then the circle centers and the two radii provide sufficient information to narrow the possible locations down to two. Additional information may narrow the possibilities down to one unique location. Refer to Figures 16.1 and 21.1.

In relation to GPS systems, towers can be viewed as GPS satellites, while agents (query points interior to the polygon) can be compared to GPS receivers. Naturally, we would like to minimize the number of towers.

Let $P$ be a simple polygon in general position (no three vertices are collinear) having a total of $n$ vertices on its boundary (denoted by $\partial \mathrm{P})$. A star-shaped polygon is a polygon that contains a point from which the entire polygon boundary (and interior) is visible. Recall that for $u \in P$, we let $V(u)$ denote the visibility polygon of $u$, as the set of all points $q \in P$ that are visible to $u$. Notice that $V(u)$ is a star-shaped polygon contained in $\mathrm{P}$ and $\mathrm{u}$ belongs to its kernel (the set of points from which all of $\mathrm{V}(\mathrm{u})$ is visible). For example, the polygon $\mathrm{P}^{\prime}$ (highlighted in yellow on Fig. 1.2c) is a kernel of P. It also means that the polygon P given on Fig. $1.2 \mathrm{C}$ ) is star-shaped.

Problem Definition: Let $\mathrm{T}$ be a set of points (called towers) in $\mathrm{P}$ satisfying the following properties. For every point $p \in P$ : for each $t \in T \cap V(p)$, the point $p$ receives the coordinates of $t$ and can compute the Euclidean distance between $t$ and $p$, denoted $d(t, p)$. From this information, $p$ can determine its coordinates. We consider the following problems:

1. Design an algorithm that, on any input polygon $\mathrm{P}$ in general position, computes a "small" set $\mathrm{T}$ of towers, such that an agent can localize itself anywhere in P.

2. Design a localization algorithm.

We show how to compute such a set $T$ of size at most $\lfloor 2 n / 3\rfloor$ by modifying the polygon partition method introduced by Tóth [44]. Tóth showed that any simple polygon with $n$ vertices can be guarded by $\lfloor n / 3\rfloor$ point guards whose range of vision is $180^{\circ}$. However, Tóth assumed that his partition method creates subpolygons whose vertices are in general position. We believe this assumption is too strong. We strengthened the work [44] by lifting the assumption. We reproved Tóth's result and showed how to use his method with respect to our problem. In addition, we found and fixed several mistakes in [44]. Refer to Chapter 17. It is important to notice that we assume that the input polygon is in general position, while non-general position may occur for subpolygons of the partition. We show that each $180^{\circ}$-guard $\mathrm{g}$ can be replaced with a pair of towers close to $\mathrm{g}$. We embed the orientation of the $180^{\circ}$-guard into the coordinates of the towers. That is, we specify to which side of the line through the pair of towers their primary localization region resides. We call it the parity trick. The localization algorithm is allowed to use this information.

Our result of $\lfloor 2 n / 3\rfloor$ towers is an improvement over the previous upper bound of $\lfloor 8 n / 9\rfloor$ towers given by Dippel and Sundaram [17]. Their approach is to decompose the polygon into at most $\lfloor n / 3\rfloor$ fans. A polygon $\mathrm{P}^{\prime}$ is a fan if there exist a vertex $u$, such that for every other vertex $v$ not adjacent to $\mathrm{u}, \overline{\mathrm{uv}}$ is a diagonal of $\mathrm{P}^{\prime}$. In each fan with fewer than 4 triangles Dippel and Sundaram position a pair of towers on an edge of the fan; every fan with 4 or more triangles receives a triple of towers in its kernel. In a classical trilateration, the algorithm for locating an agent knows the coordinates of the 
towers that can see $p$ together with distances between $p$ and the corresponding towers. However, the localization algorithm presented in [17] requires a lot of additional information, such as a map of the polygon, the decomposition into fans, the coordinates of all towers, and even the list of towers that do not see the agent. Our localization algorithm has no information about P. It receives as an input only the coordinates of the towers that can see $p$ together with their distances to $p$. In addition our algorithm is empowered by the knowledge of the parity trick. When only a pair $t_{1}, t_{2}$ of towers can see $p$ then the coordinates of the towers together with the distances $d\left(t_{1}, p\right)$ and $d\left(t_{2}, p\right)$ provide sufficient information to narrow the possible locations of $p$ down to two. Refer to Figures 16.1b,16.1c. Those two locations are reflections of each other over the line through $t_{1}$ and $t_{2}$. In this situation our localization algorithm uses the parity trick. It calculates the distance between the two towers and judging by the parity of this number decides which of the two possible locations is the correct position of $p$.

We also show that $\lfloor 2 n / 3\rfloor$ towers are sometimes necessary. The comb polygon (refer to Fig. 16.4a) from the original art gallery problem can be used to show a lower bound. No point in the comb can look into two different comb spikes. Thus we need at least two towers per spike to localize all of the points in its interior. In addition we need to know the parity trick. Or, alternatively, we need to know $\mathrm{P}$, its exact location and orientation. We show in Theorem 16.3 that without any additional information (such as the parity trick or the complete knowledge about $\mathrm{P}$ including its partition) it is not possible to localize an agent in a simple $n$-gon (where $n=3 k+q$, for integer $k \geqslant 1$ and $q=0,1$ or 2 ) with less than $n-q$ towers.

\subsubsection{Summary of Results}

We present a tower-positioning algorithm that computes a set of size at most $\lfloor 2 n / 3\rfloor$ towers, which improves the previous upper bound of $[8 n / 9\rfloor[17]$. We also show that $[2 n / 3\rfloor$ towers are sometimes necessary. Our localization algorithm uses classical trilateration input (i.e. it receives only the coordinates of the towers that can see the agent $p$ together with their distances to $p$, and has no information about the polygon) and is allowed to know the parity trick.

We strengthen the work [44] by lifting the assumption that the polygon partition produces polygons in general position. We reproved Tóth's result and adapted it, so it can be used with respect to our problem. We found and fixed several mistakes in [44]. In addition we gave a counterexample to the conjecture given by Tóth: any simple polygon of $n$ sides can be guarded by $\lfloor n / 3\rfloor 180^{\circ}$-guards that are located exclusively on the boundary of the polygon. Refer to Section 17.5 in Chapter 17 for a counterexample.

The presented results were submitted to the Journal of Computational Geometry: Theory and Applications (CGTA) [7] in June 2017.

In Chapter 16 we give basic definitions and present some properties and observations. Chapter 17 shows some of our modifications of the polygon partition given by Tóth [44] and its adaptation to our problem. In Chapter 18 we present a localization algorithm.

\subsection{OPTIMAL ART GALLERY LOCALIZATION IS NP-HARD}

Although it has been shown by Chvátal that $\lfloor n / 3\rfloor$ guards are always sufficient and sometimes necessary [13], and such a set of guards can be computed easily [20], such solutions are usually far from optimal in terms of minimizing the number of guards for a particular input polygon. For example, convex polygons and polygons with exactly one reflex angle (angle that is strictly bigger than $180^{\circ}$ ) only require one guard. However, some algorithms for guard positioning would still place $\lfloor n / 3\rfloor$ guards in these situations. It is natural, then, to seek a placement of a minimum number of guards that cover a given polygon. Unfortunately, it was shown that determining an optimal set of guards is NP-hard, even for simple polygons [32]. Refer also to the book "Art Gallery Theorems and Algorithms" by 
O'Rourke [37] that presents more detailed study of the topic. An overview of NP-hardness can be found in the book by Garey and Johnson [24].

We show that the problem of determining the minimum number of broadcast towers that can localize a point anywhere in P is NP-hard. Our solution is closely related to the one done by Lee and Lin in [32]. They study computational complexity of art gallery problems and show that determining the minimum number of point guards for an n-edge simple polygon is NP-hard. To prove NP-hardness of their problem Lee and Lin show a reduction from Boolean Three Satisfiability (3SAT),- a known NPcomplete problem. We use a similar reduction to show that our problem is also NP-hard.

Boolean Three Satisfiability (3SAT) problem:

Instance: $\mathrm{A}$ set $\mathrm{U}=\left\{\mathrm{u}_{1}, \mathrm{u}_{2}, \ldots, \mathrm{u}_{\mathrm{n}}\right\}$ of Boolean variables and a collection $\mathrm{C}=\left\{\mathrm{c}_{1}, \mathrm{c}_{2}, \ldots, \mathrm{c}_{\mathrm{m}}\right\}$ of clauses over $\mathrm{U}$ exist such that $c_{i} \in C$ is a disjunction (OR or $V$ ) of precisely three literals (where a literal is either a variable, or the negation of a variable).

Question: Does there exist a truth assignment to the $\mathrm{n}$ variables in $\mathrm{U}$ such that the conjunctive normal form $(\mathrm{CNF}) c_{1} \wedge c_{2} \wedge \ldots \wedge c_{m}$, evaluates to true?

Our problem:

Art Gallery Localization (AGL) Problem:

Instance: We are given a simple polygon $\mathrm{P}$ of size $\mathrm{n}$ and a positive integer $\mathrm{K}$.

Question: Does a valid set T exist with $|\mathrm{T}| \leqslant \mathrm{K}$ ?

We show that 3SAT can be transformed into AGL in polynomial time. In other words, we polynomially transform a given instance of 3SAT into a simple polygonal region that can be trilaterated by $\mathrm{K}$ or fewer towers if and only if the 3SAT instance is satisfiable. Moreover, we prove that the reduction takes polynomial time. We demonstrate that the number of vertices of the constructed polygon is polynomial in the size of the given input instance of 3SAT and that the number of bits in the binary representation of the coordinates of those vertices is bounded by a polynomial in the size of the input.

\subsubsection{Summary of Results}

We showed that the problem of determining the minimum number of broadcast towers that can localize a point anywhere in $\mathrm{P}$ is NP-hard even if the complete information about the polygon and the coordinates of all the towers are available to the point that wants to locate itself in the art gallery. To prove NP-hardness we showed a reduction from a known NP-complete problem 3SAT to our problem. We proved that the reduction takes polynomial time by showing that the number of vertices of the constructed polygon is polynomial in the size of the input and that the number of bits in the binary representation of the coordinates of those vertices is bounded by a polynomial in the size of the input.

The presented results were submitted to the Journal of Computational Geometry: Theory and Applications (CGTA) [8] in June 2017.

In Chapter 21 we give basic definitions and present some properties and observations. In Chapter 22 we present our main results of NP-hardness. Chapter 23 contains conclusions. 
Part I

ESSENTIAL CONSTRAINTS OF EDGE-CONSTRAINED PROXIMITY GRAPHS 


\section{INTRODUCTION}

This research was inspired by topics in geometric compression. In particular, Devillers et al. [16] investigate how to compute the minimum set $S \subseteq E$ of a given plane triangulation $T=(V, E)$, such that $T$ is a constrained Delaunay triangulation (DT) of the graph $(V, S)$. They show that $S$ and $V$ is the only information that needs to be stored. The graph $T$ can be successfully reconstructed from $S$ and $V$. Experiments on real data sets (such as terrain models and meshes) show that the size of $S$ is less than $3.4 \%$ of the total number of edges of $\mathrm{T}$, which yields an effective compression of the triangulation.

Our goal is to broaden this research and investigate geometric compression of other neighbourhood graphs. We study minimum spanning trees, relative neighbourhood graphs, Gabriel graphs and $\beta$ skeletons for $1 \leqslant \beta \leqslant 2$. We give a definition of each of those graphs in the constraint setting (refer to Chapter 3).

Minimum spanning trees (MST) have been studied for over a century and have numerous applications. We study the problem of finding the minimum set $S$ of constraint edges in a given plane forest $F=(V, E)$ such that the edge-constrained MST over the set $V$ of vertices and the set $S$ of constraints contains $F$. If $F$ is a plane tree then the edge-constrained MST over $(V, S)$ is equal to $F$. We give an $\mathrm{O}(n \log n)$-time algorithm that solves this problem.

Gabriel graphs (GG) were introduced by Gabriel and Sokal in [22]. Toussaint introduced the notion of relative neighbourhood graphs (RNG) in his research on pattern recognition [45]. Both graphs were studied extensively.

Jaromczyk and Kowaluk showed that RNG of a set $\mathrm{V}$ of points can be constructed from the Delaunay triangulation of $\mathrm{V}$ in time $\mathrm{O}(\mathrm{n} \alpha(\mathrm{n}, \mathrm{n}))$, where $\alpha(\cdot)$ is the inverse of the Ackerman function [27]. These two authors, together with Yao, improved the running time of their algorithm to linear [28]. They achieved it by applying a static variant of the Union-Find data structure. They also generalized their algorithm to construct the $\beta$-skeleton $\left(\mathrm{G}_{\beta}\right)$ for $1 \leqslant \beta \leqslant 2$ in linear time from the Delaunay triangulation of $V$ under the $L_{p}$-metric, $1<p<\infty$. We provide the definition of $\beta$-skeleton in Chapter 3 . For now, note that the 1-skeleton corresponds to the Gabriel graph and the 2-skeleton corresponds to the relative neighbourhood graph. In this thesis, we use two geometric structures: elimination path and elimination forest, introduced by Jaromczyk and Kowaluk [27].

Neighbourhood graphs are known to form a nested hierarchy, one of the first versions of which was established by Toussaint [45]: for any $1 \leqslant \beta \leqslant 2, M S T \subseteq R N G \subseteq \mathrm{G}_{\beta} \subseteq \mathrm{GG} \subseteq \mathrm{DT}$. We show that the neighbourhood graphs in the constraint setting form the same hierarchy. Moreover, we show that the minimum set of constraints required to reconstruct a given plane graph (as a part of each of those neighbourhood graphs) form an inverse hierarchy.

In Chapter 3, we present notations and definitions. In Chapter 4, we give some observations concerning constrained MST, show worst-case examples and present an $O(n \log n)$-time algorithm that identifies the minimum set $S \subseteq E$ of constraint edges given a plane forest $F=(V, E)$ such that the edge-constrained MST over the set $V$ of vertices and the set $S$ of constraints contains $F$. In Chapter 5 we address the special case of constrained $\beta$-skeletons - constrained Gabriel graph (1-skeleton). Although the algorithm given in Chapter 6 can be successfully applied to constrained Gabriel graphs, the algorithm presented in Chapter 5 is significantly simpler and requires only local information about an edge in question. Chapter 6 presents an algorithm that identifies the minimum set $S$ of edges of a given plane graph $I=(V, E)$ such that $I \subseteq \mathrm{CG}_{\beta}(V, S)$ for $1 \leqslant \beta \leqslant 2$, where $C_{\beta}(V, S)$ is a constrained $\beta$-skeleton on the set $V$ of vertices and set $S$ of constraints. The hierarchy of the constrained neighbourhood graphs together with the hierarchy of the minimum sets of constraints are given in Chapter 7 . 


\section{BASIC DEFINITIONS}

Let $V$ be a set of $n$ points in the plane and $I=(V, E)$ be a plane graph representing the constraints. Each pair of points $u, v \in V$ is associated with a neighbourhood defined by some property $\mathrm{P}(u, v, \mathrm{I})$ depending on the proximity graph under consideration. An edge-constrained neighbourhood graph $G_{P}(I)$ defined by the property $P$ is a graph with a set $V$ of vertices and a set $E_{P}$ of edges such that $\overline{u v} \in E_{P}$ if and only if $\overline{u v} \in E$ or $\overline{u v}$ satisfies $\mathrm{P}(u, v, I)$. At the end of this chapter you can find an example (Figure 3.2) for each family of edge-constrained neighbourhood graphs that we study.

For clarity and to distinguish between different types of input graphs, if I is a forest, we will denote I by $\mathrm{F}=(\mathrm{V}, \mathrm{E})$, to emphasize its properties.

In this thesis, we assume that the points in $V$ are in general position (no three points are collinear and no four points are co-circular).

Two vertices $u$ and $v$ are visible to each other with respect to $E$ provided that $\overline{u v} \in E$ or the line segment $\overline{u v}$ does not intersect the interior of any edge of $E$. For the following definitions, let $I=(V, E)$ be a plane graph.

DEFINITION 3.I (Visibility Graph of I). The visibility graph of $\mathrm{I}$ is the graph $\mathrm{VG}(\mathrm{I})=\left(\mathrm{V}, \mathrm{E}^{\prime}\right)$ such that $\mathrm{E}^{\prime}=\{(\mathrm{u}, v): \mathrm{u}, v \in \mathrm{V}, \mathrm{u}$ and $v$ are visible to each other with respect to $\mathrm{E}\}$. It is a simple and unweighted graph.

In Def. 3.I, we may think of I as of a set of obstacles. The nodes of VG(I) are the vertices of I, and there is an edge between vertices $u$ and $v$ if they can see each other, that is, if the line segment $\overline{u v}$ does not intersect the interior of any obstacle in I. We say that the endpoints of an obstacle edge see each other. Hence, the obstacle edges form a subset of the edges of $\mathrm{VG}(\mathrm{I})$, and thus $\mathrm{I} \subseteq \mathrm{VG}(\mathrm{I})$.

DEFINITION 3.2 (Euclidean Visibility Graph of I). The Euclidean visibility graph EVG(I) is the visibility graph of $\mathrm{I}$, where each edge $\overline{\mathrm{uv}}(\mathrm{u}, v \in \mathrm{V})$ is assigned weight $w(\mathrm{u}, v)$ that is equal to the Euclidean distance between $u$ and $v$.

DEFINITION 3.3 (Constrained Visibility Graph of I). The constrained visibility graph CVG(I) is the visibility graph of I, where each edge of I is assigned weight 0 and every other edge has weight equal to its Euclidean length.

We use the notation MST $(G)$ to refer to a minimum spanning tree of the graph G. We assume that each edge of $\mathrm{G}$ has weight equal to its Euclidean length, unless the edge was specifically assigned the weight 0 by our algorithm. If none of the edges of $G$ are assigned the weight 0 then MST(G) is a Euclidean minimum spanning tree of G.

Definition 3.4 (Constrained Minimum Spanning Tree of F). Given a plane forest $\mathrm{F}=(\mathrm{V}, \mathrm{E})$, the constrained minimum spanning tree $\mathrm{CMST}(\mathrm{F})$ is the minimum spanning tree of $\mathrm{CVG}(\mathrm{F})$. Refer to Fig. 3.2.

We assume that all the distances between any two vertices of $\mathrm{V}$ are distinct, otherwise, any ties can be broken using lexicographic ordering. This assumption implies that there is a unique MST and a unique CMST.

Since each edge of a plane forest $F$ has weight zero in $C V G(F)$, by running Kruskal on CVG(F), we get $\mathrm{F} \subseteq \mathrm{CMST}(\mathrm{F})$. Notice also that if $\mathrm{F}$ is a plane tree, then $\mathrm{F}=\mathrm{CMST}(\mathrm{F})$.

DEFINITION 3.5 (Locally Delaunay criterion). Let $\mathrm{G}$ be a triangulation, $\overline{v_{1} \nu_{2}}$ be an edge in $\mathrm{G}$ (but not an edge of the convex hull of $\mathrm{G})$, and $\triangle\left(v_{1}, v_{2}, v_{3}\right)$ and $\triangle\left(v_{1}, v_{2}, v_{4}\right)$ be the triangles adjacent to $\overline{v_{1} v_{2}}$ in $\mathrm{G}$. We say that $\overline{v_{1} v_{2}}$ is a locally Delaunay edge if the circle through $\left\{v_{1}, v_{2}, v_{3}\right\}$ does not contain $v_{4}$ or equivalently if the circle through $\left\{v_{1}, v_{2}, v_{4}\right\}$ does not contain $v_{3}$. Every edge of the convex hull of $\mathrm{G}$ is also considered to be locally Delaunay [16]. 
DEFINITION 3.6 (Constrained Delaunay Triangulation of I). The constrained Delaunay triangulation $\mathrm{CDT}(\mathrm{I})$ is the unique triangulation of $\mathrm{V}$ such that each edge is either in $\mathrm{E}$ or locally Delaunay. It follows that $\mathrm{I} \subseteq \mathrm{CDT}(\mathrm{I})$.

This definition is equivalent to the classical definition used for example by Chew in [11]: CDT(I) is the unique triangulation of $V$ such that each edge $e$ is either in $E$ or there exists a circle $C$ with the following properties:

1. The endpoints of edge $e$ are on the boundary of $C$.

2. Any vertex of $I$ in the interior of $C$ is not visible to at least one endpoint of $e$.

The equivalence between the two definitions was shown by Lee and Lin [31].

When considering edge weights of CDT(I), we assume that the weight of each edge is equal to the Euclidean distance between the endpoints of this edge.

The relative neighbourhood graph (RNG) was introduced by Toussaint in 1980 as a way of defining a structure from a set of points that would match human perceptions of the shape of the set [45]. An RNG is an undirected graph defined on a set of points in the Euclidean plane by connecting two points $u$ and $v$ by an edge if there does not exist a third point $p$ that is closer to both $u$ and $v$ than they are to each other. Formally, we can define RNG through the concept of a lune. Let $D(x, r)$ denote an open disk centered at $x$ with radius r, i.e., $\mathrm{D}(x, r)=\{y: \operatorname{dist}(x, y)<r\}$. Let $\mathrm{L}_{\mathfrak{u}, v}=\mathrm{D}(u, \operatorname{dist}(u, v)) \cap$ $\mathrm{D}(v, \operatorname{dist}(\mathrm{u}, v)) ; \mathrm{L}_{\mathfrak{u}, v}$ is called a lune.

DEfinition 3.7 (Relative Neighbourhood graph of V). Given a set V of points, the Relative Neighbourhood graph of $\mathrm{V}, \mathrm{RNG}(\mathrm{V})$, is the graph with vertex set $\mathrm{V}$ and the edges of $\mathrm{RNG}(\mathrm{V})$ are defined as follows: $\overline{u v}$ is an edge if and only if $\mathrm{L}_{\mathrm{u}, v} \cap \mathrm{V}=\emptyset$.

DEFinition 3.8 (Constrained Relative Neighbourhood graph of I). The constrained Relative Neighbourhood graph, CRNG(I), is defined as the graph with vertices $\mathrm{V}$ and the set $\mathrm{E}^{\prime}$ of edges such that each edge $\mathrm{e}=\overline{\mathrm{uv}}$ is either in $\mathrm{E}$ or, $\mathrm{u}$ and $v$ are visible to each other and $\mathrm{L}_{\mathfrak{u}, v}$ does not contain points in $\mathrm{V}$ visible from both $\mathrm{u}$ and $v$. It follows that I $\subseteq$ CRNG(I). Refer to Fig. 3.2.

Gabriel graphs were introduced by Gabriel and Sokal in the context of geographic variation analysis [22]. The Gabriel graph of a set $V$ of points in the Euclidean plane expresses the notion of proximity of those points. It is the graph with vertex set $V$ in which any points $u$ and $v$ of $V$ are connected by an edge if $u \neq v$ and the closed disk with $\overline{u v}$ as a diameter contains no other point of $V$.

DEFINITION 3.9 (Locally Gabriel criterion). The edge $\overline{u v}$ of the plane graph $\mathrm{G}=(\mathrm{V}, \mathrm{E})$ is said to be locally Gabriel if the vertices $u$ and $v$ are visible to each other and the circle with $\overline{u v}$ as a diameter does not contain any points in $\mathrm{V}$ which are visible from both $\mathrm{u}$ and $v$. Refer to Fig. 3.I.

DEFINITION 3.10 (Constrained Gabriel graph of I). The constrained Gabriel graph CGG(I) is defined as the graph with vertices $\mathrm{V}$ and the set $\mathrm{E}^{\prime}$ of edges such that each edge is either in $\mathrm{E}$ or locally Gabriel. It follows that $\mathrm{I} \subseteq \mathrm{CGG}(\mathrm{I})$. Refer to Fig. 3.2.

Relative neighbourhood and Gabriel graphs are special cases of a parametrized family of neighbourhood graphs called $\beta$-skeletons (defined by Kirkpatrick and Radke in [30]). The neighbourhood $\mathrm{U}_{\mathrm{u}, v}(\beta)$ is defined for any fixed $\beta(1 \leqslant \beta<\infty)$ as the intersection of two disks (refer to Fig. 7.1):

$$
\begin{aligned}
\mathrm{u}_{\mathfrak{u}, v}(\beta)= & \mathrm{D}\left(\left(1-\frac{\beta}{2}\right) \mathrm{u}+\frac{\beta}{2} v, \frac{\beta}{2} \operatorname{dist}(\mathrm{u}, v)\right) \cap \\
& \mathrm{D}\left(\left(1-\frac{\beta}{2}\right) v+\frac{\beta}{2} \mathrm{u}, \frac{\beta}{2} \operatorname{dist}(\mathrm{u}, v)\right)
\end{aligned}
$$

DEFINITION 3.II ((lune-based) $\beta$-skeleton of V). Given a set $\mathrm{V}$ of

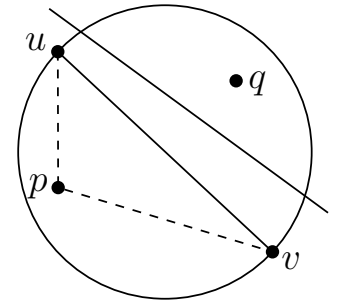

Figure 3.1: Removal of $p$ makes $\overline{u v}$ locally Gabriel.

points in the plane, the (lune-based) $\beta$-skeleton of $\mathrm{V}$, denoted $\mathrm{G}_{\beta}(\mathrm{V})$ is the graph with vertex set $\mathrm{V}$ and the edges of $\mathrm{G}_{\beta}(\mathrm{V})$ are defined as follows: $\overline{\mathrm{uv}}$ is an edge if and only if $\mathrm{U}_{\mathrm{u}, v}(\beta) \cap \mathrm{V}=\emptyset$. 
Notice that $R N G(V)$ is a $\beta$-skeleton of $V$ for $\beta=2$; namely $R N G(V)=G_{2}(V)$. Similarly, $G G(V)=$ $\mathrm{G}_{1}(\mathrm{~V})$.

DEFINITION 3.12 (Constrained $\beta$-skeleton of I). The constrained $\beta$-skeleton of $\mathrm{I}, \mathrm{CG}_{\beta}(\mathrm{I})$ is the graph with vertex set $\mathrm{V}$ and edge set $\mathrm{E}^{\prime}$ defined as follows: $\mathrm{e}=\overline{\mathrm{u} v} \in \mathrm{E}^{\prime}$ if and only if $\mathrm{e} \in \mathrm{E}$ or $\mathrm{u}$ and $v$ are visible to each other and $\mathrm{U}_{u, v}(\beta)$ does not contain points in $\mathrm{V}$ which are visible from both $\mathrm{u}$ and $v$. Refer to Fig. 3.2.

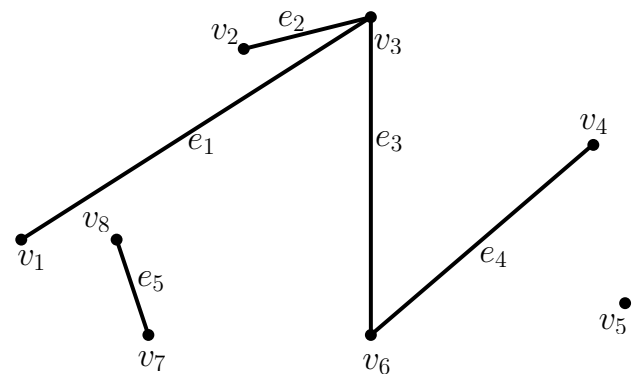

(a) Input graph $F=(V, E)$.

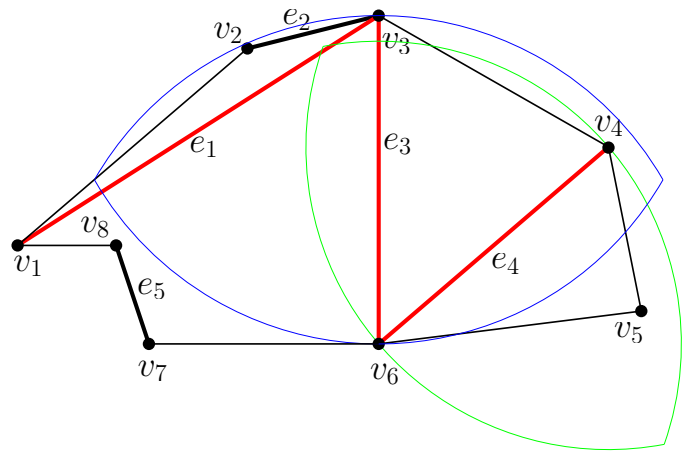

(c) $C R N G(F)=C R N G\left(V,\left\{e_{1}, e_{3}, e_{4}\right\}\right)$. Notice that $e_{3}$ is a constraint because $\mathrm{L}_{v_{3}, v_{6}}$ contains $v_{4}$ and not because it contains $v_{2}$. Since $e_{1}$ is a constraint, $v_{2}$ is not visible to $v_{6}$.

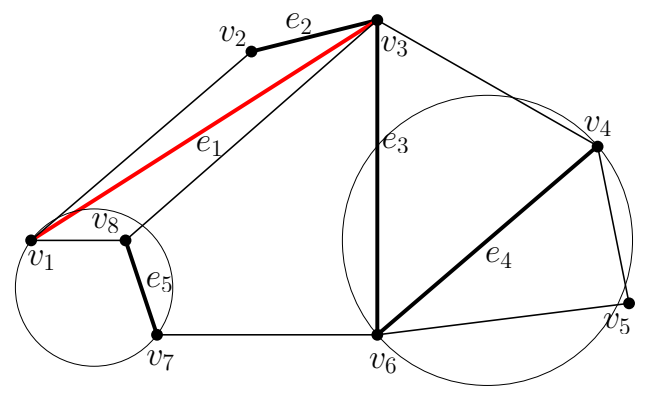

(e) $\operatorname{CGG}(\mathrm{F})=\operatorname{CGG}\left(\mathrm{V},\left\{e_{1}\right\}\right)$.

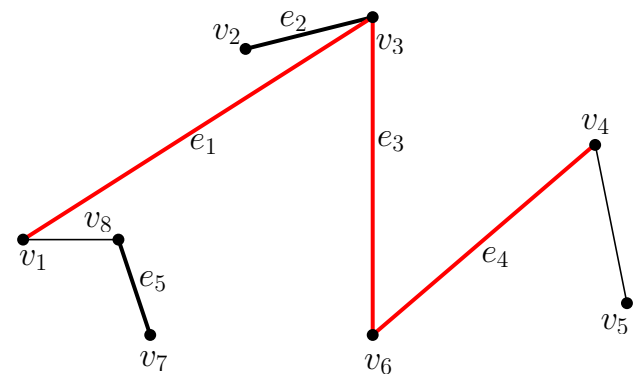

(b) $\operatorname{CMST}(\mathrm{F})=\operatorname{CMST}\left(\mathrm{V},\left\{e_{1}, e_{3}, e_{4}\right\}\right)$.

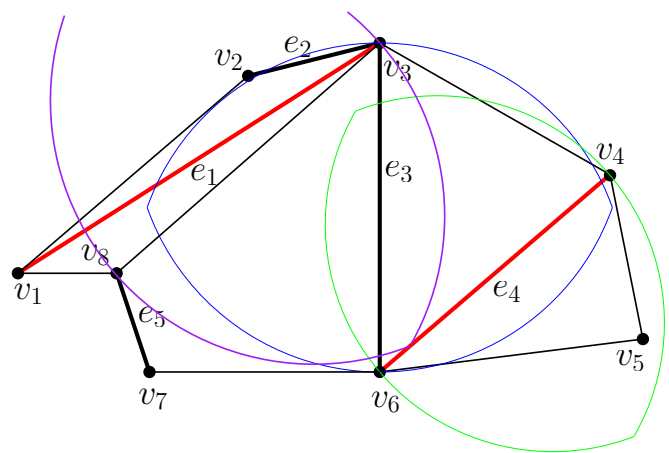

(d) $\mathrm{CG}_{\beta}(\mathrm{F})=\mathrm{CG}_{\beta}\left(\mathrm{V},\left\{e_{1}, e_{4}\right\}\right)$ for $\beta=3 / 2$. Notice that $\overline{v_{3} v_{8}} \in \mathrm{CG}_{\beta}(\mathrm{F})$ by neighbourhood property and despite the fact that $v_{2} \in$ $\mathrm{U}_{v_{3}, v_{8}}(\beta)$ since $v_{2}$ is not visible to $v_{8}$.

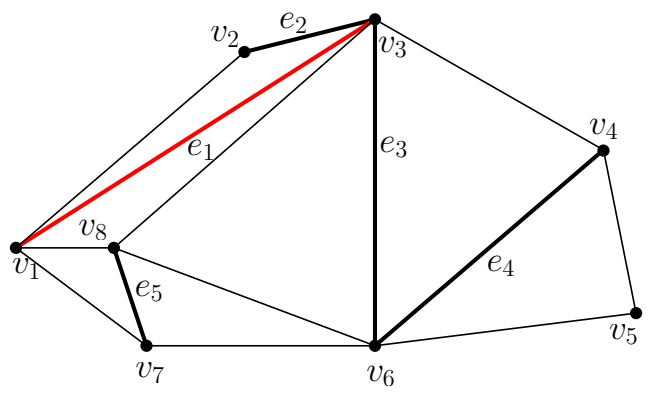

(f) $\operatorname{CDT}(\mathrm{F})=\operatorname{CDT}\left(\mathrm{V},\left\{\mathrm{e}_{1}\right\}\right)$.

Figure 3.2: Example showing relationship between input graph $F$ and its edge-constraint neighbourhood graphs. For every example of a graph, the input edges of $F$ are shown in bold and the minimum set of constraint edges is highlighted in red. 
Problem 1: Let a plane forest $F=(V, E)$ with $|V|=n$ points be given. Find the minimum set $S \subseteq E$ of edges such that $\mathrm{F} \subseteq \mathrm{CMST}(\mathrm{V}, \mathrm{S})$.

In other words, we want to find the smallest subset $S$ of edges of $F$ such that CMST(F) is equal to $\operatorname{CMST}(V, S)$, although the weights of the two trees may be different. Recall, that $\mathrm{CMST}(\mathrm{F})=$ $\operatorname{CMST}(\mathrm{V}, \mathrm{E})$ is the minimum spanning tree of the weighted graph $\mathrm{CVG}(\mathrm{V}, \mathrm{E})$ where each edge of $E$ is assigned weight 0 , and every other edge is assigned a weight equal to its Euclidean length. Notice, that if $\mathrm{F}$ is a tree then $\mathrm{F}=\mathrm{CMST}(\mathrm{F})$. If $\mathrm{F}$ is disconnected then for every edge $e$ of CMST(F) such that $e \notin E$ there exists a cut in $\mathrm{CVG}(\mathrm{V}, \mathrm{E})$ such that $e$ belongs to the cut and its weight is the smallest among other edges that cross the cut (this is true for the same cut in $\operatorname{EVG}(V, E)$ ).

Let us begin by considering an example. We are given a tree $F=\left(\left\{v_{1}, v_{2}, v_{3}\right\},\left\{\overline{v_{1} v_{2}}, \overline{v_{2} v_{3}}\right\}\right)$ (refer to Fig. 4.1a). Figure 4.Ib shows $\operatorname{CDT}(\mathrm{F})$. Observe that $\mathrm{CDT}(\mathrm{F})=\mathrm{DT}\left(\left\{v_{1}, v_{2}, v_{3}\right\}\right)$. In other words, $\operatorname{CDT}(\mathrm{F})=\operatorname{CDT}\left(\left\{v_{1}, v_{2}, v_{3}\right\}, \emptyset\right)$ and thus no constraints are required to construct $\operatorname{CDT}(\mathrm{F})$. However, this is not the case with $\mathrm{CMST}(\mathrm{F})$. Obviously $\mathrm{MST}(\mathrm{CDT}(\mathrm{F})) \neq \mathrm{CMST}(\mathrm{F})$ (refer to Fig. 4.IC), because $\mathrm{F} \nsubseteq \operatorname{MST}(\mathrm{CDT}(\mathrm{F}))$. We need to identify the minimum set $\mathrm{S} \subseteq \mathrm{F}$ of edges such that $\mathrm{F}=\mathrm{CMST}(\mathrm{V}, \mathrm{S})$. In this example $S=\left\{\overline{v_{1} v_{2}}, \overline{v_{2} v_{3}}\right\}$.

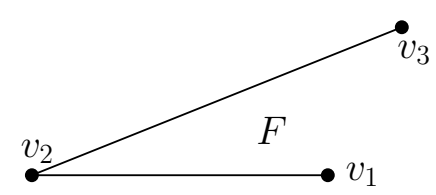

(a)

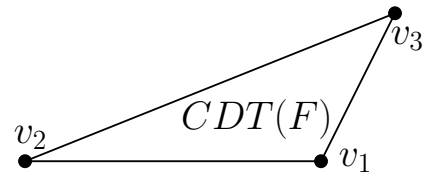

(b)

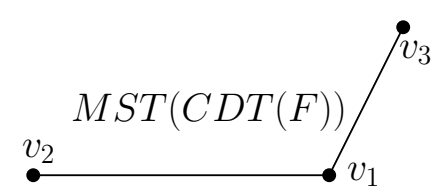

(c)

Figure 4.1: Example showing relationship between input graph, its CDT and MST(CDT). (a) Input graph F. (b) No constraints are required to construct $\operatorname{CDT}(\mathrm{F})$. (c) $\mathrm{F} \neq \mathrm{MST}(\mathrm{CDT}(\mathrm{F}))$. We need two constraints $S=\left\{\overline{v_{1} v_{2}}, \overline{v_{2} v_{3}}\right\}$.

A first idea is to construct an MST of $\operatorname{EVG}(V, \emptyset)$. Every edge of $F$ that is not part of $\operatorname{MST}(E V G(V, \emptyset))$ should be forced to appear in CMST(F). If we do this by adding each such edge of $F$ to $S$ (recall that every edge in $S$ has weight 0 ) then, unfortunately, some edges of $F$, that were part of $\operatorname{MST}(\operatorname{EVG}(V, \emptyset))$, will no longer be part of the MST of the updated graph. A second approach is to start with $\operatorname{MST}(\operatorname{EVG}(V, \emptyset))$ and eliminate every edge that is not part of $F$ and does not connect two disconnected components of $F$. Each such edge $e \in \operatorname{MST}(E V G(V, \emptyset))$ creates a cycle $c_{e}$ in $C M S T(F) \cup\{e\}$ and we have that $c_{e} \subseteq E V G(V, \emptyset)$. If $e$ becomes the heaviest edge of $c_{e}$ then it will no longer be part of the MST. Thus, we add to $S$ every edge of $c_{e} \cap E$ that is heavier than $e$. Although this approach gives us a set $S$ such that $F \subseteq C M S T(V, S)$, the set $S$ of edges with weight 0 may not be minimal. Consider the example of Fig. 4.2. We are given a tree $F=\left(\left\{v_{1}, v_{2}, v_{3}, v_{4}\right\},\left\{\overline{v_{1} v_{2}}, \overline{v_{2} v_{3}}, \overline{v_{3} v_{4}}\right\}\right)$ (refer to Fig. 4.2a). Every edge on the path from $v_{1}$ to $v_{4}$ in $\mathrm{F}$ is heavier than $\overline{v_{1} v_{4}}$ - an edge of $\operatorname{MST}(\operatorname{EVG}(V, \emptyset)) \backslash F$. In order to eliminate $\overline{v_{1} v_{4}}$ from the MST we assign the weight 0 to all the edges of the path $c_{\overline{v_{1} v_{4}}} \backslash \overline{v_{1} v_{4}}$, i.e. $S=\left\{\overline{v_{1} v_{2}}, \overline{v_{2} v_{3}}, \overline{v_{3} v_{4}}\right\}$. However, it is sufficient to assign weight 0 only to the edge $\overline{v_{2} v_{3}}$. In this case, $\operatorname{CMST}\left(\mathrm{V},\left\{\overline{v_{2} v_{3}}\right\}\right)=\mathrm{F}$.

As we will see later this second approach is correct when applied to the MST of a different graph. Instead of considering edges of $\operatorname{MST}(\operatorname{EVG}(\mathrm{V}, \emptyset))$ we apply our idea to $\operatorname{MST}(\mathrm{EVG}(\mathrm{F}))$. Notice that EVG $(F)$ does not have edges that intersect edges of $F$, and thus we will not encounter cases similar to the example of Fig. 4.2. Now it may look like we will be missing important information by considering only a subset of $\operatorname{VG}(\mathrm{V}, \emptyset)$. Can we guarantee that $\operatorname{CMST}(\mathrm{V}, \mathrm{S})$ will not contain edges that intersect 


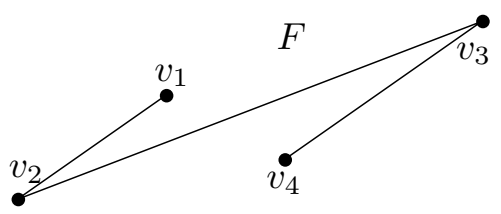

(a)

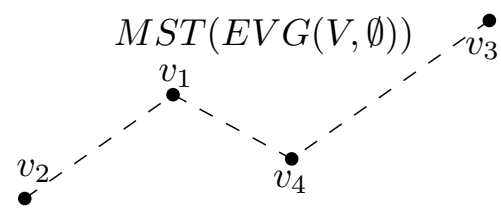

(b)

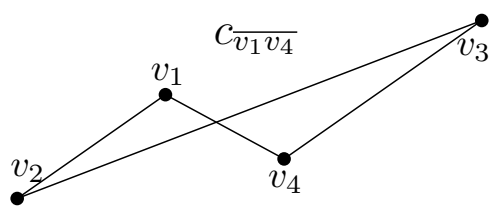

(c)

Figure 4.2: Counterexample to the optimality of $S=\left\{\overline{v_{1} v_{2}}, \overline{v_{2} v_{3}}, \overline{v_{3} v_{4}}\right\}$. The set $S^{\prime}=\left\{\overline{v_{2} v_{3}}\right\}$ is optimal. (a) Input graph $F=(V, E)$. (b) MST of $V$. (c) Cycle $c_{\overline{v_{1} v_{4}}}$ of the graph $F \cup\left\{\overline{v_{1} v_{4}}\right\}$. Every edge on the path from $v_{1}$ to $v_{4}$ in $\mathrm{F}$ is heavier than $\overline{v_{1} v_{4}}$.

edges of $F \backslash S$ ? To answer this question, we prove the following statement: $C M S T(V, S) \subseteq V G(F)$ (Lemma 4.2). The basic algorithm for constructing $S$ is given below. We prove its optimality by showing minimality of S (Lemma 4.4). Later, we present an efficient implementation of this algorithm.

By $\mathrm{CDT}^{\circ}(\mathrm{F})$ we denote $\mathrm{CDT}(\mathrm{F})$ where each edge of $\mathrm{F}$ is assigned weight 0 .

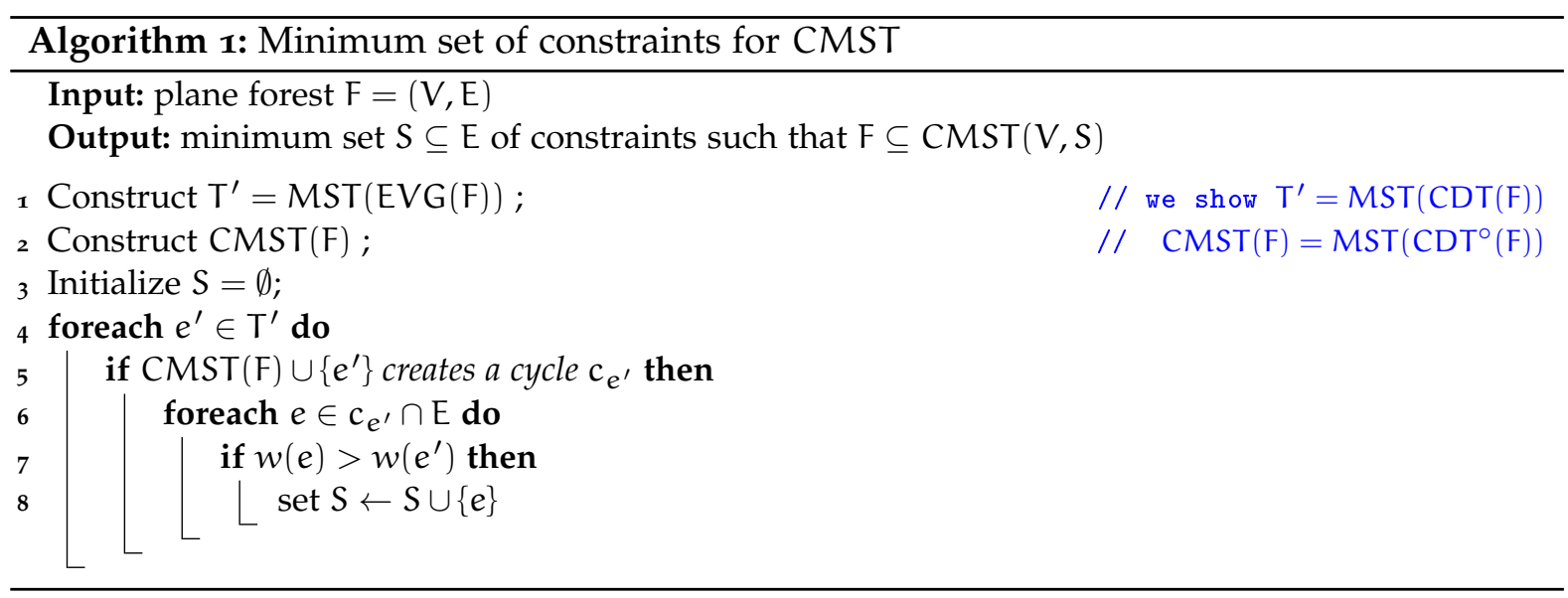

We show the correctness of Algorithm I by proving Lemmas $4.2-4.5$. We start by observing an interesting property of the edges of $F$ that were not added to $S$ during the execution of the algorithm.

PROPERTY 4.1. Let $\mathrm{S}$ be the output of Algorithm I on the input plane forest $\mathrm{F}=(\mathrm{V}, \mathrm{E})$. Let $\mathrm{T}^{\prime}=\mathrm{MST}(\mathrm{EVG}(\mathrm{F}))$. If $e=\overline{u v} \in \mathrm{F}$ and $e \notin \mathrm{S}$ then $e \in \mathrm{T}^{\prime}$.

Proof. Assume to the contrary that $e \notin T^{\prime}$. If we add $e$ to $T^{\prime}$ we create a cycle $c_{e}$. Notice that $e$ is the longest edge among the edges of $c_{e}$. It is given that $e \in \mathrm{F}$ and thus $e \in \mathrm{CMST}(\mathrm{F})$ by Def. 3.4. Let $\mathrm{U}$, $\mathrm{V} \backslash \mathrm{U}$ be a cut that is formed if we delete $e$ from $\mathrm{CMST}(\mathrm{F})$, such that $u \in \mathrm{U}, v \in \mathrm{V} \backslash \mathrm{U}$ (refer to Fig. 4.3). There is a path in $T^{\prime}$ from $u$ to $v$ that does not contain $e$. This path is $c_{e} \backslash\{e\}$. There exists an edge $e^{\prime} \in T^{\prime}$ such that $e^{\prime} \in c_{e}$ and $e^{\prime}$ belongs to the cut $\mathrm{U}, \mathrm{V} \backslash \mathrm{U}$. Notice that $e^{\prime} \notin \operatorname{CMST}(\mathrm{F})$, otherwise $\mathrm{CMST}(\mathrm{F})$ has a cycle. Since $e$ is the longest edge of the cycle $c_{e}$ then $|e|>\left|e^{\prime}\right|$. Because $e^{\prime} \in T^{\prime}$ (and $\left.e^{\prime} \notin \mathrm{CMST}(\mathrm{F})\right)$, Algorithm 1 will consider $e^{\prime}$ in steps $4-8$. Since $e^{\prime}$ belongs to the cut $\mathrm{U}, \mathrm{V} \backslash \mathrm{U}$ in $\operatorname{CMST}(\mathrm{F})$, the cycle $\mathrm{c}_{e^{\prime}}$ in CMST $(\mathrm{F}) \cup\left\{e^{\prime}\right\}$ is formed. Notice that $e \in \mathrm{c}_{e^{\prime}}$ because $e$ and $e^{\prime}$ belong to the cut $U, V \backslash U$. Since $|e|>\left|e^{\prime}\right|$, the edge $e$ is added to $S$. This is a contradiction to $e \notin S$, thus $e \in T^{\prime}$.

LEMMA 4.2. Let $\mathrm{S}$ be the output of Algorithm I on the input plane forest $\mathrm{F}=(\mathrm{V}, \mathrm{E})$. We have $\mathrm{CMST}(\mathrm{V}, \mathrm{S}) \subseteq$ VG(F).

Proof. Let $e^{*}=\overline{\mathrm{ab}}$ be an arbitrary edge of $\operatorname{CMST}(\mathrm{V}, \mathrm{S})$. Assume to the contrary that $e^{*} \notin \mathrm{VG}(\mathrm{F})$ (and hence $e^{*} \notin \mathrm{F}$ and $e^{*} \notin S$ ). Thus there exists an edge of $\mathrm{F}$ that intersects $e^{*}$. Notice, that this edge cannot be in $S$. 


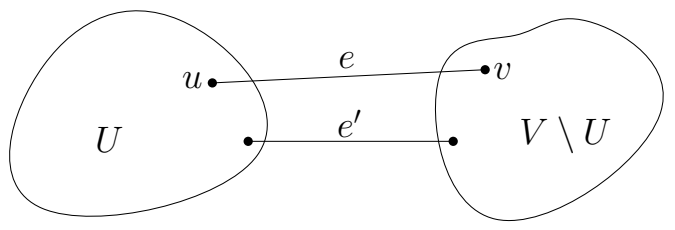

Figure 4.3: The cut $\mathrm{U}, \mathrm{V} \backslash \mathrm{U}$ in $\mathrm{CMST}(\mathrm{F})$ s.t. $\mathrm{u} \in \mathrm{U}$, $v \in \mathrm{V} \backslash \mathrm{U}$.

Let $k(1 \leqslant k \leqslant n)$ be the number of edges of $F$ that intersect $e^{*}$. Let $e_{i}=\overline{c_{i} d_{i}} \in F$ be the edge that intersects $e^{*}$ at point $x_{i}$, where $i(0 \leqslant i<k)$ represents an ordering of edges $e_{i}$ according to the length $\left|\overline{\mathrm{a} x_{i}}\right|$. In other words, the intersection point between $e^{*}$ and $e_{0}$ is the closest to a among other edges of $F$ that intersect $e^{*}$. Refer to Fig. 4.4.

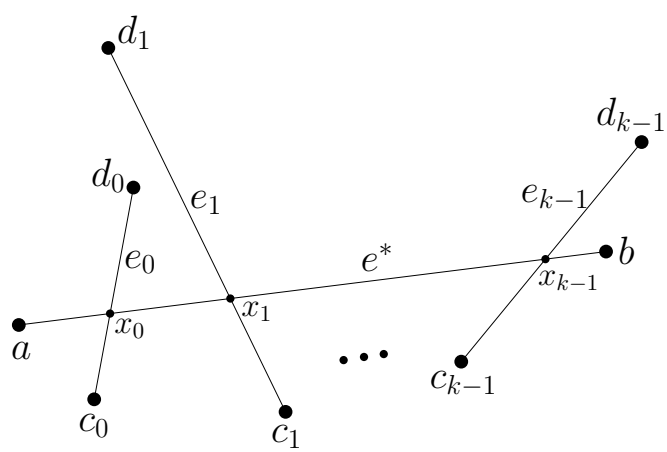

Figure 4.4: Intersection between $e^{*} \in \operatorname{CMST}(\mathrm{V}, \mathrm{S})$ and $k$ edges of $F$. Notice that the points $x_{0}, \ldots, x_{k-1}$ do not belong to $V$.

We prove this lemma in three steps. First we derive some properties of $e_{i}$. Then we show that both endpoints of $e^{*}$ are outside the disk with $e_{i}$ as a diameter for every $0 \leqslant i<k$. We finalize the proof by showing that $e^{*} \notin \mathrm{CGG}(\mathrm{V}, \mathrm{S})$ and thus by Lemma 7.2 (establishing that $\mathrm{CMST}(\mathrm{V}, \mathrm{S}) \subseteq \mathrm{CGG}(\mathrm{V}, \mathrm{S})$, refer to Chapter 7) we have $e^{*} \notin \mathrm{CMST}(\mathrm{V}, \mathrm{S})$. This contradicts the definition of $e^{*}$ which leads to the conclusion that $e^{*} \in \mathrm{VG}(\mathrm{F})$ (meaning that the intersection between $e^{*}$ and an edge of $\mathrm{F}$ is not possible).

Step 1: Since $e_{i} \in F$ and $e_{i} \notin S$ then by Property 4.1: $e_{i} \in M S T(E V G(F))$. Let $D_{e_{i}}$ be the disk with $e_{i}$ as a diameter. There does not exist a point of $V$ inside $D_{e_{i}}$ that is visible to both $c_{i}$ and $d_{i}$. Otherwise, if such a point $v$ exists, then the cycle $\left\{\overline{v c_{i}}, e_{i}, \overline{d_{i} v}\right\}$ is part of $V G(F)$ with $e_{i}$ being the longest edge of this cycle. This contradicts $e_{i} \in M S T(E V G(F))$.

Step 2: Let us first consider $e_{0}$ and show that $a \notin D_{e_{0}}$. Refer to Fig. 4.5a.

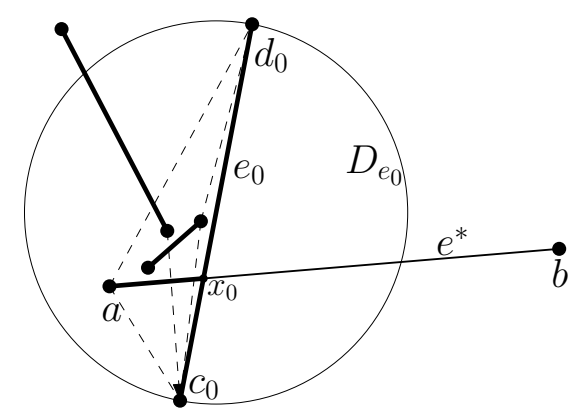

(a) In bold we highlight line segments that cannot be intersected by edges of $F$.

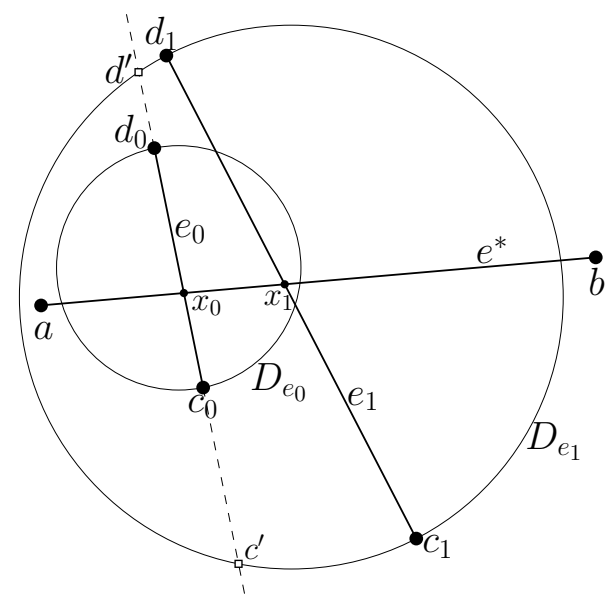

(b) Notice that $\overline{a x_{0}}, \overline{x_{0} x_{1}}, e_{0}$ and $e_{1}$ cannot be intersected by edges of $F$.

Figure 4.5: Both endpoints of $e^{*}$ are outside the disk with $e_{i}$ as a diameter for every $0 \leqslant i<k$. 
Assume to the contrary that $a \in D_{e_{0}}$. We showed in the previous step that there does not exist a point of $V$ inside $D_{e_{0}}$ that is visible to both $c_{0}$ and $d_{0}$. Thus, the point a must be blocked from $c_{0}$ or $d_{0}$ by an edge of $F$. Notice, that edges of $F$ cannot intersect neither line segment $\overline{a x_{0}}$ (refer to the definition of $e_{0}$ ) nor $e_{0}$ (because $F$ is plane and $e_{0} \in F$ ). Therefore, the edge that blocks a from $c_{0}$ (respectively, $\left.d_{0}\right)$ must have one endpoint inside the triangle $\triangle\left(a, c_{0}, x_{0}\right)$ (respectively, $\triangle\left(a, x_{0}, d_{0}\right)$ ). Since both triangles are inside $\mathrm{D}_{e_{0}}$, this endpoint also belongs to $\mathrm{D}_{e_{0}}$ and must not be visible to both $c_{0}$ and $d_{0}$. It must be blocked similarly to a. Since we have a finite number of points in $V$ there will be a point in $D_{e_{0}}$ that will not be blocked from $c_{0}$ and $d_{0}$. This is a contradiction to $a \in D_{e_{0}}$.

Consider the edge $e_{1}$. We want to show that $a \notin D_{e_{1}}$. We proved that $a \notin D_{e_{0}}$. Assume to the contrary that $a \in D_{e_{1}}$ and thus $\angle\left(c_{1}, a, d_{1}\right)>\pi / 2$. Refer to Fig. $4.5 b$. It follows, that $x_{0} \in D_{e_{1}}$. We claim that at least one endpoint of $e_{0}$ belongs to $D_{e_{1}}$. Assume to the contrary that $c_{0}, d_{0} \notin D_{e_{1}}$. Then the edge $e_{0}$ intersects the boundary of $D_{e_{1}}$ in points $c^{\prime}$ and $d^{\prime}$. Since $e_{0}$ and $e_{1}$ do not intersect, by construction the chord $\overline{c^{\prime} d^{\prime}}$ lies between $a$ and $e_{1}$ and therefore $\angle\left(c^{\prime}, a, d^{\prime}\right)>\angle\left(c_{1}, a, d_{1}\right)>\pi / 2$. On the other hand, since $a \notin D_{e_{0}}$, we have $\angle\left(c^{\prime}, a, d^{\prime}\right)<\angle\left(c_{0}, a, d_{0}\right)<\pi / 2$. We have a contradiction and thus at least one endpoint of $e_{0}$ belongs to $D_{e_{1}}$. Without loss of generality assume that $c_{0} \in D_{e_{1}}$. We proved in step 1 that there are no points of $V$ inside $D_{e_{1}}$ that are visible to both $c_{1}$ and $d_{1}$. Therefore, the point $c_{0}$ must be blocked from $c_{1}$ or $d_{1}$ by an edge of $F$. Notice that $\overline{x_{0} x_{1}}, e_{0}$ and $e_{1}$ cannot be intersected by edges of $F$. It follows that one endpoint of the blocking edge must be inside quadrilateral $\left\{c_{0}, c_{1}, x_{1}, x_{0}\right\}$ or triangle $\triangle\left(x^{\prime}, x_{1}, d_{1}\right)$, where $x^{\prime}$ is the intersection point between $\overline{c_{0} d_{1}}$ and $e^{*}$. Both polygons belong to $D_{e_{1}}$ and thus the endpoint of the blocking edge also belongs to $D_{e_{1}}$. This endpoint cannot be visible to both $c_{1}$ and $d_{1}$ and thus must be blocked by another edge of $F$. Since the number of points in $V$ is finite there will be a point in $D_{e_{1}}$ that will be visible to both $c_{1}$ and $d_{1}$. This is a contradiction and thus $\mathrm{a} \notin \mathrm{D}_{e_{1}}$.

In a similar way we can show that $a \notin D_{e_{i}}$ for every $e_{i}, 0 \leqslant i<k$. Symmetrically, it is also true that $\mathrm{b} \notin \mathrm{D}_{e_{i}}$ for $0 \leqslant i<k$. Thus we have shown that for every edge $e \in \mathrm{F}$ that intersects $e^{*}$, both endpoints of $e^{*}$ are outside $\mathrm{D}_{\mathrm{e}}$.

Step 3: In the previous step we showed that $a, b \notin D_{e_{i}}$ for $0 \leqslant i<k$. Thus $e^{*}$ intersects the boundary of $D_{e_{i}}$ at the points $a^{\prime}$ and $b^{\prime}$. Refer to Fig. 4.6.

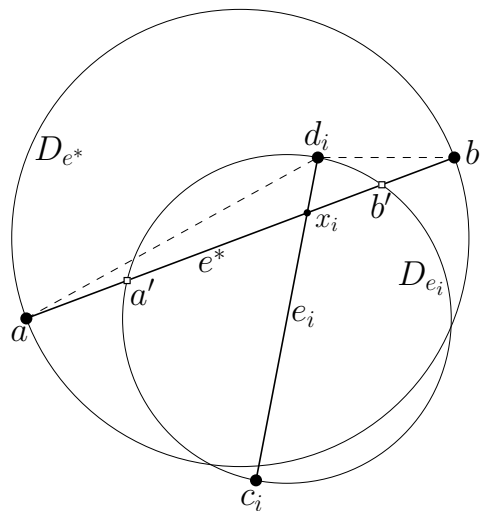

Figure 4.6: Intersection between $e^{*} \in \operatorname{CMST}(\mathrm{V}, \mathrm{S})$ and an edge of $F$.

Consider the chord $\overline{a^{\prime} b^{\prime}}$ of $D_{e_{i}}$. Since $e_{i}$ and $\overline{a^{\prime} b^{\prime}}$ intersect, then $\angle\left(a^{\prime}, d_{i}, b^{\prime}\right) \geqslant \pi / 2$ or $\angle\left(b^{\prime}, c_{i}, a^{\prime}\right) \geqslant$ $\pi / 2$. Without loss of generality assume that $\angle\left(a^{\prime}, d_{i}, b^{\prime}\right) \geqslant \pi / 2$. It follows that $\angle\left(a, d_{i}, b\right)>\pi / 2$ and thus $d_{i} \in D_{e^{*}}$. Consider the graph $V G(V, S)$. We are given that $e^{*} \in \operatorname{CMST}(V, S)$. We prove in Lemma 7.2 (refer to Chapter 7 ) that $\mathrm{CMST}(\mathrm{V}, \mathrm{S}) \subseteq \mathrm{CGG}(\mathrm{V}, \mathrm{S})$. Since $e^{*} \notin \mathrm{S}$ then by Def. 3.9 and 3.10 the disk $D_{e^{*}}$ does not contain points in $V$ which are visible from both $a$ and $b$. Therefore the point $d_{i}$ must be blocked from $a$ or $b$ by an edge of $S$. Notice, that edges of $S$ cannot intersect neither $e^{*}$ (because $e^{*} \in \mathrm{VG}(\mathrm{V}, \mathrm{S})$ ) nor $e_{i}$ (because $S \subseteq E$ and $\mathrm{F}$ is plane). Thus, one endpoint of the edge that blocks $d_{i}$ from $a$ or $b$ must be inside the triangle $\triangle\left(a, b, d_{i}\right)$. This endpoint belongs to $D_{e^{*}}$ (since $\left.\triangle\left(a, b, d_{i}\right) \in D_{e^{*}}\right)$ and therefore must be blocked from $a$ or $b$ by another edge of $S$. We have a finite 
number of points in $V$ and thus there is a point of $V$ in $D_{e^{*}}$ that is not blocked from $a$ and $b$. This is a contradiction to $e^{*} \in \mathrm{CGG}(\mathrm{V}, \mathrm{S})$.

We conclude that an intersection between an edge of $\mathrm{F}$ and an edge of $\mathrm{CMST}(\mathrm{V}, \mathrm{S})$ is not possible. Therefore $e^{*} \in \mathrm{VG}(\mathrm{F})$, meaning $\mathrm{CMST}(\mathrm{V}, \mathrm{S}) \subseteq \mathrm{VG}(\mathrm{F})$.

LEMMA 4.3. Let $\mathrm{S}$ be the output of Algorithm $\mathrm{I}$ on the input plane forest $\mathrm{F}=(\mathrm{V}, \mathrm{E})$. We have $\mathrm{F} \subseteq$ $\operatorname{CMST}(\mathrm{V}, \mathrm{S})$.

Proof. Let $e=\overline{u v}$ be an arbitrary edge of $\mathrm{F}$. We have to show that $e \in \operatorname{CMST}(\mathrm{V}, \mathrm{S})$. There are two cases to consider:

1. $e \in \mathrm{S}$ : then $e \in \operatorname{CMST}(\mathrm{V}, \mathrm{S})$ by Def. 3.4 .

2. $e \notin S$ : then by Property 4.I we have $e \in \operatorname{MST}(E V G(F))$. Notice, that $e \in C M S T(F)$ because $e \in F$. Consider the cut $\mathrm{U}, \mathrm{V} \backslash \mathrm{U}$ in $\mathrm{CMST}(\mathrm{F})$ such that $u \in \mathrm{U}, v \in \mathrm{V} \backslash \mathrm{U}$ (refer to Fig. 4.7).

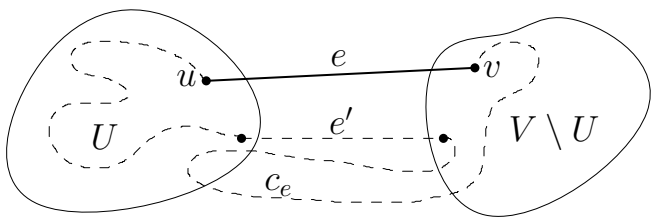

Figure 4.7: The cut $\mathrm{U}, \mathrm{V} \backslash \mathrm{U}$ in $\mathrm{CMST}(\mathrm{F})$ s.t. $\mathrm{u} \in \mathrm{U}$, $v \in \mathrm{V} \backslash \mathrm{U}, e \in \mathrm{F}$. The path $\mathrm{c}_{e} \backslash\{e\}$ from $u$ to $v$ in $\mathrm{CMST}(\mathrm{V}, \mathrm{S})$ is shown in dashed line. By Lemma 4.2 none of the edges of this path intersects $e$. Notice, that $e, e^{\prime} \notin S$ and $e$ is the heaviest edge of the cycle $c_{e}$, thus $w(e)>w\left(e^{\prime}\right)$.

Notice, that none of the edges of $S$ belong to this cut, otherwise CMST(F) has a cycle (because $\mathrm{S} \in \mathrm{F} \subseteq \mathrm{CMST}(\mathrm{F}))$. Assume to the contrary that $e \notin \mathrm{CMST}(\mathrm{V}, \mathrm{S})$. If we add $e$ to $\mathrm{CMST}(\mathrm{V}, \mathrm{S})$ we will create a cycle $c_{e}$, that contains the path $c_{e} \backslash\{e\}$ from $u$ to $v$ in $\operatorname{CMST}(V, S)$. By Lemma 4.2 we have $c_{e} \subseteq \mathrm{VG}(\mathrm{F})$. Therefore, there exist an edge $e^{\prime} \in c_{e}$ that belongs to the cut $\mathrm{U}, \mathrm{V} \backslash \mathrm{U}$ in $\mathrm{VG}(\mathrm{F})$. Notice, that $e$ must be the heaviest edge of the cycle $c_{e}$, therefore, since $e^{\prime} \notin S$ then $|e|>\left|e^{\prime}\right|$ and thus $w(e)>w\left(e^{\prime}\right)$. Two cases are possible:

a) $e^{\prime} \in \operatorname{MST}(\operatorname{EVG}(\mathrm{F})):$ Algorithm 1 considers $e^{\prime}$ in step 4. Since $e$ and $e^{\prime}$ belong to the same cut $\mathrm{U}, \mathrm{V} \backslash \mathrm{U}$ a cycle in $\mathrm{CMST}(\mathrm{F}) \cup\left\{e^{\prime}\right\}$ is formed. This cycle contains e. Because $|e|>\left|e^{\prime}\right|$ the edge $e$ is added to $S$. This contradicts to $e \notin S$.

b) $e^{\prime} \notin \operatorname{MST}(\operatorname{EVG}(\mathrm{F}))$ : there exist a cycle $c_{e^{\prime}}$ in the graph $\operatorname{MST}(\operatorname{EVG}(\mathrm{F})) \cup\left\{e^{\prime}\right\}$ such that $e^{\prime}$ is the heaviest edge of this cycle. Consider the cut $\mathrm{U}^{\prime}, \mathrm{V} \backslash \mathrm{U}^{\prime}$ in $\operatorname{CMST}(\mathrm{V}, \mathrm{S})$ such that one endpoint of $e^{\prime}$ is in $U^{\prime}$ and another is in $V \backslash U^{\prime}$ (refer to Fig. 4.8). There exist an edge $\widehat{e}$ of $c_{e^{\prime}}$ that belongs to the cut $U^{\prime}, V \backslash U^{\prime}$ in $V G(F)$. Since $e^{\prime} \notin S$ we can delete it from $\operatorname{CMST}(V, S)$. Notice that $\operatorname{CMST}(V, S) \backslash\left\{e^{\prime}\right\} \cup\{\widehat{e}\}$ is a tree that contains all the edges of $S$. Moreover, since $\left|e^{\prime}\right|>|\widehat{e}|$ the weight of $\operatorname{CMST}(V, S) \backslash\left\{e^{\prime}\right\} \cup\{\widehat{e}\}$ is smaller than the weight of $\operatorname{CMST}(V, S)$, which is a contradiction.

We showed a contradiction to the fact that that $e \notin \mathrm{CMST}(\mathrm{V}, \mathrm{S})$ and thus $\mathrm{F} \subseteq \mathrm{CMST}(\mathrm{V}, \mathrm{S})$.

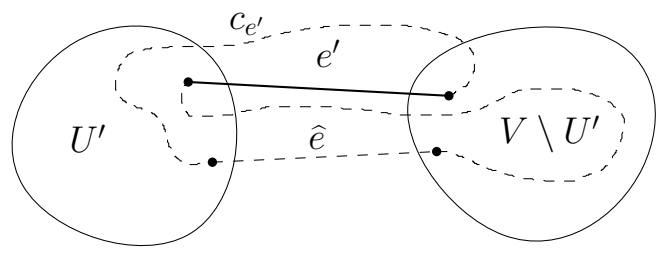

Figure 4.8: The cut $\mathrm{U}^{\prime}, \mathrm{V} \backslash \mathrm{U}^{\prime}$ in $\operatorname{CMST}(\mathrm{V}, \mathrm{S})$ s.t. $e^{\prime} \in$ $\operatorname{CMST}(\mathrm{V}, \mathrm{S})$ and $e^{\prime}$ belongs to this cut. Recall that $e^{\prime} \notin S$. The cycle $c_{e^{\prime}}$ in MST(EVG(F)) $U$ $\left\{e^{\prime}\right\}$ is shown in dashed line. By Lemma 4.2 none of the edges of this cycle is intersected by $e^{\prime}$. Notice, that $e^{\prime}$ is the heaviest edge of $c_{e^{\prime}}$.

LEMMA 4.4. Let $\mathrm{S}$ be the output of Algorithm I on the input plane forest $\mathrm{F}=(\mathrm{V}, \mathrm{E})$. The set $\mathrm{S}$ is minimal and minimum. 
Proof. Let us first prove that the set $S$ is minimal. Assume to the contrary that $S$ is not minimal. Thus there is an edge $e \in S$ such that $\mathrm{F} \subseteq \mathrm{CMST}(\mathrm{V}, \mathrm{S} \backslash\{\mathrm{e}\})$. We have to disprove that $\mathrm{F}$ is a subgraph of $\mathrm{CMST}(\mathrm{V}, \mathrm{S} \backslash\{\boldsymbol{e}\})$. Let $\mathrm{T}$ be $\mathrm{CMST}(\mathrm{V}, \mathrm{S} \backslash\{\boldsymbol{e}\})$. Since $e \in \mathrm{S}$, the edge $e$ was added to $\mathrm{S}$ by Algorithm 1 . Thus, there exists an edge $e^{\prime} \in \operatorname{MST}(\mathrm{EVG}(\mathrm{F}))$ such that $e^{\prime} \notin \mathrm{F}$ (and thus $e^{\prime} \notin \mathrm{CMST}(\mathrm{F})$ ); and there is a cycle $c_{e^{\prime}}$ in $\operatorname{CMST}(F) \cup\left\{e^{\prime}\right\}$ such that $e \in c_{e^{\prime}}$ and $|e|>\left|e^{\prime}\right|$. Notice, that $T^{*}=T \backslash\{e\} \cup\left\{e^{\prime}\right\}$ is a tree. Moreover, $\mathrm{T}^{*}$ is a subgraph of $\mathrm{CVG}(\mathrm{V}, \mathrm{S} \backslash\{e\})$ whose weight is smaller than the weight of $\mathrm{T}$. This is a contradiction to $\mathrm{T}$ being $\mathrm{CMST}(\mathrm{V}, \mathrm{S} \backslash\{\mathrm{e}\})$.

Let us now prove that the set $S$ is minimum. Assume to the contrary that there is another set $S^{*} \subseteq \mathrm{E}$ of constraints, such that $\mathrm{F} \subseteq \operatorname{CMST}\left(\mathrm{V}, \mathrm{S}^{*}\right)$ and $\left|\mathrm{S}^{*}\right|<|\mathrm{S}|$. We have to show a contradiction to $\mathrm{F} \subseteq \mathrm{CMST}\left(\mathrm{V}, \mathrm{S}^{*}\right)$.

We proved that $S$ is minimal, thus $S \backslash S^{*} \neq \emptyset$. Let $e=\overline{u v}$ be an edge of $S \backslash S^{*}$. Notice, that $e \in F$. Consider the cut $\mathrm{U}, \mathrm{V} \backslash \mathrm{U}$ in $\mathrm{CMST}(\mathrm{F})$ such that $\mathrm{u} \in \mathrm{U}, v \in \mathrm{V} \backslash \mathrm{U}$ (refer to Fig. 4.3). Algorithm $\mathrm{I}$ added $e$ to $S$, thus there exists an edge $e^{\prime} \in \operatorname{MST}(\operatorname{EVG}(\mathrm{F}))$ such that $e^{\prime} \notin \mathrm{E}, e$ and $e^{\prime}$ belong to the cycle $c_{e^{\prime}}$ in $\mathrm{CMST}(\mathrm{F}) \cup\left\{e^{\prime}\right\}$ and $|e|>\left|e^{\prime}\right|$. Notice, that $e^{\prime}$ belongs to the cut $\mathrm{U}, \mathrm{V} \backslash \mathrm{U}$ in $\mathrm{VG}(\mathrm{F})$. Moreover, $e^{\prime} \in \mathrm{CVG}\left(\mathrm{V}, \mathrm{S}^{*}\right)$ because $S^{*} \subseteq \mathrm{E}$ and $e^{\prime}$ does not intersect edges of $\mathrm{F}$. Since $e$ is not a constraint in $\operatorname{CMST}\left(\mathrm{V}, \mathrm{S}^{*}\right)$, then its weight is not $\mathrm{O}$ but equal to the Euclidean distance between its endpoints. Thus, the inequality $w(e)>w\left(e^{\prime}\right)$ is true for $\operatorname{CVG}\left(V, S^{*}\right)$.

The edge $e^{\prime}$ is not intersected by any edge of $F$ (because $e^{\prime} \in \operatorname{VG}(F)$ ) and thus the graph $G=$ $\mathrm{CMST}\left(\mathrm{V}, \mathrm{S}^{*}\right) \backslash\{\mathrm{e}\} \cup\left\{\mathrm{e}^{\prime}\right\}$ is a plane tree and $w(\mathrm{G})<w\left(\mathrm{CMST}\left(\mathrm{V}, \mathrm{S}^{*}\right)\right)$. Thus $\mathrm{G}$ is the true $\mathrm{CMST}\left(\mathrm{V}, \mathrm{S}^{*}\right)$. However $\mathrm{F} \nsubseteq \mathrm{G}$ because $e \notin \mathrm{G}$. This is a contradiction.

Lemmas 4.3 and 4.4 show the correctness of Algorithm 1. However, we said nothing about our strategy of finding cycles in the graph. With a naive approach step $4-8$ of the algorithm could be quadratic in $n$. Also, the size of the visibility graph of $F$ can be quadratic in the size of $V$, leading to the complexity of step 1 of the algorithm equal to $\mathrm{O}\left(n^{2} \log n\right)$. Our first step to improve the running time is to reduce the size of the graph we construct MST for. Lemma 4.5 shows that MST(EVG(F)) $\subseteq$ CDT(F). The same lemma can be used to show that CMST $(\mathrm{F})$ can be constructed from CDT(F). Notice that $\mathrm{CDT}(\mathrm{F})$ has size $\mathrm{O}(\mathrm{n})$. The running time of steps 1 and 2 then becomes $\mathrm{O}(n \log n)$. Moreover, if $\mathrm{F}$ is a plane tree then the construction of $\mathrm{CDT}(\mathrm{F})$ can be performed in $\mathrm{O}(\mathrm{n})$ time [12].

LEMMA 4.5. Given a plane forest $\mathrm{F}=(\mathrm{V}, \mathrm{E})$ we have $\mathrm{MST}(\mathrm{EVG}(\mathrm{F})) \subseteq \mathrm{CDT}(\mathrm{F})$. Notice that the edges of both graphs EVG(F) and CDT(F) are assigned weights equal to the Euclidean distance between the endpoints of corresponding edges. Similarly, MST $(\mathrm{CVG}(\mathrm{F})) \subseteq \mathrm{CDT}(\mathrm{F})$.

Proof. Let $e=\overline{u v}$ be an arbitrary edge of MST(EVG(F)). If $e \in F$ then by Def. 3.6, $e \in \operatorname{CDT}(\mathrm{F})$. Assume that $e \notin \mathrm{F}$. Consider the graph CDT(F). We consider the circle with the line segment $\overline{u v}$ as a diameter Suppose there is a point $p$ inside this circle, that is visible to $u$ and $v$. Refer to Fig. 3.1. Then we have $|\overline{p u}|<|\overline{u v}|$ and $|\overline{p v}|<|\overline{u v}|$ (by $|\overline{p u}|$ we denote the length of the line segment $\overline{p u}$ ). Consider the cycle $u \rightarrow p \rightarrow v \rightarrow u$. We know that the heaviest edge on a cycle does not belong to any MST. Hence, $e \notin M S T(E V G(F))$ because $e$ is the heaviest edge of that cycle. This is a contradiction to the assumption that there is a point $p$ inside the circle. Therefore, there is a circle having $u$ and $v$ on its boundary that does not have any vertex of $\mathrm{F}$ visible to $\mathrm{u}$ and $v$ in its interior. By Def. 3.5 the pair of vertices $u$ and $v$ form a locally Delaunay edge, thus $e \in \operatorname{CDT}(F)$.

In a similar we can show that $\mathrm{MST}(\mathrm{CVG}(\mathrm{F})) \subseteq \mathrm{CDT}(\mathrm{F})$.

We use the Link/Cut Tree of Sleator and Tarjan [43] to develop an efficient solution for the fourth step (lines $4-8$ ) of Algorithm 1. The complexity of the algorithm becomes $\mathrm{O}(n \log n)$.

\subsection{AN EFFICIENT IMPLEMENTATION OF ALGORITHM 1}

In this section we develop an efficient solution for the step 4-8 of Algorithm 1. We use Link/Cut Tree type of data structure invented by Sleator and Tarjan in 1981 [43]. It is also referred in literature 
as a Dynamic Trees data structure. This data structure maintains a collection of rooted vertex-disjoint trees and supports two main operations: link (that combines two trees into one by adding an edge) and cut (that divides one tree into two by deleting an edge). Each operation requires $\mathrm{O}(\log n)$ time. We consider the version of dynamic trees problem that maintains a forest of trees, each of whose edges has a real-valued cost. We are also interested in a fast search for an edge that has the maximal cost among edges on a tree path between a pair of given vertices. We can implement this with the help of original mincost (vertex $v$ ) operation (that returns the vertex $w$ closest to the root of $v$ such that the edge between $w$ and its parent has minimum cost among edges on the tree path from $v$ to the root of $v$ ). However, for that to work in our case we have to negate weights of our problem. Alternatively, we can create maxcost(vertex $v$ ) operation, by implementing it similarly to mincost(vertex $v$ ). Then we can use unaltered weights. Below is a brief list of operations we use. Refer to [43] for a detailed description and implementation.

- parent(vertex $v$ ): Returns the parent of $v$, or null if $v$ is a root and thus has no parent.

- $\operatorname{root}(\operatorname{vertex} v)$ : Returns the root of the tree containing $v$.

- $\operatorname{cost}(\operatorname{vertex} v)$ : Returns the cost of the edge $(v, \operatorname{parent}(v))$. This operation assumes that $v$ is not a tree root.

- $\operatorname{maxcost}(\operatorname{vertex} v)$ : Returns the vertex $u$ closest to $\operatorname{root}(v)$ such that the edge $(u$, parent $(u))$ has maximum cost among edges on the tree path from $v$ to $\operatorname{root}(v)$. This operation assumes that $v$ is not a tree root.

- $\operatorname{link}(\operatorname{vertex} v, u$, real $x)$ : Combines the trees containing $v$ and $u$ by adding the edge $(v, u)$ of cost $x$, making $u$ the parent of $v$. This operation assumes that $v$ and $u$ are in different trees and $v$ is a tree root.

- $\operatorname{cut}(\operatorname{vertex} v)$ : Divides the tree containing vertex $v$ into two trees by deleting the edge $(v$, par$\operatorname{ent}(v))$; returns the cost of this edge. This operation assumes that $v$ is not a tree root.

- update edge(vertex $v$, real $x)$ : Adds $x$ to the cost of the edge $(v, \operatorname{parent}(v))$.

- lca(vertex $v, u)$ : Returns the lowest common ancestor of $v$ and $u$, or returns null if such an ancestor does not exist.

The data structure uses $O(n)$ space and each of the above operations takes $\mathrm{O}(\log n)$ time. Algorithm 2 finds the minimum set $S \subseteq E$ for a given forest $F=(V, E)$ such that $\mathrm{F} \subseteq \mathrm{CMST}(\mathrm{V}, \mathrm{S})$. Algorithm's complexity is $\mathrm{O}(n \log n)$.

If $\mathrm{F} \subseteq \mathrm{MST}(\mathrm{V})$ then $\mathrm{F} \subseteq \mathrm{CMST}(\mathrm{V}, \emptyset)$. In other words we do not require constraints at all to obtain Constrained MST that will contain F. It is interesting to consider the opposite problem. How big can the set of constraints be? Figure 4.9 shows the worst-case example, where the set $S$ of constraints contains all the edges of $F$, thus $|S|=n-1$.

THEOREM 4.6. Given a plane forest $\mathrm{F}=(\mathrm{V}, \mathrm{E})$, Algorithm 2 constructs the minimum set $\mathrm{S} \subseteq \mathrm{E}$ of constraints such that $\mathrm{F} \subseteq$ $\mathrm{CMST}(\mathrm{V}, \mathrm{S})$. The running time of Algorithm 2 is $\mathrm{O}(\mathrm{n} \log \mathrm{n})$, where $\mathrm{n}=|\mathrm{V}|$.

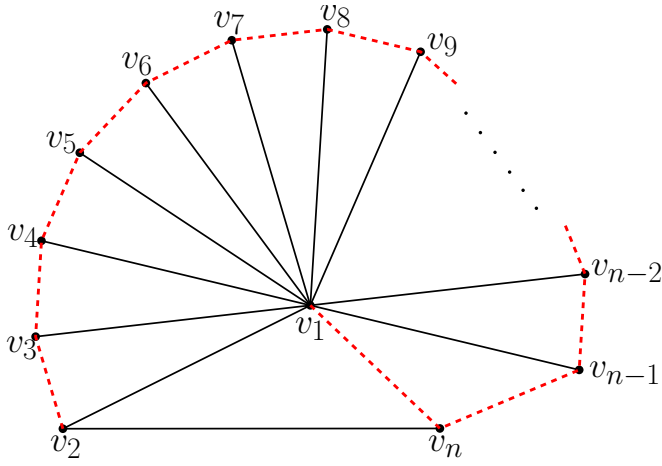

Figure 4.9: Worst case example showing $n-1$ constraints. The input tree $F$ is drawn using solid lines. The MST of the set $\left\{v_{1}, v_{2}, \ldots v_{n}\right\}$ is dashed. 


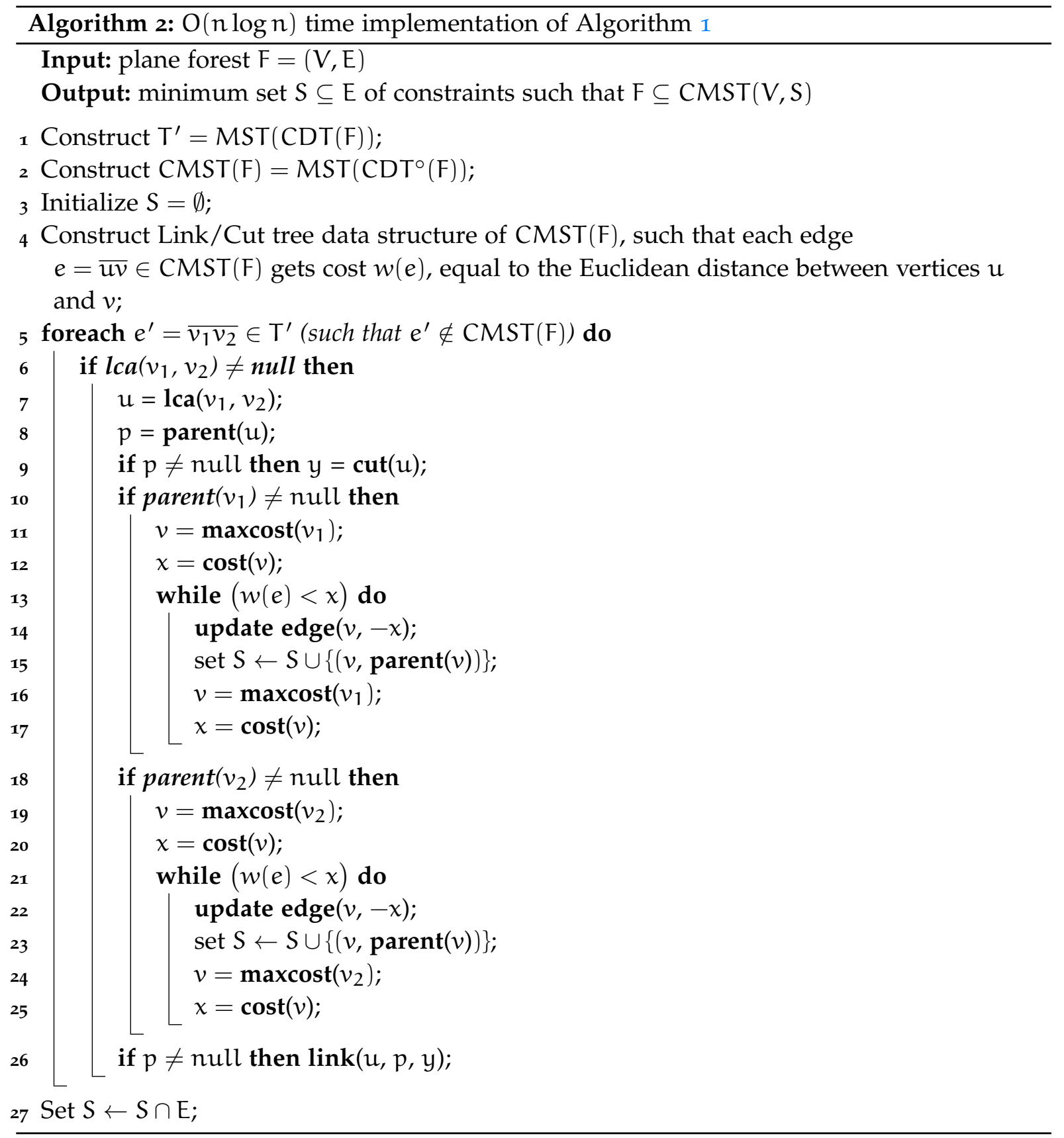


Problem 2: We are given a plane graph $I=(V, E)$ of $|V|=n$ points. Find the minimum set $S \subseteq E$ of edges such that $I \subseteq C G G(V, S)$. In other words, we are interested in the minimum set $S \subseteq E$ of edges such that $\mathrm{CGG}(\mathrm{V}, \mathrm{S})=\mathrm{CGG}(\mathrm{I})$.

Algorithm 5 given in Chapter 6 can be successfully applied to CGGs. However, the algorithm presented in this chapter is significantly simpler and requires only local information about an edge in question. We can decide whether or not the edge $e \in I$ should be in $S$ by considering at most two triangles adjacent to $e$ in $\mathrm{CDT}(\mathrm{I})$. This can be done in constant time. We exploit the fact that $S$, constructed of all non locally Gabriel edges of I, is necessary and sufficient. Consider the following lemma.

LEMMA 5.1. Let $\mathrm{I}=(\mathrm{V}, \mathrm{E})$ be a plane graph and let $\mathrm{S}$ be the set of all the edges of $\mathrm{E}$ that are not locally Gabriel. Then $\mathrm{S}$ is the minimum set of constraints such that $\mathrm{I} \subseteq \mathrm{CGG}(\mathrm{V}, \mathrm{S})$.

Proof. Let us first show that $\mathrm{I} \subseteq \mathrm{CGG}(\mathrm{V}, \mathrm{S})$. Indeed, $\mathrm{S} \subseteq \mathrm{I}$ so we can add edges to $\mathrm{S}$ to obtain $\mathrm{E}$. Thus, we add $E \backslash S$, which, by definition, consists of locally Gabriel edges only. Therefore, there is no edge in I that does not belong to $C G G(V, S)$. So, $S$ is a sufficient set of constraints.

Let us show that $S$ is necessary. Let $S^{\prime}$ be a subset of edges in I such that $I \subseteq C G G\left(V, S^{\prime}\right)$ and $S \backslash S^{\prime} \neq \emptyset$. Let $e=(u, v)$ be an edge in $S \backslash S^{\prime}$. By definition, the edge $e$ is not locally Gabriel and thus there exists a point $p \in V$ such that the circle with $e$ as a diameter contains $p$. Since $I \subseteq C G G\left(V, S^{\prime}\right)$, then in particular $e \in \operatorname{CGG}(\{u, v, p\}, \emptyset)$ which is false since $e$ is not locally Gabriel. Hence such an edge cannot exist and thus $S$ is minimum.

The above lemma gives us a tool for constructing S. We need to find a proper graph that is both relatively small in size and keeps required information about each edge of I easily accessible. As you may have already guessed, CDT(I) is a good candidate. We show in Lemma 7.2 that CGG(I) $\subseteq$ CDT(I). Thus if an edge is locally Gabriel in CGG(I) then it is locally Delaunay in CDT(I). Applied to our problem, it means that if an edge of $I$ is not a constraint in CGG(I) then it is not a constraint in CDT(I). The opposite however is not true. Refer to Fig. 5.I for an example of an edge that is locally Delaunay, but not locally Gabriel.

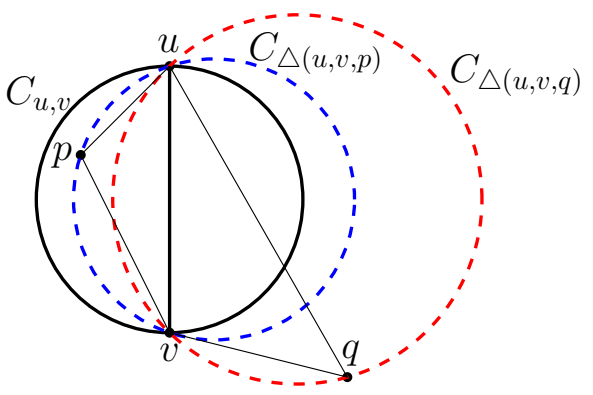

Figure 5.1: The edge $\overline{u v}$ is locally Delaunay, but not locally Gabriel. If $\overline{u v} \in I$ then $\overline{u v} \notin$ $\mathrm{S}_{\mathrm{CDT}(\mathrm{I})}$ and $\overline{\mathrm{uv}} \in \mathrm{S}_{\mathrm{CGG(I)}}$. If $\overline{\mathrm{u} v} \notin \mathrm{I}$ then $\overline{u v} \notin \mathrm{CGG}(\mathrm{I})$.

We prove in Lemma $7 \cdot 3$ that $S_{\mathrm{CGG}(\mathrm{I})} \supseteq S_{\mathrm{CDT}(\mathrm{I})}$, where $S_{\mathrm{G}}$ denotes the minimum set of constraints of $G$, such that $I \subseteq G$. This means that if some edge of $I$ is a constraint in $\operatorname{CDT}\left(V, S_{C D T(I)}\right)$ then the same edge is also a constraint in $\mathrm{CGG}\left(\mathrm{V}, \mathrm{S}_{\mathrm{CGG}(\mathrm{I})}\right)$. Notice, that $\mathrm{CDT}\left(\mathrm{V}, \mathrm{S}_{\mathrm{CDT}(\mathrm{I})}\right)=\mathrm{CDT}(\mathrm{I})$ and $\mathrm{CGG}\left(\mathrm{V}, \mathrm{S}_{\mathrm{CGG}(\mathrm{I})}\right)=\mathrm{CGG}(\mathrm{I})$. Thus, if $\mathrm{S}_{\mathrm{CDT}(\mathrm{I})}$ is known then we can speed up our algorithm by initializing $S$ with $S_{\mathrm{CDT}(\mathrm{I})}$.

The only question that is left unanswered is how can we tell in constant time if an edge of I is locally Gabriel or not by observing CDT(I). The following lemma answers this question. 
LEMMA 5.2. Let $\mathrm{I}=(\mathrm{V}, \mathrm{E})$ be a plane graph and let $\overline{\mathrm{uv}}$ be an edge of $\mathrm{I}$ such that $\overline{\mathrm{uv}}$ is not a constraint edge of $\mathrm{CDT}(\mathrm{I})$. Let $\triangle(\mathrm{u}, v, \mathrm{p})$ be a triangle in $\mathrm{CDT}(\mathrm{I})$, and let $\mathrm{C}_{\mathfrak{u}, v}$ be the circle with diameter $\overline{u v}$. If $\mathrm{p} \notin \mathrm{C}_{\mathfrak{u}, v}$ then there is no point $\mathrm{x}$ of $\mathrm{V}$ on the same side of $\overline{\mathrm{uv}}$ as $\mathrm{p}$ such that $\mathrm{x} \in \mathrm{C}_{\mathrm{u}, v}$ and $\mathrm{x}$ is visible to both $\mathrm{u}$ and $v$ in CGG(I). Refer to Fig. 5.2.

Proof. Assume to the contrary, that such a point $x$ exists. Since $x$ is visible to both $u$ and $v$ in $C G G(I)$, then since $S_{\mathrm{CGG}(\mathrm{I})} \supseteq S_{\mathrm{CDT}(\mathrm{I})}$ (refer to Lemma 7.3) there are no constraints in CDT(I) intersected by the line segments $\overline{u x}$ and $\overline{v x}$. Refer to Fig. 5.2. Since $p \notin C_{u, v}$, the angle $\angle v p u$ is acute. Because $x \in$

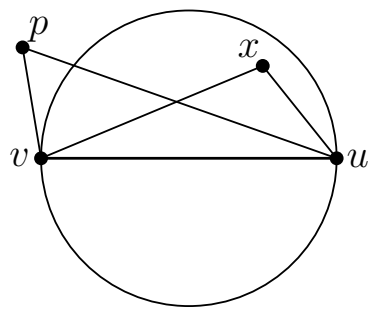

Figure 5.2: Proof of Lemma 5.2. $\mathrm{C}_{\mathrm{u}, v}$, the angle $\angle v x u$ is obtuse. Consider the circle $\mathrm{C}^{\prime}$ circumscribing the triangle $\triangle(u, v, p)$. Both angles $\angle v p u$ and $\angle v x u$ are supported by the same chord $\overline{u v}$, and since $x$ and $p$ are on the same side of $\overline{u v}$ and $\angle v p u<\angle v x u$, then $x$ is inside $C^{\prime}$. The edge $\overline{u p}$ is not locally Delaunay and since it belongs to CDT(I), then $\overline{u p}$ must be a constraint. The line segment $\overline{v x}$ and the constraint $\overline{u p}$ intersect, which is a contradiction to the existence of the point $x$.

We are ready to present an algorithm for constructing $S$.

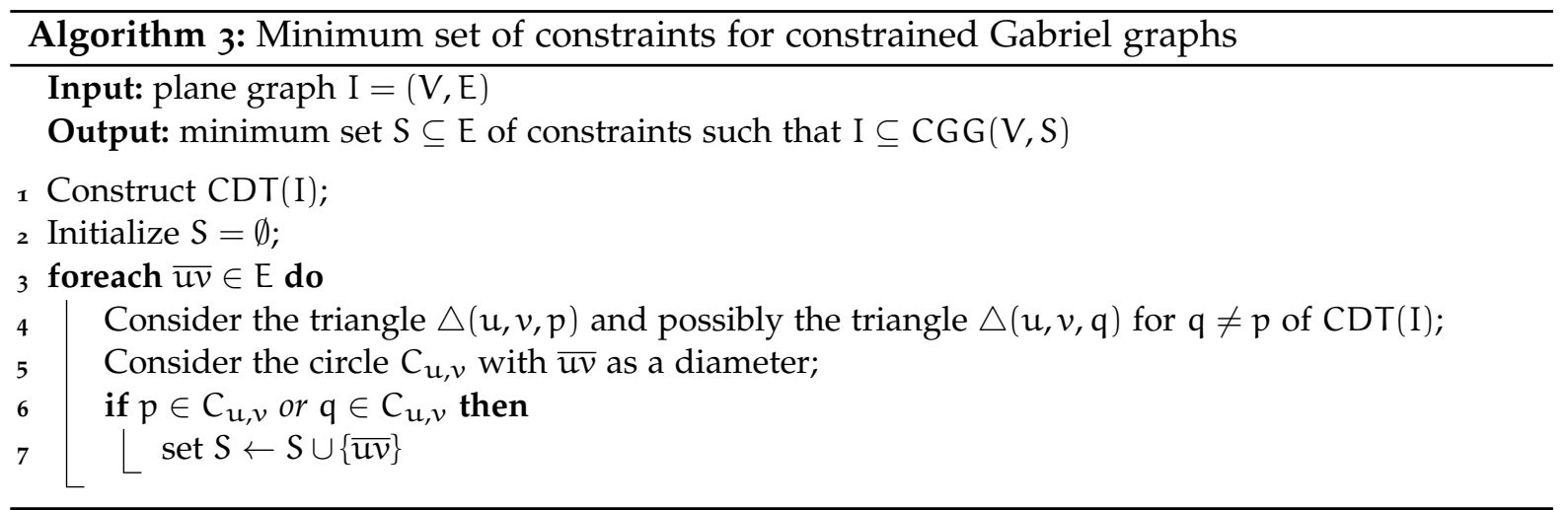

Notice that $\mathrm{CGG}(\mathrm{V}, \mathrm{S}) \subseteq \mathrm{CDT}(\mathrm{I})$, because $\mathrm{CGG}(\mathrm{V}, \mathrm{S})=\mathrm{CGG}(\mathrm{I}) \subseteq \mathrm{CDT}(\mathrm{I})$. Lemma 5.2 justifies step $3-7$ of the algorithm. Notice, that the set $S$ constructed by Algorithm 3, is the set of all the edges of I that are not locally Gabriel. Thus, by Lemma 5.I, $S$ is the minimum set of constraints such that $\mathrm{I} \subseteq \mathrm{CGG}(\mathrm{V}, \mathrm{S})$. This concludes the proof of correctness of Algorithm 3 .

The running time of step 1 of Algorithm 3 is $O(n \log n)$ in the worst case. The step $3-7$ of the algorithm takes $\mathrm{O}(\mathrm{n})$ time. If the input graph $\mathrm{I}$ is a simple polygon, a triangulation or a tree then the running time of the first step of the algorithm can be reduced to $\mathrm{O}(\mathrm{n})$, leading to a linear total running time for this algorithm.

THEOREM 5.3. Given a plane graph $\mathrm{I}=(\mathrm{V}, \mathrm{E})$, Algorithm 3 constructs the minimum set $\mathrm{S} \subseteq \mathrm{E}$ of constraints such that $\mathrm{I} \subseteq \mathrm{CGG}(\mathrm{V}, \mathrm{S})$. The running time of Algorithm 3 is $\mathrm{O}(\mathrm{n} \log \mathrm{n})$, where $\mathrm{n}=|\mathrm{V}|$. 
Problem 3: We are given a plane graph $I=(V, E)$ of $|V|=n$ points and $1 \leqslant \beta \leqslant 2$. Find the minimum set $S \subseteq E$ of edges such that $I \subseteq \mathrm{CG}_{\beta}(\mathrm{V}, \mathrm{S})$. In other words, we are interested in the minimum $S$ such that $\mathrm{CG}_{\beta}(\mathrm{V}, \mathrm{S})=\mathrm{CG}_{\beta}(\mathrm{I})$.

For the constrained Gabriel graph, the problem can be solved in a simpler way. We show that $S$, constructed of all non locally Gabriel edges of I, is necessary and sufficient. We can decide in constant time whether or not the edge $e \in I$ is locally Gabriel by considering at most two triangles adjacent to $e$ in $\operatorname{CDT}(\mathrm{I})$. Refer to Chapter 5.

Let $u, v$ and $p$ be a triple of vertices of $V$. Recall the definition of $U_{u, v}(\beta)$; see Chapter 3 and Fig. 6.1a. If $p \in U_{u, v}(\beta)$ and $p$ is visible to both $u$ and $v$, then we say that the vertex $p$ eliminates line segment $\overline{u v}$. We prove in Lemma 7.2 (refer to Chapter 7) that $C R N G(I)=\mathrm{CG}_{\beta=2}(\mathrm{I}) \subseteq \mathrm{CG}_{1 \leqslant \beta \leqslant 2}(\mathrm{I}) \subseteq \mathrm{CG}_{\beta=1}(\mathrm{I})=$ $\mathrm{CGG}(\mathrm{I}) \subseteq \mathrm{CDT}(\mathrm{I})$. The following lemmas further explain a relationship between $\mathrm{CG}_{\beta}(\mathrm{I})$ and $\mathrm{CDT}(\mathrm{I})$.

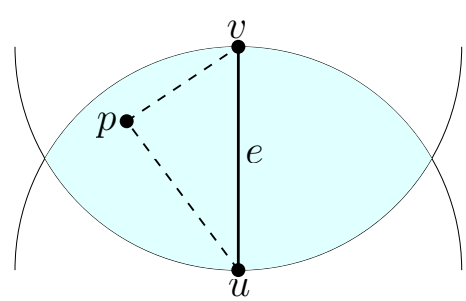

(a)

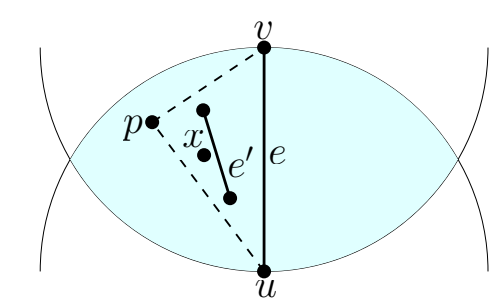

(b)

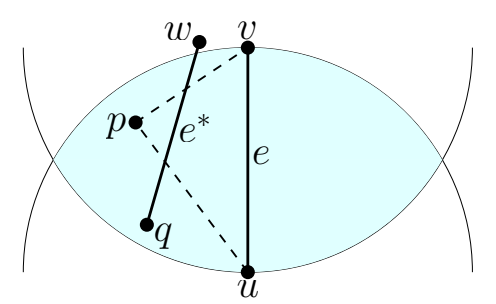

(c)

Figure 6.1: The neighbourhood $U_{u, v}(\beta=2)$ is highlighted in cyan. (a) The vertex $p \in V$ eliminates $\overline{u v}$. (b) If $p$ is the closest vertex to $\overline{u v} \in \operatorname{CDT}(\mathrm{I})$ that eliminates $\overline{u v}$, then $\triangle(u, v, p) \cap V=\emptyset$. (c) If $p$ is the closest vertex to $\overline{u v} \in \operatorname{CDT}(\mathrm{I})$ that eliminates $\overline{u v}$, then $p \in U_{e^{*}}(\beta)$ for every edge of $e^{*} \in \mathrm{CDT}(\mathrm{I})$ that lies between $p$ and $\overline{u v}$.

LEMMA 6.1. Given a plane graph $\mathrm{I}=(\mathrm{V}, \mathrm{E})$ and $1 \leqslant \beta \leqslant 2$. Let $\mathrm{p} \in \mathrm{V}$ be the closest vertex to the edge $\overline{u v} \in \operatorname{CDT}(\mathrm{I})$ that eliminates $\overline{u v}$. Then $\triangle(\mathfrak{u}, v, p) \cap \mathrm{V}=\emptyset$. Refer to Fig. 6.I.

Proof. Assume to the contrary that there exist a vertex $x \in \mathrm{V}$ such that the triangle $\triangle(u, v, p)$ contains $x$. The vertex $x$ does not eliminate $\overline{u v}$, otherwise it will be a contradiction to $p$ being the closest vertex to $\overline{u v}$ that eliminates $\overline{u v}$. Thus, there exist an edge $e^{\prime} \in I$ that blocks $x$ from $u$ or $v$, i.e. $e^{\prime}$ intersects line segment $\overline{u x}$ or $\overline{v x}$. Refer to Fig. 6.Ib. Notice, that $e^{\prime}$ does not intersect neither $\overline{u p}$ nor $\overline{v p}$, otherwise $p$ is not visible to both $u$ and $v$, which contradicts to the fact that $p$ eliminates $\overline{u v}$. Therefore, both endpoints of $e^{\prime}$ are inside $\triangle(u, v, p)$. Let us look at the endpoint of $e^{\prime}$ that is closest to $\overline{u v}$ (let us call it $\left.x^{\prime}\right)$. It is clear that $x^{\prime}$ is closer to $\overline{u v}$ than $x$. Similarly to $x, x^{\prime}$ must be blocked by an edge of I from either $u$ or $v$. Since we have a finite number of vertices, there will be a vertex inside $\triangle(u, v, p)$ visible to both $u$ and $v$ and closer to $\overline{u v}$ than $p$. This is a contradiction.

The above lemma implies that if there exists an edge $e^{*} \in \operatorname{CDT}(\mathrm{I})$ that lies between $p$ and $\overline{u v}$, i.e. $e^{*}$ intersects the interior of $\triangle(u, v, p)$, then $e^{*}$ intersects both line segments $\overline{u p}$ and $\overline{v p}$. Refer to Fig. 6.Ic. Notice, that $e^{*}$ cannot intersect $\overline{u v}$, since $e^{*}, \overline{u v} \in \operatorname{CDT}(\mathrm{I})$.

LEMMA 6.2. Given a plane graph $\mathrm{I}=(\mathrm{V}, \mathrm{E})$ and $1 \leqslant \beta \leqslant 2$. Let $\mathrm{p} \in \mathrm{V}$ be the closest vertex to the edge $\overline{u v} \in \operatorname{CDT}(\mathrm{I})$ that eliminates $\overline{\mathrm{uv}}$. Let $e^{*}=\overline{\mathrm{q} w}$ be an edge of $\operatorname{CDT}(\mathrm{I})$ that intersects $\triangle(\mathrm{u}, v, \mathrm{p})$. Then $\mathrm{p} \in \mathrm{U}_{\mathrm{q}, w}(\beta)$. Ref. to Fig. 6.1c. 
Proof. According to Lemma 6.1, the edge $e^{*}$ intersects $\overline{u p}$ and $\overline{v p}$. Refer to Fig. 6.1c. Notice, that $e^{*} \notin \mathrm{I}$, otherwise $p$ is not visible to $u$ and $v$. The endpoints of $e^{*}$ may or may not belong to $u_{u, v}(\beta)$. Notice, that if an endpoint of $e^{*}$ belongs to $U_{u, v}(\beta)$ then it must be either further away from $\bar{u} v$ than $p$ or it must be blocked by some edge of I from being visible to $u$ or $v$. Observe, that $\angle(q, p, w)>\angle(u, p, v)$. Thus, since $p \in U_{u, v}(\beta)$ then $p \in U_{q, w}(\beta)$.

To solve our main problem we will use two geometric structures: elimination path and elimination forest, introduced by Jaromczyk and Kowaluk in [27]. The elimination path for a vertex $p$ (starting from an adjacent triangle $\triangle(p, u, v) \in \operatorname{CDT}(I))$ is an ordered list of edges, such that $p \in U_{e}(\beta)$ for each edge $e$ of this list. In the work [27] an edge $e$ belongs to the elimination path induced by some point $p$ only if $e$ is eliminated by $p$. In our problem this is not the case. The point $p$ eliminates $e$ if and only if $p \in U_{e}(\beta)$ and $p$ is visible to both endpoints of $e$. We show how to adapt the original elimination forest to our problem later in this chapter.

The elimination path is defined by the following construction (refer to Algorithm 4). Notice, that the construction of the elimination path introduced by Jaromczyk and Kowaluk in [27] does not contain step 4.

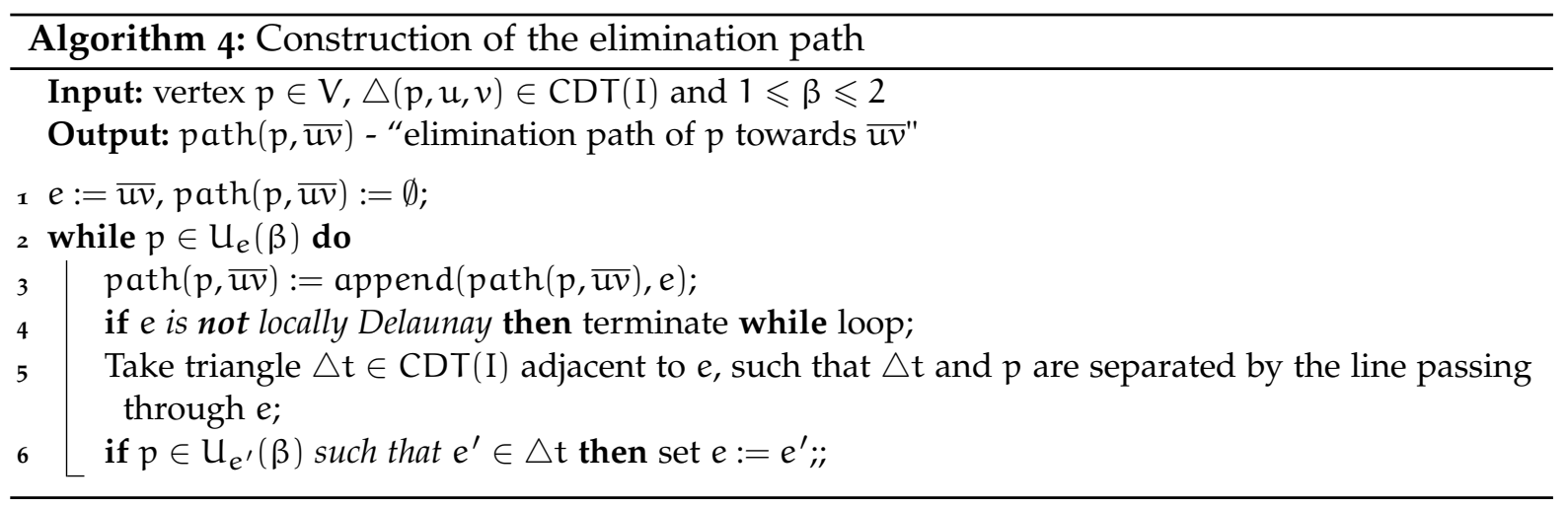

Jaromczyk and Kowaluk show that a vertex cannot belong to the $\beta$-neighbour- hood $\mathrm{U}(\beta)$ of more than two edges for a particular triangle. Thus elimination paths do not split. Moreover, they also show that if two elimination paths have a common edge $e$ and they both reached $e$ via the same triangle, then starting from this edge one of the two paths is completely included in another one. This property is very important - it guarantees that the elimination forest can be constructed in linear time. Refer to [27] and [28] for further details.

Since we are dealing with CDT, the elimination paths defined via the original construction may split at non-locally Delaunay edges. To overcome this problem we terminate the propagation of the elimination path after a non-locally Delaunay edge is encountered and added to the path. Thus, the elimination forest for our problem can also be constructed in linear time. It is shown in Lemma 2.3 in [27] that if two points eliminate a common edge of a triangle in DT (such that both points are external to this triangle) then the two points can eliminate at most one of the remaining edges of this triangle. Similarly, we can show that if two points of $V$ eliminate a common locally Delaunay edge $e$ of an external triangle in CDT then they can eliminate at most one of the remaining edges of the triangle. It is due to the fact that there exists a circle that contains the endpoints of e such that if any vertex $v$ of $V$ is in the interior of the circle then it cannot be "seen" from at least one of the endpoints of $e$. It means that the point $v$ does not eliminate $e,-$ the elimination path of $v$ terminated at non-locally Delaunay edge that obstructed visibility between $v$ and one of the endpoints of $e$.

Lemmas 6.1 and 6.2 show that no important information will be lost as a result of "shorter" elimination paths. Every non-locally Delaunay edge of $\mathrm{CDT}(\mathrm{I})$ is a constraint and thus belongs to I. Edges of I obstruct visibility. Let $p \in V$ be the closest vertex to the edge $\overline{u v} \in \operatorname{CDT}(\mathrm{I})$ that eliminates $\overline{u v}$. Assume to the contrary that $\overline{u v}$ is not on the elimination path from $p$ because the path terminated at 
non-locally Delaunay edge $e^{*}$ before the path could reach $\overline{u v}$. By Lemma 6.I the edge $e^{*}$ intersects both line segments $\overline{p u}$ and $\overline{p v}$. Refer to Fig. 6.Ic. Since $e^{*} \in I$, neither $u$ nor $v$ are visible to the point $p$. This contradicts the fact that $p$ eliminates $\overline{u v}$. Lemma 6.3 further shows that if some edge $e$ of I must be a constraint in $\mathrm{CG}_{\beta}(\mathrm{I})$ then it will belong to at least one elimination path, and in particular, to the path induced by the closest point to $e$ that eliminates $e$.

LEMMA 6.3. Given a plane graph $\mathrm{I}=(\mathrm{V}, \mathrm{E})$ and $1 \leqslant \beta \leqslant 2$. Let $\mathrm{p} \in \mathrm{V}$ be the closest vertex to the edge $\overline{u v} \in \operatorname{CDT}(\mathrm{I})$ that eliminates $\overline{\mathrm{uv}}$. Then $\overline{\mathrm{uv}}$ belongs to the elimination path induced by $\mathrm{p}$.

Proof. Let $\mathrm{E}^{\prime}$ be the set of all the edges of CDT(I) that lie between $p$ and $\overline{u v}$. By Lemma 6.I each of those edges intersects both line segments $\overline{p u}$ and $\overline{p v}$. We proved in Lemma 6.2 that $p \in U_{e^{*}}(\beta)$ for every edge $e^{*} \in E^{\prime}$. Since $p$ eliminates $\overline{u v}$, none of the edges of $E^{\prime}$ belong to I and thus every edge of $E^{\prime}$ is locally Delaunay. Let $e^{\prime}$ be the closest edge to $p$ among all the edges of $E^{\prime}$. It follows that every edge of $E^{\prime}$ belongs to the elimination path induced by $p$ towards edge $e^{\prime}$ : $p a t h\left(p, e^{\prime}\right)$. Therefore, the edge $\overline{u v}$ is also appended to this elimination path at a certain point.

Each elimination path starts with a special node (we call it a leaf) that carries information about the vertex that induced the current elimination path. A node that corresponds to the last edge of a particular elimination path also carries information about the vertex that started this path. The elimination forest is build from bottom (leaves) to top (roots).

The elimination forest (let us call it ElF) gives us a lot of information, but we still do not know how to deal with visibility. The elimination path induced by point $p$ can contain locally Delaunay edges of I that may obstruct visibility between $p$ and other edges that are further on the path. We want to identify edges that not only belong to the elimination path of some vertex $p$ but whose both endpoints are also visible to $p$. Observe, that visibility can only be obstructed by edges of I. Let us contract all the nodes of the ElF that correspond to edges not in I. If a particular path is completely contracted, we delete its corresponding leaf as well. Now the ElF contains only nodes of edges that belong to I together with leaves, that identify elimination paths, that originally had at least one edge of I. The correctness of our approach is supported by the following lemma.

LEMMA 6.4. Given a plane graph $\mathrm{I}=(\mathrm{V}, \mathrm{E}), 1 \leqslant \beta \leqslant 2$ and a contracted ElF of $\mathrm{CDT}(\mathrm{I})$. There exists a vertex of $\mathrm{V}$ that eliminates $\overline{\mathrm{uv}} \in \mathrm{I}$ if and only if the node $\mathrm{n}_{\overline{\mathrm{uv}}}$ of the contracted $\mathrm{ElF}$ has a leaf attached to it.

Proof. We start by proving the first direction of the lemma. Let $\mathrm{p} \in \mathrm{V}$ be the closest vertex to the edge $\overline{u v} \in \operatorname{CDT}(\mathrm{I})$ that eliminates $\overline{u v}$. By Lemma 6.3, $\overline{u v}$ belongs to the elimination path induced by $p$. If $\overline{u v}$ is the first edge on this elimination path or every edge on this path between $p$ and $\overline{u v}$ is not in $\mathrm{I}$, then $n_{\overline{u v}}$ has a leaf (that represents this path) in contracted ElF. Assume to the contrary that $n_{\overline{u v}}$ has no immediate leaf, meaning that elimination path from $p$ to $\overline{u v}$ contains an edge of I. By Lemma 6.2 this edge intersects both $\overline{p u}$ and $\overline{p v}$, which contradicts to $p$ being visible to $u$ and $v$.

Let us prove the opposite direction. Let $n_{\overline{u v}}$ be a node of contracted ElF that has a leaf attached to it. We have to prove that there exist a vertex that eliminates $\overline{u v}$. Notice also, that if ElF does not contain node $n_{\overline{u v}}$ then $\overline{u v}$ does not belong to any elimination path. Among all the possible leaves attached to $n_{\overline{u v}}$ let us choose the one that corresponds to the vertex $p$ closest to $\overline{u v}$. According to the construction of ElF, elimination path induced by $p$ (let us call it path $h_{p}$ ) contains $\overline{u v}$. Notice that elimination path represents a list of triangles of $\mathrm{CDT}(\mathrm{I})$, such that each pair of consecutive edges of the path belongs to the same triangle. If $\triangle(p, u, v) \in \operatorname{CDT}(\mathrm{I})$ then $p$ eliminates $\overline{u v}$ and we are done.

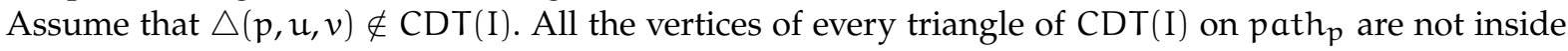
$\triangle(p, u, v)$. Otherwise, the path induced by such a vertex will overlay with path , which contradicts to $p$ being the closest to $\overline{u v}$ among other leaves attached to $n_{\overline{u v}}$. Thus all the edges between $p$ and $\overline{u v}$ on path $_{\mathrm{p}}$ intersect both $\overline{\mathrm{pu}}$ and $\overline{\mathrm{pv}}$. Moreover, none of those edges belong to I, otherwise, in contracted ElF $p$ will not be a leaf of $n_{\overline{u v}}$. It follows that in CDT(I) there does not exist an edge of I that intersects $\overline{p u}$ or $\overline{p v}$. Therefore, $p$ is visible to both $u$ and $v$ and thus $p$ eliminates $\overline{u v}$. 
We are ready to present an algorithm that finds the minimum set $S \subseteq \mathrm{E}$ of edges such that $\mathrm{I} \subseteq$ $\mathrm{CG}_{\beta}(\mathrm{V}, \mathrm{S})$ for constrained $\beta$-skeletons $(1 \leqslant \beta \leqslant 2)$ :

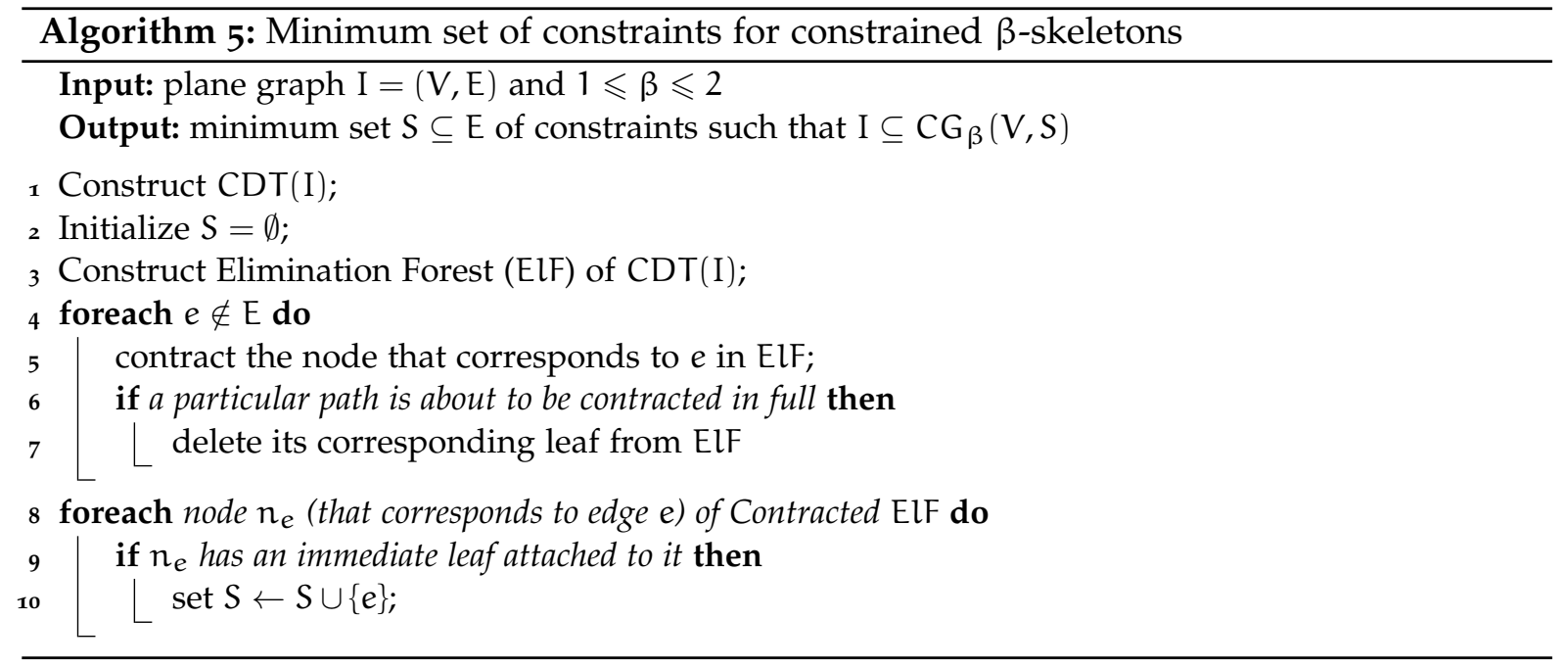

Let us discuss the correctness of Algorithm 5. Let $S$ be the output of the algorithm on the input plane graph $I=(V, E)$. Notice that the following is true: $I \subseteq C_{\beta}(V, S)$. Every edge of $E$ that belongs to $S$ also belongs to $\mathrm{CG}_{\beta}(\mathrm{V}, \mathrm{S})$ by definition. According to the algorithm, every edge of $E \backslash S$ does not have a leaf attached to a corresponding node in contracted ElF. By Lemma 6.4 none of those edges has a vertex that eliminates it.

LEMMA 6.5. Let $\mathrm{S}$ be the output of Algorithm 5 on the input plane graph $\mathrm{I}=(\mathrm{V}, \mathrm{E})$. The set $\mathrm{S}$ is minimum.

Proof. Assume to the contrary that there is another set $S^{\prime} \subseteq \mathrm{E}$, such that $\mathrm{I} \subseteq \mathrm{CG}_{\beta}\left(\mathrm{V}, \mathrm{S}^{\prime}\right)$ and $|S|>\left|\mathrm{S}^{\prime}\right|$. Let $e^{\prime}$ be an edge of $S \backslash S^{\prime}$. Notice, that $e^{\prime} \in \mathrm{I}$. Algorithm 5 added $e^{\prime}$ to $S$, thus there exists a leaf in the contracted $\mathrm{ElF}$ attached to $n_{e^{\prime}}$. By Lemma 6.4 there exists a vertex of $V$ that eliminates $e^{\prime}$. Thus $\mathrm{e}^{\prime} \notin \mathrm{CG}_{\beta}\left(\mathrm{V}, \mathrm{S}^{\prime}\right)$. This contradicts to $\mathrm{I} \subseteq \mathrm{CG}_{\beta}\left(\mathrm{V}, \mathrm{S}^{\prime}\right)$.

The running time of Algorithm 5 depends on the complexity of the first step. Steps $2-10$ can be performed in $\mathrm{O}(n)$ time. In the worst case the construction of $\mathrm{CDT}(\mathrm{I})$ can take $\mathrm{O}(\mathrm{n} \log n)$ time. But for different types of input graph I this time can be reduced. If I is a tree or a polygon, then CDT(I) can be constructed in $\mathrm{O}(\mathrm{n})$ time. If $\mathrm{I}$ is a triangulation, then $\mathrm{I}=\mathrm{CDT}(\mathrm{I})$ and thus the first step is accomplished in $\mathrm{O}(1)$ time.

THEOREM 6.6. Given a plane graph $\mathrm{I}=(\mathrm{V}, \mathrm{E})$, Algorithm 5 constructs the minimum set $\mathrm{S} \subseteq \mathrm{E}$ of constraints such that $\mathrm{I} \subseteq \mathrm{CG}_{\beta}(\mathrm{V}, \mathrm{S})$. The running time of Algorithm 5 is $\mathrm{O}(\mathrm{n} \log \mathrm{n})$, where $\mathrm{n}=|\mathrm{V}|$. 


\section{HIERARCHY}

Proximity graphs form a nested hierarchy, a version of which was established in [45]:

THEOREM 7.I (Hierarchy). In any $\mathrm{L}_{p}$ metric, for a fixed set $\mathrm{V}$ of points and $1 \leqslant \beta \leqslant 2$, the following is true: $\mathrm{MST} \subseteq \mathrm{RNG} \subseteq \mathrm{G}_{\beta} \subseteq \mathrm{GG} \subseteq \mathrm{DT}$.

We show that proximity graphs preserve the above hierarchy in the constraint setting (refer to Lemma 7.2). We also show that the minimum set of constraints required to reconstruct a given plane graph (as a part of each of those neighbourhood graphs) form an inverse hierarchy (refer to Lemma 7.3).

LEMMA 7.2. Given a plane forest $\mathrm{F}=(\mathrm{V}, \mathrm{E})$ and $1 \leqslant \beta \leqslant 2$, $\mathrm{CMST}(\mathrm{F}) \subseteq \mathrm{CRNG}(\mathrm{F}) \subseteq \mathrm{CG}_{\beta}(\mathrm{F}) \subseteq \mathrm{CGG}(\mathrm{F}) \subseteq \mathrm{CDT}(\mathrm{F})$ Given a plane graph $\mathrm{I}=(\mathrm{V}, \mathrm{E})$ and $1 \leqslant \beta \leqslant 2, \mathrm{CRNG}(\mathrm{I}) \subseteq$ $\mathrm{CG}_{\beta}(\mathrm{I}) \subseteq \mathrm{CGG}(\mathrm{I}) \subseteq \mathrm{CDT}(\mathrm{I})$.

Proof. Let us first prove the second statement of the lemma. Assume that we are given a plane graph $I=(V, E)$. We

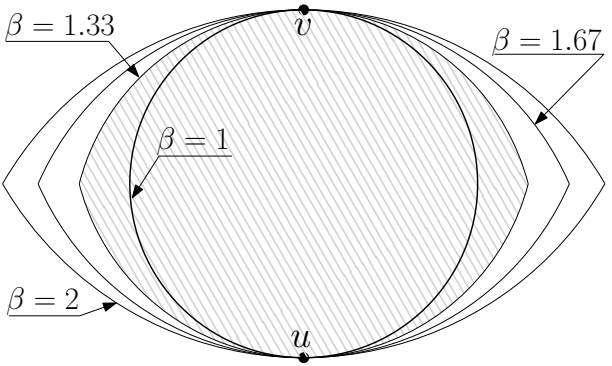

Figure 7.1: Lune-based neighbourhoods for $1 \leqslant \beta \leqslant 2$, where $\mathrm{U}_{\mathfrak{u}, v}(\beta=1.33)$ is dashed. start by proving that $\mathrm{CGG}(\mathrm{I}) \subseteq \mathrm{CDT}(\mathrm{I})$. Let $\overline{u v}$ be an edge of $\mathrm{CGG}(\mathrm{I})$. If $\overline{u v} \in \mathrm{E}$ then $\overline{u v} \in \mathrm{CDT}$ (I) by definition. If $\overline{u v} \notin \mathrm{E}$ then $\overline{u v}$ is locally Gabriel, meaning that the circle $\mathrm{C}_{\mathfrak{u}, v}$ with $\overline{u v}$ as a diameter is empty of points of $V$ visible to both $u$ and $v$. By Delaunay criterion $\overline{u v}$ is a Delaunay edge and thus $\overline{u v} \in \operatorname{CDT}(\mathrm{I})$.

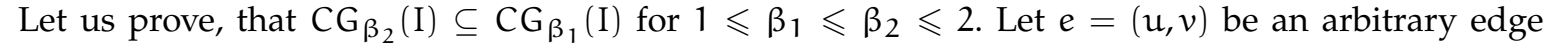
of $\mathrm{CG}_{\beta_{2}}$ (I). If $e \in \mathrm{I}$ then by definition $e \in \mathrm{CG}_{\beta_{1}}$ (I), since $e \in \mathrm{I} \subseteq \mathrm{CG}_{\beta_{1}}$ (I). If $e \notin \mathrm{I}$ then $e$ is not a constraint in $\mathrm{CG}_{\beta_{2}}(\mathrm{I})$ and thus $\mathrm{U}_{\mathfrak{u}, v}\left(\beta_{2}\right)$ does not contain points in $\mathrm{V}$ visible to both $u$ and $v$. By construction, $\mathrm{U}_{\mathfrak{u}, v}\left(\beta_{1}\right) \subseteq \mathrm{U}_{\mathfrak{u}, v}\left(\beta_{2}\right)$, refer to Fig. 7.1. Thus, $\mathrm{U}_{\mathfrak{u}, v}\left(\beta_{1}\right)$ is also empty of points of $V$ visible to both $u$ and $v$. It follows that $e \in \mathrm{CG}_{\beta_{1}}(\mathrm{I})$. We proved the following: $\mathrm{CRNG}(\mathrm{I})=\mathrm{CG}_{\beta=2}(\mathrm{I}) \subseteq$ $\mathrm{CG}_{1 \leqslant \beta \leqslant 2}(\mathrm{I}) \subseteq \mathrm{CG}_{\beta=1}(\mathrm{I})=\mathrm{CGG}(\mathrm{I})$. We are left to prove the first statement of the lemma. Assume that we are given a plane forest $F=(V, E)$. Since $F$ is a plane graph, the second statement of the lemma is true for $F$, so we only need to prove that CMST(F) $\subseteq$ CRNG(F). Let $e=(u, v)$ be an edge of CMST(F). If $e \in F$ then by definition $e \in C R N G(F)$, since $e \in F \subseteq C R N G(F)$. If $e \notin F$ then $e$ is not a constraint in $\mathrm{CMST}(\mathrm{F})$. Assume to the contrary that $e \notin \mathrm{CRNG}(\mathrm{F})$, meaning that the lune $\mathrm{L}_{\mathfrak{u}, v}$ is not "empty". It implies that there is a point $p \in \mathrm{L}_{u, v}$ visible to both $u$ and $v$. Refer to Fig. 6.1a. By construction $|p u|<|v u|$ and $|p v|<|v u|$. Removal of the edge e from CMST(F) disconnects the tree. Assume, without loss of generality, that $v$ and $p$ are in the same connected component of CMST(F) $\backslash\{e\}$. The graph CMST(F) $\backslash\{e\} \cup\{\overline{u p}\}$ is a tree. Moreover, $w(\operatorname{CMST}(\mathrm{F}))>w(\mathrm{CMST}(\mathrm{F}) \backslash\{\boldsymbol{e}\} \cup\{\overline{\mathrm{up}}\})$. This is a contradiction to $\mathrm{CMST}(\mathrm{F})$ being a constrained minimum spanning tree of $F$ and thus $e \in C R N G(F)$.

LEMMA 7.3. Let $S_{\mathrm{G}}$ denote the the minimum set of constraints of $\mathrm{G}$. Given a plane graph $\mathrm{I}=(\mathrm{V}, \mathrm{E})$ and $1 \leqslant \beta \leqslant 2, S_{C R N G(I)} \supseteq S_{C_{\beta}(I)} \supseteq S_{C G G(I)} \supseteq S_{C D T(I)}$. Given a plane forest $F=(V, E)$ and $1 \leqslant \beta \leqslant 2$, $S_{C M S T(F)} \supseteq S_{C R N G(F)} \supseteq S_{C_{\beta}(F)} \supseteq S_{C G G(F)} \supseteq S_{C D T(F)}$.

Proof. First we prove that $\mathrm{S}_{\mathrm{CDT}(\mathrm{I})} \subseteq \mathrm{S}_{\mathrm{CGG}(\mathrm{I})}$. Let $e=(u, v)$ be a constraint in CDT(I), namely, $e \in \mathrm{S}_{\mathrm{CDT}(\mathrm{I})}$. It follows, that $e \in \mathrm{I}$ and $e$ is not locally Delaunay and thus there exist a pair of triangles in $\operatorname{CDT}(\mathrm{I}): \triangle(u, v, p)$ and $\triangle(u, v, q)$, such that $C_{\triangle(u, v, p)}$ contains $q$ or $C_{\triangle(u, v, q)}$ contains $p$, where $C_{\triangle}$ is a circle circumscribing specified triangle. For example, refer to Fig. 7.2, where $C_{\triangle(u, v, p)}$ contains q. 


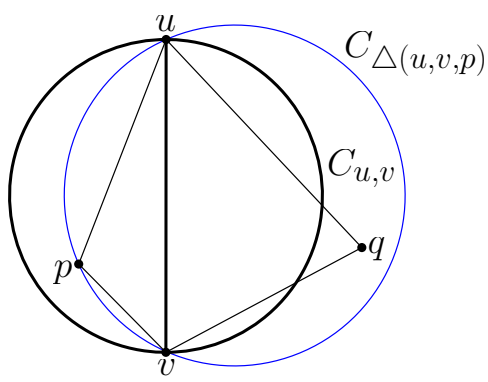

Figure 7.2: $\triangle(u, v, p)$ and $\triangle(u, v, q)$ are triangles of $\operatorname{CDT}(\mathrm{I})$. The circle $C_{\triangle(u, v, p)}$ contains $q$ - the edge $\overline{u v}$ is not locally Delaunay.

Consider the quadrilateral $\{u, p, v, q\}$. At least one of the angles $\angle v p u$ or $\angle u q v$ is obtuse. The following is true for the apex of the obtuse angle:

1. It is contained in the circle $\mathrm{C}_{\mathfrak{u}, v}$ with the edge $e$ as a diameter.

2. It is visible to both $u$ and $v$ in $\mathrm{CDT}(\mathrm{I})$, since together with $u$ and $v$ it creates a triangle that belongs to $\mathrm{CDT}(\mathrm{I})$. It is also visible to both $u$ and $v$ in $\mathrm{CGG}(\mathrm{I})$, because $\mathrm{CGG}(\mathrm{I}) \subseteq \mathrm{CDT}(\mathrm{I})$, refer to Lemma 7.2.

The above makes the edge $e$ not locally Gabriel. Since $e \in I$, it must belong to CGG(I), and thus $e \in \mathrm{S}_{\mathrm{CGG}(\mathrm{I})}$. Therefore $\mathrm{S}_{\mathrm{CDT}(\mathrm{I})} \subseteq \mathrm{S}_{\mathrm{CGG}(\mathrm{I})}$.

Notice that $S_{C_{\beta_{\beta_{1}}}(\mathrm{I})} \subseteq S_{\mathrm{CG}_{\beta_{2}}(\mathrm{I})}$ for $1 \leqslant \beta_{1} \leqslant \beta_{2} \leqslant 2$ since $\mathrm{U}_{\mathrm{u}, v}\left(\beta_{1}\right) \subseteq \mathrm{U}_{\mathrm{u}, v}\left(\beta_{2}\right)$ for every pair of vertices $u, v \in I$ (refer to Fig. 7.I). This completes the proof of the first statement of the lemma, which also holds if $I$ is a forest $F$. Thus, we only left to prove that, given a plane forest $F=(V, E)$, $S_{C R N G(F)} \subseteq S_{C M S T(F)}$. Let $e=(u, v)$ be an edge in $S_{C R N G(F)}$, meaning that $e \in F$ and $e$ is a constraint in CRNG(F). Thus, there exists a point $p$ visible to both $u$ and $v$ in $C R N G(F)$, such that $p \in L_{u, v}$. By construction $|p u|<|v u|$ and $|p v|<|v u|$. Refer to Fig. 6.1a. Since $e \in F$ we have $e \in C M S T(F)$. Assume to the contrary, that $e \in C M S T(F)$ but not a constraint in CMST $(F)$. Removal of e from CMST(F) disconnects the tree. Assume, without loss of generality, that $v$ and $p$ are in the same connected component of CMST $(\mathrm{F}) \backslash\{e\}$. The graph CMST(F) $\backslash\{e\} \cup\{\overline{\mathrm{up}}\}$ is a tree and its weight is smaller then the weight of $\mathrm{CMST}(\mathrm{F})$. This is a contradiction to $\mathrm{CMST}(\mathrm{F})$ being a constrained minimum spanning tree of $F$ and thus e must be assigned weight 0 and be a constraint in $\mathrm{CMST}(\mathrm{F})$. 
We have considered geometric compression of neighbourhood graphs, such as minimum spanning trees, relative neighbourhood graphs, Gabriel graphs and $\beta$-skeletons for $1 \leqslant \beta \leqslant 2$, in the constraint setting.

We have presented an $\mathrm{O}(|\mathrm{V}| \log |\mathrm{V}|)$-time algorithm that finds the minimum set $S \subseteq \mathrm{E}$ of edges of a given plane forest $F=(V, E)$ such that $F \subseteq \operatorname{CMST}(V, S)$. It would be interesting to decide if the running time of our algorithm is optimal, by showing a lower bound of $\Omega(|\mathrm{V}| \log |\mathrm{V}|)$ on the number of steps that any algorithm must perform in order to solve this problem.

We have also presented an algorithm for finding the minimum set $S \subseteq E$ of edges of a given plane graph $I=(V, E)$ such that $I \subseteq C_{\beta}(V, S)$ for $1 \leqslant \beta \leqslant 2$. For the case when $\beta=2$, we presented a much simpler algorithm. The running time of both algorithms is $\mathrm{O}(|\mathrm{V}|)$, provided that the constrained Delaunay triangulation of $\mathrm{I}$ is given.

We have not considered $\beta$-skeletons for values of $\beta$ that are outside the interval $[1,2]$, because they are not in the scope of the hierarchy established by Toussaint (refer to Theorem 7.1) and they are not subgraphs of the Delaunay triangulation.

We have shown that the neighbourhood graph that contains $(\mathrm{V}, \mathrm{E})$ can be successfully reconstructed from $V$ and $S$. It would be interesting to estimate the effectiveness of our geometric compression algorithms on real data sets.

We believe, that our approach for finding the minimum set of constraints can also be used for metrics other than Euclidean metrics. 


\section{Part II}

PROBING CONVEX POLYGONS WITH A WEDGE 


\section{INTRODUCTION}

Geometric probing is a branch of computational geometry looking into how to determine shapes of unknown geometric objects by using special measurements - probes. It is an area of research with various applications in robotics, automated manufacturing and tomography to mention only a few. Geometric probing was introduced by Cole and Yap [14] in 1983. Many probing tools, together with reconstruction algorithms, have been developed - finger probes [14], hyperplane (or line) probes [18, 34], diameter probes [38], x-ray probes [19, 23], histogram (or parallel x-ray) probes [36], half-plane probes [42], composite probes [10, 34, 40] among others. Another closely related area, called geometric testing, studies a verification problem: find a set of probes to determine if a given set of geometric objects contains one which is equivalent to a certain query object (see [39] for a survey).

Table 9.1: Summary of results for reconstructing convex polygons. $N_{B}$ stands for the number of angles smaller than $\omega$ in the convex polygon.

\begin{tabular}{l|c|c|c|c}
\hline \hline Probing Tool & Notes & Lower & Upper & References \\
\hline \hline finger & & $3 n-1$ & $3 n$ & {$[14]$} \\
\hline hyperplane/line & & $3 n+1$ & $3 n+1$ & {$[34]$} \\
\hline diameter & Reconstruction is not possible & $*$ & $*$ & {$[38]$} \\
\hline x-ray & & $2 n$ & $5 n+19$ & {$[19]$} \\
\hline half-plane & & $2 n$ & $7 n+7$ & {$[42]$} \\
\hline & $\omega=\pi / 2, N_{B}=0$ & $2 n-3$ & $2 n-3$ & \\
& $0<\omega<\pi / 2, N_{B}=0$ & $2 n-2$ & $2 n-2$ & \\
$\omega$-wedge & $0<\omega \leqslant \pi / 2, N_{B}=1$ & $2 n-1$ & $2 n-1$ & our results \\
& $0<\omega \leqslant \pi / 2, N_{B}=2$ & $2 n+2$ & $2 n+3$ & \\
& $0<\omega \leqslant \pi / 2, N_{B}=3$ & $2 n+2$ & $2 n+5$ & \\
\hline
\end{tabular}

Various probing schemes along with upper and lower bounds on performance are summarized in the upper part of Table 9.I (see the review paper of Skiena [41], discussing the methodology as well the challenges of geometric probing).

For a given probing tool, we seek to reconstruct an object with as few probes as possible; hence much effort has gone to finding an algorithm with sufficiently tight upper and lower bounds or, ultimately, an optimal algorithm. We introduce a new probing tool - an $\omega$-wedge. It consists of two rays emanating from a point called the apex of the wedge, forming an angle $\omega$, where $\omega$ is a fixed real number with $0<\omega \leqslant \pi / 2$. To probe a convex polygon $\mathcal{O}$ we choose a directed line $\overrightarrow{\mathrm{L}}$ and position the apex of the $\omega$-wedge on $\vec{L}$ such that $\mathcal{O}$ and the positive direction of $\overrightarrow{\mathrm{L}}$ are contained in the wedge. Initially, $\mathcal{O}$ does not touch the rays of the wedge. Informally, imagine the $\omega$-wedge moving from its initial position along and in the direction of $\overrightarrow{\mathrm{L}}$ until both rays contact $\mathcal{O}$ in which case the wedge cannot move any further. A valid $\omega$-probe of a convex polygon $\mathcal{O}$ is a placement of an $\omega$-wedge, such that $\mathcal{O}$ is contained in the wedge and touches both of its rays. An outcome of a valid $\omega$-probe consists of the coordinates of the apex, the orientation of both rays and the coordinates of the points of contact between $\mathcal{O}$ and each of the rays closest to the apex. Our probing tool generalizes previous work (see 
e.g., [4], [21]). Our method of angular probing was inspired by problems on enclosing triangles [4], namely searching for all $\omega$-angle triangles of minimum area which enclose a given set of points in the plane. At the same time, the way of probing with a wedge can be thought of as a photographer locating himself and orienting his camera such that $\mathcal{O}$ fits exactly in the field of view of his camera [21].

We present an algorithm that reconstructs a convex n-gon with all internal angles of size bigger than $\omega$ using $2 n-2 \omega$-probes (for $0<\omega<\pi / 2$ ). When $\omega=\pi / 2$, the reconstruction uses $2 n-3 \omega$-probes. We prove optimality for both cases. We show that $2 n-1$ probes are necessary and sufficient to reconstruct a convex polygon with exactly one vertex whose internal angle is at most $\omega$. The main difficulty that is common to reconstruction problems, both in theory and in practice, is the reconstruction of sharp corners (or sharp edges if we talk about 3 dimensions); see, e.g., [1]. Those areas of so called high-curvature cause difficulties for our probing tool as well. When the polygon has 2 or 3 angles smaller than or equal to $\omega$, our results are almost optimal. In particular, for polygons with exactly 2 (respectively, 3) angles of size at most $\omega$, we show a reconstruction strategy that uses $2 \mathfrak{n}+3$ (respectively, $2 n+5) \omega$-probes, while $2 n+2$ probes are necessary. Our results are summarized in Table 9.1. Our reconstruction strategies are adaptive, i.e., the choice of parameters for each probe depends on all previous outcomes. We also prove lower bounds for all of our algorithms using adversarial arguments.

Our probing tool is similar to finger probing [14], where each probe is formally defined to be a directed line $\overrightarrow{\mathrm{L}}$. In finger probing there is a point moving along and in the direction of $\overrightarrow{\mathrm{L}}$ until it contacts $\mathcal{O}$. In our research the point is equipped with two rays. The movement of the apex $q$ along $\vec{L}$ terminates when both rays contact $\mathcal{O}$ ( $q$ does not necessarily touch $\mathcal{O}$ ). Our model of probing achieves better results than finger probing (consider Table 9.1) mainly because the outcome of a valid $\omega$-probe contains one or two points of contact with $\mathcal{O}$ (which are vertices of $\mathcal{O}$ ), while in finger probing the outcome of a successful probe is a single point (not necessarily a vertex of $\mathcal{O}$ ). Moreover, almost every edge of a polygon, reconstructed via finger probing, contains two points of contact in its interior. In our model only one probe is sufficient to verify an edge.

Our model of probing should not be confused with diameter or "parallel-jaw gripper" model of probing [38], where the shape of a polygon is determined from a sequence of projections. Rao and Goldberg showed that reconstruction of $\mathcal{O}$ with a diameter probing tool is not possible, - for a given set of diameter measurements, there is an infinite set of polygonal shapes consistent with these measurements. We define the angle between the rays of our probing tool to be strictly bigger than 0 . Thus, the rays of an $\omega$-wedge are never parallel. Another difference is that a valid $\omega$-probe returns points of contact with $\mathcal{O}$, while a diameter probe does not, - it returns only the distance between supporting lines.

Our probing tool is quite simple compared to other probing devices such as x-ray or histogram. It can be manufactured from two tactile whisker-like sensors and does not require complicated software.

The organization of this part of the thesis is as follows. In Chapter 10 we present definitions related to our probing method. In Chapter II we give some observations, review known facts and prove some fundamental properties related to $\omega$-probing. We concentrate on the reconstruction of convex polygons in Chapters 12 and 13. In Chapter 12 we present an optimal algorithm for reconstructing convex polygons that do not have narrow vertices (refer to Definition 10.6). Reconstruction of general convex polygons together with the discussion on upper and lower bounds on the performance are given in Chapter 13. Concluding remarks and possible directions for future research are presented in Chapter 14. 


\section{DEFINITIONS}

Given a convex polygon $\mathcal{O}$ in the plane, the goal is to probe $\mathcal{O}$ to determine its exact shape and orientation with a minimum number of probes. We probe $\mathcal{O}$ with a wedge $W$ consisting of two infinite arms making an angle of $\omega$ (for some fixed angle $0<\omega \leqslant \pi / 2$ ). An $\omega$-probe of $\mathcal{O}$ returns the coordinates of the apex of $W$ together with the orientation of the arms and one point of contact of each arm with $\mathcal{O}$, such that the returned point of contact is the point of $\mathcal{O}$ that is closest to the apex. This is formalized in the following definitions. For the rest of this part of the thesis, $\mathcal{O}$ is a convex polygon in the plane and $\omega$ is an angle such that $0<\omega \leqslant \pi / 2$. In Chapter 14 we discuss the case where $\pi / 2<\omega<\pi$.

DEFINITION IO.I ( $\omega$-Wedge). Let $\mathrm{q}$ be a point in the plane. Let $\mathrm{H}_{1}$ and $\mathrm{H}_{2}$ be two rays emanating from $\mathrm{q}$ such that the angle between $\mathrm{H}_{1}$ and $\mathrm{H}_{2}$ is $\omega$ and $\left(\mathrm{q}, \mathrm{h}_{1}, \mathrm{~h}_{2}\right)$ forms a left-turn (where $\mathrm{h}_{1}, \mathrm{~h}_{2}$ are points different from $\mathrm{q}$ such that $\mathrm{h}_{1} \in \mathrm{H}_{1}, \mathrm{~h}_{2} \in \mathrm{H}_{2}$ ). The closed set formed by $\mathrm{q}, \mathrm{H}_{1}, \mathrm{H}_{2}$ and the points between $\mathrm{H}_{1}$ and $\mathrm{H}_{2}$ is an $\omega$-wedge, denoted $\mathcal{W}\left(\omega, \mathrm{q}, \mathrm{H}_{1}, \mathrm{H}_{2}\right)$. The point $\mathrm{q}$ is the apex of the $\omega$-wedge. (refer to Figure 10.1a).

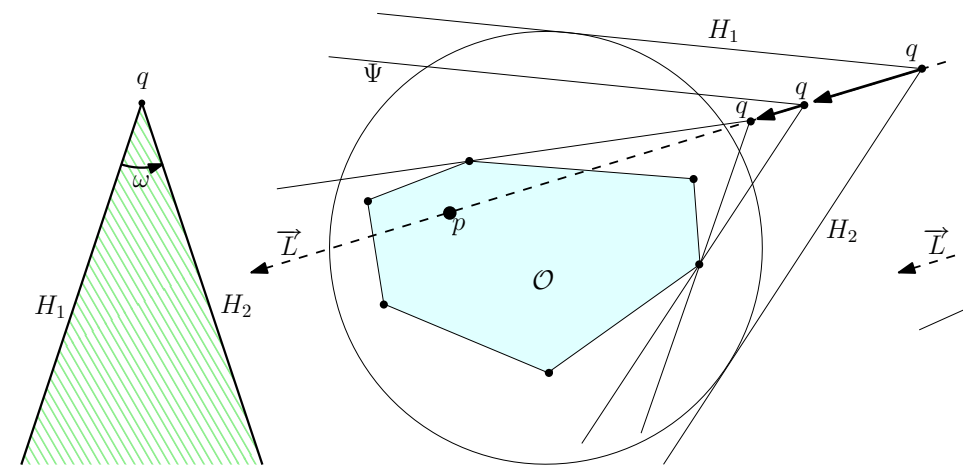

(a)

(b)

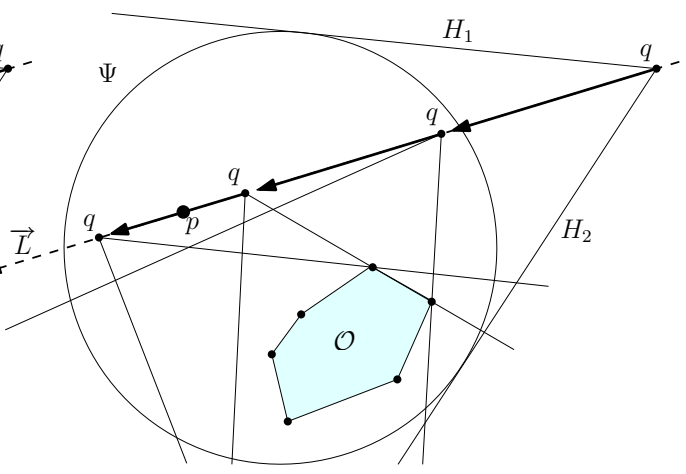

(c)

Figure 10.1: Definition and overview of the probing tool. We assume that we know a point $p$ and a circle $\Psi$ such that $p \in \mathcal{O}$ and $\mathcal{O} \subset \Psi$. (a) $\omega$-wedge; (b) Probing along a directed line $\overrightarrow{\mathrm{L}}$ that intersects $\mathcal{O}$; (c) Despite the fact that $\mathcal{O}$ is inside $W$, the probe will miss $\mathcal{O}$ because $p \notin \mathcal{O}$.

To probe with an $\omega$-wedge, we set the direction that the apex $q$ of the probe has to follow, the line $\mathrm{L}$, and the initial orientation of the probe. As the apex $q$ of the probe moves along $L$, the arms can move freely provided that they maintain an angle of $\omega$ and $\mathcal{O}$ stays between the arms. We do not have any control over rotation of the arms. When q cannot move anymore, then we have a valid $\omega$-probe of $\mathcal{O}$ (refer to Definition 10.2).

DEFINItion 10.2 (valid $\omega$-Probe of $\mathcal{O}$ ). A valid $\omega$-probe of $\mathcal{O}$ is a quadruple $\left(\omega, \mathrm{q}, \mathrm{H}_{1}, \mathrm{H}_{2}\right)$ such that $W=\mathcal{W}\left(\omega, \mathrm{q}, \mathrm{H}_{1}, \mathrm{H}_{2}\right)$ is an $\omega$-wedge; $\mathcal{O} \subseteq W ; \mathrm{H}_{1}$ (respectively $\mathrm{H}_{2}$ ) contains at least one point of $\mathcal{O}$.

DEFINITION 10.3 (Probing along a directed line $\overrightarrow{\mathrm{L}}$ ). Let $\overrightarrow{\mathrm{L}}$ be a directed line and $\left(\omega, \mathrm{q}, \mathrm{H}_{1}, \mathrm{H}_{2}\right)$ be a valid $\omega$-probe of $\mathcal{O}$ such that the apex $\mathrm{q}$ is on $\overrightarrow{\mathrm{L}}$, and the ray from $\mathrm{q}$ in the direction of $\overrightarrow{\mathrm{L}}$ intersects $\mathcal{O}$. We say that $\left(\omega, \mathrm{q}, \mathrm{H}_{1}, \mathrm{H}_{2}\right)$ is a valid $\omega$-probe of $\mathcal{O}$ with respect to $\overrightarrow{\mathrm{L}}$. Refer to Figure 10.1b.

We assume that we know a point $p$ and a circle $\Psi$ such that $p \in \mathcal{O}$ and $\mathcal{O} \subset \Psi$. The hypothesis $\mathcal{O} \subset \Psi$ guarantees that $\mathcal{O}$ is inside $W$ (refer to [21] for instance). The hypothesis $p \in \mathcal{O}$ guarantees that $H_{1}$ and 
$\mathrm{H}_{2}$ will eventually touch $\mathcal{O}$ as q approaches $p$ (refer to [14] for instance). Otherwise, we could miss the object. Indeed, suppose that for some probe we choose a point $p \in \Psi \backslash \mathcal{O}$ to aim at. Suppose that we choose a direction $\overrightarrow{\mathrm{L}}$ containing $p$ that does not intersect $\mathcal{O}$ (refer to Figure 10.1c). As $q$ moves along $\overrightarrow{\mathrm{L}}, \mathrm{H}_{2}$ will eventually touch $\mathcal{O}$. Then $\mathrm{H}_{2}$ will turn counter-clockwise around $\mathcal{O}$ as q goes further. But q can go forever along $\overrightarrow{\mathrm{L}}$ and $\mathrm{H}_{1}$ will never touch $\mathcal{O}$. So we miss $\mathcal{O}$ even though $\mathcal{O}$ is inside $W$. This shows that for every probe, we need to aim towards a point in $\mathcal{O}$.

DEFINITION 10.4 (Outcome of probing along a directed line $\overrightarrow{\mathrm{L}}$ ). The outcome of probing with an $\omega$ wedge along a directed line $\overrightarrow{\mathrm{L}}$ consists of the following values: $\mathrm{q}, \mathrm{H}_{1}, \mathrm{H}_{2}, \mathrm{p}_{1}$ and $\mathrm{p}_{2}$, such that $\mathcal{W}\left(\omega, \mathrm{q}, \mathrm{H}_{1}, \mathrm{H}_{2}\right)$ is a valid $\omega$-probe and the point $\mathrm{p}_{1}$ (respectively $\mathrm{p}_{2}$ ) is the point of $\mathcal{O} \cap \mathrm{H}_{1}$ (respectively $\mathcal{O} \cap \mathrm{H}_{2}$ ) that is closest to $\mathrm{q}$.

Notice that $p_{1}$ is on or to the right of $\vec{L}, p_{2}$ is on or to the left of $\vec{L}$ and we can also have $p_{1}=p_{2}=q$ (refer to Figure 10.2C).

Consider three consecutive vertices $v_{i+1}, v_{i}$ and $v_{i-1}$ of $\mathcal{O}$ such that $\angle\left(v_{i+1}, v_{i}, v_{i-1}\right)<\omega$. (refer to Figure $10.2 \mathrm{C}$ ). If $\mathcal{O}$ is contained in the $\omega$-wedge $W$ and the apex $q$ of $W$ is equal to $v_{i}$, then there are infinitely many different probes with $q=v_{i}$. We say that $W$ encloses $\mathcal{O}$ even if $\mathrm{H}_{1}$ and $\mathrm{H}_{2}$ touch $\mathcal{O}$ only at $\mathrm{q}=v_{i}$. This is precisely the situation that causes difficulty since a probe returning a value of $\mathrm{q}=v_{\mathrm{i}}$ gives no additional information.

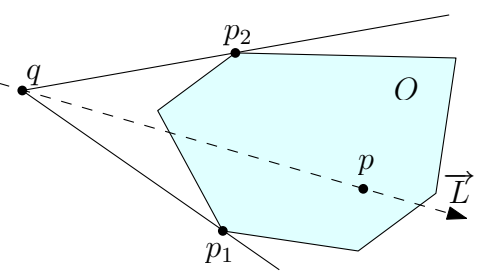

(a) The probe $W$ touches the polygon $\mathcal{O}$ at its vertices.

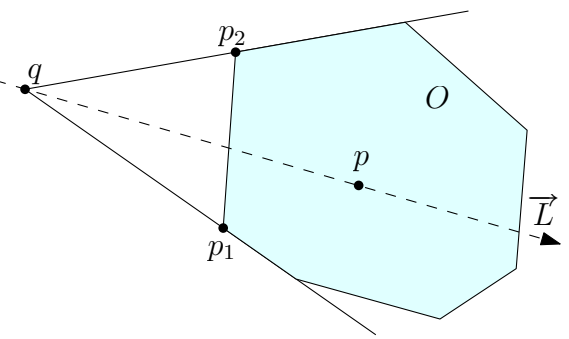

(b) One or more polygon edges coincide with $\mathrm{H}_{1}$ and $\mathrm{H}_{2}$. Only the points of contact $\mathrm{p}_{1}$ and $\mathrm{p}_{2}$ closest to $\mathrm{q}$ are reported.

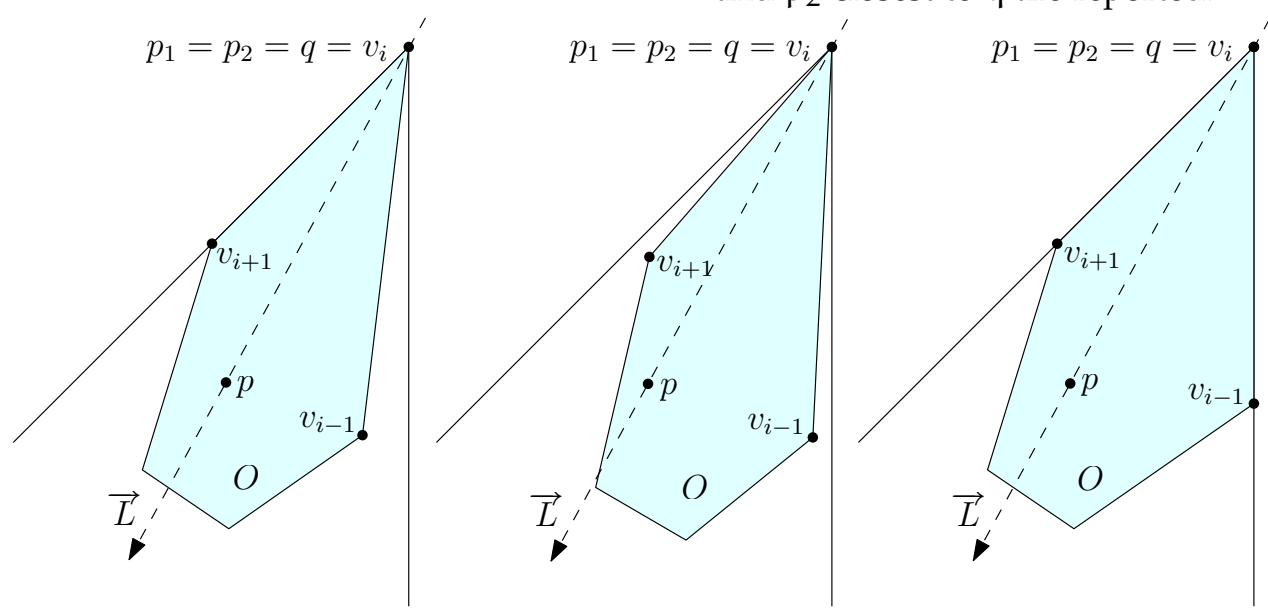

(c) The apex $q$ of the probe touches $\mathcal{O}$. In these cases $q$ is considered to be the only point of contact with the polygon.

Figure 10.2: Possible outcomes of the probing regarding orientation and shape of the polygon.

In this thesis we study the reconstruction of $\mathcal{O}$ by using an $\omega$-wedge model of probing. When it is clear from the context, we write "probe" instead of $\omega$-probe. 
DEFINITION 10.5 ( $\omega$-Cloud). By rotating an $\omega$-wedge around $\mathcal{O}$, such that it contains $\mathcal{O}$ and both its rays are touching $\mathcal{O}$, the apex traces a sequence of circular arcs. This sequence is called an $\omega$-cloud, denoted $\Omega$ (refer to Figure 10.3). In other words, an $\omega$-cloud is the set of apices of all the valid $\omega$-probes of $\mathcal{O}$. The circular arcs of $\Omega$ are labelled in counterclockwise order by $\Gamma_{j}$ for $0 \leqslant j \leqslant n^{\prime}-1$. We note that $n^{\prime}=O(n)$ [3]. The intersection point of a pair of consecutive circular arcs is called a pivot point.

Each point $x$ on the $\omega$-cloud has the property that there exists a valid $\omega$-probe with the apex placed at $x$. Moreover, if $x$ is not on the polygon, then this probe is unique (refer to Lemma 11.4).

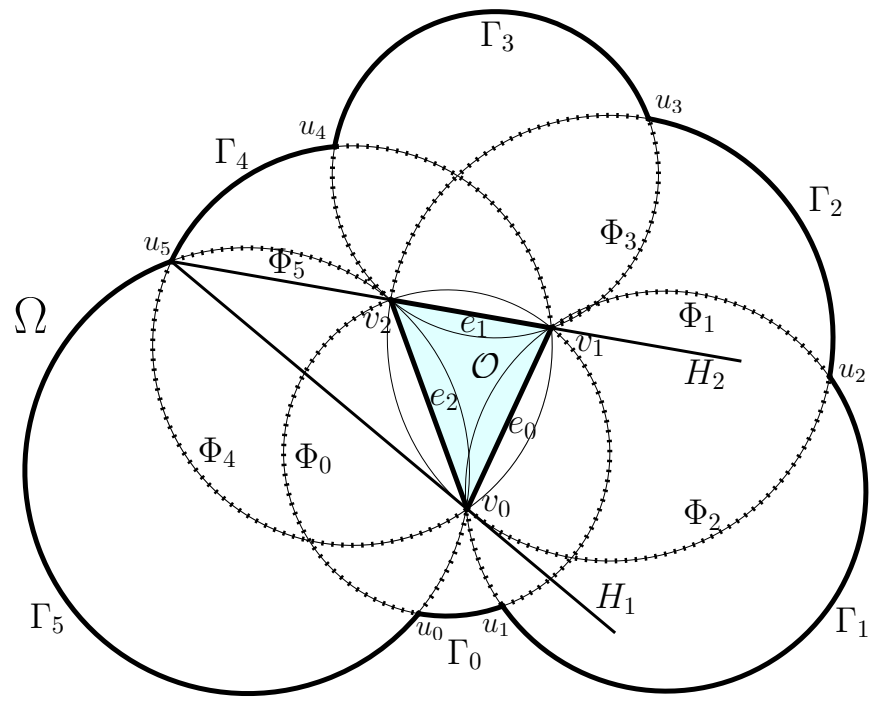

Figure 10.3: $\Omega$ is the $\frac{\pi}{6}$-cloud of $\mathcal{O}=\triangle\left(v_{0}, v_{1}, v_{2}\right)$. A black line represents a circular arc of $\Omega$. A dotted line represents the remaining part of the corresponding $\frac{\pi}{6}$-arc. A grey line represents the remaining part of the corresponding circle.

If a convex polygon $\mathcal{O}$ is initially contained within $W$, the movement of $q$ on $\vec{L}$ will stop when both $\mathrm{H}_{1}$ and $\mathrm{H}_{2}$ have a non-empty intersection with $\mathcal{O}$. There are two cases to consider:

Case 1: $q$ is not on $\mathcal{O}$. In this case, both arms $\mathrm{H}_{1}$ and $\mathrm{H}_{2}$ touch the convex polygon $\mathcal{O}$ at points $p_{1} \in H_{1}$ and $p_{2} \in H_{2}$, such that $p_{1} \neq p_{2}$ (which implies: $p_{1} \neq q$ and $p_{2} \neq q$ ). The apex of the probe $q$ cannot move any further and the probe outputs the result together with the coordinates of the apex (which is a point of the $\omega$-cloud of $\mathcal{O}$ ).

Case 2: $q$ is on $\mathcal{O}$. In the second case, when $q$ touches $\mathcal{O}$ ( $q$ can touch $\mathcal{O}$ only at its vertices), the arms $\mathrm{H}_{1}$ and $\mathrm{H}_{2}$ of $\mathrm{W}$ may or may not touch $\mathcal{O}$ at points other than $\mathrm{q}$. In fact, since the arms $\mathrm{H}_{1}$ and $\mathrm{H}_{2}$ are free to rotate around $q$, if the internal angle of $\mathcal{O}$ at the vertex touched by $q$ is smaller than $\omega$, then there are infinitely many different positions for $W$ (refer to the first two examples of Figure 10.2c). If $q$ touches a vertex whose internal angle is $\omega$, there is only one position for $W$ (refer to the last example of Figure 10.2c). Since we know neither $\mathcal{O}$ nor the $\omega$-cloud $\Omega$ of $\mathcal{O}$, it is not clear whether the arms $\mathrm{H}_{1}$ and $\mathrm{H}_{2}$ touch $\mathcal{O}$ at points other than q. We explain how to deal with this technicality in Chapter 13. In this case, since $0<\omega \leqslant \pi / 2$, q is a vertex of $\mathcal{O}$ and a pivot point of the $\omega$-cloud $\Omega$ of $\mathcal{O}$.

Definition 10.6 (Narrow Vertex). A vertex of the convex polygon $\mathcal{O}$ is called a narrow vertex if its internal angle is at most $\omega$.

If the ray in the direction of an $\omega$-probe enters $\mathcal{O}$ via a narrow vertex $v_{\mathrm{B}}$, then the apex of this probe is on a vertex $v_{\mathrm{B}}$ of $\mathcal{O}$. Refer to Figure 10.2c. However, there can be no more than 3 vertices on the polygon where this happens (refer to Observation 11.1). 
DEFINITION $10.7(\omega$-Arc). Let $\mathrm{a}$ and $\mathrm{b}$ be two points on a circle $\Phi$. Denote by $\Gamma=\widehat{\mathrm{ba}}$ the counterclockwise circular arc from $\mathrm{b}$ to $\mathrm{a}$. By elementary geometry, for any point $\mathrm{q} \in \Gamma, \angle(\mathrm{a}, \mathrm{q}, \mathrm{b})=\frac{\widehat{\mathrm{ab}}}{2}$. (Here and throughout this part of the thesis we use counterclockwise angle naming convention). If $\angle(a, q, b)=\frac{\widehat{a b}}{2}=w$, we say that $\Gamma$ is an $\omega$-arc with respect to $\mathrm{a}$ and $\mathrm{b}$. The points $\mathrm{a}$ and $\mathrm{b}$ are called the supporting points of $\Gamma$.

Therefore, given two distinct points $a$ and $b$, there are two possible $\omega$-arcs: the one with respect to $a$ and $b$ and the one with respect to $b$ and $a$.

DEFINITION 10.8 (Feasible). Let $\mathrm{Q}$ be a convex polygon. Let e be an edge of $\mathrm{Q}$. Let $\mathcal{W}$ be the set of all $\omega$-wedges, each one containing $\mathrm{Q}$. The feasible region $\mathrm{F}_{\mathrm{Q}}$ of $\mathrm{Q}$ is the intersection of all wedges in $\mathcal{W}$. For any edge e of $\mathrm{Q}$, let $\mathrm{H}_{e}$ be the half-plane containing e on its boundary that does not contain $\mathrm{Q}$. The feasible region $\mathrm{F}_{\mathrm{Q}, \mathrm{e}}$ is defined as $\mathrm{F}_{\mathrm{Q}} \cap \mathrm{H}_{\mathrm{e}}$. (refer to Figure 10.4).

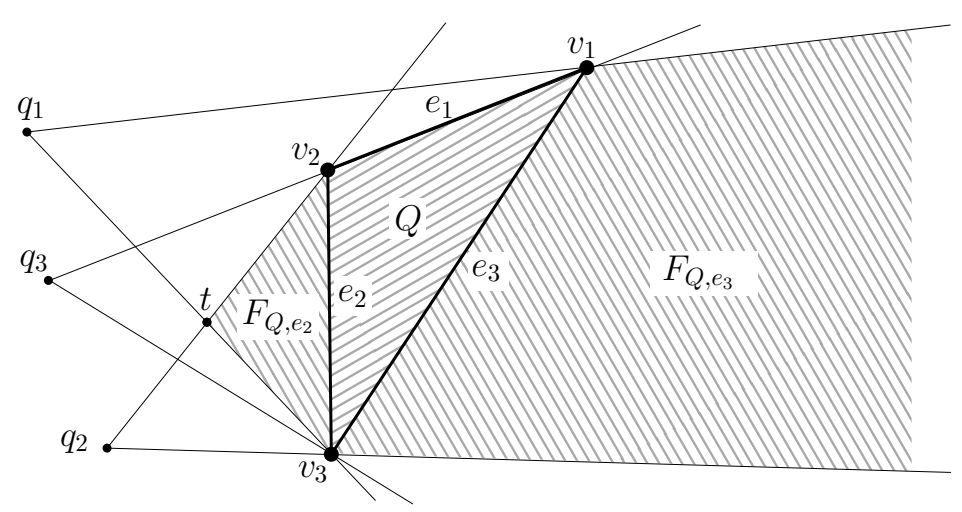

Figure 10.4: $W$ consists of three $\omega$-wedges, each one containing $Q=\left\{v_{1}, v_{2}, v_{3}\right\}$. The feasible region $\mathrm{F}_{\mathrm{Q}}$ is shown in a tiling pattern. The feasible region $\mathrm{F}_{\mathrm{Q}_{,} e_{2}}$ of the edge $e_{2}$ is the triangle $\triangle\left(v_{2}, t, v_{3}\right) . \mathrm{F}_{\mathrm{Q}, \mathrm{e}_{1}}$ degenerates into the line segment $\overline{v_{1} v_{2}}$; in other words, $\mathrm{F}_{\mathrm{Q}, \mathrm{e}_{2}}=\mathrm{e}_{1}$ and thus $e_{1} \in \mathcal{O} . \mathrm{F}_{\mathrm{Q}, e_{3}}$ is unbounded. 


\section{PRELIMINARIES}

\section{1}

When an $\omega$-probe is shot along a directed line that enters $\mathcal{O}$ via a narrow vertex $v_{\mathrm{B}}$, the probe stops only when the apex touches $v_{\mathrm{B}}$ (refer to Figure 10.2C). However, there can be no more than 3 vertices on a convex polygon, that is not a rectangle, where this happens (refer to Observation 11.1), since the angle of an $\omega$-probe is $0<\omega \leqslant \pi / 2$. The number of acute angles is 4 when $\mathcal{O}$ is a rectangle and cannot exceed 3 otherwise.

OBSERVATION 11.1. If $\mathcal{O}$ is a convex polygon that is not a rectangle, then the number of narrow vertices cannot exceed 3 .

Proof. We prove that the maximum number of acute angles of a convex polygon is 3. The sum of the exterior angles of any convex polygon is $2 \pi$. If an inner angle is acute, then the corresponding exterior angle is greater than $\pi / 2$. Suppose that 4 or more acute angles are possible. Since the polygon is convex, all the exterior angles are positive, so the sum of the exterior angles is at least the sum of the exterior angles supplementary to the four or more acute angles, which is strictly greater than $4 \pi / 2=2 \pi$, which is a contradiction.

OBSERVATION 11.2. If $\mathcal{O}$ is a convex polygon that is not a rectangle and $\omega<\pi / 3$, then the number of narrow vertices is at most 2.

Proof. Assume that $\mathcal{O}$ is not a rectangle and has three narrow vertices $v_{\mathrm{B}_{1}}, v_{\mathrm{B}_{2}}$ and $v_{\mathrm{B}_{3}}$, that define a triangle $\triangle\left(v_{\mathrm{B}_{1}}, v_{\mathrm{B}_{2}}, v_{\mathrm{B}_{3}}\right)$. At least one of the angles of the triangle is bigger or equal to $\pi / 3$. Since the triangle completely resides inside $\mathcal{O}$, at least one of the internal angles of $\mathcal{O}$ at $\nu_{\mathrm{B}_{1}}, v_{\mathrm{B}_{2}}$ or $v_{\mathrm{B}_{3}}$ should be bigger or equal to $\pi / 3$. Thus if $\omega<\pi / 3$ then it is not possible for $\mathcal{O}$ to have three narrow vertices.

LEMMA 11.3. Assume that we are given a directed line $\overrightarrow{\mathrm{L}}$. The apices of all valid $\omega$-probes of $\mathcal{O}$ along $\overrightarrow{\mathrm{L}}$ are located at the same point in the plane.

Proof. Assume to the contrary that there are two different valid $\omega$-probes of $\mathcal{O}$ along $\overrightarrow{\mathrm{L}}$ with apices located at different places. We denote them by $\left(q, H_{1}, H_{2}, p_{1}, p_{2}\right)$ and $\left(q^{\prime}, H_{1}^{\prime}, H_{2}^{\prime}, p_{1}^{\prime}, p_{2}^{\prime}\right)$ and we have $q \neq q^{\prime}$. Since $q \in \vec{L}$ and $q^{\prime} \in \vec{L}$, the apex of one of the probes is contained in the $\omega$-wedge of the other probe. Assume, without loss of generality, that the apex $\mathrm{q}^{\prime}$ of the second probe is contained in the $\omega$-wedge of the first probe, rooted at q. Both probes are taken along the same line $\overrightarrow{\mathrm{L}}$ (refer to Figure 11.1). Since the angle at both $\omega$-wedges is the same, at least one arm of the $\omega$-wedge at $q$ is completely outside of the interior of the $\omega$-wedge at $q^{\prime}$ and thus does not touch $\mathcal{O}$. This contradicts the definition of a valid $\omega$-probe.

LEMMA 11.4. Assume that we are given a directed line $\overrightarrow{\mathrm{L}}$. If the apex of a valid $\omega$-probe of $\mathcal{O}$ along $\overrightarrow{\mathrm{L}}$ is not a narrow vertex, then this probe is unique.

Proof. Assume to the contrary that there are two different valid $\omega$-probes of $\mathcal{O}$ along $\overrightarrow{\mathrm{L}}$ such that the apices of those probes do not touch $\mathrm{O}:\left(\mathrm{q}, \mathrm{H}_{1}, \mathrm{H}_{2}, \mathrm{p}_{1}, \mathrm{p}_{2}\right)$ and $\left(\mathrm{q}^{\prime}, \mathrm{H}_{1}^{\prime}, \mathrm{H}_{2}^{\prime}, \mathrm{p}_{1}^{\prime}, \mathrm{p}_{2}^{\prime}\right)$. By Lemma 11.3 we have $\mathrm{q}=\mathrm{q}^{\prime}$.

If $\mathrm{H}_{1} \neq \mathrm{H}_{1}^{\prime}$ (and thus $\mathrm{H}_{2} \neq \mathrm{H}_{2}^{\prime}$ ) then at least one arm of every probe does not touch $\mathcal{O}$. This is a contradiction to the validity of the probes.

If $\mathrm{q}=\mathrm{q}^{\prime}, \mathrm{H}_{1}=\mathrm{H}_{1}^{\prime}$ and $\mathrm{H}_{2}=\mathrm{H}_{2}^{\prime}$, then $\mathrm{p}_{1}=\mathrm{p}_{1}^{\prime}$ and $\mathrm{p}_{2}=\mathrm{p}_{2}^{\prime}$ according to the definition of a valid $\omega$-probe. 


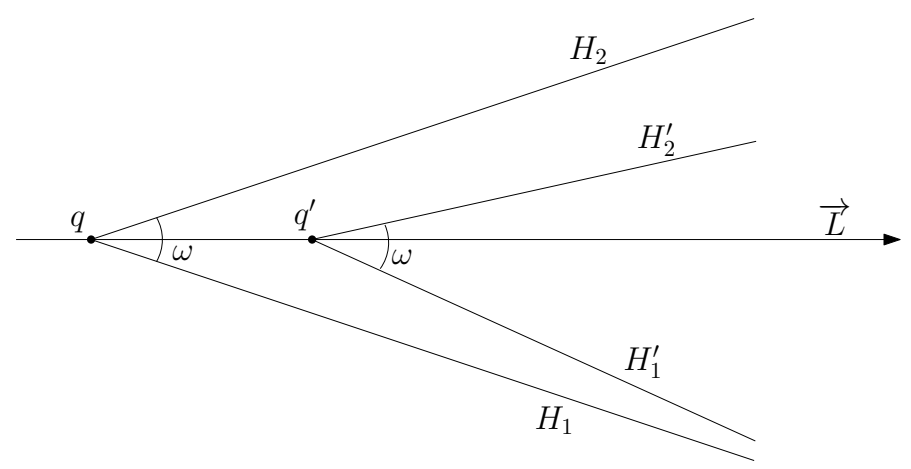

Figure 11.1: Illustration of the proof of Lemma 11.3. The arm $\mathrm{H}_{2}$ is completely outside of the interior of the $\omega$-wedge at $\mathrm{q}^{\prime}$ and thus does not touch $\mathcal{O}$.

LEMMA 11.5. Let $\mathrm{Q}$ be a convex polygon. Let e be an edge of $\mathrm{Q}$. Suppose, $\mathrm{F}_{\mathrm{Q}, \mathrm{e}}$ (see Definition 10.8) is not a subset of the line through $e$. Then, $\mathrm{F}_{\mathrm{Q}, \mathrm{e}}$ contains a triangle with base e.

In a more intuitive way, Lemma 11.5 states the following: Let $Q$ be the convex hull of the vertices of $\mathcal{O}$ that have been discovered so far, and let $e=\left(v_{1}, v_{2}\right)$ be an edge of $\mathrm{Q}$. As long as $e$ has not been confirmed to be an edge of $\mathcal{O}$, there is a possibility for an additional vertex to exist between $v_{1}$ and $v_{2}$ on the boundary of $\mathcal{O}$.

To demonstrate the difficulty of the reconstruction problem, and to present a thorough study of our probing tool, we start with polygons without narrow vertices. 


\section{POLYGONS WITHOUT NARROW VERTICES}

\section{2}

In this chapter we investigate the reconstruction of convex polygons that do not have narrow vertices. This guarantees that during the reconstruction we will not encounter any case where the apex of the wedge touches the polygon. Let $\mathcal{O}$ be a convex polygon whose exact shape and orientation are unknown. However, we are given a point $p$ and a circle $\Psi$ such that $p \in \mathcal{O} \subset \Psi$. Each probe is initialized in an orientation where it encloses $\Psi$. A probe returns the coordinates of the points of contact with the polygon $\mathcal{O}$ (as shown in Figure 10.2), the orientation of the arms and the coordinates of the apex $\mathrm{q}$.

\subsection{ALGORITHM FOR $0<\omega \leqslant \pi / 2$}

The algorithm, given below, knows that all the internal angles of $\mathcal{O}$ are larger than $\omega$. We will explain how to deal with general convex polygons in Chapter 13. Notice, that at the start of the algorithm, the algorithm does not know $n$ - the number of vertices of $\mathcal{O}$.

As part of the input the algorithm requires a polygon $Q \subseteq \mathcal{O}$, such that each vertex of $\mathrm{Q}$ is a vertex of $\mathcal{O}$ and $Q$ can contain as little as two vertices of $\mathcal{O}$. The input for the algorithm depends on the value of $\omega$. In Section 12.2 we describe a specific case when $\omega=\pi / 2$. In this case our input can be a bit more complicated ( $Q$ can be quadrilateral or pentagon), and we can reduce the number of spent probes by one. The input for the algorithm is constructed during initial probing, which can consist of one probe only (for general $\omega$ ) or three to four probes (for $\omega=\pi / 2$ ). Each vertex $u$ of the convex polygon $Q$ has a Boolean variable flag $(u)$ that is true if $u$ and its counter-clockwise successor in $Q$ form an edge of $\mathcal{O}$. The algorithm uses variable $F$ that is equal to the number of vertices of $Q$ whose flag is false. The role of the flags together with the construction of the input for the algorithm is given below. Notice, this input is suitable for any $\omega$ in the range $0<\omega \leqslant \pi / 2$.

Input 1:

\section{Invariant:}

1. $\mathrm{Q}$ is a convex polygon.

2. Each vertex of $Q$ is a vertex of $\mathcal{O}$ (and, therefore, $Q$ is contained in $\mathcal{O}$ ).

3. Each vertex $u$ of $Q$ stores a Boolean variable $\operatorname{flag}(u)$. If $\operatorname{flag}(u)=$ true, then $u$ and its counter-clockwise successor in $Q$ form an edge of $\mathcal{O}$.

4. $\mathrm{F}$ is a variable such that $\mathrm{F}=\mid\{\mathrm{u} \in \mathrm{Q}: \operatorname{flag}(\mathrm{u})=$ false $\} \mid$.

Initialization: To initialize the algorithm, choose an arbitrary line $\overrightarrow{\mathrm{L}}$ that contains $p$. Let $\left(q, H_{1}, H_{2}, p_{1}, p_{2}\right)$ be the outcome of the probe along $\vec{L}$. Initialize $Q$ as the polygon consisting of the vertices $p_{1}$ and $p_{2}$, set flag $\left(p_{1}\right)=$ false, flag $\left(p_{2}\right)=$ false, and $\mathrm{F}=2$.

Since $\mathcal{O}$ has no narrow vertices, every probe returns two distinct points of contact $p_{1}$ and $p_{2}$ that are vertices of $\mathcal{O}$. The input convex polygon $Q$ consists of already discovered vertices of $\mathcal{O}$. In every iteration, the algorithm takes two consecutive vertices of $Q, v_{1}$ and $v_{2}$, and checks whether they share an edge in $\mathcal{O}$ by shooting a probe along the line that contains $v_{1}$ and $v_{2}$. This probe either confirms an edge or, if there is no edge that connects $v_{1}$ and $v_{2}$ in $\mathcal{O}$, the probe reveals a vertex of $\mathcal{O}$ that belongs to the boundary between $v_{1}$ and $v_{2}$. This new vertex is then added to $\mathrm{Q}$. In both cases the other arm 
of the probe may also reveal new information about $\mathcal{O}$ or may return a vertex or edge of $\mathcal{O}$ that have already been discovered. The polygon $Q$ grows from within $\mathcal{O}$. When all the edges of $Q$ are confirmed, the algorithm stops, meaning that $\mathrm{Q}$ is equal to $\mathcal{O}$.

The basic algorithm for the reconstruction of the polygon without narrow vertices is given below.

\section{Algorithm (Input):}

While $\mathrm{F} \neq 0$, do the following:

1. Take an arbitrary vertex $u$ in $Q$ for which $f l a g(u)=$ false, and let $v$ be the counterclockwise successor of $u$ in $Q$.

2. Let $\vec{L}$ be the line through $u$ and $v$ that is directed from $u$ to $v$. Let $\left(q, H_{1}, H_{2}, p_{1}, p_{2}\right)$ be the outcome of the probe along $\overrightarrow{\mathrm{L}}$.

3. Case $1: p_{1} \neq u$ and $p_{2} \neq u$.

- Insert $\mathrm{p}_{1}$ into $\mathrm{Q}$ between $u$ and $v$, and set $f$ lag $\left(\mathrm{p}_{1}\right)=$ false and $\mathrm{F}=\mathrm{F}+1$.

- If $\mathrm{p}_{2}$ is not a vertex of $\mathrm{Q}$, then replace $\mathrm{Q}$ by the convex hull of $\mathrm{Q} \cup\left\{\mathrm{p}_{2}\right\}$, and set $\operatorname{flag}\left(\mathrm{p}_{2}\right)=$ false and $\mathrm{F}=\mathrm{F}+1$.

Case 2: Either $p_{1}=u$ or $p_{2}=u$.

- If $\mathrm{p}_{1}=\mathrm{u}$, then set flag $(\mathrm{u})=$ true and $\mathrm{F}=\mathrm{F}-1$; if $\mathrm{p}_{2} \notin \mathrm{Q}$, then replace $\mathrm{Q}$ by the convex hull of $\mathrm{Q} \cup\left\{\mathrm{p}_{2}\right\}$, and set $\operatorname{flag}\left(\mathrm{p}_{2}\right)=$ false and $\mathrm{F}=\mathrm{F}+1$.

- If $\mathrm{p}_{2}=u$, then set $\operatorname{flag}(v)=$ true, insert $\mathrm{p}_{1}$ into $\mathrm{Q}$ between $u$ and $v$, and set $\operatorname{flag}\left(p_{1}\right)=$ false.

In Case 1 the pair of vertices of interest $u$ and $v$ are not connected by an edge in $\mathcal{O}$ and thus neither $p_{1}=u$ nor $p_{2}=u$. In Case $2 \overline{u v}$ is an edge of $\mathcal{O}$. So, one arm of the probe coincides with $\vec{L}$. This results in either $p_{1}=u$ or $p_{2}=u$ (the latter can happen at most once and only during the first probe after the initialization). Because $\omega>0$ the case when $p_{1}=u$ and $p_{2}=u$ is impossible.

Let us analyze the performance of the algorithm on Input 1. It follows from the algorithm that the invariant is correctly maintained. The following theorem shows that the algorithm terminates.

THEOREM 12.1. Given $\omega$-wedge, with $0<\omega \leqslant \pi / 2$, and a convex polygon $\mathcal{O}$ (whose angles are strictly bigger than $\omega)$, the above algorithm reconstructs $\mathcal{O}$ by using at most $2 \mathrm{n}-2 \mathrm{\omega}$-probes.

Proof. Let $\mathrm{n}$ denote the number of vertices of $\mathcal{O}$. Consider the quantity

$$
\Phi=2|\mathrm{Q}|-\mathrm{F},
$$

where $|\mathrm{Q}|$ denotes the number of vertices of $\mathrm{Q}$. After initialization, we have $\Phi=2$. After the first iteration of the algorithm, we have $\Phi=4$. This follows from the fact that $\mathcal{O}$ has no narrow vertices. In every subsequent iteration of the algorithm, the value of $\Phi$ increases by at least one. Notice that every vertex of $Q$ is a vertex of $\mathcal{O}$. Since, at any moment, $\Phi \leqslant 2 n$, it follows that the algorithm makes at most $2 n-3$ iterations and thus, the algorithm terminates. After termination, we have $F=0$. It then follows from the invariant that $Q=\mathcal{O}$. Finally, the number of probes made by the algorithm together with the probe spent to create the input is at most $2 n-2$.

\subsection{AN IMPROVED ANALYSIS FOR $\omega=\pi / 2$}

We now show how to reconstruct $\mathcal{O}$ with one fewer probe when $\omega=\pi / 2$. The above probing algorithm with the Input 1 can be used successfully for $\omega=\pi / 2$. As we will show, in this particular case, the input for the algorithm can be improved (by a complex initialization step) to save one probe during the whole reconstruction process. We present a modification of Input 1, on which $\mathcal{O}$ can be reconstructed 
by using at most $2 n-3 \omega$-probes. As before, we assume that all internal angles of the polygon $\mathcal{O}$ are larger than $\omega=\pi / 2$. The initialization step is more complex than the initialization of Input 1 and may consist of two or three probes. The initialization step is followed by one special probe (which we call the hit probe) that returns two new pieces of information about $\mathcal{O}$ : either two newly discovered vertices or one new vertex and one new edge of $\mathcal{O}$. This is precisely where we save one probe compared to the previous technique.

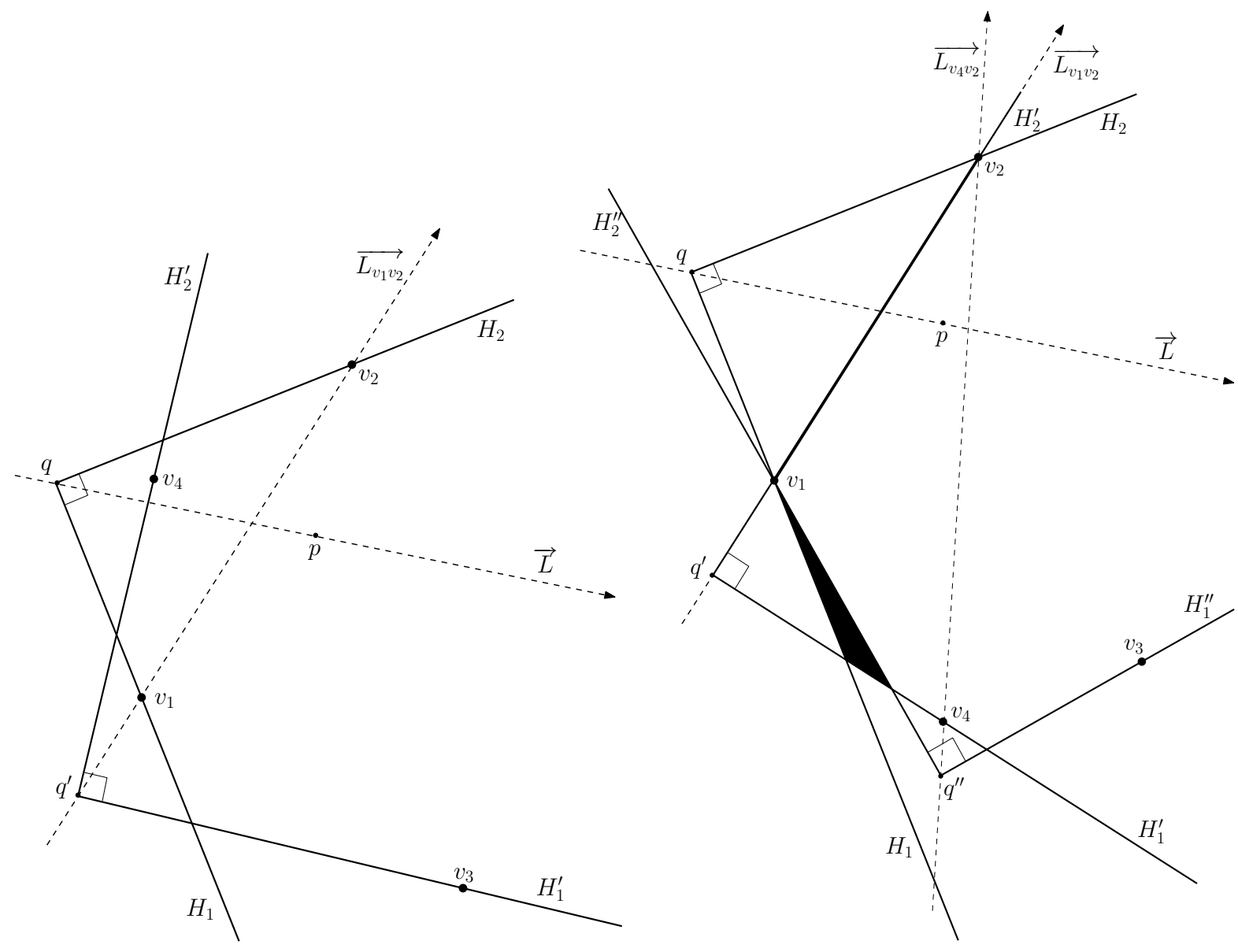

(a) First case of the initialization step.

(b) Second case of the initialization requires three probes. The figure shows $v=v_{1}$, but $v$ can be anywhere inside the black triangle.

Figure 12.1: Initialization steps of the algorithm given $\omega=\pi / 2$.

Input 2:

Invariant: Invariant of Input 1 with addition of $\omega=\pi / 2$.

Initialization: Choose an arbitrary line $\overrightarrow{\mathrm{L}}$ that contains $p$. Let $\left(\mathrm{q}, \mathrm{H}_{1}, \mathrm{H}_{2}, v_{1}, v_{2}\right)$ be the outcome of the probe along $\overrightarrow{\mathrm{L}}$ (refer to Figure 12.1). Shoot another probe along the line $\overrightarrow{\mathrm{L}_{v_{1} v_{2}}}$ (that is directed from $v_{1}$ to $v_{2}$ ). Since $\mathcal{O}$ cannot be fully contained inside the triangle $\triangle\left(q, v_{1}, v_{2}\right)$, two different outcomes of this probe are possible:

$\star$ Case 1: $\left(\mathrm{q}^{\prime}, \mathrm{H}_{1}^{\prime}, \mathrm{H}_{2}^{\prime}, v_{3}, v_{4}\right)$ - the probe returns two new vertices $v_{3}$ and $v_{4}$. Refer to Figure 12.1a. 
Initialize $\mathrm{Q}$ as the polygon consisting of the vertices $v_{1}, v_{2}, v_{3}$ and $v_{4}$, set flag $\left(v_{1}\right)=$ false, flag $\left(v_{2}\right)=$ false, flag $\left(v_{3}\right)=$ false, flag $\left(v_{4}\right)=$ false and $\mathrm{F}=4$.

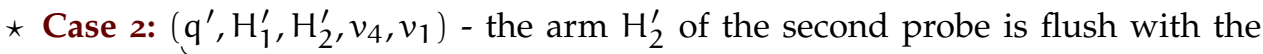
line $\overrightarrow{\mathrm{L}_{v_{1} v_{2}}}$ and thus confirms the edge $v_{1} v_{2}$ of $\mathcal{O}$. The other arm of the probe $\mathrm{H}_{1}^{\prime}$ reveals a new vertex $v_{4}$ of $\mathcal{O}$. In this case, we proceed with a third probe (refer to Figure 12.1b). We probe along the line $\overrightarrow{\mathrm{L}_{v_{4} v_{2}}}$ (directed from $v_{4}$ to $v_{2}$ ). The line segment $v_{4} v_{2}$ cannot be an edge of $\mathcal{O}$, otherwise $v_{2}$ would be a narrow vertex. So, the outcome $\left(\mathrm{q}^{\prime \prime}, \mathrm{H}_{1}^{\prime \prime}, \mathrm{H}_{2}^{\prime \prime}, v_{3}, v\right)$ of the third probe returns a new vertex $v_{3}$ of $\mathcal{O}$. The vertex $v$ can be a new vertex of $\mathcal{O}$ or it can be an already discovered vertex $v_{1}$.

Initialize $\mathrm{Q}$ as the polygon consisting of the vertices $v_{1}, v_{2}, v_{3}$ and $v_{4}$, and set $\operatorname{flag}\left(v_{1}\right)=$ false, flag $\left(v_{2}\right)=\operatorname{true}, \operatorname{flag}\left(v_{3}\right)=$ false and flag $\left(v_{4}\right)=$ false. If $v \neq v_{1}$, add $v$ to $\mathrm{Q}$, set $\operatorname{flag}(v)=$ false and $\mathrm{F}=4$. Otherwise, if $v=v_{1}$, set $\mathrm{F}=3$.

Hit Step: We choose the direction $\vec{L}$ of the hit step probe as follows:

- If the initialization step unfolded according to the first scenario (refer to Figures $12.1 \mathrm{a}$ and $12.2 \mathrm{a})$, then :

$$
\begin{aligned}
& \text { - If } \angle\left(v_{2}, v_{3}, q^{\prime}\right)<\angle\left(q, v_{2}, v_{3}\right) \text {, set } \overrightarrow{\mathrm{L}}=\overrightarrow{\mathrm{L}_{v_{3} v_{1}}} \text {. } \\
& \text { - If } \angle\left(v_{2}, v_{3}, \mathrm{q}^{\prime}\right) \geqslant \angle\left(\mathrm{q}, v_{2}, v_{3}\right) \text {, set } \overrightarrow{\mathrm{L}}=\overrightarrow{\mathrm{L}_{v_{2} v_{4}}} \text {. }
\end{aligned}
$$

- If the initialization step followed the second scenario and required three probes (refer to Figures 12.1b and 12.2b), then set $\overrightarrow{\mathrm{L}}=\overrightarrow{\mathrm{L}_{v_{3} v_{4}}}$.

Let $\left(\mathrm{q}^{*}, \mathrm{H}_{1}^{*}, \mathrm{H}_{2}^{*}, \mathrm{p}_{1}^{*}, \mathrm{p}_{2}^{*}\right)$ be the outcome of the hit step probe. Three cases are possible:

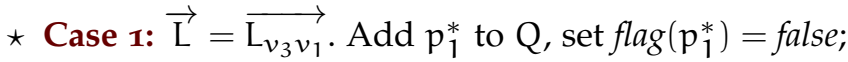

if $p_{2}^{*}=v_{3}$, then set $f \operatorname{lag}\left(v_{1}\right)=$ true;

if $p_{2}^{*} \neq v_{3}$, then add $p_{2}^{*}$ to $Q$, set $f l a g\left(p_{2}^{*}\right)=$ false and $F=6$.

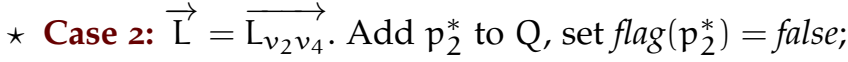

if $p_{1}^{*}=v_{2}$, then set $f \operatorname{lag}\left(v_{2}\right)=$ true;

if $p_{1}^{*} \neq v_{2}$, then add $p_{1}^{*}$ to $Q$, set $f$ lag $\left(p_{1}^{*}\right)=$ false and $F=6$.

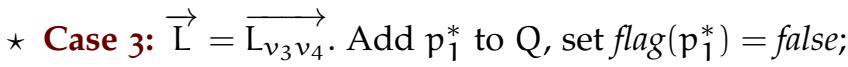

if $p_{2}^{*}=v_{3}$, then set $f \operatorname{lag}\left(v_{4}\right)=$ true;

if $\mathrm{p}_{2}^{*} \neq v_{3}$, then add $\mathrm{p}_{2}^{*}$ to $\mathrm{Q}$, set flag $\left(\mathrm{p}_{2}^{*}\right)=$ false and $\mathrm{F}=\mathrm{F}+2$.

LEMMA 12.2 (Justification of initialization steps). Given a convex polygon $\mathcal{O}$ with $\mathrm{n} \geqslant 5$ vertices and no narrow vertices; $\omega=\pi / 2$; polygon $Q=\left\{v_{1}, v_{2}, v_{3}, v_{4}\right.$, and possibly $\left.v\right\}$ of vertices of $\mathcal{O}$ discovered during the initialization step of Input 2, the line segment $v_{3} v_{2}$ is not an edge of $\mathcal{O}$.

Proof. We prove this lemma by contradiction. Let us assume that the line segment $v_{3} v_{2}$ is an edge of $\mathcal{O}$. We consider the two outcomes of the probe along the line $\overrightarrow{\mathrm{L}_{v_{1}} v_{2}}$ (the second probe of the initialization step) separately.

1. Assume that we are in the first case of the initialization (refer to Figure 12.1a). The $\omega$-wedges at $q$ and $q^{\prime}$ are valid $\omega$-probes of $\mathcal{O}$. Thus the vertices of $\mathcal{O}$ belong to the intersection of these wedges. By construction, the probe with apex $\mathrm{q}^{\prime}$ was shot along the line $\overrightarrow{\mathrm{L}_{v_{1} v_{2}}}$. Moreover, $\mathcal{O}$ has no narrow vertices. Therefore, $\mathrm{q} \neq v_{1}, \mathrm{q} \neq v_{2}, \mathrm{q}^{\prime} \neq v_{1}$ (and thus $\mathrm{q} \neq \mathrm{q}^{\prime}$ ). The line segment $\mathrm{q} \mathrm{q}^{\prime}$ is disjoint with the interiors of the $\omega$-wedges at $q$ and $q^{\prime}$, otherwise, $v_{1}=q^{\prime}$. Therefore, the angles $\angle\left(v_{3}, q^{\prime}, q\right)$ and $\angle\left(q^{\prime}, q, v_{2}\right)$ are strictly bigger than $\pi / 2$. 
Since $\mathcal{O}$ has no narrow vertices, the internal angles of $\mathcal{O}$ at $\nu_{2}$ and $\nu_{3}$ are bigger than $\pi / 2$. Since $v_{3} v_{2}$ is an edge of $\mathcal{O}$, the angles $\angle\left(q, v_{2}, v_{3}\right)$ and $\angle\left(v_{2}, v_{3}, \mathbf{q}^{\prime}\right)$ are strictly bigger than $\pi / 2$. Consider the quadrilateral $\left\{\mathbf{q}, \mathbf{q}^{\prime}, v_{3}, v_{2}\right\}$. All its internal angles are bigger than $\pi / 2$, which is a contradiction. Therefore, $v_{3} v_{2}$ is not an edge of $\mathcal{O}$.

2. Assume that we are in the second case of the initialization (refer to Figure 12.1b). Note that, in this case, the arm $\mathrm{H}_{2}^{\prime \prime}$ of the third probe (with apex $\mathrm{q}^{\prime \prime}$ ) contains the vertex $v$ of $\mathcal{O}$, that may or may not be equal to $v_{1}$. The vertex $v$ belongs to the intersection of the $\omega$-wedges at $q$ and $\mathrm{q}^{\prime}$ and is to the left of the line $\overrightarrow{\mathrm{L}_{\mathrm{q}^{\prime \prime} v_{1}}}$ or on it. The region where $v$ belongs appears in black in Figure $12.1 \mathrm{~b}$; the arm $\mathrm{H}_{2}^{\prime \prime}$ intersects this area. Because $\mathcal{O}$ has no narrow vertices, $v_{1} \neq \mathrm{q}, v_{1} \neq \mathrm{q}^{\prime}$ and $v_{4} \neq \mathrm{q}^{\prime \prime}$. Therefore, the line segment $\mathrm{q}^{\prime} \mathrm{q}^{\prime \prime}$ is disjoint with the interiors of the $\omega$-wedges at $\mathrm{q}^{\prime}$ and $\mathrm{q}^{\prime \prime}$. Thus, the angles $\angle\left(v_{3}, \mathrm{q}^{\prime \prime}, \mathrm{q}^{\prime}\right)$ and $\angle\left(\mathrm{q}^{\prime \prime}, \mathrm{q}^{\prime}, v_{2}\right)$ are strictly bigger than $\pi / 2$.

Since $\mathcal{O}$ has no narrow vertices and $v_{1} v_{2}$ is an edge of $\mathcal{O}$, the angles $\angle\left(\mathrm{q}^{\prime}, v_{2}, v_{3}\right)$ and $\angle\left(v_{2}, v_{3}, \mathrm{q}^{\prime \prime}\right)$ are strictly bigger than $\pi / 2$. Consider the quadrilateral $\left\{q^{\prime}, q^{\prime \prime}, v_{3}, v_{2}\right\}$. All its internal angles are bigger than $\pi / 2$, which is a contradiction. Therefore, $v_{3} v_{2}$ cannot be an edge of $\mathcal{O}$.

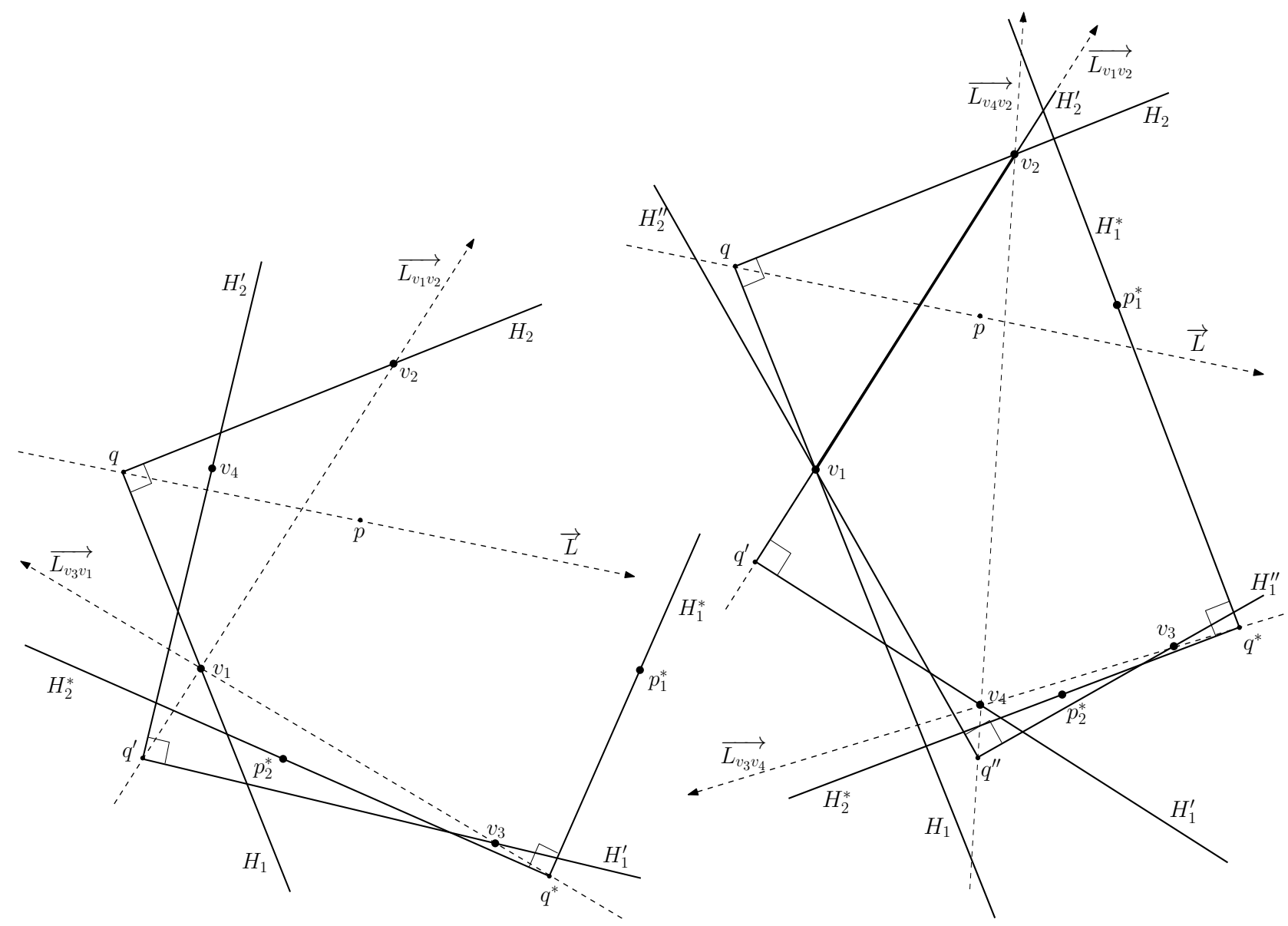

(a) Hit Step after the first case of the initialization. (b) Hit Step after the second case of the initialization. The vertex $v$ is shown to be equal to the vertex $v_{1}$.

Figure 12.2: Possible outcomes of the Hit Step of the Input 2. 
LEMMA 12.3 (Hit Step justification). Given a convex polygon $\mathcal{O}$ with $\mathrm{n} \geqslant 5$ vertices and no narrow vertices; $\omega=\pi / 2$; polygon $\mathrm{Q}=\left\{v_{1}, v_{2}, v_{3}, v_{4}\right.$, and possibly $\left.v\right\}$, of vertices of $\mathcal{O}$ discovered during the initialization step of Input 2. The Hit Step of Input 2 (that consists of one probe only), results in two new pieces of information: either two newly discovered vertices of $\mathcal{O}$, or one newly discovered vertex and one newly confirmed edge of $\mathcal{O}$.

Proof. The Hit Step of Input 2 consists of one probe only. We choose the direction of this probe to be one of the following two:

1. $\overrightarrow{\mathrm{L}_{v_{3} x_{1}}}$, where $x_{1}$ is a clockwise neighbour of $v_{3}$ in $\mathrm{Q}$.

2. $\overrightarrow{\mathrm{L}_{v_{2} x_{2}}}$, where $x_{2}$ is a counter-clockwise neighbour of $v_{2}$ in $\mathrm{Q}$ (refer to Figure 12.1).

There are two possible outcomes of the probe along the line $\overrightarrow{\mathrm{L}_{v_{1} v_{2}}}$ (the second probe of the initialization step of Input 2). We consider those cases separately.

- Case 1: Assume, that the probe along $\overrightarrow{\mathrm{L}_{v_{1} v_{2}}}$ returns two new vertices $v_{3}$ and $v_{4}$ (refer to Figure 12.1a). We showed in the proof of Lemma 12.2, that the angles $\angle\left(v_{3}, \mathbf{q}^{\prime}, \mathrm{q}\right)$ and $\angle\left(\mathrm{q}^{\prime}, \mathrm{q}, v_{2}\right)$ are strictly bigger than $\pi / 2$. Thus, in the quadrilateral $\left\{\mathbf{q}, \mathbf{q}^{\prime}, v_{3}, v_{2}\right\}$ at least one of the angles $\angle\left(v_{2}, v_{3}, q^{\prime}\right)$ or $\angle\left(q, v_{2}, v_{3}\right)$ is strictly smaller than $\pi / 2$. Consider the smallest one, which is smaller than $\pi / 2$. If $\angle\left(v_{2}, v_{3}, \mathrm{q}^{\prime}\right)<\angle\left(\mathrm{q}, v_{2}, v_{3}\right)$, then we choose $\overrightarrow{\mathrm{L}_{v_{3}} v_{1}}$ as a direction for the Hit Step probe. Otherwise, we choose $\overrightarrow{\mathrm{L}_{v_{2} v_{4}}}$.

We focus on the case where $\angle\left(v_{2}, v_{3}, \mathrm{q}^{\prime}\right)<\angle\left(\mathrm{q}, v_{2}, v_{3}\right)$. The other case is similar. We shoot our probe along the line $\overrightarrow{\mathrm{L}_{v_{3} v_{1}}}$. Let $\left(\mathrm{q}^{*}, \mathrm{H}_{1}^{*}, \mathrm{H}_{2}^{*}, \mathrm{p}_{1}^{*}, \mathrm{p}_{2}^{*}\right)$ be the outcome of the probe. Refer to Figure 12.2a. Since $v_{3}$ is not a narrow vertex, $\mathrm{q}^{*} \neq v_{3}$. Similarly to what was shown in the proof of Lemma 12.2, the line segment $\mathrm{q}^{\prime} \mathrm{q}^{*}$ is disjoint with the interiors of the $\omega$-wedges at $\mathrm{q}^{\prime}$ and $\mathrm{q}^{*}$, leading to the conclusion that the angles $\angle\left(\mathrm{p}_{1}^{*}, \mathrm{q}^{*}, \mathrm{q}^{\prime}\right)$ and $\angle\left(\mathrm{q}^{*}, \mathrm{q}^{\prime}, v_{4}\right)$ are strictly bigger than $\pi / 2$. The arm $\mathrm{H}_{1}^{*}$ contains the vertex $p_{1}^{*}$ of $\mathcal{O}$ and it is to the right of the line $\overrightarrow{\mathrm{L}_{v_{3} v_{1}}}$ (refer to Definition 10.3). The vertex $p_{1}^{*}$ satisfies the following properties:

1. $p_{1}^{*} \neq v_{3}$. Otherwise, the vertices $v_{2}$ and $v_{4}$ would not belong to the interior of the wedge at $\mathrm{q}^{*}$, which is a contradiction to the validity of the probe. Similarly, $\mathrm{p}_{1}^{*} \neq v_{1}$.

2. $p_{1}^{*} \neq v_{2}$. We showed previously, that $\angle\left(p_{1}^{*}, q^{*}, q^{\prime}\right)>\pi / 2$. Assume, to the contrary, that $\mathrm{p}_{1}^{*}=v_{2}$. Thus, $\angle\left(v_{2}, \mathrm{q}^{*}, \mathrm{q}^{\prime}\right)>\pi / 2$. The vertex $v_{3}$ is inside the wedge induced by $\mathrm{q}^{\prime}$, $\mathrm{q}^{*}$ and $v_{2}$ according to the construction. Therefore, the angle $\angle\left(v_{2}, v_{3}, \mathrm{q}^{\prime}\right)$ is bigger than $\angle\left(v_{2}, \mathrm{q}^{*}, \mathrm{q}^{\prime}\right)$. This is a contradiction because $\angle\left(v_{2}, v_{3}, \mathrm{q}^{\prime}\right)<\pi / 2$.

3. $p_{1}^{*} \neq v_{4}$. Otherwise, the line segment $q^{\prime} q^{*}$ would be completely inside the interior of the $\omega$-wedges at $\mathrm{q}^{\prime}$ and $\mathrm{q}^{*}$, which is impossible according to the construction.

Consequently, $p_{1}^{*}$ is a newly discovered vertex of $\mathcal{O}$.

The other arm $\mathrm{H}_{2}^{*}$ of the probe contains the vertex $p_{2}^{*}$ of $\mathcal{O}$ and it is to the left of the line $\overrightarrow{\mathrm{L}_{v_{3}} v_{1}}$ or on it (by Definition 10.3). If $p_{2}^{*} \in \overrightarrow{\mathrm{L}_{v_{3} v_{1}}}$ then the arm $\mathrm{H}_{2}^{*}$ coincides with the line $\overrightarrow{\mathrm{L}_{v_{3} v_{1}}}$ and as a result the edge $v_{1} v_{3}$ of $\mathcal{O}$ is confirmed. If $p_{2}^{*} \notin \overrightarrow{L_{v_{3}} v_{1}}$ then $p_{2}^{*}$ must be a newly discovered vertex of $\mathcal{O}$, since there were no vertices of $\mathcal{O}$ discovered so far to the left of $\overrightarrow{\mathrm{L}_{v_{3} v_{1}}}$. In either way, the arm $\mathrm{H}_{2}^{*}$ reveals new information about $\mathcal{O}$.

We conclude, that the probe of the Hit Step, that follows the first case of the initialization step, contributes two new pieces of information.

- Case 2: Assume, that the probe along $\overrightarrow{\mathrm{L}_{v_{1} v_{2}}}$ confirms the edge $v_{1} v_{2}$ of $\mathcal{O}$ (refer to Figure 12.1b). In this case the direction for the Hit Step probe will be $\overrightarrow{\mathrm{L}_{v_{3} v_{4}}}$ (refer to Figure 12.2b). Let $\left(\mathrm{q}^{*}, \mathrm{H}_{1}^{*}\right.$, $\mathrm{H}_{2}^{*}, \mathrm{p}_{1}^{*}, \mathrm{p}_{2}^{*}$ ) be the outcome of this probe. Similarly to the previous case, we can show that the line segment $q^{\prime \prime} q^{*}$ is disjoint with the interiors of the $\omega$-wedges at $q^{\prime \prime}$ and $q^{*}$, leading to the 
following conclusion: $\angle\left(\mathrm{p}_{1}^{*}, \mathrm{q}^{*}, \mathrm{q}^{\prime \prime}\right) \geqslant \pi / 2$ and $\angle\left(\mathrm{q}^{*}, \mathrm{q}^{\prime \prime}, v\right) \geqslant \pi / 2$. Note, that in Figure 12.2b, the vertex $v$ is shown to be equal to the vertex $v_{1}$. Let $t$ be the intersection point between $\mathrm{H}_{2}^{\prime}$ and $\mathrm{H}_{2}^{\prime \prime}$. Note, that if $v=v_{1}$, then $\mathrm{t}=v_{1}$. The angle $\angle\left(\mathrm{q}^{\prime \prime}, \mathrm{t}, v_{2}\right)>\pi / 2$, otherwise $\mathrm{q}^{\prime \prime}$ would be inside the $\omega$-wedge at $q^{\prime}$ together with the line segment $q^{\prime} q^{\prime \prime}$, which is a contradiction (refer to the proof of the Lemma 12.2).

The arm $\mathrm{H}_{1}^{*}$ contains the vertex $p_{1}^{*}$ of $\mathcal{O}$ and it is to the right of $\overrightarrow{\mathrm{L}_{v_{3} v_{4}}}$ (refer to Definition 10.3). The vertex $p_{1}^{*}$ satisfies the following properties:

1. $p_{1}^{*} \neq v_{3}$. Otherwise, the vertices $v_{2}, v_{1}$ and $v$ would not belong to the interior of the wedge at $\mathrm{q}^{*}$, which is a contradiction to the validity of the probe. Similarly, $\mathrm{p}_{1}^{*} \neq v_{4}$.

2. $p_{1}^{*} \neq v_{2}$. Assume, to the contrary, that $p_{1}^{*}=v_{2}$. Consider the quadrilateral $\left\{q^{\prime \prime}, q^{*}, v_{2}, t\right\}$. We showed before, that $\angle\left(v_{2}, \mathrm{q}^{*}, \mathrm{q}^{\prime \prime}\right)=\angle\left(\mathrm{p}_{1}^{*}, \mathrm{q}^{*}, \mathrm{q}^{\prime \prime}\right) \geqslant \pi / 2, \angle\left(\mathrm{q}^{*}, \mathrm{q}^{\prime \prime}, \mathrm{t}\right)=\angle\left(\mathrm{q}^{*}, \mathrm{q}^{\prime \prime}, v\right) \geqslant$ $\pi / 2$ and $\angle\left(\mathrm{q}^{\prime \prime}, \mathrm{t}, v_{2}\right)>\pi / 2$. This implies that the fourth internal angle $\angle\left(\mathrm{t}, v_{2}, \mathrm{q}^{*}\right)=$ $\angle\left(v_{1}, v_{2}, \mathrm{q}^{*}\right)$ of the quadrilateral must be smaller than $\pi / 2$. All the probes made so far are valid $\omega$-probes of $\mathcal{O}$ and thus $\mathcal{O}$ should reside inside the intersection of all $\omega$-wedges. Thus, the internal angle of $\mathcal{O}$ at $v_{2}$ is inside the wedge induced by $\mathrm{q}^{*}, v_{2}$ and $v_{1}$ and therefore it is smaller than $\pi / 2$. This is a contradiction, because $v_{2}$ is not a narrow vertex.

3. $p_{1}^{*} \neq v$. Otherwise, the line segment $q^{\prime \prime} q^{*}$ is completely inside the interiors of the $w$ wedges at $\mathrm{q}^{\prime \prime}$ and $\mathrm{q}^{*}$, which is impossible according to the construction.

4. $p_{1}^{*} \neq v_{1}$. The $\omega$-wedge at $q^{*}$ is a valid $\omega$-probe and thus must contain the edge $v_{1} v_{2}$. In order for the arm $\mathrm{H}_{1}^{*}$ to contain the vertex $v_{1}$, the apex of the probe $\mathrm{q}^{*}$ should be to the left of $\overrightarrow{\mathrm{L}_{v_{1} v_{2}}}$, which is impossible according to the construction.

Consequently, $\mathrm{p}_{1}^{*}$ is a newly discovered vertex of $\mathcal{O}$. Similarly to the previous case, the arm $\mathrm{H}_{2}^{*}$ reveals new information about $\mathcal{O}$ (it may be an edge or a vertex). We showed that the probe of the Hit Step, that follows the second case of the initialization step, contributes two new pieces of information.

Let us analyze the performance of the algorithm on Input 2. It follows from the algorithm that the invariant is correctly maintained. The following theorem shows that the algorithm terminates.

THEOREM 12.4. Given $\omega$-wedge such that $\omega=\pi / 2$ and a convex polygon $\mathcal{O}$ (whose angles are strictly bigger than $\omega)$, the algorithm on Input 2 reconstructs $\mathcal{O}$ using at most $2 \mathrm{n}-3 \mathrm{\omega}$-probes.

Proof. Let $\mathrm{n}$ denote the number of vertices of $\mathcal{O}$. Consider the quantity

$$
\Phi=2|\mathrm{Q}|-\mathrm{F},
$$

where $|Q|$ denotes the number of vertices of $Q$. At the start of the algorithm, we have $\Phi=2$. At the end of the initialization step, if we are in the first case (the probe along $\overrightarrow{\mathrm{L}_{v_{1} v_{2}}}$ returns two new vertices), then $|\mathrm{Q}|=4, \mathrm{~F}=4$ and thus $\Phi=4$. Otherwise,

- if $v=v_{1}$, then $|\mathrm{Q}|=4, \mathrm{~F}=3$ and thus $\Phi=5$.

- if $v \neq v_{1}$, then $|\mathrm{Q}|=5, \mathrm{~F}=4$ and thus $\Phi=6$.

The Hit Step increases $\Phi$ by two. The hit probe either discovers two new vertices (and thus $|\mathrm{Q}|$ and $\mathrm{F}$ increase by two), or one new vertex and one new edge $(|\mathrm{Q}|$ increases by one and $\mathrm{F}$ does not change). In every iteration of the algorithm, the value of $\Phi$ increases by at least one. Notice, that every vertex of $Q$ is a vertex of $\mathcal{O}$. Since, at any moment, $\Phi \leqslant 2 n$, it follows that the algorithm makes at most

- $2 n-6$ iterations, if the initialization unfolded according to the first case, and 
- $2 n-7$ iterations, if the initialization followed the second case (at most $2 n-7$ iterations if $v=v_{1}$, and at most $2 n-8$ iterations if $v \neq v_{1}$ ).

Thus, the algorithm terminates. After termination, we have $F=0$. It then follows from the invariant that, at that moment, $Q$ is equal to $\mathcal{O}$. Finally, the number of probes made by the overall reconstruction process is at most $2 n-3$.

\subsection{LOWER BOUND}

In this section, we prove a lower bound on the number of probes required to reconstruct an n-gon that is equal to the number of probes used by our algorithm (refer to Theorem 12.1 and 12.4) in the worst case. We present an adversarial argument that forces any probing strategy to make a given number of probes to determine the exact shape and orientation of $\mathcal{O}$. Our goal is to show that in order to be correct, any algorithm must confirm every edge and probe for every vertex of $\mathcal{O}$. Given any sequence of probes by an algorithm, we show that the adversary can force the algorithm to spend at least $2 n-2$ probes for any $\omega$ in the range $0<\omega<\pi / 2$, and at least $2 n-3$ probes for $\omega=\pi / 2$. We outline the adversary's strategy below.

Consider an algorithm that wants to reconstruct an object $\mathcal{O}$ that is "hidden" by the adversary. The algorithm provides a direction of its next probe to the adversary. If the direction is valid, the adversary returns an output that is coherent with the information already given to the algorithm. This output consists of: two points of contact with $\mathcal{O}$, the orientation of both arms and the coordinates of the apex. For a probe that is not valid, the adversary replies that the probe missed the object and gives no additional information. The strategy of the adversary is to answer each probe by revealing new information only if it is unavoidable. We showed in Lemma 11.5 that as long as an edge $e$ of $\mathrm{Q} \subseteq \mathcal{O}$ is not confirmed (by a probe directed through that edge), the feasible region of $\mathrm{F}_{\mathrm{Q}, e}$ is not empty and does not degenerate into a line segment, meaning that the adversary has infinitely many points inside that region where it can place a vertex to deny the existence of the edge $e$. Similarly, when the algorithm aims for some place where, according to its strategy, a vertex of $\mathcal{O}$ is most likely to be hidden, the adversary orients the arms of the probe to "shrink" the feasible region of that place. The probe then reveals only known vertices and, possibly confirms an edge. For directions that do not intersect $\mathrm{Q}$, the adversary lets the probe miss the object completely. While in some cases it is unavoidable for the adversary to reveal new information about $\mathcal{O}$, it is always possible to reveal only one new piece of information per probe (except for the first two valid probes).

We show that for every algorithm, there is a convex polygon, such that, except for the first two probes, in each probe the algorithm either discovers a new vertex or verifies an edge or does not discover any new information. So, at least $2 n-2 \omega$-probes are necessary to determine a convex $n$-gon when $0<\omega<\pi / 2$. When $\omega=\pi / 2$, the adversary forces at least $2 n-3 \omega$-probes.

We assume that the algorithm is deterministic and does not repeat the same probe during one probing session.

The adversary begins by defining a circle $\Psi$ (where the polygon $\mathcal{O}$ will reside), a point $\mathrm{p}$ as the center of $\Psi$ and chooses $n \geqslant 4(n \geqslant 5$ when $\omega=\pi / 2)$ to avoid narrow vertices. The adversary maintains a closed convex curve $A$ that is initially the circle $\Psi$. During queries the adversary will change the shape of the curve $A$ such that $A$ is the boundary of the intersection of all $\omega$-probes made so far with $\Psi$. Once the adversary reveals a vertex of the polygon $\mathcal{O}$, it remains fixed on $A$, defining sections of the curve that cannot be changed. So, at the end of a query session the curve $A$ becomes the convex polygon $\mathcal{O}$.

Let $\overrightarrow{L_{x}}$ be a direction of the probe, such that $\overrightarrow{L_{x}}$ does not intersect $\mathcal{O}$. The probe along this line is considered to be non-valid (refer to the Definition 10.3) and reported as such to the algorithm. But, if $\overrightarrow{L_{x}}$ intersects $A$, we update $A$ to be the intersection of $A$ and the half-plane, that contains $\overrightarrow{L_{x}}$ on its boundary and the point $p$. 
Notice that, during the construction of the polygon, the adversary avoids to locate vertices at positions that allow existence of two edges, whose extensions form an angle of $\omega$ (refer to Figure 10.2b). Let $Q$ be the convex hull of the vertices of $\mathcal{O}$ that have been discovered, and let $e=\left(v_{1}, v_{2}\right)$ be an edge of $Q$ but not an edge of $\mathcal{O}$ (meaning that the interior of $e$ is not on the boundary of $A$ and thus $\mathrm{F}_{\mathrm{Q}, e} \backslash l_{e} \neq \emptyset$, where $\mathrm{F}_{\mathrm{Q}, e}$ is the feasible region (see Definition 10.8) and $l_{e}$ is the line that contains $e$ ). To create a new vertex $v$ between $v_{1}$ and $v_{2}$ on the boundary of $\mathcal{O}$, the adversary chooses the position for $v$ in $\mathrm{F}_{\mathrm{Q}, e} \backslash l_{e}$, such that for every edge $e_{\mathrm{Q}}$ of $\mathrm{Q}$ (but not $e$ ): $\angle\left(l_{e_{\mathrm{Q}}}, l_{v_{1}, v}\right) \neq \omega$ and $\angle\left(l_{e_{\mathrm{Q}}}, l_{v_{2}, v}\right) \neq \omega$ (where $l_{e_{\mathrm{Q}}}$ is the line that contains $e_{\mathrm{Q}}$ and $l_{v_{1}, v}$ (respectively $l_{v_{2}, v}$ ) is the line that contains $v_{1}$ and $v$ (respectively $v_{2}$ and $v$ )). It is always possible because $\mathrm{Q}$ has a finite number of edges (this number is smaller than $n$ ) and there are infinitely many positions for $v$ inside $F_{Q, e} \backslash l_{e}$. Refer to Lemma 11.5.

The strategy of the adversary can be described by four different stages. The initial stage lasts until the algorithm discovers four distinct vertices $v_{1}, v_{2}, v_{3}$ and $v_{4}$ of $\mathcal{O}$. We assumed at the beginning that $\mathcal{O}$ has no narrow vertices. This means that all the internal angles of the polygon $\mathcal{O}$ should be larger than $\omega$. To comply with this assumption, the adversary has to place $v_{1}, v_{2}, v_{3}$ and $v_{4}$ in such a way that there exists a convex polygon $\mathcal{O}$ whose vertices include $v_{1}, v_{2}, v_{3}$ and $v_{4}$, that has no internal angle smaller or equal to $\omega$ (refer to the Figure 12.3). Any algorithm will require at least two probes to discover the vertices $v_{1}, v_{2}, v_{3}$ and $v_{4}$.

Assume that the direction of the first valid probe $\vec{L}$ made by the algorithm contains $p$. Otherwise, the adversary can make an object small enough for the probe to miss the object. (If there were nonvalid probes that intersected $A$, then the adversary would create a circle inside $A$ with center $p$ and restricts its working space to this circle. For simplicity, we call this circle $\Psi$ again and update $A$ to be equal to $\Psi$.) The adversary stops the probe at any moment after the apex $q$ of the probe enters the interior of $\Psi$, but before it reaches $p$. Let $\left(q, \mathrm{H}_{1}, \mathrm{H}_{2}, \mathrm{p}_{1}, \mathrm{p}_{2}\right)$ be the outcome of the probe along $\overrightarrow{\mathrm{L}}$ returned by the adversary, such that $\mathrm{H}_{1}$ (respectively $\mathrm{H}_{2}$ ) makes a negative (respectively positive) angle of $\omega / 2$ with $\vec{L}$ (refer to Figure 12.3).

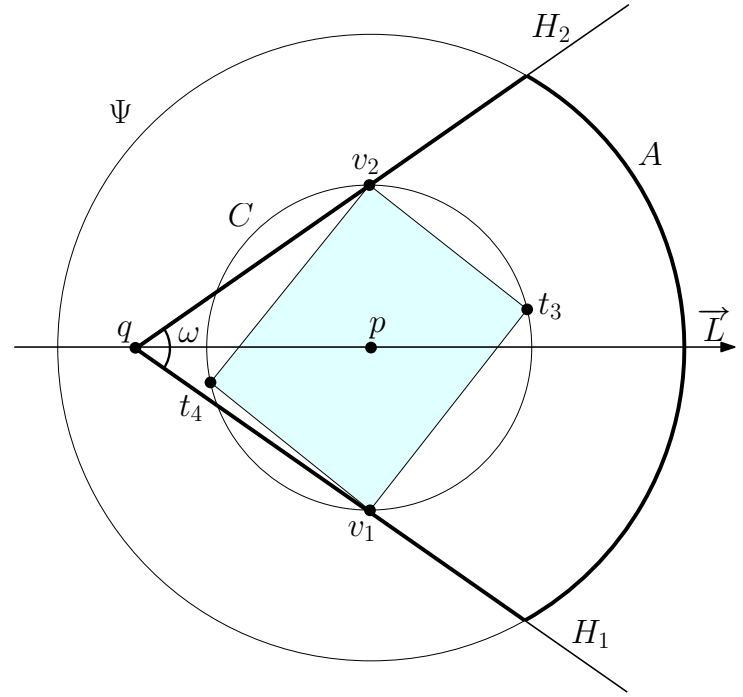

(a) Initial steps of adversary's strategy for $0<\omega<\pi / 2$

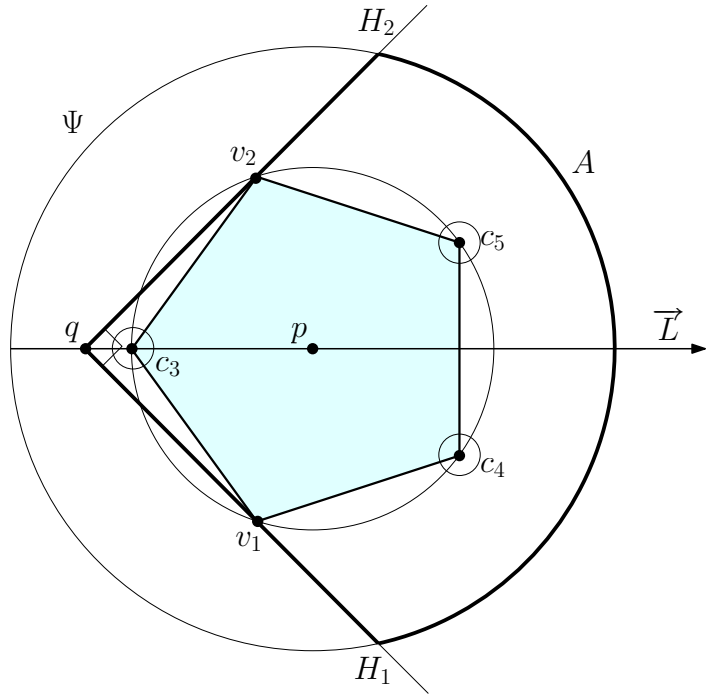

(b) Initial steps of the adversary, given $\omega=$ $\pi / 2$.

Figure 12.3: Initialization. After the first valid $\omega$-probe, the points $v_{1}$ and $v_{2}$ are fixed. The convex curve $A$ is shown in bold.

For now, assume that $0<\omega<\pi / 2$. Given $\overrightarrow{\mathrm{L}}$, we define an $\omega$-wedge in such a way that there exists a rectangle $\left\{v_{1}, t_{3}, v_{2}, t_{4}\right\}$ in the interior of the $\omega$-wedge such that it is inscribed into a circle $C$ with the center at $p$ and vertices $v_{1}$ and $v_{2}$ belong to the arms of the probe (without loss of generality assume 
that $v_{1} \in \mathrm{H}_{1}$ and $v_{2} \in \mathrm{H}_{2}$ ). The point $\mathrm{p}$ is the midpoint of the line-segment $v_{1} v_{2}$ (refer to Figure 12.3a). The adversary returns $p_{1}=v_{1}$ and $p_{2}=v_{2}$ as points of contact.

The vertices $v_{1}$ and $v_{2}$ of the rectangle are fixed. The vertices $t_{3}$ and $t_{4}$ are not fixed yet. They can be anywhere in the interior of $A$ (excluding boundary), as long as all the internal angles of the quadrilateral $\left\{v_{1}, t_{3}, v_{2}, t_{4}\right\}$ are equal to $\pi / 2$. Thus, the vertices $t_{3}$ and $t_{4}$ have temporary positions. If the direction of the next probe contains any of the four edges of the rectangle $\left\{v_{1}, t_{3}, v_{2}, t_{4}\right\}$, the points $t_{3}$ and $t_{4}$ move to avoid confirming an edge. If the probe reveals $t_{3}$ (respectively $t_{4}$ ), it becomes fixed and we rename it into $v_{3}$ (respectively $v_{4}$ ). If the probe reveals only one temporary vertex $t_{3}$ or $t_{4}$, then we only rename the revealed vertex, but fix both vertices. Then the probe outputs only the vertices it found (in this case the output will include vertex $v_{1}$ or $v_{2}$ already known by the algorithm) leaving the fourth, not revealed vertex of the rectangle, fixed by the adversary but still hidden from the algorithm. The algorithm, of course, can guess its position now. This concludes the initialization step for $0<\omega<\pi / 2$.

Let us explore the case $\omega=\pi / 2$. Given $\overrightarrow{\mathrm{L}}$, we define an $\omega$-wedge in such a way that there exists a regular pentagon $\left\{v_{1}, c_{4}, c_{5}, v_{2}, c_{3}\right\}$ in the interior of the $\omega$-wedge such that it is inscribed into a circle with center at $p$ and exactly two non-adjacent vertices $v_{1}$ and $v_{2}$ belong to the arms of the probe (without loss of generality assume that $v_{1} \in \mathrm{H}_{1}$ and $v_{2} \in \mathrm{H}_{2}$ ) (refer to Figure 12.3b). The adversary returns $p_{1}=v_{1}$ and $p_{2}=v_{2}$ as points of contact.

The vertices $v_{1}$ and $v_{2}$ of the pentagon are fixed. As in the previous case, the other three vertices can move (within some constraints) to avoid edge confirmation during probing. We create a circle $\mathrm{C}_{3}$ (respectively, $\mathrm{C}_{4}, \mathrm{C}_{5}$ ) with center $\mathrm{c}_{3}$ (respectively, $\left.\mathrm{c}_{4}, \mathrm{c}_{5}\right)$ and $\operatorname{diameter} \frac{1}{2 \sqrt{2}} \operatorname{dist}\left(v_{1}, v_{2}\right)\left(1-\tan \frac{\pi}{5}\right)$, where $\operatorname{dist}\left(v_{1}, v_{2}\right)$ is the distance between $v_{1}$ and $v_{2}$. The vertex $v_{3}$ (respectively, $\left.v_{4}, v_{5}\right)$ is fixed during later probes of the algorithm and stays in the interior of $C_{3}$ (respectively, $C_{4}, C_{5}$ ). For any point $p_{3}^{\prime}$ (respectively, $p_{4}^{\prime}, p_{5}^{\prime}$ ) within the interior of $C_{3}$ (respectively, $C_{4}, C_{5}$ ) all the interior angles of the pentagon $\left\{v_{1}, p_{4}^{\prime}, p_{5}^{\prime}, v_{2}, p_{3}^{\prime}\right\}$ are strictly bigger than $\frac{\pi}{2}$, meaning that no narrow vertex can be created. None of the circles degenerates into a point since their diameter is strictly bigger than zero.

The adversary maintains an $\omega$-cloud $\Omega$ of the pentagon $\left\{v_{1}, c_{4}, c_{5}, v_{2}, c_{3}\right\}$. If, for example, the direction of the probe enters the cloud through the ark that is supported by $c_{3}$ and $c_{4}$, then the adversary fixes two new vertices $v_{3}$ and $v_{4}$, such that $v_{3}=c_{3}$ and $v_{4}=c_{4}$. Every query of the algorithm will be answered by the adversary (until the algorithm discovers two new vertices) considering $\Omega$, unless the apex of the probe coincides with the pivot point on the cloud (refer to Definition 10.5), meaning that the direction of the probe coincides with one of the edges of the pentagon. Several cases are possible in this situation:

1. Assume that only $v_{1}$ and $v_{2}$ are fixed and that the vertices $v_{3}, v_{4}$ and $v_{5}$ are unknown. There are several cases to consider. The direction of the probe $\overrightarrow{\mathrm{L}}$ may contain:

- $\overrightarrow{v_{1} \mathrm{C}_{3}}$ (respectively, $\overrightarrow{v_{1} \mathrm{C}_{4}}$ ) - choose two vertices $v_{3}$ and $v_{4}$, such that $v_{3} \in \mathrm{C}_{3}, v_{4} \in \mathrm{C}_{4} ; v_{3}$ and $v_{4}$ are in the interior of $A$ and $v_{3} \notin \overrightarrow{\mathrm{L}}$ (respectively, $v_{4} \notin \overrightarrow{\mathrm{L}}$ ). Delete $\mathrm{c}_{3}, \mathrm{C}_{3}, \mathrm{c}_{4}$ and $\mathrm{C}_{4}$, and update $\Omega$. As the two points of contact return $v_{1}$ and $v_{3}$ (respectively, $v_{1}$ and $v_{4}$ ).

- $\overrightarrow{v_{2} \mathrm{c}_{3}}$ (respectively, $\overrightarrow{v_{2} \mathrm{c}_{5}}$ ) - similar to the previous case.

- $\overrightarrow{c_{3} v_{1}}$ (respectively, $\overrightarrow{c_{3} v_{2}}$ ) - choose a vertex $v_{3}$ furthest from the segment $v_{1} v_{2}$, such that $v_{3} \in C_{3}, v_{3}$ is in the interior of $A$ and $v_{3} \notin \overrightarrow{\mathrm{L}}$. Delete $c_{3}$ and $C_{3}$, and update $\Omega$. Return $v_{3}$ and $v_{1}$ (respectively, $v_{3}$ and $v_{2}$ ) as the two points of contact.

- $\overrightarrow{\mathrm{c}_{4} \mathrm{c}_{5}}$ - choose a vertex $v_{4}$ furthest from the segment $v_{1} \mathrm{c}_{5}$, such that $v_{4} \in \mathrm{C}_{4}, v_{4}$ is in the interior of $A$ and $v_{4} \notin \overrightarrow{\mathrm{L}}$. Delete $\mathrm{c}_{4}$ and $\mathrm{C}_{4}$, and update $\Omega$. Return $v_{4}$ and $v_{1}$ as the two points of contact.

- $\overrightarrow{\mathrm{c}_{5} \mathrm{c}_{4}}$ - similar to the previous case. 
- $\overrightarrow{\mathrm{c}_{4} v_{1}}$ (respectively, $\overrightarrow{\mathrm{c}_{5} v_{2}}$ ) - choose two vertices $v_{4}$ and $v_{5}$, such that $v_{4} \in \mathrm{C}_{4}, v_{5} \in \mathrm{C}_{5}, v_{4}$ and $v_{5}$ are in the interior of $A$ and $v_{4} \notin \overrightarrow{\mathrm{L}}$ (respectively, $v_{5} \notin \overrightarrow{\mathrm{L}}$ ). Delete $\mathrm{c}_{4}, \mathrm{C}_{4}, \mathrm{c}_{5}$ and $\mathrm{C}_{5}$, and update $\Omega$. Return $v_{1}$ and $v_{4}$ (respectively, $v_{2}$ and $v_{5}$ ) as the two points of contact.

2. If $\vec{L}$ contains two fixed vertices, meaning, that at least three vertices of the pentagon are known to the algorithm, then the adversary proceeds similarly to the second step of its protocol (read further) by revealing a new vertex of $\mathcal{O}$, that will separate the two fixed vertices on the boundary of $\mathcal{O}$. The second arm of the probe can touch an already known vertex. If this is not the case, then, since $\omega=\pi / 2$, we always have freedom to adjust the wedge in such a way, that the second arm will intersect the circle of one of not yet revealed vertices of the pentagon. The adversary then fixes the vertex in the center of the corresponding circle. Note, that this probe may reveal up to two new pieces of information about $\mathcal{O}$.

After every probe the adversary updates the curve $A$, and if new vertices were discovered, it also updates the $\omega$-cloud $\Omega$.

- If the algorithm spent 2 probes and revealed 4 vertices of the pentagon, then the fifth vertex is fixed by the adversary (as the center of the corresponding circle), but not revealed yet to the algorithm. The algorithm may guess its position though.

- If the algorithm spent 3 probes but there is still at least one not fixed vertex of the pentagon, then the adversary fixes all of them.

Either one of the two cases terminates the initialization step, and the adversary proceeds to the second stage of its protocol.

During the second stage of the strategy the adversary answers each query with already revealed vertices unless the direction of the probe contains two vertices $u$ and $v$ known to the algorithm, that are adjacent on $A$. In this situation one new vertex (between $u$ and $v$ on the boundary of $\mathcal{O}$ ) should be revealed or an edge $\overline{u v}$ must be confirmed. The adversary's strategy would not confirm any edge on this type of query until $n-1$ vertices have been revealed. Since we are not allowed narrow vertices, the adversary positions new vertices in such a way that their internal angle in the convex hull of already revealed vertices of $\mathcal{O}$ is bigger than $\omega$. Note, that for the particular case, when $\omega=\pi / 2$, fixed vertices that are unknown to the algorithm will be considered as revealed/known vertices.

When the algorithm knows $n-1$ vertices of $\mathcal{O}$ the adversary proceeds to the third stage of its strategy, during which the algorithm will be able to confirm edges. The adversary will not reveal the last, $n^{\text {th }}$ vertex, forcing the algorithm to inspect every consecutive pair of vertices on $A$ to check whether they are connected by an edge or there is a "missing" vertex between them. As a result, the adversary will reveal already known vertices and confirm edges.

By the final, fourth stage of the adversary's strategy, the algorithm knows $n-1$ vertices and $n-2$ edges of $\mathcal{O}$. There is left only one pair of adjacent vertices $u$ and $v$ for which the edge $\overline{u v}$ has not been verified. When the algorithm sets the direction of the probe to be the line $L_{\mathfrak{u} v}$, the adversary has no choice but to reveal the last vertex. The algorithm should spend at least two more probes to complete the polygon's boundary, because the number of vertices $n$ is unknown to the algorithm. The query session then is complete and the convex polygon is found.

The query for the adversary is a direction of the probe $\overrightarrow{\mathrm{L}}$. The adversary asks the algorithm about its next probe and outputs the result.

The input for the algorithm is the circle $\Psi$ and the point $p$. The algorithm also knows that the point $p$ is inside the unknown object $\mathcal{O}$. It then performs probes (i.e., gives the adversary the direction of the probe and gets results) and outputs a convex polygon $\mathcal{O}^{\prime}$. If $\mathcal{O}^{\prime}=\mathcal{O}$ then the output is correct. 


\section{Invariant (maintained by the adversary):}

1. A is a closed convex curve that is the intersection of the circle $\Psi$ and all $\omega$-probes made so far by the algorithm. $A$ is a circle or it consists of circular arcs and line segments.

2. $P$ is a set of points on $A$.

3. Each line segment of $A$ contains at least one and at most two points of $P$.

4. $F$ is a variable with value $F=\mid\{u \in P: u$ and $c c w(u)$ are not on the same line segment of $A\} \mid$, where $c c w(u)$ is the counter-clockwise successor of $u$ on $A$.

5. There exists a convex n-gon $\mathcal{O}$, such that each point of $P$ is a vertex of $\mathcal{O}$ and all internal angles of $\mathcal{O}$ are larger than $\omega$. If $\mathcal{O}$ is the object to be reconstructed, then the algorithm makes the same sequence of probes it has made so far.

6. After the initialization, $\mathrm{P}$ contains at least four points (for $0<\omega<\pi / 2$ ), and five or six points (for $\omega=\pi / 2$ ). All the internal angles of the polygon that have points of $\mathrm{P}$ as vertices, are larger than $\omega$.

\section{Adversary's strategy:}

1. Initialization: Choose $n \geqslant 5$, where $n$ denotes the number of vertices of the object to be reconstructed. On the first valid $\omega$-probe the adversary reveals two vertices $v_{1}$ and $v_{2}$, that are positioned as described above. Refer to Figure 12.3.

We consider two cases:

a) $0<\omega<\pi / 2$ : If the direction of the probe contains any of the four edges of the rectangle $\left\{v_{1}, t_{3}, v_{2}, t_{4}\right\}$, the points $t_{3}$ and $t_{4}$ move to avoid edge confirmation (as described above). If the probe reveals $t_{3}$ (or $t_{4}$ ), we fix both vertices and we rename $t_{3}$ into $v_{3}$ (and $t_{4}$ into $v_{4}$ ). We output only vertices found by the probe. Set $\mathrm{P}=$ $\left\{v_{1}, v_{2}, v_{3}, v_{4}\right\}, F=4$ and $A=\Psi \cap \omega$-wedge.

b) $\omega=\pi / 2$ : If the direction of the probe contains any of the five edges of the pentagon $\left\{v_{1}, c_{4}, c_{5}, v_{2}, c_{3}\right\}$, the points $c_{3}, c_{4}$ and $c_{5}$ move to avoid edge confirmation (as described above). If both points are fixed, reveal additional vertex $v$. Otherwise, answer queries with already known vertices or not fixed vertices of the pentagon until one of the following two happens:

i. If the algorithm spent 2 probes and revealed 4 vertices of the pentagon, then fix/rename the fifth vertex (as the center of the corresponding circle). Set $\mathrm{P}=$ $\left\{v_{1}, v_{2}, v_{3}, v_{4}, v_{5}\right\}$.

ii. If the algorithm spent 3 probes, then fix/rename all the vertices of the pentagon. Set $\mathrm{P}=\left\{v_{1}, v_{2}, v_{3}, v_{4}, v_{5}\right\}$. If an additional vertex $v$ was revealed then add $v$ to $\mathrm{P}$. Set $\mathrm{F}=|\mathrm{P}|$ and update $A$.

To answer all subsequent queries the adversary will use the following protocol:

2. While $|\mathrm{P}|<\mathrm{n}-1$ do the following:

Answer each query with already revealed vertices, unless the direction of the probe contains two known vertices $u, v \in P$, that are adjacent on $A$. In this situation the answer will be one new vertex $v^{\prime}$ (between $u$ and $v$ on the boundary of $\mathcal{O}$, such that $\left.\angle\left(v, v^{\prime}, u\right)>\omega\right)$ and one vertex $v^{\prime \prime}$ that is already known to the algorithm.

Add $v^{\prime}$ to $P$; set $F=F+1$. Update $A$.

3. While $F>1$ do the following: 
Answer each query with vertices from $P$, unless the direction of the probe contains $u, v \in P$, such that $v$ is a counter-clockwise successor of $u$ on $A$, and $u, v$ do not belong to the same straight-line edge of $A$. In the latter case confirm the edge $\overline{u v}$, set $F=F-1$ and update $A$.

4. Answer each query with vertices from $P$, unless the direction of the probe contains $u, v \in P$, such that $u$ and $v$ are adjacent on $A$ and they are not on the same straight-line edge of $A$ (in other words, the edge $\overline{u v}$ has not been verified). In this situation the answer would be one new vertex $v^{\prime}$ (between $u$ and $v$ on the boundary of $\mathcal{O}$ ) and one vertex $v^{\prime \prime}$ that is already known to the algorithm.

The algorithm should spend at least two more probes to complete the boundary of the polygon, because the number of vertices $n$ is unknown to the algorithm. The query session is then complete and the polygon is found.

It follows from this protocol that the invariant is correctly maintained. With the above strategy any algorithm will spend at least $2 n-2$ probes for any $\omega$ in the range $0<\omega<\pi / 2$, and at least $2 n-3$ probes for $\omega=\pi / 2$.

THEOREM 12.5. Given an $\omega$-wedge, where $0<\omega<\pi / 2$, for every algorithm, there exists a convex polygon $\mathcal{O}$ (whose angles are strictly bigger than $\omega$ ), such that $2 \mathrm{n}-2 \mathrm{\omega}$-probes are required to determine its shape. For the case when $\omega=\pi / 2,2 \mathrm{n}-3 \omega$-probes are required to determine $\mathcal{O}$.

Proof. To prove this, consider the quantity

$$
\Phi=2|\mathrm{P}|-\mathrm{F} .
$$

At the start of the strategy $P$ is empty and $F=0$, so $\Phi=0$.

Let us first analyze the case when $0<\omega<\pi / 2$. At the initial step any algorithm will spend at least 2 probes to reveal 4 vertices. After initialization $|P|=4$ and $F=4$, thus $\Phi=4$.

Every iteration of the while-loop in the second step of the protocol either does not change the values of $|\mathrm{P}|$ and $\mathrm{F}$, or increases $|\mathrm{P}|$ by 1 and increases $\mathrm{F}$ by 1 . Therefore, $\Phi$ increases by at most one on every probe made by the algorithm. This while-loop makes at least $n-5$ iterations (forcing the algorithm to spend at least $n-5$ probes) resulting in $\Phi=n-1$.

Every iteration of the while-loop in the third step of the protocol does not change the value of $|\mathrm{P}|$, but decreases the value of $\mathrm{F}$ by at most 1 . Therefore, $\Phi$ increases by at most one on every probe made by the algorithm. This while-loop makes at least $n-2$ iterations (forcing the algorithm to spend at least $n-2$ probes) resulting in $\Phi=2 n-3$.

In the final step of the protocol, any algorithm will spend at least 3 probes to complete the boundary of the polygon. We prove below (see Claims 1 and 2) that, after termination we have $|P|=n, F=0$ and $\Phi=2 \mathrm{n}$.

To conclude, the number of probes made by any algorithm is at least $2+(n-5)+(n-2)+3=$ $2 n-2$.

For the case when $\omega=\pi / 2$, the initialization step can have two outcomes:

1. At most two probes were spent, that resulted in $|P|=5, F=5$ and thus $\Phi=5$.

2. At most three probes were spent, that resulted in $|P|=6, F=6$ and thus $\Phi=6$.

The first while-loop makes at least $n-6$ iterations for the first outcome (forcing the algorithm to spend at least $n-6$ probes), and at least $n-7$ iterations for the second outcome (forcing the algorithm to spend at least $n-7$ probes) resulting in $\Phi=n-1$. The analysis for the third and fourth step of the protocol are identical to the one given above. So, the number of probes made by any algorithm is at least $2+(n-6)+(n-2)+3=2 n-3$ (for the first outcome of the initialization), and $3+(n-7)+$ $(n-2)+3=2 n-3$ (for the second outcome). 


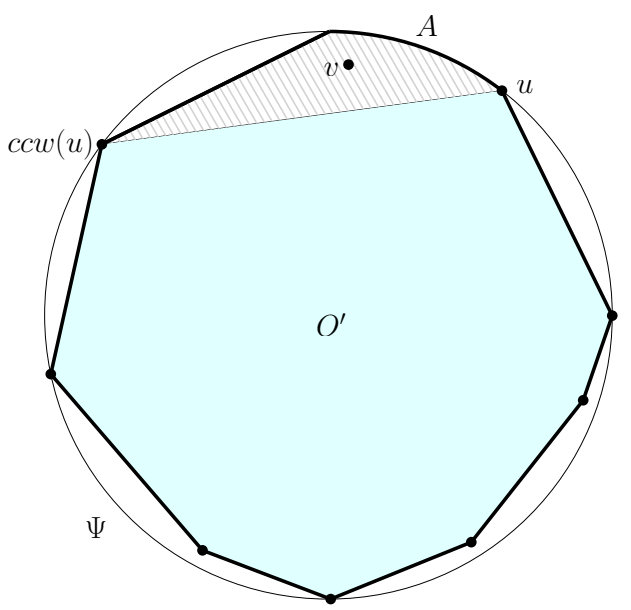

Figure 12.4: The figure shows an area (tiled with grey lines) not explored by the algorithm. The curve $A$ is shown in bold; the object $\mathcal{O}^{\prime}$, returned by the algorithm, is depicted in cyan.

Claim 1: When the algorithm terminates: $F=0$.

Proof. Assume to the contrary that the algorithm returned an object $\mathcal{O}^{\prime}$ (where $\mathcal{O}^{\prime}$ is a convex hull of $P)$, but $F \geqslant 1$. When $F$ is not empty, there exists a vertex $u \in P$ such that $u$ and $\operatorname{ccw}(u)$ are not on the same line segment of $A$. Since $A$ is convex, the line segment $\overline{u, c c w(u)}$ is completely inside $A$ and its intersection with $A$ is the set of points $\{u, c c w(u)\}$. Refer to Figure 12.4. There exists a vertex $v$ in the interior of the region, consisting of all points in $A$, that are to the right of $\overline{u, c c w}(u)$. The adversary can claim that the object $\mathcal{O}$ is the convex hull of $\mathrm{P} \bigcup\{v\}$. If the adversary gives the convex hull of $\mathrm{P} \bigcup\{v\}$ to the algorithm as an object, the algorithm will make exactly the same set of probes as it did for the convex hull of $\mathrm{P}$, and therefore will give the same output $\mathrm{O}^{\prime}$ for different objects $\mathcal{O}$ and $\mathcal{O}^{\prime}$. Since $\mathcal{O} \neq \mathcal{O}^{\prime}$ the algorithm is not correct.

Claim 2: When the algorithm terminates: $|\mathrm{P}|=\mathrm{n}$.

Proof. Assume that the algorithm returned an object $\mathcal{O}^{\prime}$ such that $|\mathrm{P}|<\mathrm{n}$ (notice, that $\mathrm{P}$ cannot be bigger than $n$ ). According to the invariant, there exists a convex $n$-gon $\mathcal{O}$ such that each point of $P$ is a vertex of $\mathcal{O}$ and the algorithm would make the same sequence of probes on $\mathcal{O}$ as it has made on $\mathcal{O}^{\prime}$. It is clear that $\mathcal{O} \neq \mathcal{O}^{\prime}$ and thus the algorithm is not correct. 


\section{POLYGONS WITH NARROW VERTICES}

\section{3}

This chapter addresses the reconstruction of convex polygons that have narrow vertices. We showed in Chapter 11 that the number of narrow vertices cannot exceed 3 (refer to Observation 11.1) and if $\omega<\pi / 3$ then the number of narrow vertices can be at most 2 (refer to Observation 11.2).

Recall that the result of a valid probe is the coordinates of the apex of the probe. Notice that if the ray in the direction of the probe enters $\mathcal{O}$ via narrow vertex, then the apex of this probe belongs to the $\omega$-cloud $\Omega$ of the polygon. If the polygon does not have any narrow vertices then the $\omega$-cloud and the boundary of the polygon are disjoint.

Let us first investigate the problem of reconstructing polygons that have exactly one narrow vertex.

\subsection{POLYGONS WITH EXACTLY ONE NARROW VERTEX}

Let $v_{B}$ be the only narrow vertex of $\mathcal{O}$. Let $a$ and $b$ be two points and let $L_{a b}$ be the line through $a$ and $b$. Let $\overrightarrow{L_{a b}}$ be the direction of a probe $W$, directed from $a$ to $b$. Notice, that if we shoot a probe along $\overrightarrow{L_{v_{B}}}$, then the $\omega$-probe will stop with the apex $q$ touching the vertex $v_{B}$. The arms of the probe may or may not touch the boundary of the polygon (see Figures $10.2 \mathrm{C}$ and 13.1a).

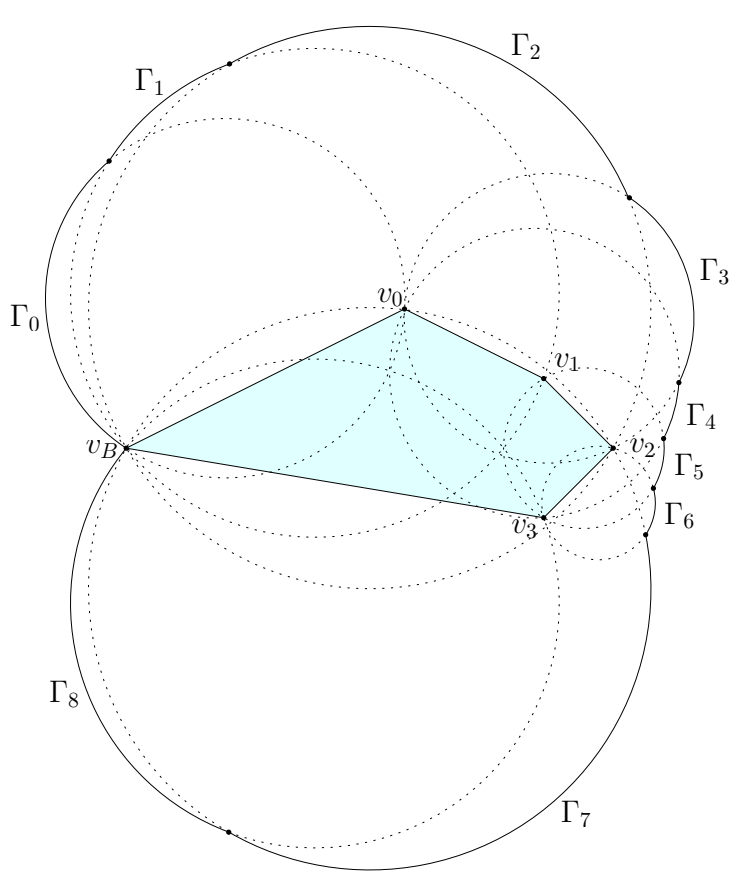

(a) $v_{\mathrm{B}}$ is the only narrow vertex of $\mathcal{O}$.

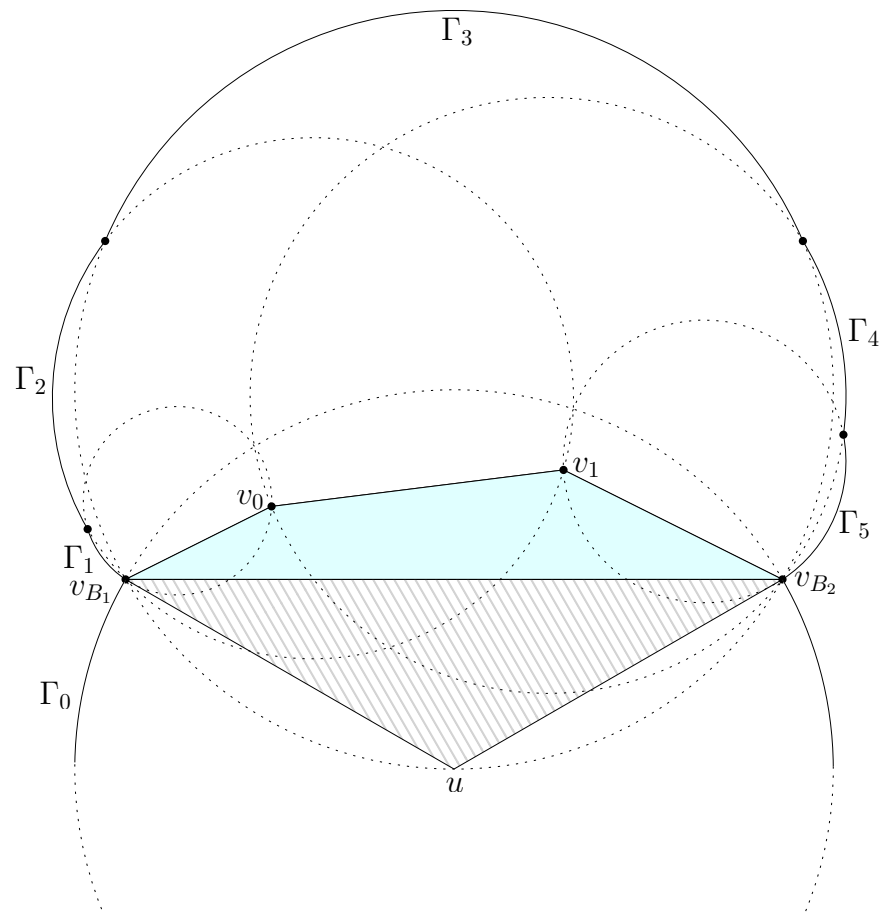

(b) $\mathcal{O}$ has two narrow vertices. The triangle $\triangle\left(v_{\mathrm{B}_{1}}, v_{\mathrm{B}_{2}}, \mathrm{u}\right)$ may or may not contain vertices of $\mathcal{O}$, other that $v_{\mathrm{B}_{1}}$ and $v_{\mathrm{B}_{2}}$.

Figure 13.1: Case studies of narrow vertices of $\mathcal{O}$. In both examples, $\omega=60^{\circ}$. 
Look at the example of Figure 13.1a. Assume that we have found the vertices $v_{\mathrm{B}}$ and $v_{0}$, but the vertices $v_{1}, v_{2}$ and $v_{3}$ are still unknown. Suppose that our next step is to check whether $\overline{v_{\mathrm{B}} v_{0}}$ is an edge. Since we have no control over the rotation of the arms, the probe in the direction $\overrightarrow{\mathrm{L}_{v_{B} v_{0}}}$ might give us no new information other than to confirm that $v_{\mathrm{B}}$ is a narrow vertex (in case we did not know already). The solution is to probe in the opposite direction, namely $\overrightarrow{\mathrm{L}_{v_{0} v_{B}}}$. This will confirm the edge $\overline{v_{\mathrm{B}} v_{0}}$ (and reveal $\left.v_{2}\right)$.

Consider again the example of Figure 13.1a, but now assume that we have found $v_{\mathrm{B}}$ and $v_{1}$, but the vertices $v_{0}, v_{2}$ and $v_{3}$ are still unknown. Our goal is to deny the existence of the edge $\overline{v_{\mathrm{B}} v_{1}}$ by revealing the vertex $v_{0}$. But we cannot rely on the probing in the direction $\overrightarrow{\mathrm{L}_{v_{B} v_{1}}}$. In the worst case it will not return $v_{0}$. As in the previous example, the probe in the opposite direction will work. The probe along $\overrightarrow{\mathrm{L}_{v_{1}} v_{\mathrm{B}}}$ returns two new vertices, namely $v_{0}$ and $v_{2}$.

We may conclude, that having one narrow vertex in the polygon is not a problem. Once a narrow vertex is identified, all other elements of $\mathcal{O}$ can be found by using algorithm from Section 12.1 while probing in the directions other then those whose first point of intersection with $\mathcal{O}$ is the narrow vertex. Note that we may discover a narrow vertex by probing in different directions. But only the probe along $\overrightarrow{\mathrm{L}_{v_{B}} \mathrm{a}}$ (where $\mathrm{a}$ is any point interior to $\mathcal{O}$ ) can identify $v_{\mathrm{B}}$ as a narrow vertex. Hence, we may spend one additional probe per narrow vertex to reconstruct the polygon $\mathcal{O}$. The algorithm that reconstructs convex polygon with exactly one narrow vertex is given below in the Section 13.3. The algorithm reconstructs $\mathcal{O}$ by using not more than $2 n-1$ probes. This upper bound is proved in Theorem 13.1.

\subsection{POLYGONS WITH TWO OR THREE NARROW VERTICES}

The problem arises when a polygon has more than one narrow vertex. Refer to Figure 13.1b. Assume we have found two narrow vertices $v_{\mathrm{B}_{1}}$ and $v_{\mathrm{B}_{2}}$, but all other elements of $\mathcal{O}$ are still unknown. Our previous strategy to shoot a probe along $\overrightarrow{\mathrm{L}_{\mathrm{B}_{1} v_{\mathrm{B}_{2}}}}$ or in the opposite direction (along $\overrightarrow{\mathrm{L}_{\mathrm{B}_{2} v_{\mathrm{B}_{1}}}}$ ) does not work.

If there is a vertex $v$ of $\mathcal{O}$ that is between $v_{\mathrm{B}_{1}}$ and $v_{\mathrm{B}_{2}}$ in a clockwise order around the boundary of $\mathcal{O}$, then there are at least two arcs $\Gamma_{v}$ and $\Gamma_{v}^{\prime}$ (supported by $v$ ) that belong to the part of the $\Omega$ cloud enclosed between two pivots $\nu_{\mathrm{B}_{1}}$ and $\nu_{\mathrm{B}_{2}}$ in a clockwise order around the cloud. (Recall from Definition 10.5 that a pivot point is an intersection point of two consecutive circular arks of the $\Omega$ cloud). If we probe in the direction toward the $\operatorname{arc} \Gamma_{v}$ then we will find $v$. We know that $\Gamma_{v}$ is on the cloud between $v_{\mathrm{B}_{1}}$ and $\Gamma_{v}^{\prime}$ (clockwise), but we do not know how big it is. In other words, we do not know how close to $v_{\mathrm{B}_{1}}$ we should aim.

Let $\varepsilon>0$ be a fixed real number. We suppose, that for every pair of narrow vertices $\nu_{\mathrm{B}_{1}}$ and $\nu_{\mathrm{B}_{2}}$ of

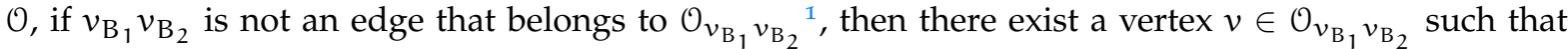
$\angle\left(v_{\mathrm{B}_{2}}, v, v_{\mathrm{B}_{1}}\right) \leqslant \pi-\varepsilon$. According to this hypothesis, one of the angles $\angle\left(v, v_{\mathrm{B}_{1}}, v_{\mathrm{B}_{2}}\right)$ or $\angle\left(v_{\mathrm{B}_{1}}, v_{\mathrm{B}_{2}}, v\right)$ is at least $\varepsilon / 2$. Assume, without loss of generality, that $\angle\left(v_{\mathrm{B}_{1}}, v_{\mathrm{B}_{2}}, v\right) \geqslant \varepsilon / 2$. Thus, if we shoot a probe

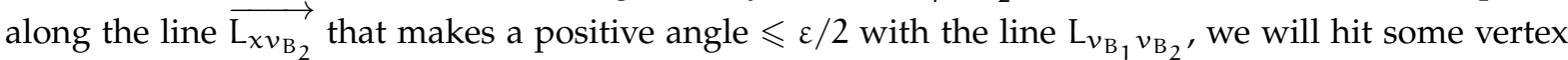

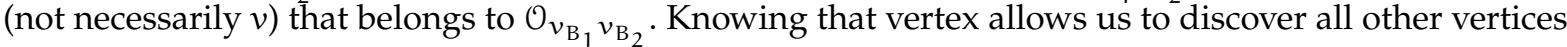
on the boundary $\mathcal{O}_{v_{B_{1}}} v_{\mathrm{B}_{2}}$ by using the same strategy as before when we had only one narrow vertex.

Since we do not know which one of the two angles $\angle\left(v_{\mathrm{B}_{1}}, v_{\mathrm{B}_{2}}, v\right)$ or $\angle\left(v, v_{\mathrm{B}_{1}}, v_{\mathrm{B}_{2}}\right)$ is bigger than $\varepsilon / 2$, an additional probe (along the line $\overrightarrow{\mathrm{L}_{x v_{B_{1}}}}$ that makes a negative angle $\leqslant \varepsilon / 2$ with the line $\mathrm{L}_{v_{B_{2}}} v_{\mathrm{B}_{1}}$ ) may be required to identify $v$. If both probes return no new information, then we confirm an edge from $v_{\mathrm{B}_{2}}$ to $v_{\mathrm{B}_{1}}$ in the counter clockwise order around the boundary of $\mathcal{O}$.

1 For the rest of this part of the thesis, $\mathcal{O}_{v_{1} v_{2}}$ denotes the part of the boundary of $\mathcal{O}$ in a counter-clockwise order from $v_{1}$ to $v_{2}$ including $v_{1}$ and $v_{2}$. 
But what if $\varepsilon$ is unknown? We may try to guess $\varepsilon$. We may choose an initial (very small) value for

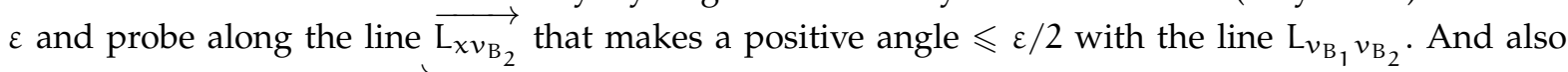
probe along the line $\overrightarrow{\mathrm{L}_{\chi^{\prime} v_{B_{1}}}}$ that makes a negative angle $\leqslant \varepsilon / 2$ with the line $L_{v_{B_{2}}} v_{B_{1}}$. If no vertex is discovered, we reduce the value of $\varepsilon$ by a factor of two and we repeat the above procedure until we hit some vertex that belongs to $\mathcal{O}_{v_{\mathrm{B}_{1}}} v_{\mathrm{B}_{2}}$. This strategy, unfortunately, can take an infinite number of

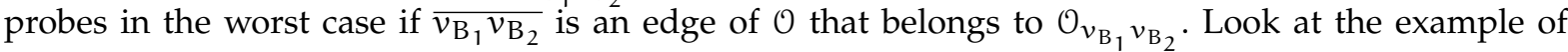
Figure $13 . \mathrm{Ib}$ and assume that the only vertices of $\mathcal{O}$ inside the triangle $\triangle\left(v_{\mathrm{B}_{1}}, v_{\mathrm{B}_{2}}, \mathrm{u}\right)$ are $v_{\mathrm{B}_{1}}$ and $v_{\mathrm{B}_{2}}$ and we probe along a line $\overrightarrow{\mathrm{L}_{x v_{\mathrm{B}_{2}}}}$ that approaches the edge $\overline{{\overline{\mathrm{B}_{1}}}_{1} v_{\mathrm{B}_{2}}}$ from below.

In other words, if $\varepsilon$ is unknown, the edge $\overline{v_{\mathrm{B}_{1}} v_{\mathrm{B}_{2}}}$ cannot be verified and thus $\mathcal{O}$ cannot be reconstructed precisely.

Now we are ready to present an algorithm for the reconstruction of a convex polygon whose internal angles may be smaller than $\omega$.

\section{$13 \cdot 3$ ALGORITHM}

In this section we show an algorithm that reconstructs general convex polygons. For polygons with two or three narrow vertices the algorithm requires a real number $\varepsilon>0$ as a part of an input. Otherwise the polygon may not be reconstructed precisely. Upper bound on the number of probes spent by the algorithm is given in Theorem 13.1.

\section{Invariant:}

1. $\mathrm{Q}$ is a convex polygon.

2. Each vertex of $Q$ is a vertex of $\mathcal{O}$ (and, therefore, $Q$ is contained in $\mathcal{O}$ ).

3. Each vertex $u$ of $Q$ stores a Boolean variable $f \operatorname{lag}(u)$; if $\operatorname{flag}(u)=$ true, then $u$ and its counterclockwise successor in $\mathrm{Q}$ form an edge of $\mathcal{O}$.

4. Each vertex $u$ of $Q$ stores a Boolean variable $B$ vertex $(u)$; if $B$ vertex $(u)=$ true, then $u$ is a narrow vertex in $\mathcal{O}$.

5. $\mathrm{F}$ is a variable with value $\mathrm{F}=|\{\mathrm{u} \in \mathrm{Q}: \operatorname{flag}(\mathrm{u})=f a l s e\}|$.

\section{Algorithm:}

To initialize the algorithm, choose an arbitrary line $\vec{L}$ that contains $p$. Let $\left(q, H_{1}, H_{2}, p_{1}, p_{2}\right)$ be the outcome of the probe along $\overrightarrow{\mathrm{L}}$.

- If $p_{1} \neq p_{2}$, initialize $Q$ as the polygon consisting of the vertices $p_{1}$ and $p_{2}$, set $f l a g\left(p_{1}\right)=$ false, flag $\left(\mathrm{p}_{2}\right)=$ false, $\mathrm{Bvertex}\left(\mathrm{p}_{1}\right)=$ false, $\mathrm{Bvertex}\left(\mathrm{p}_{2}\right)=$ false and $\mathrm{F}=2$.

- Otherwise, if $p_{1}=p_{2}=q$, initialize $Q$ as the polygon consisting of one vertex $q$, set flag $(q)=$ false, Bvertex $(q)=$ true, and $\mathrm{F}=1$. Let $\left(\mathrm{q}^{\prime}, \mathrm{H}_{1}^{\prime}, \mathrm{H}_{2}^{\prime}, \mathrm{p}_{1}^{\prime}, \mathrm{p}_{2}^{\prime}\right)$ be the outcome of the probe along the bisector of the first probe in the direction from inside the first wedge toward q.

- If $p_{1}^{\prime} \neq p_{2}^{\prime}$ then:

* if $p_{1}^{\prime} \neq q$, replace $Q$ by the convex hull of $Q \cup\left\{p_{1}^{\prime}\right\}$ and set flag $\left(p_{1}^{\prime}\right)=$ false, Bvertex $\left(\mathrm{p}_{1}^{\prime}\right)=$ false and $\mathrm{F}=\mathrm{F}+1$.

* if $\mathrm{p}_{2}^{\prime} \neq \mathrm{q}$, replace $\mathrm{Q}$ by the convex hull of $\mathrm{Q} \cup\left\{\mathrm{p}_{2}^{\prime}\right\}$ and set flag $\left(\mathrm{p}_{2}^{\prime}\right)=$ false, Bvertex $\left(\mathrm{p}_{2}^{\prime}\right)=$ false and $\mathrm{F}=\mathrm{F}+1$. 
- Otherwise, if $p_{1}^{\prime}=p_{2}^{\prime}=q^{\prime}$, do the following: if $q=q^{\prime}$ set $F=0$. Otherwise, replace $Q$ by the convex hull of $\mathrm{Q} \cup\left\{\mathbf{q}^{\prime}\right\}$ and set flag $\left(\mathbf{q}^{\prime}\right)=$ false, $\operatorname{Bvertex}\left(\mathbf{q}^{\prime}\right)=$ true and $\mathrm{F}=\mathrm{F}+1$.

While $F \neq 0$, do the following:

1. a) If there is a vertex $u$ in $Q$ such that $\operatorname{flag}(u)=\operatorname{Bvertex}(u)=$ false, let $u$ be such a vertex (give priority to vertices whose internal angles in $Q$ are bigger than $\omega$ ), and let $v$ be the counter-clockwise successor of $u$ in $Q$.

b) Else, if there is a vertex $u$ in $Q$ for which $B$ vertex $(u)=$ false and whose clockwise successor $v$ in Q satisfy the following properties: $\operatorname{flag}(v)=$ false and Bvertex $(v)=\operatorname{true}$, then let $u$ be such a vertex, and let $v$ be its clockwise successor in $Q$.

c) Else, do the following:

If we are not given an $\varepsilon$, stop the algorithm and return Q. Otherwise, take a pair of arbitrary adjacent vertices $u$ and $v$ in $Q$ for which flag $(u)=$ false, Bvertex $(u)=$ $\operatorname{Bvertex}(v)=$ true and $v$ is the counter-clockwise successor of $u$ in $Q$. Let $\overrightarrow{\mathrm{Luv}_{u}}$ be the line through $u$ and $v$ that is directed from $u$ to $v$. Let $\overrightarrow{\mathrm{L}}$ be the line $\overrightarrow{\mathrm{L}_{u v}}$ that is rotated to the left by $\varepsilon / 2$ degrees around vertex $v$. Let $\left(q, H_{1}, H_{2}, p_{1}, p_{2}\right)$ be the outcome of the probe along $\overrightarrow{\mathrm{L}}$.

i. If $p_{1} \neq v$, then insert $p_{1}$ into $Q$ between $u$ and $v$, and set $\operatorname{flag}\left(p_{1}\right)=$ false, Bvertex $\left(\mathrm{p}_{1}\right)=$ false and $\mathrm{F}=\mathrm{F}+1$.

ii. If $p_{1}=v$, then let $\overrightarrow{\mathrm{L}^{\prime}}$ be the line $\overrightarrow{\mathrm{L}_{v u}}$ that is rotated to the right $\varepsilon / 2$ degrees around vertex $u$. Let $\left(q^{\prime}, H_{1}^{\prime}, H_{2}^{\prime}, p_{1}^{\prime}, p_{2}^{\prime}\right)$ be the outcome of the probe along $\overrightarrow{L^{\prime}}$.

- If $p_{2}^{\prime} \neq u$ then insert $p_{2}^{\prime}$ into $Q$ between $u$ and $v$, and set flag $\left(p_{2}^{\prime}\right)=$ false, Bvertex $\left(\mathrm{p}_{2}^{\prime}\right)=$ false and $\mathrm{F}=\mathrm{F}+1$.

- If $\mathrm{p}_{2}^{\prime}=\mathrm{u}$ then set $\operatorname{flag}(\mathrm{u})=$ true and $\mathrm{F}=\mathrm{F}-1$.

Skip steps 2, 3 and 4 and continue to the next iteration of the while-loop.

2. Let $\vec{L}$ be the line through $u$ and $v$ that is directed from $u$ to $v$. Let $\left(q, H_{1}, H_{2}, p_{1}, p_{2}\right)$ be the outcome of the probe along $\overrightarrow{\mathrm{L}}$.

If $\operatorname{flag}(\mathrm{u})=$ true, rename $\mathrm{p}_{1}$ into $\mathrm{p}_{2}$ and $\mathrm{p}_{2}$ into $\mathrm{p}_{1}$.

If $p_{1}=p_{2}=q=u$, then set Bvertex $(u)=$ true and go to step 1 .

3. a) If $p_{1}=u$, then

- if $\operatorname{flag}(\mathrm{u})=$ true, then set $f \operatorname{lag}(v)=$ true and $\mathrm{F}=\mathrm{F}-1$.

- otherwise, set $\operatorname{flag}(\mathrm{u})=$ true and $\mathrm{F}=\mathrm{F}-1$.

b) If $p_{1} \neq u$, insert $p_{1}$ into $Q$ between $u$ and $v$, and set flag $\left(p_{1}\right)=$ false, Bvertex $\left(p_{1}\right)=$ false and $\mathrm{F}=\mathrm{F}+1$.

4. a) If $p_{2}=u$, set $\operatorname{flag}(v)=$ true and $F=F-1$.

b) If $p_{2} \neq u$ and $p_{2}$ is not a vertex of $Q$, then replace $Q$ by the convex hull of $Q \cup\left\{p_{2}\right\}$, and set flag $\left(\mathrm{p}_{2}\right)=$ false, Bvertex $\left(\mathrm{p}_{2}\right)=$ false and $\mathrm{F}=\mathrm{F}+1$.

It follows from the algorithm that the invariant is correctly maintained. We show that the algorithm terminates, by proving the following theorem.

THEOREM 13.1 (Upper Bound). Given $\omega$-wedge, with $0<\omega \leqslant \pi / 2$, a convex polygon $\mathcal{O}$, and $\varepsilon>0$ (such

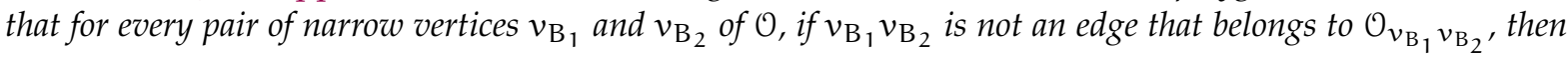


there exists a vertex $v \in \mathcal{O}_{v_{\mathrm{B}_{1}} v_{\mathrm{B}_{2}}}$ such that $\left.\angle\left(v_{\mathrm{B}_{2}}, v, v_{\mathrm{B}_{1}}\right) \leqslant \pi-\varepsilon\right)$. Let $\mathrm{N}_{\mathrm{B}}$ be the number of narrow vertices of $\mathcal{O}$ and let:

$$
P_{B}= \begin{cases}-1, & N_{B}=0, \\ -1, & N_{B}=1, \\ 2, & N_{B}=2, \\ 3, & N_{B}=3 .\end{cases}
$$

The above algorithm reconstructs $\mathcal{O}$ using at most $2 \mathrm{n}-1+\mathrm{N}_{\mathrm{B}}+\mathrm{P}_{\mathrm{B}}$ w-probes.

Proof. Let $\mathrm{n}$ denote the number of vertices of $\mathcal{O}$. Consider the quantity

$$
\Phi=2|\mathrm{Q}|+\mathrm{N}_{\mathrm{B}}^{\prime}-\mathrm{F},
$$

where $|\mathrm{Q}|$ denotes the number of vertices of $\mathrm{Q}, \mathrm{N}_{\mathrm{B}}^{\prime}$ denotes the number of narrow vertices of $\mathrm{Q}$, $0 \leqslant N_{B}^{\prime} \leqslant 3$ (refer to Observation 11.1). The quantity $\Phi$ represents the number of units of information we need to obtain in order to successfully reconstruct $\mathcal{O}$. These units of information are vertices, edges and narrow vertices of $\mathcal{O}$ that were identified as such by the algorithm. At the initial step of the algorithm, after the first probe, we have $\Phi=2$ : either the probe returned two vertices (in which case $\left.|\mathrm{Q}|=2, \mathrm{~N}_{\mathrm{B}}^{\prime}=0, \mathrm{~F}=2\right)$, or it returned one narrow vertex $\left(|\mathrm{Q}|=1, \mathrm{~N}_{\mathrm{B}}^{\prime}=1, \mathrm{~F}=1\right)$. In the first case, the algorithm proceeds to the while-loop step, while in the second case, one more probe is required. This additional probe reveals either two new vertices, or one new vertex, or one new narrow vertex.

During every iteration of the while-loop, the algorithm reveals at least one vertex or one edge of $\mathcal{O}$ or marks an already known vertex as a narrow vertex. Therefore, in every iteration, the value of $\Phi$ increases by at least one. Notice also that if $\epsilon$ is known, then the step (1.c) of the algorithm increases the value of $\Phi$ by exactly one, but may use up to two probes. Fortunately, this step of the algorithm cannot be executed more than three times (refer to Observation 11.1).

Since at any moment we have $\Phi \leqslant 2 n+N_{B}$ (where $N_{B}$ stands for the number of narrow vertices of $0,0 \leqslant N_{B} \leqslant 3$ (refer to Observation 11.1)), it follows that the while-loop makes at most $2 n-2+N_{B}$ iterations and thus, the algorithm terminates.

After the very first probe $\Phi=2$. If this probe revealed two vertices, then the first iteration of the while-loop increases the value of $\Phi$ either by two (in which case two new vertices or one new vertex and one new edge were revealed), or by one (in which case an already known vertex was marked as a narrow vertex, making the number of non-revealed narrow vertices of $\mathcal{O}$ to decrease by one). Thus, the algorithm terminates after at most $2 n-3+N_{B}$ additional iterations, making a total of at most $2 n-1+N_{B}+P_{B}$ probes. Notice, that if $\mathcal{O}$ has no narrow vertices, then the first iteration of the whileloop always increases $\Phi$ by two, and thus the while-loop makes at most $2 n-4$ additional iterations, that results in at most $2 n-2$ probes. This is why $P_{B}$ is set to -1 for polygons without narrow vertices. Similarly, if the polygon has exactly one narrow vertex, that was discovered by the first probe and marked as a narrow vertex during the first iteration of the while-loop, then the second iteration of the loop reveals two new pieces of information. Those two new pieces can be either two new vertices or one new vertex and one new edge. Thus, the while-loop makes at most $2 n-4$ additional iterations. Hence, the algorithm in this case makes at most $2 n-1$ probes to reconstruct the polygon with one narrow vertex.

If the first probe of the algorithm revealed a narrow vertex, then an additional probe is required during the initialization step. This second probe may reveal:

- two new vertices, making $\Phi=4\left(|\mathrm{Q}|=3, \mathrm{~N}_{\mathrm{B}}^{\prime}=1\right.$ and $\left.\mathrm{F}=3\right)$.

- one new vertex, making $\Phi=3\left(|\mathrm{Q}|=2, \mathrm{~N}_{\mathrm{B}}^{\prime}=1\right.$ and $\left.\mathrm{F}=2\right)$. 
- one new narrow vertex, making $\Phi=4\left(|\mathrm{Q}|=2, \mathrm{~N}_{\mathrm{B}}^{\prime}=2\right.$ and $\left.\mathrm{F}=2\right)$.

Notice, that in either case, the number of non-revealed narrow vertices of $\mathcal{O}$ decreases by $N_{B}^{\prime}$.

It then requires at most $2 n-3+N_{B}$ iterations of the while-loop to reconstruct $\mathcal{O}$, which results in a total of at most $2 n-1+N_{B}+P_{B}$ probes. Notice that if $\mathcal{O}$ has exactly one narrow vertex that was discovered and marked by the first probe, then one of the following probes will discover two new pieces of information. This probe can be either the second probe of the initialization step or the first iteration of the loop. Two new pieces of information can be either two new vertices or one new vertex and one new edge. Thus, the algorithm makes at most $2 n-4$ additional probes, and reconstructs the polygon with one narrow vertex by using at most $2 n-1$ probes.

After termination, we have $F=0$. It then follows from the invariant that, at that moment, $Q$ is equal to $\mathcal{O}$. Finally, the number of probes made by the algorithm is at most $2 n-1+N_{B}+P_{B}$.

\subsection{LOWER BOUND FOR GENERAL POLYGONS}

We show a lower bound on the number of probes needed to reconstruct a convex n-gon by presenting an adversarial argument. In our proof, the adversary sets the number of narrow vertices in $\mathcal{O}$ before the first probe of the algorithm and cannot change its decision during the run of the algorithm. Thus, the adversary have to induce the predetermined number of narrow vertices in $\mathcal{O}$ despite the probing strategy of the algorithm. The algorithm, in its turn, may correctly guess the number of narrow vertices and use its best strategy for that specific number of narrow vertices. Thus it may reconstruct $\mathcal{O}$ by using less probes, than required for any other algorithm that does not know about constraints on $\mathcal{O}$.

We prove that, if the algorithm knows in advance the exact number of narrow vertices $0 \leqslant N_{B} \leqslant 3$ in $\mathcal{O}$, the lower bound on the number of probes required to reconstruct $\mathcal{O}$ is

$$
\begin{aligned}
& 2 n-2 \text { for } N_{B}=0, \\
& 2 n-1 \text { for } N_{B}=1, \\
& 2 n+2 \text { for } N_{B}=2 \text { and } N_{B}=3 .
\end{aligned}
$$

The lower bound for zero or one narrow vertex is tight with the upper bound (refer to Theorems 12.1, 12.4, 12.5 and 13.1). The lower bound for $N_{B}=2$ (respectively $N_{B}=3$ ) is smaller by one probe (respectively, by 3 probes) than the upper bound given in Theorem 13.1. We will show later in this section how to improve our algorithm for the case when the number of narrow vertices of $\mathcal{O}$ is provided to the algorithm. In this case, our lower bounds are tight. Alternatively, if the adversary is allowed to change its decision about the number of narrow vertices in $\mathcal{O}$ during the run of the algorithm, it can make the lower bound equal to the upper bound for any number of narrow vertices.

For now, assume that the adversary sets the number of narrow vertices in $\mathcal{O}$ before the first probe of the algorithm and does not change its decision during the execution of the algorithm. The adversary's strategy that forces at least $2 n-2 \omega$-probes from any algorithm which reconstructs $\mathcal{O}$ with no narrow vertices is given in Section 12.3. Here we describe strategies for polygons with one, two or three narrow vertices. We assume, as before, that the algorithm is deterministic and does not repeat the same probe during same reconstruction session.

\subsubsection{Lower Bound for Polygons with One Narrow Vertex}

We begin with the case where $N_{B}=1$. We will prove a lower bound of $2 n-1 \omega$-probes. The adversary defines a circle $\Psi$, a point $p$ as a center of $\Psi$, chooses $n \geqslant 3$ and sets $N_{B}=1$. The adversary maintains a closed convex curve $A$, that is the boundary of an intersection of all $\omega$-probes made by the algorithm and the circle $\Psi$. Initially, $A$ is the circle $\Psi$. The vertices of $\mathcal{O}$ revealed during the execution of the algorithm remain fixed, defining sections of the curve that cannot be changed. At the end of a query session $A$ is a convex polygon $\mathcal{O}$. 
The query made by the algorithm consists of a direction $\overrightarrow{\mathrm{L}}$ for the probe. Assume that the probe is valid and $\overrightarrow{\mathrm{L}}$ contains $p$. (We treat non-valid probes similarly to the strategy described in Section 12.3). The adversary stops the probe at any moment after the apex $q$ of the probe enters the interior of $\Psi$, but before it reaches $p$. Let $\left(q, H_{1}, H_{2}, p_{1}, p_{2}\right)$ be the outcome of the probe along $\vec{L}$ returned by the adversary, such that $\mathrm{H}_{1}$ (respectively $\mathrm{H}_{2}$ ) makes a negative (respectively positive) angle of $\omega / 2$ with $\overrightarrow{\mathrm{L}} ; \mathrm{q}=\mathrm{p}_{1}=\mathrm{p}_{2}$. In other words, on the first valid probe of the algorithm, the adversary reveals the narrow vertex $v_{\mathrm{B}}=\mathrm{q}$ of $\mathcal{O}$. Refer to Figure 13.2.

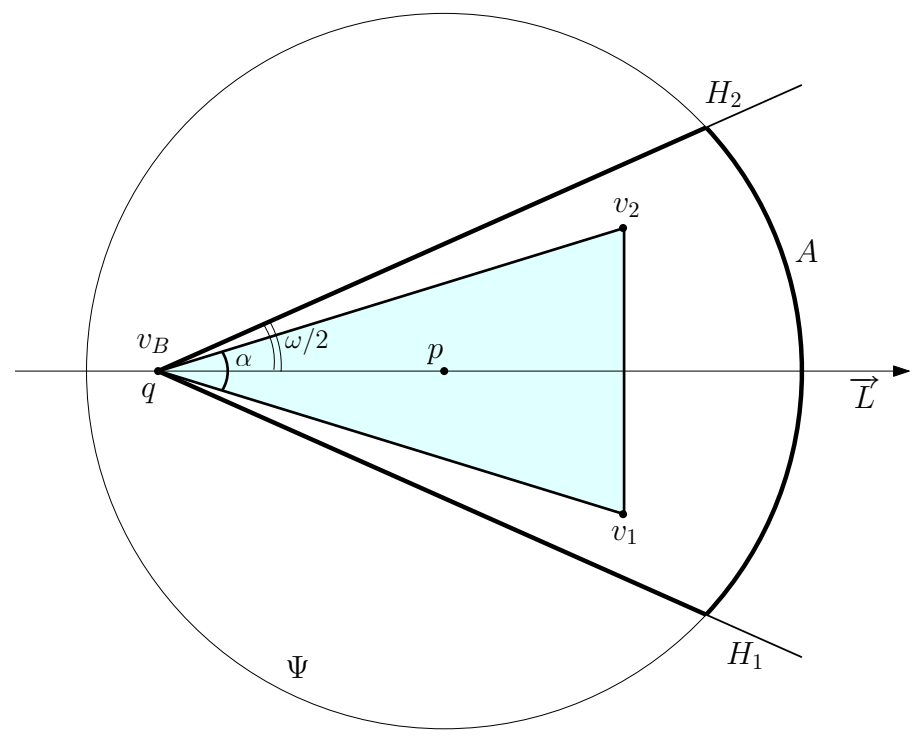

Figure 13.2: Initialization step for $0<\omega<\pi / 2$. Adversary induces exactly one narrow vertex in $\mathcal{O}$ by revealing it at the first valid probe of the algorithm. The closed curve $A$ is highlighted in bold. $\mathcal{O}$ is fully contained within $A$.

To ensure that $v_{\mathrm{B}}$ is the only narrow vertex of $\mathcal{O}$, the adversary creates a frame polygon (that satisfies $N_{B}=1$ ) enclosed in $\mathcal{O}$ and reveals it during the first probes of the algorithm. Depending on the value of $\omega$ and probing strategy of the algorithm, three types of frames are possible: isosceles triangle with apex at $\nu_{\mathrm{B}}$ (for $0<\omega<\pi / 2$ ), quadrilateral or pentagon (for $\omega=\pi / 2$ ).

Let us start with the case when $0<\omega<\pi / 2$. The adversary creates an isosceles triangle with apex at $v_{\mathrm{B}}$ and base vertices $v_{1}$ and $v_{2}$. The triangle $\triangle\left(v_{\mathrm{B}}, v_{1}, v_{2}\right)$ contains $p ; v_{1}$ and $v_{2}$ are inside $A$, but not on the boundary; and the angle $\alpha$ at the apex of the triangle is such that $0<\alpha<\omega$ and $\alpha<\pi-2 \omega$. Refer to Figure 13.2. Notice that $v_{1}$ and $v_{2}$ are not fixed yet. The adversary can move them, depending on the the second query of the algorithm, as long as all the above constraints on the triangle hold.

The second valid probe of the algorithm is answered with respect to the $\omega$-cloud of the triangle, unless the direction of the probe coincides with one of its edges. In this case, the adversary slightly moves either $v_{1}$ or $v_{2}$, such that this probe will not confirm an edge. The adversary creates an $\omega$-cloud for the corrected triangle and answers the query according to the new $\omega$-cloud. This probe may reveal up to two new vertices of $\mathcal{O}$. If the current probe does not reveal $v_{1}$ or $v_{2}$, the adversary fixes both $v_{1}$ and $v_{2}$, but returns only information revealed by the probe to the algorithms. This concludes the initialization step for the case $0<\omega<\pi / 2$.

Let us explore the initialization step when $\omega=\pi / 2$. The adversary stops the first valid probe at any moment after the apex $q$ of the probe enters the interior of $\Psi$, but before it reaches $p$. Let $\left(q, H_{1}, H_{2}, p_{1}, p_{2}\right)$ be the outcome of the probe along $\vec{L}$ returned by the adversary, such that $H_{1}$ (respectively $\mathrm{H}_{2}$ ) makes a negative (respectively positive) angle of $\omega / 2$ with $\overrightarrow{\mathrm{L}} ; \mathrm{p}_{1} \neq \mathrm{q} ; \mathrm{p}_{2} \neq \mathrm{q}$; 
$p \in \overline{p_{1} p_{2}}$. In other words, on the first valid probe of the algorithm, the adversary reveals two vertices of $\mathcal{O}$. Refer to Figure 13.3a.

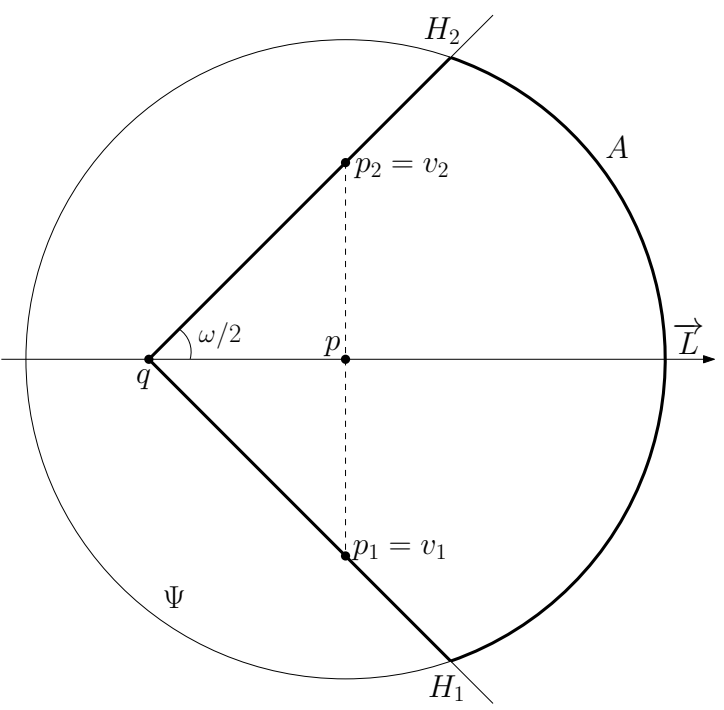

(a) The adversary reveals two vertices $v_{1}$ and $v_{2}$ of $\mathcal{O}$ on the first valid probe of the algorithm.

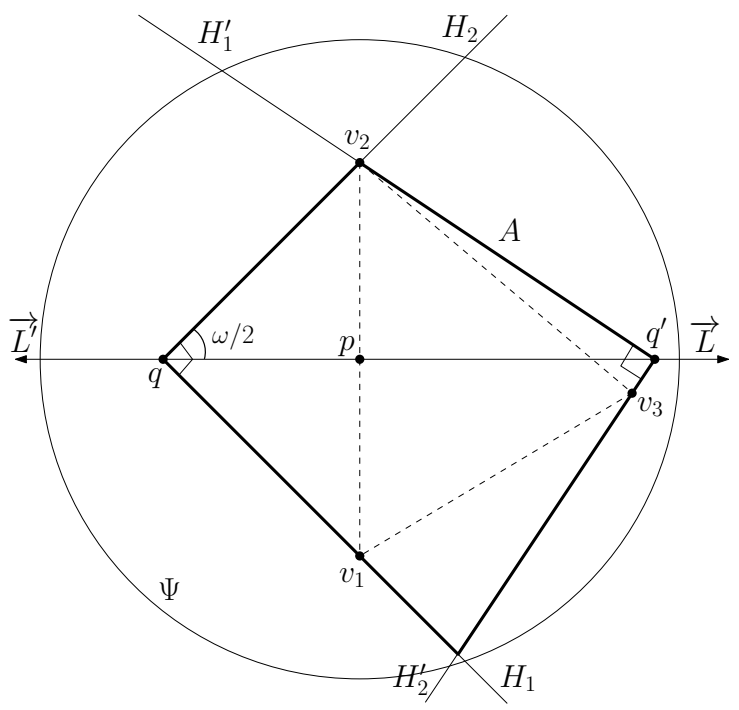

(b) The second valid probe of the algorithms is shot along the same line as its first valid probe but in the opposite direction.

Figure 13.3: Initialization step for $\omega=\pi / 2$. The closed curve $A$ is highlighted in bold.

Let $\overrightarrow{L^{\prime}}$ be the direction of the second valid probe of the algorithm. Three cases are possible:

1. If $v_{1}, v_{2} \in \overrightarrow{\mathrm{L}^{\prime}}$, then assume $\overrightarrow{\mathrm{L}^{\prime}}$ intersects $v_{1}$ before it intersects $v_{2}$ (the other case is symmetrical). Let $\left(q^{\prime}, H_{1}^{\prime}, H_{2}^{\prime}, p_{1}^{\prime}, p_{2}^{\prime}\right)$ be the outcome of the probe along $\overrightarrow{L^{\prime}}$ returned by the adversary, such that $\mathrm{q}^{\prime}=\mathrm{p}_{1}^{\prime}=\mathrm{p}_{2}^{\prime}=v_{1}, \mathrm{H}_{1}^{\prime}$ (respectively $\mathrm{H}_{2}^{\prime}$ ) makes a negative (respectively positive) angle of $\omega / 2$ with $\overrightarrow{L^{\prime}}$. In other words, the adversary declares the already revealed vertex $v_{1}$ as a narrow vertex of $\mathcal{O}$ and positions the arms of the second probe such that they are not coincide with the line segment $\overline{v_{1} v_{2}}$.

2. Else, if $\overrightarrow{L^{\prime}} \neq \overleftarrow{L}$, return the already discovered vertices $v_{1}$ and $v_{2}$ of $\mathcal{O}$. In other words, the adversary constructs the $\omega$-cloud of the only vertices $v_{1}$ and $v_{2}$ and returns the outcome of the probe along the given $\overrightarrow{\mathrm{L}^{\prime}}$ on this cloud. Assume, that the apex of the second probe is closer to $v_{2}$ than to $v_{1}$ (the other case is symmetrical). The adversary marks $v_{1}$ as a narrow vertex (the algorithm may correctly guess this fact).

3. If $\overrightarrow{\mathrm{L}^{\prime}}=\overleftarrow{\mathrm{L}}\left(\overrightarrow{\mathrm{L}^{\prime}}\right.$ coincides with $\overrightarrow{\mathrm{L}}$ but has the opposite direction), then the adversary reveals the new vertex $v_{3}$ of $\mathcal{O}$ and returns the following outcome: $\left(\mathrm{q}^{\prime}, \mathrm{H}_{1}^{\prime}, \mathrm{H}_{2}^{\prime}, \mathrm{p}_{1}^{\prime}, \mathrm{p}_{2}^{\prime}\right)$, where $\mathrm{p}_{1}^{\prime}=v_{2}$; $\mathrm{p}_{2}^{\prime}=v_{3}, \mathrm{q}^{\prime}$ and $v_{3}$ are close to each other, but $\mathrm{q}^{\prime} \neq v_{3} ;|\mathrm{qp}|<\left|\mathrm{pq}^{\prime}\right|$ and $\left|\mathrm{pq} \mathrm{q}^{\prime}\right|$ is smaller then the radius of $\Psi$. Refer to Figure 13.3b.

Let $\overrightarrow{L^{\prime \prime}}$ be the direction on the third valid probe of the algorithm. The answer of the adversary depends also on the direction of the previous probe $\overrightarrow{\mathrm{L}^{\prime}}$.

For the first two cases of $\overrightarrow{\mathrm{L}^{\prime}}$, the adversary defines two vertices: $v_{3}$ and $v_{4}$ (interior to $A$ but not on the boundary), such that three of the internal angles of the resulting quadrilateral $\left\{v_{1}, v_{3}, v_{2}, v_{4}\right\}$ are strictly bigger than $\pi / 2$ and the fourth internal angle, rooted at the narrow vertex, is strictly smaller than $\pi / 2$. Moreover, none of the edges of the quadrilateral is contained within $\overrightarrow{L^{\prime \prime}}$. Note that $v_{3}$ and $v_{4}$ 
become fixed after the adversary returns the outcome of the third valid probe of the algorithm (even if that probe does not discover one or both of them). Note also that there are infinitely many possibilities for the adversary to choose $v_{3}$ and $v_{4}$ to satisfy the above conditions. Refer to Lemma 11.5.

Let us explore the third case of $\overrightarrow{\mathrm{L}^{\prime}}$. Note that in this case, the algorithm already discovered three vertices of $\mathcal{O}: v_{1}, v_{2}$ and $v_{3}$. Refer to Figure 13.3b.

- If $\overrightarrow{L^{\prime \prime}}$ coincides with one of the edges of the triangle $\triangle\left(v_{1}, v_{2}, v_{3}\right)$, the adversary declares the vertex of entrance $\vec{L}^{\prime \prime}$ into $A$ as a narrow vertex and places the arms such that they maximize $A$ and do not touch other vertices of $\mathcal{O}$. For example, if $\overrightarrow{L^{\prime \prime}}$ contains $\overline{v_{1} v_{2}}$ and is directed from $v_{1}$ to $v_{2}$, the outcome of the probe $\left(\mathrm{q}^{\prime \prime}, \mathrm{H}_{1}^{\prime \prime}, \mathrm{H}_{2}^{\prime \prime}, \mathrm{p}_{1}^{\prime \prime}, \mathrm{p}_{2}^{\prime \prime}\right)$ is as follows: $\mathrm{q}^{\prime \prime}=\mathrm{p}_{1}^{\prime \prime}=\mathrm{p}_{2}^{\prime \prime}=v_{1} ; v_{2}, v_{3} \notin \mathrm{H}_{1}^{\prime \prime}$ and $v_{2}, v_{3} \notin \mathrm{H}_{2}^{\prime \prime}$.

- Otherwise, the adversary creates an $\omega$-cloud of the triangle $\Delta\left(v_{1}, v_{2}, v_{3}\right)$ and answers the query of the algorithm according to this cloud. In other words, the probe does not reveal any new information about $\mathcal{O}$, but may have changed the shape of $A$.

To finalize the initialization step and to create a frame for $\mathcal{O}$ with exactly one narrow vertex, the adversary creates one new vertex $v_{4}$ in the interior of $A$, such that the convex quadrilateral $\left\{v_{1}, v_{2}, v_{3}, v_{4}\right\}$ has exactly one acute angle. It is always possible in the case when $\overrightarrow{L^{\prime \prime}}$ contains edge of the triangle $\triangle\left(v_{1}, v_{2}, v_{3}\right)$. Unfortunately, it is not always true for general $\overrightarrow{\mathrm{L}}$. The adversary may require one more vertex $v_{5}$ (in the interior of $A$ ), such that the resulting convex pentagon $\left\{v_{1}, v_{2}, v_{3}, v_{4}, v_{5}\right\}$ has exactly one acute angle (and $v_{4}$ and $v_{5}$ are not adjacent on the boundary of the pentagon). During the following probes the algorithm may not discover $v_{4}$ or $v_{5}$. As soon as discovered part of $\mathcal{O}$ satisfies frame constraints (frame is a polygon with exactly one acute angle), the not discovered helping vertices can be discarded. Thus, during the second stage of his strategy, the adversary places a new vertex in a way that the internal angles of $\mathcal{O}$ are maximized (as long as the internal angle corresponding to the narrow vertex is smaller than $\omega$ ). Notice that the vertices $v_{4}$ and $v_{5}$ can be discovered by one probe of the algorithm. The vertex at the acute angle of the pentagon or quadrilateral frame is marked as a narrow vertex (whether or not the algorithm discovered this fact). This concludes the initialization stage for $\mathrm{N}_{\mathrm{B}}=1$ and $0<\omega \leqslant \pi / 2$.

The rest of the adversary's strategy is similar to the one described in the Section 12.3. The only difference is that when a new vertex is inserted between two existing ones (one of which is a narrow vertex), the angle at the narrow vertex should stay smaller than $\omega$. These precautions are taken since the narrow vertex may have been discovered by the algorithm but not necessarily identified as a narrow vertex, and thus the internal angle at the corresponding vertex of $A$ (where the boundary of $A$ touches the narrow vertex) can be bigger than $\omega$.

We analyze the number of probes spent by the algorithm in the following theorem.

THEOREM 13.2 (Lower Bound $N_{B}=1$ ). Let an $\omega$-wedge with $0<\omega \leqslant \pi / 2$ be given. For every algorithm, there exists a convex polygon $\mathcal{O}$ with one narrow vertex, such that $2 \mathrm{n}-1 \mathrm{w}$-probes are required to determine its shape.

Proof. If $0<\omega<\pi / 2$, the initialization step results in 3 vertices of $\mathcal{O}$ (one of which is a narrow vertex) and requires at least 2 probes. The rest of the polygon is reconstructed in at lest $2 n-3$ probes. (Refer to the Section 12.3.) Thus, in total, $2 n-1$ probes are required to reconstruct $\mathcal{O}$.

If $\omega=\pi / 2$, the algorithm spends at least one probe to discover the first 2 vertices. Depending on the strategy of the algorithm, the initialization step terminates after at least one more probe, that returns no new vertices or edges; or after at least 2 additional probes that result in one new vertex. Notice that because of the constraints induced by the frame, at most one of the consecutive probes may return 2 new pieces of information. The rest of $\mathcal{O}$ requires at least one probe per edge and at least one probe per vertex. (Refer to the Section 12.3). This results in at least $2 n-1$ probes and proves the following theorem. 


\subsubsection{Lower Bound for Polygons with Two or Three Narrow Vertices}

For $N_{B} \geqslant 2$ the adversary should provide the algorithm with a small real number $\varepsilon>0$. For every pair of narrow vertices of $\mathcal{O}: v_{\mathrm{B}_{1}}$ and $\nu_{\mathrm{B}_{2}}$, the following is true: if $\overline{\overline{\mathrm{B}}_{1} \nu_{\mathrm{B}_{2}}}$ is not an edge that belongs to $\mathcal{O}_{v_{B_{1}}} v_{\mathrm{B}_{2}}$, then there exists a vertex $v \in \mathcal{O}_{v_{\mathrm{B}_{1}} v_{\mathrm{B}_{2}}}$ such that $\angle\left(v_{\mathrm{B}_{2}}, v, v_{\mathrm{B}_{1}}\right) \leqslant \pi-\varepsilon$. This ensures that one of the angles $\angle\left(v, v_{\mathrm{B}_{1}}, v_{\mathrm{B}_{2}}\right)$ or $\angle\left(v_{\mathrm{B}_{1}}, v_{\mathrm{B}_{2}}, v\right)$ is at least $\varepsilon / 2$. Without $\varepsilon$, the polygon may not be reconstructed.

Let us describe the adversary's strategy for convex polygons with exactly two narrow vertices. The adversary chooses $\varepsilon=\omega / 10$, defines a circle $\Psi$, a point $p$ as a center of $\Psi$ and reveals all this information to the algorithm. It then chooses $n \geqslant 3$, sets $N_{B}=2$, initialises $A$ to be $\Psi$ and sets flag $=$ false.

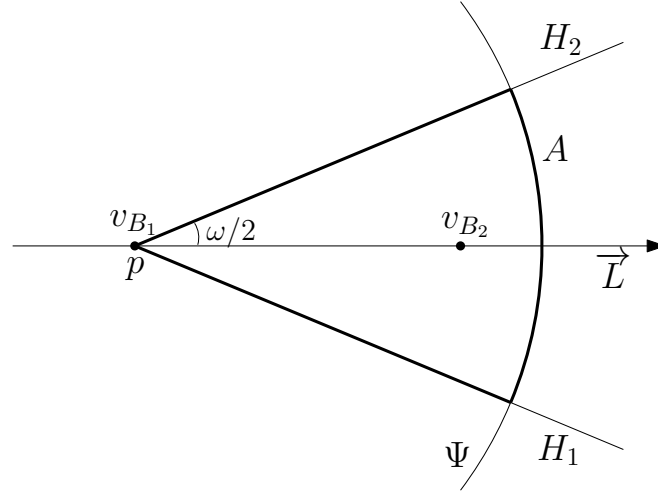

(a) The apex of the first valid probe of the algorithms coincides with $p$.

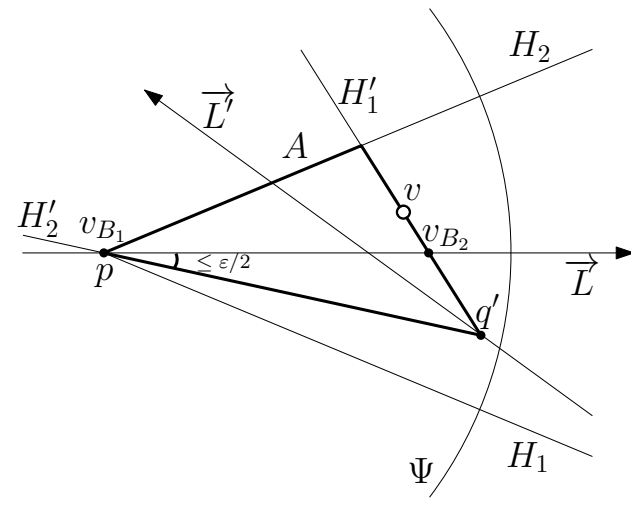

(b) Possible result of the second valid probe. For the sake of demonstration the size of the angle $\angle\left(q^{\prime}, p, v_{B_{2}}\right)$ is exaggerated. The vertex $v$ is the possible new position for $v_{\mathrm{B}_{2}}$.

Figure 13.4: Initialization step for $N_{B}=2$. The closed curve $A$ is highlighted in bold.

Let $\vec{L}$ be the direction of the first valid probe of the algorithm such that $p \in \overrightarrow{\mathrm{L}}$. Otherwise, we let this probe miss the object and perform updates to our working space and $A$ as described in Section 12.3. The adversary stops the probe when its apex coincides with $p$. Let $\left(q, H_{1}, H_{2}, p_{1}, p_{2}\right)$ be the outcome of the probe along $\vec{L}$ returned by the adversary, such that: $q=p=v_{B}=p_{1}=p_{2}, H_{1}$ (respectively $\mathrm{H}_{2}$ ) makes a negative (respectively positive) angle of $\omega / 2$ with $\overrightarrow{\mathrm{L}}$. Refer to Figure 13.4a. Notice that the shape of the polygon with two narrow vertices can be skinny. To ensure $p \in \mathcal{O}$, we let $v_{\mathrm{B} 1}=\mathrm{p}$.

We create another narrow vertex $v_{\mathrm{B} 2}$. Initially, the adversary places it such that $v_{\mathrm{B} 2} \in \overrightarrow{\mathrm{L}}$ and $v_{\mathrm{B} 2} \in A$, but not on the boundary of $A$, which also ensures that $v_{\mathrm{B} 1} \neq v_{\mathrm{B} 2}$. Let $\overrightarrow{\mathrm{L}^{\prime}}$ be the direction of the second valid probe made by the algorithm, such that $\overrightarrow{\mathrm{L}^{\prime}}$ intersects the line segment $\overline{v_{\mathrm{B}_{1}} v_{\mathrm{B}_{2}}}$. Let $\left(\mathrm{q}^{\prime}, \mathrm{H}_{1}^{\prime}, \mathrm{H}_{2}^{\prime}, \mathrm{p}_{1}^{\prime}, \mathrm{p}_{2}^{\prime}\right)$ be the outcome of the probe on the polygon $\left\{v_{\mathrm{B} 1}, v_{\mathrm{B} 2}\right\}$. If $\overrightarrow{\mathrm{L}^{\prime}}$ does not intersect $\overline{v_{B_{1}} v_{\mathrm{B}_{2}}}$, then the adversary does the following: declares the probe as not valid (meaning, the probe missed the object), shrinks its workspace such that the updated $A$ does not have common points with $\overrightarrow{\mathrm{L}^{\prime}}$, and creates $v_{\mathrm{B} 2}$ as described earlier.

- If $\mathrm{q}^{\prime}=v_{\mathrm{B}_{2}}$ or $\mathrm{q}^{\prime}=v_{\mathrm{B}_{1}}$, we rotate the $\omega$-wedge around its apex such that $\overrightarrow{\mathrm{L}}$ bisects the wedge and $\mathcal{O}$ stays inside the wedge. The adversary fixes $\nu_{\mathrm{B}_{2}}$ on its current position, marks it as a narrow vertex and returns the result of the second probe to the algorithm. 
- Otherwise, if none of the arms $\mathrm{H}_{1}^{\prime}$ or $\mathrm{H}_{2}^{\prime}$ creates an angle of $\varepsilon / 2$ or smaller with $\overrightarrow{\mathrm{L}}$, then fix $v_{\mathrm{B}_{2}}$ on its current position and return the output of the second probe to the algorithm. The adversary marks $v_{\mathrm{B}_{2}}$ as a narrow vertex (notice that in this case, the algorithm did not discover this fact, but may correctly guess it).

- Otherwise, if $\mathrm{H}_{1}^{\prime}$ or $\mathrm{H}_{2}^{\prime}$ creates an angle of $\varepsilon / 2$ or smaller with $\overrightarrow{\mathrm{L}}$, then two cases are possible:

1. If the angle between $\vec{L}$ and $\overrightarrow{L^{\prime}}$ is bigger than $\pi / 2$ (refer to Figure 13.4b) the adversary moves $v_{\mathrm{B}_{2}}$ along the supporting arm of the second probe away from $\mathrm{q}^{\prime}$, such that $\angle\left(\mathrm{q}^{\prime}, \mathrm{p}, v_{\mathrm{B}_{2}}\right)>\varepsilon / 2$ and the angles between the line segment $\overline{\nu_{\mathrm{B}_{1}} \nu_{\mathrm{B}_{2}}}$ with $\mathrm{H}_{1}$ and $\mathrm{H}_{2}$ are bigger than $\varepsilon / 2$. (Consider, for example, Figure $13.4 \mathrm{~b}$. The possible new position for $\nu_{\mathrm{B}_{2}}$ is marked by $v$.)

2. If the angle between $\overrightarrow{\mathrm{L}}$ and $\overrightarrow{\mathrm{L}^{\prime}}$ is smaller than $\pi / 2$, we consider the two possible values of flag:

- If flag $=$ false, the adversary declares the current probe as non valid, shrinks its working space, updates $A$, creates $\nu_{\mathrm{B}_{2}}$ and sets flag $=$ true.

- If flag $=$ true, the adversary fixes $v_{\mathrm{B}_{2}}$ on its current position and returns the output of the current probe to the algorithm.

This concludes the initialization step.

The other stages of the adversary's strategy are similar to those used for $N_{B}=0$ (refer to Section 12.3) and $N_{B}=1$ (refer to the beginning of the current section). The only difference is due to the use of $\varepsilon$.

As in the previous strategies, during the second stage, we answer the probes with already revealed vertices, unless the direction of the probe contains two known vertices and the probe does not enter $\mathcal{O}$ through a narrow vertex. In this case, we reveal one new vertex provided that the number of already revealed vertices is smaller than $n-2$. Similarly to the strategy used for $N_{B}=1$, all newly created vertices in this stage should not change the number of narrow vertices of $\mathrm{Q} \subseteq \mathcal{O}$.

The adversary proceeds to the third stage of its strategy when the algorithm knows $n-1$ vertices of $\mathcal{O}$. During this stage the adversary confirms edges (without revealing any new vertices), until $n-2$ edges are confirmed. Every probe of the algorithm is answered with already revealed vertices of $\mathcal{O}$, unless the direction of the probe $\vec{L}$ contains two vertices adjacent on $A$ such that $\vec{L}$ does not enter $\mathcal{O}$ through a narrow vertex. In this case, an edge between the pair of vertices is confirmed.

During the final, fourth stage of the strategy, the last unknown vertex is revealed to the algorithm and its two adjacent edges are confirmed.

Without knowing $\varepsilon$, the algorithm may not be able to reconstruct $\mathcal{O}$ with $\mathrm{N}_{B} \geqslant 2$ precisely. Let $\overrightarrow{\mathrm{L}_{v_{B_{1}} v_{B_{2}}}}$ be the line through a pair of narrow vertices $v_{\mathrm{B}_{1}}$ and $v_{\mathrm{B}_{2}}$, directed from $v_{\mathrm{B}_{1}}$ to $v_{\mathrm{B}_{2}}$. Suppose that there are no revealed vertices of $\mathcal{O}$ to the left of $\overrightarrow{\mathrm{L}_{\mathrm{B}_{1} v_{\mathrm{B}_{2}}}}$. Let $\mathrm{F}$ be a feasible region of $\mathrm{Q} \subseteq \mathcal{O}$ to the left of $\overrightarrow{\mathrm{L}_{\mathrm{B}_{1}} v_{\mathrm{B}_{2}}}$. Assume that the probe of the algorithm stops when $v_{\mathrm{B}_{1}} \in \mathrm{H}_{1}$ and $v_{\mathrm{B}_{2}} \in \mathrm{H}_{2}$ (notice, that the apex of the probe is to the left of $\overrightarrow{\mathrm{L}_{v_{B_{1}} v_{B_{2}}}}$ ). If the angle between $\mathrm{H}_{2}$ (respectively, $\mathrm{H}_{1}$ ) and $\overrightarrow{\mathrm{L}_{\mathrm{B}_{1}} v_{\mathrm{B}_{2}}}$ is smaller than $\varepsilon / 2$ and the angle at $v_{\mathrm{B}_{1}}$ (respectively, $v_{\mathrm{B}_{2}}$ ) of $\mathrm{F}$ is smaller than $\varepsilon / 2$, then:

1. If we are in the second stage of the strategy, one new vertex $v$ is created in F (but not on its boundary). The adversary makes the probe touch $v$ and returns the output to the algorithm. Notice that in the second stage, $\mathcal{O}$ has no confirmed edges.

2. If we are in the third stage, the adversary outputs the result as it is and the algorithm can interpret it as an edge between $v_{\mathrm{B}_{1}}$ and $v_{\mathrm{B}_{2}}$. In this case, $\mathrm{F}$ is the line segment $\overline{\mathrm{v}_{\mathrm{B}_{1}} v_{\mathrm{B}_{2}}}$. 
3. If we are in the fourth stage and the algorithm did not reveal the last $n$-th vertex of $\mathcal{O}$, the adversary proceeds similarly to Step 1. If the algorithm knows all the vertices but did not confirm all the edges, we proceed according to Step 2.

We conclude the adversary's strategy with the case when $N_{B}=3$. Notice that this case can happen only when $\pi / 3<\omega \leqslant \pi / 2$. The last three stages of the strategy are identical to the case when $N_{B}=2$, so we describe in short the initialization step only.

The adversary chooses $\varepsilon=\frac{1}{10}(\omega-\pi / 3)$, defines a circle $\Psi$, a point $p$ as a center of $\Psi$ and reveals all this information to the algorithm. It then chooses $n \geqslant 3$, sets $N_{B}=3$ and initialises $A$ to be $\Psi$.

On the first valid probe of the algorithm, whose direction $\vec{L}$ contains $p$, the adversary returns two vertices of $\mathcal{O}: v_{B_{1}}$ and $v_{B_{2}}$. Let $\left(q, H_{1}, H_{2}, p_{1}, p_{2}\right)$ be the outcome of the probe along $\vec{L}$ returned by the adversary, such that: $p_{1}=v_{B_{1}}, p_{2}=v_{B_{2}}, q \neq p_{1}, q \neq p_{1},\left|q p_{1}\right|=\left|q p_{2}\right|, H_{1}$ (respectively $H_{2}$ ) makes a negative (respectively positive) angle of $\omega / 2$ with $\vec{L}$. Refer to Figure 13.5. The adversary marks $v_{B_{1}}$ and $v_{\mathrm{B}_{2}}$ as narrow vertices.

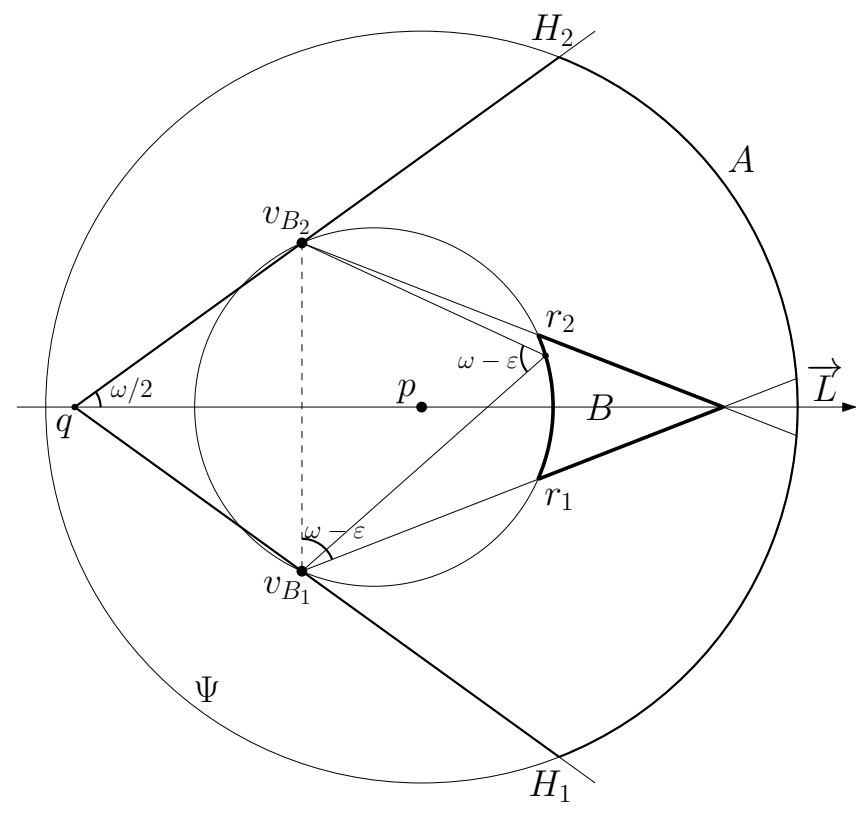

Figure 13.5: Initialization step for $N_{B}=3$. The closed curve $A$ is highlighted in bold. The third narrow vertex $v_{\mathrm{B}_{3}}$ can be positioned inside the region $B$, marked in extra-bold.

Let $r_{1}$ (respectively, $r_{2}$ ) be a ray emanating from $v_{\mathrm{B}_{1}}$ (respectively, $v_{\mathrm{B}_{2}}$ ) and making a negative (respectively, positive) angle of $\omega-\varepsilon$ with the line segment $\overline{v_{B_{1}} v_{\mathrm{B}_{2}}}$. Let $p^{\prime}$ be a point interior to the $\omega$-wedge of the first valid probe, such that $\angle\left(v_{\mathrm{B}_{2}}, \mathrm{p}^{\prime}, v_{\mathrm{B}_{1}}\right)=\omega-\varepsilon$, and let $C$ be a disk defined by $v_{\mathrm{B}_{1}}$, $v_{\mathrm{B}_{2}}$ and $\mathrm{p}^{\prime}$. Let $\mathrm{H}_{\mathrm{r}_{1}}$ (respectively, $\mathrm{H}_{\mathrm{r}_{2}}$ ) be a half-plane containing $\mathrm{r}_{1}$ (respectively, $\mathrm{r}_{2}$ ) on its boundary and containing $q$. Let $\mathrm{H}^{\prime}$ be a half-plane containing $\overline{\nu_{\mathrm{B}_{1}} \nu_{\mathrm{B}_{2}}}$ on its boundary and not containing $\mathrm{q}$. The region $B$, where the third narrow vertex $v_{B_{3}}$ can be positioned, is defined as $\Psi \cap \mathrm{H}_{\mathrm{r}_{1}} \cap \mathrm{H}_{\mathrm{r}_{2}} \cap \mathrm{H}^{\prime} \backslash \mathrm{C}$. Refer to Figure 13.5.

The adversary stops the first valid probe in such a way that: $B$ is non-empty; $v_{B_{1}}$ and $v_{B_{2}}$ are interior to $\Psi$; and for every point $\mathrm{t} \in \mathrm{B}$, the triangle $\triangle\left(v_{\mathrm{B}_{1}}, v_{\mathrm{B}_{2}}, \mathrm{t}\right)$ contains $\mathrm{p}$.

Let $L^{\prime}$ be the direction of the next valid probe of the algorithm. The vertex $\nu_{\mathrm{B}_{3}}$ is created and revealed when it is possible to position the $\omega$-wedge of the probe along $L^{\prime}$ such that one of its arms, or the apex itself, intersects $B$. The vertex $v_{\mathrm{B}_{3}}$ should be created such that none of the arms of the probe makes an angle of $\varepsilon / 2$ or smaller with the line segment $\overline{\nu_{\mathrm{B}_{1}} \nu_{\mathrm{B}_{2}}}, \overline{\nu_{\mathrm{B}_{2}} \nu_{\mathrm{B}_{3}}}$ or $\overline{{\mathrm{B}_{1}}_{1} \nu_{\mathrm{B}_{3}}}$. Since $v_{\mathrm{B}_{1}}$ and $v_{\mathrm{B}_{2}}$ 
are narrow vertices, $v_{\mathrm{B}_{3}}$ cannot be revealed simultaneously (via the same probe) with a new vertex or an edge.

Notice that the region $\mathrm{B}$ can shrink as a result of the vertex $v$ being revealed between $v_{\mathrm{B}_{1}}$ and $v_{\mathrm{B}_{2}}$ on the boundary of $\mathcal{O}$ in a counter-clockwise direction from $v_{\mathrm{B}_{2}}$ to $v_{\mathrm{B}_{1}}$. We prevent $\mathrm{B}$ from disappearing by positioning $v$ such that $\pi-2 \varepsilon \leqslant \angle\left(\nu_{\mathrm{B}_{1}}, \nu, \nu_{\mathrm{B}_{2}}\right) \leqslant \pi-\varepsilon$.

THEOREM 13.3 (Lower Bound $\mathrm{N}_{\mathrm{B}}=2, \mathrm{~N}_{\mathrm{B}}=3$ ). Given $\omega$-wedge $(0<\omega \leqslant \pi / 2)$, convex polygon $\mathcal{O}$, and a real number $\epsilon>0$ (such that for every pair of narrow vertices of $\mathcal{O}: v_{\mathrm{B}_{1}}$ and $\nu_{\mathrm{B}_{2}}$, if $\overline{v_{\mathrm{B}_{1}} \nu_{\mathrm{B}_{2}}}$ is not an edge

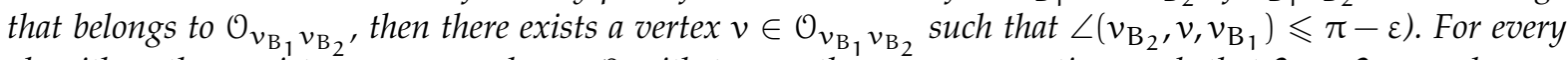
algorithm, there exists a convex polygon $\mathcal{O}$ with two or three narrow vertices such that $2 \mathrm{n}+2 \mathrm{w}$-probes are required to determine its shape.

Proof. Suppose that $\mathrm{N}_{\mathrm{B}}=3$. During the initialization step, at least one probe is used and two vertices are revealed. One of the subsequent probes reveals $v_{\mathrm{B}_{3}}$ and no additional information. The algorithm wastes at least one probe per every pair of narrow vertices, which results in at least 3 probes and no new information. The rest of the probes return each at most one new piece of information about $\mathcal{O}$, making it at least $2 n-3$ probes. Thus, a total of $2 n+2$ probes are necessary to reconstruct $\mathcal{O}$.

The analysis of the strategy for polygons with $\mathrm{N}_{\mathrm{B}}=2$ is similar. The initialization step requires at least two probes and results in two vertices of $\mathcal{O}$. At least two probes bring no new information due to the usage of $\varepsilon$. Not less than $2 n-2$ additional probes are required to reveal $n-2$ vertices and to confirm $n$ edges. This adds up to at least $2 n+2$ probes. 


\section{CONCLUSION AND FUTURE WORK}

\section{4}

In this thesis we presented an $\omega$-wedge device together with optimal probing algorithms for convex polygons with no acute angles or when at most one angle is smaller then $\omega$. When the number of such angles is more than one (possibly only two or three), the performance is weaker, yet we show it to be almost optimal. The main reason why our results are not tight is due to the fact that the number of bad angles is assumed a priori unknown. Knowing this extra information about polygon $\mathcal{O}$ beforehand would improve our strategy and bring down the upper bounds. In particular, Lemma 14.I and Observation 14.2 show that it is possible to detect that a particular vertex is narrow without specifically confirming this fact, that is, saving one probe. This means that we could save up to 3 probes (for polygons with exactly 3 narrow vertices), which would make our strategy optimal.

LEMma 14.1. Assume that we are given a convex polygon $\mathcal{O}$ with exactly 3 narrow vertices. Every valid $\omega$-probe of $\mathcal{O}$ touches a narrow vertex.

Proof. Assume to the contrary that the outcome $\left(\mathrm{q}, \mathrm{H}_{1}, \mathrm{H}_{2}, \mathrm{p}_{1}, \mathrm{p}_{2}\right)$ of the valid probe returns two vertices $p_{1}$ and $p_{2}$ such that none of them is a narrow vertex. Refer to Figure 14.1. By definition, a narrow vertex belongs to the $\omega$-cloud of $\mathcal{O}$, meaning that there is no arc of the $\omega$-cloud above the narrow vertex. Thus, there are no narrow vertices between $p_{1}$ and $p_{2}$ in a counter-clockwise direction from $p_{2}$ to $p_{1}$ around the boundary of $\mathcal{O}$.

Let $v_{\mathrm{B}_{1}}, v_{\mathrm{B}_{2}}$ and $v_{\mathrm{B}_{3}}$ be the three narrow vertices of $\mathcal{O}$. Let $v$ be an intersection of $\mathrm{H}_{2}$, and the line through $v_{\mathrm{B}_{1}}$ and $v_{\mathrm{B}_{2}}$; and let $u$ be an intersection of $\mathrm{H}_{1}$, and the line through $\nu_{\mathrm{B}_{1}}$ and $\nu_{\mathrm{B}_{3}}$. Consider the quadrilateral $\left\{q, u, v_{B_{1}}, v\right\}$. By definition, the internal angles of $\mathcal{O}$ corresponding to the narrow vertices are smaller or equal to $\omega$. Thus, the internal angles of the quadrilateral at $v$ and $u$ are strictly smaller than $\omega$. The angle at q equals $\omega$. Thus, the sum of the internal angles of the quadrilateral is smaller then $4 \omega$ and thus smaller then $2 \pi$, which is a contradiction.

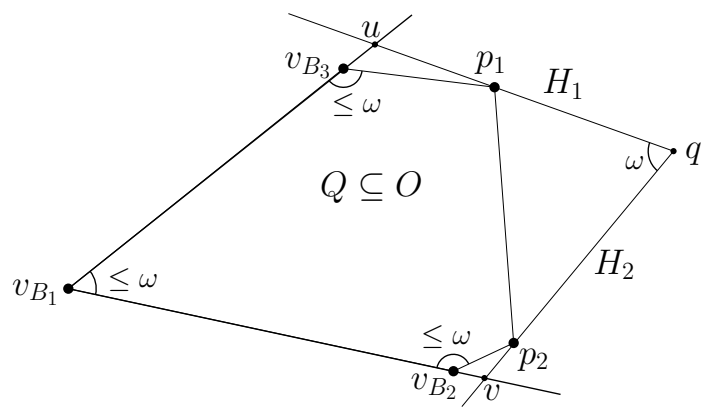

Figure 14.1: Illustration of the proof of Lemma 14.1. $\nu_{\mathrm{B}_{1}}, \nu_{\mathrm{B}_{2}}$ and $\nu_{\mathrm{B}_{3}}$ are narrow vertices.

The following observation follows from Lemma 14.1.

obSERVATION 14.2. Assume that we are given a convex polygon $\mathcal{O}$ with exactly 3 narrow vertices. Let (q, $\left.\mathrm{H}_{1}, \mathrm{H}_{2}, \mathrm{p}_{1}, \mathrm{u}\right)$ and $\left(\mathrm{q}^{\prime}, \mathrm{H}_{1}^{\prime}, \mathrm{H}_{2}^{\prime}, \mathrm{u}, \mathrm{p}_{2}\right)$ be two outcomes of two valid $\mathrm{\omega}$-probes of $\mathcal{O}$, respectively. Suppose that $\mathrm{q}$ and $\mathrm{q}^{\prime}$ belong to different arcs of $\mathrm{\omega}$-cloud of $\mathcal{O}$ and $\mathrm{p}_{1} \neq \mathrm{p}_{2}$. If $\angle\left(\mathrm{p}_{1}, \mathrm{u}, \mathrm{p}_{2}\right)>\omega$, then $\mathrm{p}_{1}$ and $\mathrm{p}_{2}$ are narrow vertices. Otherwise, if $\angle\left(\mathrm{p}_{1}, \mathrm{u}, \mathrm{p}_{2}\right) \leqslant \omega, \mathrm{u}$ is a narrow vertex.

Alternatively, we can take advantage of composite probing strategy that incorporates usage of up to 3 finger probes. Whenever we have a pair of narrow vertices $v_{\mathrm{B}_{1}}, v_{\mathrm{B}_{2}}$ (with no additional information 
discovered between them on the boundary of $\mathcal{O}$ ), we can shoot a finger probe orthogonally to $\overline{v_{\mathrm{B}_{1}} \nu_{\mathrm{B}_{2}}}$. For convex polygons with two or three narrow vertices we will no longer require the help of $\varepsilon$ and no probes will be wasted on every pair of narrow vertices. This will result in optimal strategy without the advantage of knowing number of narrow vertices beforehand.

We would like to explore different models of $\omega$-probing. For instance, one in which the outcome of a probe does not give us the contact points with the polygon. We know that $\mathcal{O}$ belongs to the interior of a valid $\omega$-probe. We are given the orientation of the arms $\mathrm{H}_{1}$ and $\mathrm{H}_{2}$ and we know that they touch $\mathcal{O}$, but we do not know the exact location of these contact points.

In our current model, we reconstruct $\mathcal{O}$ starting from within of $\mathcal{O}$. We form a polygon $Q$ by connecting known vertices of $\mathcal{O}$, so $Q$ completely resides inside $\mathcal{O}$. We expand $Q$ by adding newly discovered vertices of $\mathcal{O}$, so at the end of the reconstruction, $Q$ equals $\mathcal{O}$.

In the model we just described, we should use a different approach. The convex polygon $Q$ is defined as a boundary of an intersection of the circle $\Psi$ and all $\omega$-probes made so far, implying that $\mathcal{O}$ completely resides inside $\mathrm{Q}$. By performing additional probes, we cut away parts of $\mathrm{Q}$ so it shrinks closer to $\mathcal{O}$.

Another, interesting model of $\omega$-probing to explore would be one where the outcome of a probe is simply the apex.

This thesis investigates $\omega$-probing with small $\omega^{\prime}$ s: $0<\omega \leqslant \pi / 2$. It would be interesting to consider cases when $\pi / 2<\omega<\pi$. In the latter case, the structure of the $\omega$-cloud is more complicated, since we no longer have the fact that each arc participates in $\omega$-cloud at most once. 
Part III ART GALLERY LOCALIZATION 


\section{INTRODUCTION}

\section{5}

The art gallery problem was introduced in 1973 when Victor Klee asked how many guards are sufficient to guard the interior of a simple polygon having $n$ vertices. Although it has been shown by Chvátal that $\lfloor n / 3\rfloor$ guards are always sufficient and sometimes necessary [13], and such a set of guards can be computed easily [20], such solutions are usually far from optimal in terms of minimizing the number of guards for a particular input polygon. Moreover, it was shown that determining an optimal set of guards is NP-hard, even for simple polygons [32].

Trilateration is the process of determining absolute or relative locations of points by measurement of distances, using the geometry of the environment. In addition to its interest as a geometric problem, trilateration does have practical applications in surveying and navigation, including global positioning systems (GPS). Every GPS satellite transmits information about its position and the current time at regular intervals. These signals are intercepted by a GPS receiver, which calculates how far away each satellite is based on how long it took for the messages to arrive. GPS receivers take this information and use trilateration to calculate the user's location.

In our research we combine the art gallery problem with trilateration. We would like to address the problem of placing broadcast towers in a simple polygon $\mathrm{P}$ in order for a point in $\mathrm{P}$ (let us call it an agent) to locate itself. Towers can be defined as points which can transmit their coordinates together with a time stamp to other points in their visibility region. The agent receives messages from all the towers that belong to its visibility region. Given a message from the tower $t$, the agent can determine its distance to $t$. In our context, trilateration is the process, during which the agent can determine its absolute coordinates. Receiving a message from one tower only will not be sufficient for the agent to locate itself (unless the agent and the tower are at the the same location). In two-dimensional geometry, it is known that if a point lies on two circles, then the circle centers and the two radii provide sufficient information to narrow the possible locations down to two. Additional information may narrow the possibilities down to one unique location.

In relation to GPS systems, towers can be viewed as GPS satellites, while agents (query points interior to the polygon) can be compared to GPS receivers. Naturally, we would like to minimize the number of towers.

Let $P$ be a simple polygon in general position (no three vertices are collinear) having a total of $n$ vertices on its boundary (denoted by $\partial \mathrm{P}$ ). Note that $\partial \mathrm{P} \subset \mathrm{P}$. Two points $u, v \in \mathrm{P}$ are visible to each other if the segment $\overline{u v}$ is contained in P. We also say that $u$ sees $v$. Note that $\overline{u v}$ may touch $\partial \mathrm{P}$ in one or more points. For $u \in P$, we let $V(u)$ denote the visibility polygon of $u$, as the set of all points $q \in P$ that are visible to $u$. Notice that $\mathrm{V}(\mathrm{u})$ is a star-shaped polygon contained in $\mathrm{P}$ and $\mathrm{u}$ belongs to its kernel (the set of points from which all of $\mathrm{V}(\mathrm{u})$ is visible).

Problem Definition: Let $\mathrm{T}$ be a set of points (called towers) in $\mathrm{P}$ satisfying the following properties. For any point $p \in P$ : for each $t \in T \cap V(p)$, the point $p$ receives the coordinates of $t$ and can compute the Euclidean distance between $t$ and $p$, denoted $d(t, p)$. From this information, $p$ can determine its coordinates. We consider the following problems:

1. Design an algorithm that, on any input polygon $\mathrm{P}$ in general position, computes a "small" set $\mathrm{T}$ of towers.

2. Design a localization algorithm.

We show how to compute such a set $T$ of size at most $\lfloor 2 n / 3\rfloor$ by using the polygon partition method introduced by Tóth [44]. Tóth showed that any simple polygon with $n$ vertices can be guarded by 
$\lfloor n / 3\rfloor$ point guards whose range of vision is $180^{\circ}$. However, Tóth assumed that his partition method creates subpolygons whose vertices are in general position. We show how to adapt his method to polygons whose vertices are not in general position and how to use it with respect to our problem. It is important to notice that we assume that the input polygon is in general position, while non-general position may occur for subpolygons of the partition. We show that each $180^{\circ}$-guard $\mathrm{g}$ can be replaced with a pair of towers close to $\mathrm{g}$. We embed the orientation of the $180^{\circ}$-guard into the coordinates of the towers. That is, we specify to which side of the line through the pair of towers their primary localization region resides. We call it the parity trick. The localization algorithm is allowed to use this information.

Our interest in this problem started with the paper by Dippel and Sundaram [17] presented at the Canadian Conference on Computational Geometry in 2015. They provide the first non-trivial bounds on agent localization in simple polygons, by showing that $[8 n / 9\rfloor$ towers suffice for any non-degenerate polygon of $n$ vertices, and present an $O(n \log n)$ algorithm for the corresponding placement. Their approach is to decompose the polygon into at most $\lfloor n / 3\rfloor$ fans. A polygon $P^{\prime}$ is a fan if there exist a vertex $u$, such that for every other vertex $v$ not adjacent to $u, \overline{u v}$ is a diagonal of $P^{\prime}$; the vertex $u$ is called the center of the fan. In each fan with fewer than 4 triangles Dippel and Sundaram position a pair of towers on an edge of the fan; every fan with 4 or more triangles receives a triple of towers in its kernel. In a classical trilateration, the algorithm for locating an agent knows the coordinates of the towers that can see $p$ together with distances between $p$ and the corresponding towers. However, the localization algorithm presented in [17] requires a lot of additional information, such as a map of the polygon, the decomposition into fans, the coordinates of all towers, and even the list of towers that do not see the agent.

Our localization algorithm has no information about P. It receives as an input only the coordinates of the towers that can see $p$ together with their distances to $p$. In addition our algorithm is empowered by the knowledge of the parity trick. When only a pair $t_{1}, t_{2}$ of towers can see $p$ then the coordinates of the towers together with the distances $d\left(t_{1}, p\right)$ and $d\left(t_{2}, p\right)$ provide sufficient information to narrow the possible locations of $p$ down to two. Refer to Figures 16.1b,16.1c. Those two locations are reflections of each other over the line through $t_{1}$ and $t_{2}$. In this situation our localization algorithm uses the parity trick. It calculates the distance between the two towers and judging by the parity of this number decides which of the two possible locations is the correct position of $p$.

We show how to position at most $\lfloor 2 n / 3\rfloor$ towers inside $P$ which is an improvement over the previous upper bound in [17]. We also show that $\lfloor 2 \mathrm{n} / 3\rfloor$ towers are sometimes necessary. The comb polygon from the original art gallery problem can be used to show a lower bound. Refer to Fig. 16.4a. No point in the comb can look into two different comb spikes. Thus we need at least two towers per spike to localize all of the points in its interior. In addition we need to know the parity trick. Or, alternatively, we need to know P, its exact location and orientation. We show in Theorem 16.3 that without any additional information (such as the parity trick or the complete knowledge about $\mathrm{P}$ including its partition) it is not possible to localize an agent in a simple $n$-gon (where $n=3 k+q$, for integer $k \geqslant 1$ and $q=0,1$ or 2 ) with less than $n-q$ towers.

In Chapter 16 we give basic definitions and present some properties and observations. Chapter 17 shows some of our modifications of the polygon partition given by Tóth [44] and its adaptation to our problem. In Chapter 18 we present a localization algorithm. 


\section{6}

Consider a point (an agent) $p$ in the interior of $P$, whose location is unknown. Let $C(x, r)$ denote the circle centered at a point $x$ with radius $r$. If only one tower $t$ can see $p$ then $p$ can be anywhere on $C(t, d) \cap V(t)$ (refer to Fig. 16.1a), which may not be enough to identify the location of $p$. By the map of $\mathrm{P}$ we denote the complete information about $\mathrm{P}$ including its shape, location and orientation. Notice that one must know the map of $P$ to calculate $V(t)$. If two towers $t_{1}$ and $t_{2}$ can see $p$ then the location of $p$ can be narrowed down to at most two points $C\left(t_{1}, d_{1}\right) \cap C\left(t_{2}, d_{2}\right) \cap V\left(t_{1}\right) \cap V\left(t_{2}\right)$. Refer to Fig. 16.1b. The ambiguity happens along the line through $t_{1}$ and $t_{2}$. To avoid this uncertainty we can place both towers on the same edge of P. Consider for example Fig. 16.1c where two towers are placed on the line segment $\operatorname{kernel}(\mathrm{P}) \cap \partial \mathrm{P}$. In this example, if the map of $\mathrm{P}$ is known (and thus we know $V\left(t_{1}\right)$ and $\left.V\left(t_{2}\right)\right)$ then the intersection $C\left(t_{1}, d_{1}\right) \cap C\left(t_{2}, d_{2}\right) \cap V\left(t_{1}\right) \cap V\left(t_{2}\right)$ is a single point (highlighted in red). Alternatively, if the map of $\mathrm{P}$ is unknown, we can place a triple of non-collinear towers in the kernel of $\mathrm{P}$ (highlighted in cyan on Fig. 16.1C) to localize any point interior to $\mathrm{P}$.

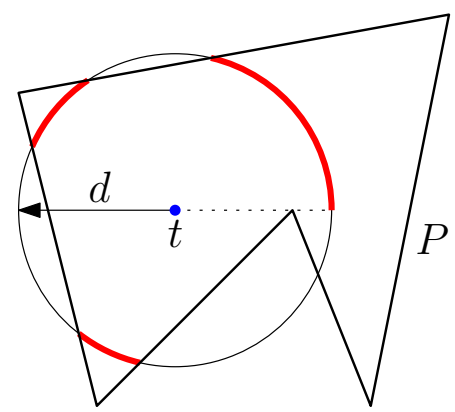

(a)

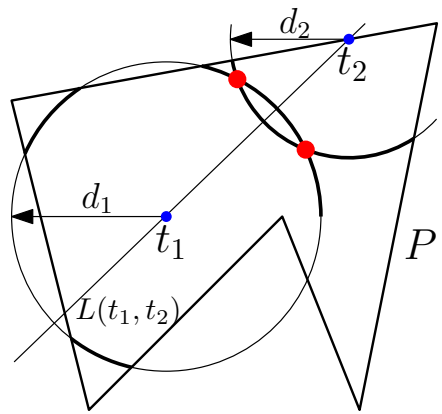

(b)

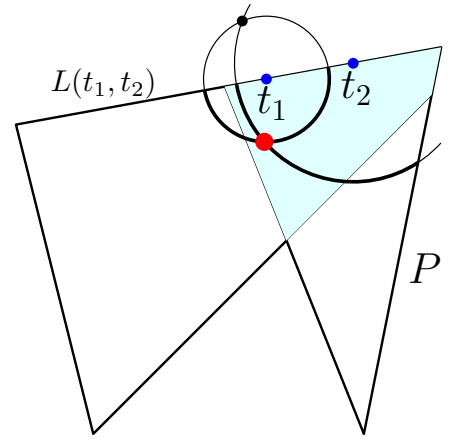

(c)

Figure 16.1: Trilateration example. (a) The point $p$ can be anywhere on $C(t, d) \cap V(t)$ which is highlighted in red. (b) Ambiguity along the line $L\left(t_{1}, t_{2}\right)$. (c) If the map of $P$ is known then the location of $p$ can be identified precisely. The kernel of $P$ is highlighted in cyan.

For a general simple polygon $\mathrm{P}$, we can partition it into star-shaped polygons $\mathrm{P}_{1}, \mathrm{P}_{2}, \ldots \mathrm{P}_{l}$ such that kernel $\left(P_{i}\right)$, for every $1 \leqslant i \leqslant l$, does not degenerate into a single point. In every $P_{i}(1 \leqslant i \leqslant l)$ we can position a pair of towers on a line segment in $\operatorname{kernel}\left(\mathrm{P}_{i}\right) \cap \partial \mathrm{P}_{i}$ (such that the towers belong to the same edge of $\left.P_{i}\right)$ or a triple of towers in $\operatorname{kernel}\left(P_{i}\right)$ if $\operatorname{kernel}\left(P_{i}\right) \cap \partial P_{i}$ is empty or contains a single point. Notice that a pair of towers positioned on the edge of $P_{i}$ will not necessarily be on the boundary of $P$. Thus, to localize an agent, it is not enough to know the map of $P$. We need to know more, for example, if in addition to the map of $\mathrm{P}$ we know the partition of $\mathrm{P}$ into star-shaped polygons and which pair of towers is responsible for which subpolygon then the agent can be localized.

In our solution we do not use this extra information or the map of P. Moreover, to get a tight bound of $\lfloor 2 n / 3\rfloor$ towers, we abstain from placing a triple of towers per subpolygon, since some polygons cannot be partitioned into less than $\lfloor n / 3\rfloor$ star-shaped subpolygons. The idea is to use a parity trick.

Parity trick: Let $\mathrm{L}(u, v)$ be the line through points $u$ and $v$. Let $\mathrm{L}(u, v)^{+}$denote the half plane to the left of $\mathrm{L}(u, v)$ (or above, if $\mathrm{L}$ is horizontal). Similarly, $\mathrm{L}(u, v)^{-}$denotes the half plane to the right (or below) of L. We embed information about the primary orientation of the pair of towers into their coordinates. If we want a pair $t_{1}, t_{2}$ of towers to be responsible for $L\left(t_{1}, t_{2}\right)^{+}$(respectively $\left.L\left(t_{1}, t_{2}\right)^{-}\right)$, 
then we position the towers at a distance which is a reduced rational number whose numerator is even (respectively odd). In this way, we specify on which side of $L\left(t_{1}, t_{2}\right)$ the primary localization region of $t_{1}$ and $t_{2}$ resides.

For this trick to work, we should partition $P$ into at most $\lfloor n / 3\rfloor$ star-shaped polygons $P_{1}, \ldots, P_{l}$ such that $\operatorname{kernel}\left(P_{i}\right) \cap \partial P_{i}$ is not a single point for every $1 \leqslant i \leqslant l$.

THEOREM 16.1 (Chvátal's Theorem [13]). Every triangulation of a polygon with $\mathrm{n}$ vertices can be partitioned into $m$ fans where $m \leqslant\lfloor n / 3\rfloor$.

The statement of the following theorem may seem trivial, but the proof, which we provide for completeness, is not that simple.

THEOREM 16.2. Any simple polygon $\mathrm{P}$ in general position with 3, 4 or 5 sides is star-shaped and its kernel contains a boundary segment that is not a single point.

Proof. Let $n$ be the number of vertices of P. By Theorem 16.1, $P$ can be partitioned into $\lfloor n / 3\rfloor=1$ fans (since $n=3,4$ or 5 ). Notice that a fan is star-shaped by definition, from which $P$ is star-shaped. The kernel of $\mathrm{P}$ is an intersection of $n$ half-planes defined by the edges of $\mathrm{P}$. Let $v$ be a fan center (a vertex of a fan that belongs to its kernel). There are two cases to consider: (1) the angle of $\mathrm{P}$ at $v$ is convex or (2) the angle of $P$ at $v$ is reflex.

1. The angle of $\mathrm{P}$ at $v$ is convex. Let $e$ be one of the edges of $\mathrm{P}$ adjacent to $v$. There exists a point $\mathrm{q} \in \mathrm{e}$ such that $\mathrm{q} \neq v$ and the line segment $\overline{\mathrm{q} v}$ belongs to the kernel of the fan. Assume to the contrary that such q does not exist. Thus the boundary line of one of the half-planes contains $v$ and it is not defined by an edge adjacent to $v$. It follows, that $v$ is collinear with two more vertices of $P$. This contradicts to the fact that $P$ is in general position.

2. The angle of $\mathrm{P}$ at $v$ is reflex. Let $v_{1}, v_{2}$ be two vertices of $\mathrm{P}$ adjacent to $v$. Refer to Fig. 16.2.

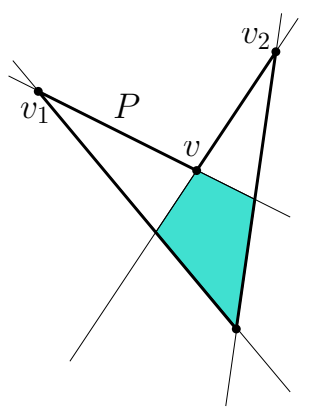

(a)

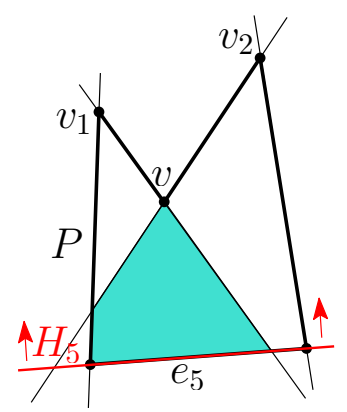

(b)

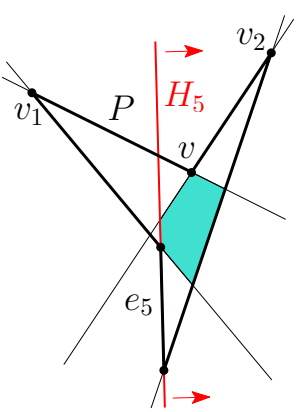

(c)

Figure 16.2: The kernel of $\mathrm{P}$ is highlighted in cyan.

The angles of $\mathrm{P}$ at $v_{1}$ and $v_{2}$ must be convex, otherwise $v$ is not in the kernel of $\mathrm{P}$. These angles should also be strictly bigger than 0 , otherwise $P$ is not in general position. Consider 4 halfplanes $\mathrm{H}_{i}(1 \leqslant i \leqslant 4)$ defined by the edges of $\mathrm{P}$ adjacent to $v, v_{1}$ and $v_{2}$. The intersection of $\mathrm{H}_{\mathrm{i}}$ $(1 \leqslant i \leqslant 4)$ is convex and can be either bounded or unbounded.

If $n=4$ then the intersection of $\mathrm{H}_{i}(1 \leqslant i \leqslant 4)$ is a convex quadrilateral with two sides contained in the edges of $\mathrm{P}$ (refer to Fig. 16.2a). It cannot degenerate into a point, a line segment or a triangle because $\mathrm{P}$ is in general position and the angle at $v$ is reflex.

If the intersection of $\mathrm{H}_{i}(1 \leqslant i \leqslant 4)$ is unbounded (refer to Fig. 16.2b) then $n=5$ and the fifth half-plane $\mathrm{H}_{5}$ (defined by the edge $e_{5} \in P$ ) bounds the intersection of $\mathrm{H}_{i}(1 \leqslant i \leqslant 4$ ). The line segment that bounds the intersection is contained by $e_{5}$ and is visible to all the vertices of $P$ 
by definition. For this line segment to degenerate into a single point, $e_{5}$ must contain $v$, which contradicts the definition of $P$.

If $n=5$ and the intersection of $H_{i}(1 \leqslant i \leqslant 4)$ is bounded then $H_{5}$ may or may not change the intersection of $\mathrm{H}_{i}(1 \leqslant i \leqslant 4)$ (refer to Fig. 16.2c). At least one of the line segments that bounds the intersection of $\mathrm{H}_{i}(1 \leqslant i \leqslant 5)$ is contained in the edge of $\mathrm{P}$, otherwise $\mathrm{P}$ has at least three reflex vertices, which is a contradiction. This line segment cannot degenerate into a single point because $\mathrm{P}$ is in general position.

The problem we study is twofold:

1. We are given a simple polygon $P$ of size $n$. Our goal is to position at most $\lfloor 2 n / 3\rfloor$ towers inside $P$ such that every point $p \in P$ can be localized.

2. We want to design a localization algorithm which does not know $P$, but knows that the locations of the towers were computed using the parity trick. For any point $p \in P$, its location can be found by using the coordinates of the towers that see $p$ and the distances from those towers to $p$.

It may seem counter-intuitive, but the knowledge of the parity trick is stronger than the knowledge of the map of P. Some towers (while still on the boundary of some partition) may end up in the interior of $P$. This is not a problem when the parity trick is used but may lead to ambiguities when only the map of $P$ is known (refer for example to Fig. 16.1b).

The following theorem shows that additional knowledge like the parity trick or the map of $\mathrm{P}$ (including its partition) is necessary to achieve localization with the use of less than $n$ towers.

THEOREM 16.3. Let $\mathrm{P}$ be a simple polygon with $\mathrm{n}$ vertices, which is not known to the localization algorithm. Let $\mathrm{n}=3 \mathrm{k}+\mathrm{q}$, for integer $\mathrm{k} \geqslant 1$ and $\mathrm{q}=0,1$ or 2 . Let $\mathrm{T}$ be a set of towers of size less than $\mathrm{n}-\mathrm{q}$. Suppose that the localization algorithm only receives the positions of the towers together with their distances, visible to the agent. Furthermore, assume that the localization algorithm does not know that the towers were placed using the parity trick. Then the agent cannot localize itself.

Proof. Consider the smallest such polygon $P$ with $n=3$. Assume to the contrary, that $P$ can be trilaterated with 2 towers. Given the coordinates of the two towers $t_{1}$ and $t_{2}$ together with the distances to a query point $p$ one can deduce that $p$ is in one of the two possible locations $C\left(t_{1}, d_{1}\right) \cap C\left(t_{2}, d_{2}\right)=$ $\left\{p_{1}, p_{2}\right\}$. But without additional information it is impossible to choose one location over another. Refer to Fig. 16.3. Similarly, any quadrilateral or pentagon requires at least 3 towers to trilaterate it.

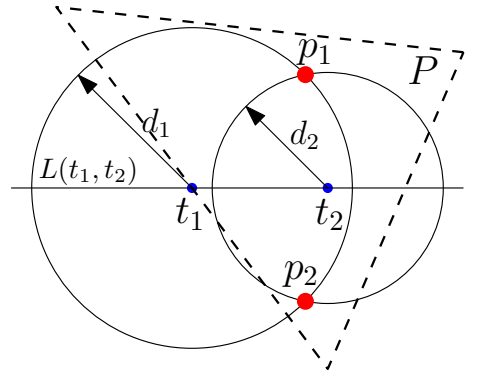

(a)

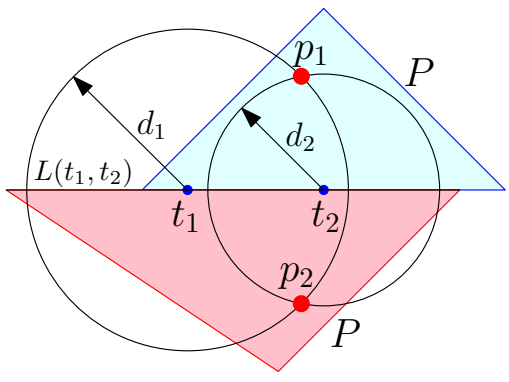

(b)

Figure 16.3: How to locate $p$ if the map of the polygon is unavailable? (a) Ambiguity along $L\left(t_{1}, t_{2}\right)$. We know that $p_{1}$ and/or $p_{2}$ belongs to $P$. (b) We need additional information to tell if $P$ (and $p)$ is above or below $L\left(t_{1}, t_{2}\right)$.

Let $P$ be a comb polygon with $n=3 k+q$ vertices such that one of its $k$ spikes contains $q$ extra vertices. Refer to Fig. 16.4. No point of $P$ can see the complete interior of two different spikes. Assume 
to the contrary that $P$ can be trilaterated with less than $n-q$ towers. In other words, assume that $P$ can be trilaterated with $3 k-1$ towers. It follows that one of the spikes contains less than 3 towers. We showed for smaller polygons (with $n=3,4$ or 5 ) that two towers cannot trilaterate it. Even if we know that the two towers are positioned on the same edge of $\mathrm{P}$, it is unknown to which side of the edge the interior of the polygon resides, and thus the localization is not possible. Observe that no polygon or spike can be trilaterated with one or no towers.

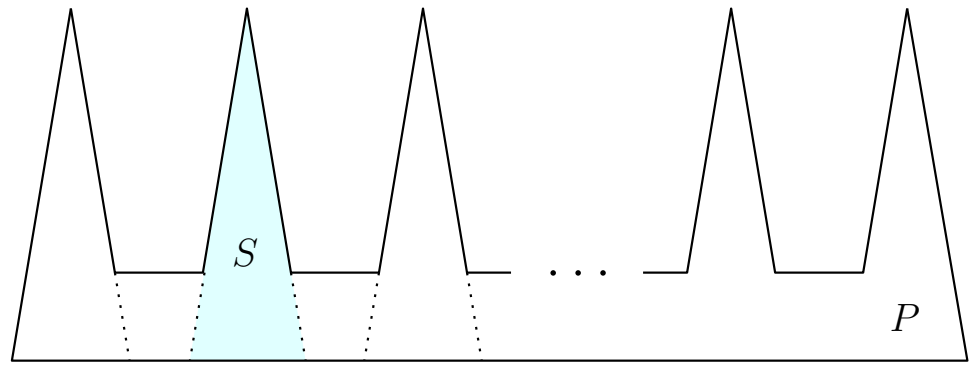

(a)

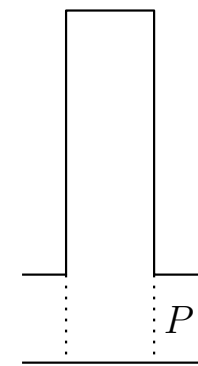

(b)

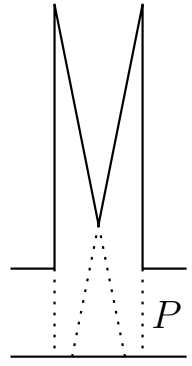

(c)

Figure 16.4: Comb polygon with $n=3 k+q$ vertices. (a) One of the $k$ spikes $S$ is shown in cyan. (b) One of the spikes when $q=1$. (c) One of the spikes when $q=2$. 


\section{TÓTH'S PARTITION}

\section{7}

A simple polygonal chain $\mathrm{c}$ is a cut of a polygon $\mathrm{P}$ if the two endpoints of $\mathrm{c}$ belong to $\partial \mathrm{P}$ and all interior points of $c$ are interior points of $P$. A cut $c$ decomposes $P$ into two polygons $P_{1}$ and $P_{2}$ such that $P=P_{1} \cup P_{2}$ and $c=P_{1} \cap P_{2}$. A diagonal of $P$ is a line segment that connects non-adjacent vertices of $\mathrm{P}$ and is contained in or on the boundary of $\mathrm{P}$. If $\mathrm{c}$ is a cut and a diagonal of $\mathrm{P}$ then it is called a diagonal cut.

Tóth showed in [44] that any simple polygon in general position of size $n$ can be guarded by $\lfloor n / 3\rfloor$ point guards whose range of vision is $180^{\circ}$ (let us call this result Tóth's Theorem). His approach is to decompose $\mathrm{P}$ into subpolygons via cuts and to specify the locations of the guards. The cuts are composed of one or two line segments and are not restricted to be diagonal cuts. He uses a constructive induction to show his main result. Let $n_{1}$ (respectively $n_{2}$ ) denote the size of $P_{1}$ (respectively $P_{2}$ ). When P contains a cut that satisfies $\left\lfloor\frac{n_{1}}{3}\right\rfloor+\left\lfloor\frac{n_{2}}{3}\right\rfloor \leqslant\left\lfloor\frac{n}{3}\right\rfloor$, the proof of Tóth's Theorem can be completed by applying induction to both $\mathrm{P}_{1}$ and $\mathrm{P}_{2}$.

Tóth's method heavily relies upon the partitioning of a polygon into subpolygons (on which he can apply induction). He performs diagonal cuts whenever it is possible, otherwise he cuts along a continuation of some edge of P; along a two-line segment made of an extension of two edges of $\mathrm{P}$ that intersect inside P; or along the bisector of a reflex vertex of P. Notice that the three latter types of cuts may introduce new vertices that are not necessarily in general position with the given set of vertices.

However, Tóth assumes that every cut produces polygons in general position, which is a very strong assumption. We strengthen the work [44] by lifting this assumption and reproving Tóth's result. We assume that the input polygon is in general position, while non-general position may occur for subpolygons of the partition. Moreover, we found and fixed several mistakes in [44].

DEFINITION 17.1 (Good Cut). Let $\mathrm{n}>5$. A cut is called a good cut if it satisfies the following: $\left\lfloor\frac{\mathrm{n}_{1}}{3}\right\rfloor+$ $\left\lfloor\frac{\mathfrak{n}_{2}}{3}\right\rfloor \leqslant\left\lfloor\frac{\mathfrak{n}}{3}\right\rfloor$. If a good cut is a diagonal then it is called a good diagonal cut.

To avoid confusion, let $\mathrm{P}^{\prime}$ refer to a polygon to which we apply the inductive partitioning. In other words, $\mathrm{P}^{\prime}$ can refer to the input polygon $\mathrm{P}$ before any partition was applied as well as to any subpolygon of $\mathrm{P}$ obtained during the partitioning of $\mathrm{P}$. Recall that $\mathrm{P}$ is in general position, while $\mathrm{P}^{\prime}$ may not be in general position.

If the polygon is not in general position then its triangulation may include triangles whose three vertices are collinear. We call such triangles degenerate triangles. Refer to Fig. 17.1, showing an example of a degenerate triangle $\Delta v_{1} v_{2} v_{3}$. The diagonal $\overline{v_{1} v_{3}}$ cannot be a good diagonal cut even if it partitions $\mathrm{P}^{\prime}$ into $\mathrm{P}_{1}$ and $\mathrm{P}_{2}$ such that $\left\lfloor\frac{\mathrm{n}_{1}}{3}\right\rfloor+\left\lfloor\frac{\mathrm{n}_{2}}{3}\right\rfloor \leqslant\left\lfloor\frac{n}{3}\right\rfloor$, because interior points of a cut cannot contain points of $\partial \mathrm{P}^{\prime}$.

To extend Tóth's partition to polygons in non-general position we need to extend the definition of a cut. A simple polygonal chain $\mathrm{c}^{\prime}$ is a dissection of $\mathrm{P}$ if the two endpoints of $\mathrm{c}^{\prime}$ belong to $\partial \mathrm{P}$ and all interior points of $c^{\prime}$ are either interior points of $\mathrm{P}$ or belong to $\partial \mathrm{P}$. A dissection $\mathrm{c}^{\prime}$ decomposes $\mathrm{P}$ into two polygons $P_{1}$ and $P_{2}$ (that are not necessarily in general position) such that $P=P_{1} \cup P_{2}$ and $c^{\prime}=\mathrm{P}_{1} \cap \mathrm{P}_{2}$. If $\mathrm{c}^{\prime}$ is a dissection and a diagonal of $\mathrm{P}$ then it is called a diagonal dissection.

DEFINITION 17.2 (Good Dissection). Let $\mathrm{n}>5$. A dissection is called a good dissection if $\left\lfloor\frac{n_{1}}{3}\right\rfloor+\left\lfloor\frac{n_{2}}{3}\right\rfloor \leqslant$ $\left\lfloor\frac{\mathfrak{n}}{3}\right\rfloor$.

We extend the results in [44] by removing the assumption that the partitioning produces subpolygons in general position and by thus strengthening the result. In this thesis, we need to refer to many 
Lemmas, Propositions and Claims from [44]. Indeed, we apply some of these results, we fill the gaps in some proofs of these results and we generalize some others. In order for this part of the thesis to be self-contained, we write the statements of all these results with their original numbering [44].

Simplification Step: If $\mathrm{P}^{\prime}$ has consecutive vertices $v_{1}, v_{2}$ and $v_{3}$ along $\partial \mathrm{P}^{\prime}$ that are collinear then:

1. If the angle of $\mathrm{P}^{\prime}$ at $v_{2}$ is $\pi$ then replace $v_{2}$ together with its adjacent edges by the edge $\overline{v_{1} v_{3}}$.

2. If the angle of $\mathrm{P}^{\prime}$ at $v_{2}$ is 0 or $2 \pi$ then delete $v_{2}$ together with its adjacent edges and add the edge $\overline{v_{1} v_{3}}$. Assume w.l.o.g. that the distance between $v_{3}$ and $v_{2}$ is smaller than the distance between $v_{1}$ and $v_{2}$. The line segment $\overline{v_{2} v_{3}}$ will be guarded despite that it was removed from $\mathrm{P}^{\prime}$. Refer to the following subsections for examples.

In both cases we denote the updated polygon by $\mathrm{P}^{\prime}$; its number of vertices decreased by 1 .

Assume for simplicity that $P^{\prime}$ does not contain consecutive collinear vertices along $\partial P^{\prime}$. Let $n>5$ be the number of vertices of $P^{\prime}$. Let $k$ be a positive integer and $q \in\{0,1,2\}$ such that $n=3 k+q$. Notice that a diagonal dissects $P^{\prime}$ into $P_{1}$ (of size $n_{1}$ ) and $P_{2}$ (of size $n_{2}$ ) such that $n_{1}+n_{2}=n+2$.

The proofs of the following three propositions are identical to those in Tóth's paper.

то́тн'S PROPOSITION 1. For $\mathrm{P}^{\prime}$ with $\mathrm{n}=3 \mathrm{k}$ any diagonal is a good dissection.

TÓтH'S PROPOSITION 2. For $\mathrm{P}^{\prime}$ with $\mathrm{n}=3 \mathrm{k}+1$ there exists a diagonal that represents a good dissection.

Tóth's proposition 3. For $\mathrm{P}^{\prime}$ with $\mathrm{n}=3 \mathrm{k}+2$ a dissection is a good dissection if it decomposes $\mathrm{P}^{\prime}$ into $\mathrm{P}_{1}$ and $\mathrm{P}_{2}$ (not necessarily simple polygons) such that $\mathrm{n}_{1}=3 \mathrm{k}_{1}+2$ and $\mathrm{n}_{2}=3 \mathrm{k}_{2}+2$ (for $\mathrm{k}_{1}+\mathrm{k}_{2}=\mathrm{k}$ ).

17.1 CASE STUDY

In this section we study how Propositions 1,2 and 3 can be applied to polygons in non-general position. Let $\Delta v_{1} v_{2} v_{3}$ be a minimum degenerate triangle in $\mathrm{P}^{\prime}$, i.e. it does not contain any vertex of $\mathrm{P}^{\prime}$ other than $v_{1}, v_{2}$ or $v_{3}$. Consider the example depicted in Fig. 17.1. The diagonal $\overline{v_{1} v_{3}}$ partitions $\mathrm{P}^{\prime}$ into two polygons: $\mathrm{P}_{1}$ and $\mathrm{P}_{2}\left(\mathrm{P}_{2}\right.$ can be further viewed as a union of two subpolygons $\mathrm{P}_{\mathrm{a}}$ and $\mathrm{P}_{\mathrm{b}}$ ). Notice that $v_{2} \notin \mathrm{P}_{1}$. There is a possibility for $\mathrm{P}^{\prime}$ to have a vertex $v^{\prime} \notin\left\{v_{1}, v_{2}, v_{3}\right\}$ such that $v^{\prime} \in \mathrm{L}_{v_{1} v_{3}}$. However $v^{\prime}$ cannot belong to the line segment $\overline{v_{1} v_{3}}$, otherwise, the triangle $\Delta v_{1} v_{2} v_{3}$ is not minimum, and we can choose $\Delta v_{1} v_{2} v^{\prime}$ or $\Delta v^{\prime} v_{2} v_{3}$ instead. It follows that no vertex of $\mathrm{P}_{1}$ (respectively $\mathrm{P}_{\mathrm{a}}, \mathrm{P}_{\mathrm{b}}$ ) is located on the edge $\overline{v_{1} v_{3}}$ (respectively $\overline{v_{1} v_{2}}$, $\left.\overline{v_{2} v_{3}}\right)$. Notice that $\mathrm{P}^{\prime}$ may contain an edge $e^{\prime}$ that contains

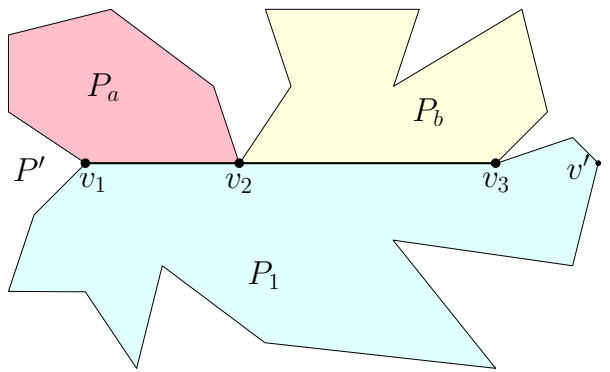

Figure 17.1: The diagonal $\overline{v_{1} v_{3}}$ contains a vertex $v_{2}$ of $\mathrm{P}^{\prime} . \mathrm{P}_{2}=\mathrm{P}_{\mathrm{a}} \cup$ $\mathrm{P}_{\mathrm{b}}$. Notice that $v_{2} \notin \mathrm{P}_{1}$. $\overline{v_{1} v_{3}}$; $P_{1}$ inherits it (since $v_{2} \notin P_{1}$ ), but it won't cause any trouble because of the "simplification" step described in the beginning of this chapter. Notice also, that two vertices of $\mathrm{P}^{\prime}$ cannot be at the same location.

Assume that $\overline{v_{1} v_{3}}$ is a good dissection, i.e. it decomposes $P^{\prime}$ into $P_{1}$ and $P_{2}$ such that $\left\lfloor\frac{n_{1}}{3}\right\rfloor+\left\lfloor\frac{n_{2}}{3}\right\rfloor \leqslant$ $\left\lfloor\frac{n}{3}\right\rfloor$. Assume also that $n>5$. Let $n_{a}$ (respectively $n_{b}$ ) be the number of vertices of $P_{a}$ (respectively $\left.P_{b}\right)$. Notice that $n_{a}+n_{b}=n_{2}+1$ because $v_{2}$ was counted twice. When it is possible, we prefer to avoid cutting along the diagonal $\overline{v_{1} v_{3}}$. However, when necessary, it can be done in the following way. We consider three cases (refer to Fig. 17.1):

Case 1: $n=3 k$. By Tóth's Proposition I every diagonal is a good dissection. If $\mathrm{P}_{\mathrm{a}}$ is not composed of the line segment $\overline{v_{1} v_{2}}$ only, then $\overline{v_{1} v_{2}}$ is a good diagonal dissection, that partitions $\mathrm{P}^{\prime}$ into two polygons 
$\mathrm{P}_{\mathrm{a}}$ and $\mathrm{P}_{1} \cup \mathrm{P}_{\mathrm{b}}$. Otherwise, $\overline{v_{2} v_{3}}$ is a good diagonal dissection, that partitions $\mathrm{P}^{\prime}$ into two polygons $\mathrm{P}_{\mathrm{b}}$ and $\mathrm{P}_{1} \cup \mathrm{P}_{\mathrm{a}}$. Notice that $\overline{v_{1} v_{2}}$ and $\overline{v_{2} v_{3}}$ cannot be both edges of $\mathrm{P}^{\prime}$ because of the simplification step we applied to $\mathrm{P}^{\prime}$.

Alternatively, we can dissect $\mathrm{P}^{\prime}$ along $\overline{v_{1} \nu_{3}}$. The polygon $\mathrm{P}_{2}$ has an edge $\overline{v_{1} v_{3}}$ that contains vertex $v_{2}$. If a $180^{\circ}$-guard is located at $v_{2}$ for any subpolygon $\mathrm{P}_{2}^{\prime}$ of $\mathrm{P}_{2}$, then we position the towers close to $v_{2}$ on a line segment that contains $v_{2}$ (but not necessarily in kernel $\left(\mathrm{P}_{2}^{\prime}\right) \cap \partial \mathrm{P}_{2}^{\prime}$ ) only if $v_{2}$ is not a vertex of the original polygon P. However, if $v_{2} \in \mathrm{P}$ then we position our towers in the close proximity to $v_{2}$ but in the interior of $P_{1}$, oriented towards $P_{2}$.

Case 2: $n=3 k+1$. Since $\overline{v_{1} v_{3}}$ decomposes $P^{\prime}$ into $P_{1}$ and $P_{2}$ such that $\left\lfloor\frac{n_{1}}{3}\right\rfloor+\left\lfloor\frac{n_{2}}{3}\right\rfloor \leqslant\left\lfloor\frac{n}{3}\right\rfloor$ then either $n_{1}=3 k_{1}+1$ and $n_{2}=3 k_{2}+2$, or $n_{1}=3 k_{1}+2$ and $n_{2}=3 k_{2}+1$ for $k_{1}+k_{2}=k$. If $\overline{v_{1} v_{2}}$ is an edge of $\mathrm{P}^{\prime}$ (meaning that $\mathrm{P}_{\mathrm{a}}$ consists of a line segment $\overline{v_{1} v_{2}}$ only) then we can dissect $\mathrm{P}^{\prime}$ either along $\overline{v_{2} v_{3}}$ or along $\overline{v_{1} v_{3}}$. In the former case $\mathrm{P}^{\prime}$ will be decomposed into $\mathrm{P}_{1} \cup\left\{v_{2}\right\}$ and $\mathrm{P}_{\mathrm{b}}\left(v_{2}\right.$ will be deleted from $P_{1} \cup\left\{v_{2}\right\}$ during the simplification step to obtain $\left.P_{1}\right)$. In the latter case $P^{\prime}$ will be decomposed into $P_{1}$ and $P_{b} \cup\left\{v_{1}\right\}\left(v_{1}\right.$ will be removed from $P_{b} \cup\left\{v_{1}\right\}$ during the simplification step to obtain $P_{b}$ ). In either case we do not have to specifically guard the line segment $\overline{v_{1} v_{2}}$. If $\mathrm{P}_{1}$ is guarded then so is $\overline{v_{1} v_{2}}$. Similarly treat the case where $\overline{v_{2} v_{3}}$ is an edge of $\mathrm{P}^{\prime}$.

Assume that $n_{a}, n_{b}>2$. Recall, that $n_{b}=n_{2}-n_{a}+1$. Several cases are possible:

- $\mathrm{n}_{\mathrm{a}}=3 \mathrm{k}_{\mathrm{a}}+1$. Then we dissect $\mathrm{P}^{\prime}$ along $\overline{v_{1} v_{2}}$. This partition creates the polygons $\mathrm{P}_{\mathrm{a}}$ of size $n_{a}=3 k_{a}+1$ and $P_{1} \cup P_{b}$ of size $3\left(k_{1}+k_{2}-k_{a}\right)+2$.

- $\mathrm{n}_{\mathrm{a}}=3 \mathrm{k}_{\mathrm{a}}+2$. Then we dissect $\mathrm{P}^{\prime}$ along $\overline{v_{1} v_{2}}$. This partition creates the polygons $\mathrm{P}_{\mathrm{a}}$ of size $n_{a}=3 k_{a}+2$ and $P_{1} \cup P_{b}$ of size $3\left(k_{1}+k_{2}-k_{a}\right)+1$.

$>\mathrm{n}_{\mathrm{a}}=3 \mathrm{k}_{\mathrm{a}}$. Then:

$-n_{1}=3 k_{1}+2$ and $n_{2}=3 k_{2}+1$. Then we dissect $P^{\prime}$ along $\overline{v_{2} v_{3}}$. This partition creates the polygons $P_{b}$ of size $n_{b}=3\left(k_{2}-k_{a}\right)+2$ and $P_{1} \cup P_{a}$ of size $3\left(k_{1}+k_{a}\right)+1$.

$-n_{1}=3 k_{1}+1$ and $n_{2}=3 k_{2}+2$. Then both $\overline{v_{1} v_{2}}$ and $\overline{v_{2} v_{3}}$ are not good diagonal cuts. We cannot avoid dissecting along $\overline{v_{1} v_{3}}$. If a $180^{\circ}$-guard eventually needs to be positioned at $v_{2}$ (for any subpolygon of $\mathrm{P}_{2}$ ) and $v_{2}$ is a vertex of $\mathrm{P}$, then we position our towers close to $v_{2}$ but in the interior of $\mathrm{P}_{1}$, oriented towards $\mathrm{P}_{2}$.

Case $3: n=3 k+2$. In this case $n_{1}=3 k_{1}+2$ and $n_{2}=3 k_{2}+2$. If $n_{a}=2$ then either dissect $P^{\prime}$ along $\overline{v_{2} v_{3}}$ (and later delete $v_{2}$ from $P_{1} \cup v_{2}$ during the simplification step), or dissect $P^{\prime}$ along $\overline{v_{1} v_{3}}$ (delete $v_{1}$ from $\mathrm{P}_{\mathrm{b}} \cup v_{1}$ during the simplification step). Notice that in both cases $\overline{v_{1} v_{2}}$ will be guarded. The case where $n_{b}=2$ is similar.

If $n_{a}, n_{b}>2$ then we have no choice but to dissect along $\overline{v_{1} v_{3}}$. This creates a polygon $P_{2}$ whose kernel degenerates into a single point $v_{2}$. It is not a problem for $180^{\circ}$-guards but it is a serious obstacle for our problem. If $v_{2}$ is a vertex of $\mathrm{P}$ and a $180^{\circ}$-guard is located at $v_{2}$ for any subpolygon of $\mathrm{P}_{2}$ then we position our towers close to $v_{2}$ but in the interior of $P_{1}$, and orient those towers towards $P_{2}$. We consider the general approach to this problem in the following section.

\subsection{POINT-KERNEL PROBLEM}

In Section 17.1 we studied simple cases where a diagonal dissection is applied to polygons in nongeneral position. In this section, we show how to circumvent some difficulties that arise when adapting Tóth's partitioning to our problem.

The dissection of $\mathrm{P}$ may create subpolygons that are not in general position. This means that the partition of $\mathrm{P}$ may contain star-shaped polygons whose kernels degenerate into a single point. While this is not a problem for $180^{\circ}$-guards, it is a serious obstacle for tower positioning. Indeed, we need at least two distinct points in the kernel of each part of the partition to trilaterate $\mathrm{P}$. 
Let $\overline{v_{1} v_{4}}$ be a good dissection, i.e. it decomposes $P^{\prime}$ into $P_{1}$ and $P_{2}$ such that $\left\lfloor\frac{n_{1}}{3}\right\rfloor+\left\lfloor\frac{n_{2}}{3}\right\rfloor \leqslant$ $\left\lfloor\frac{n}{3}\right\rfloor$. Assume also that $n>5$. Assume that $\overline{v_{1} v_{4}}$ contains at least 2 more vertices of $\mathrm{P}^{\prime}$ in its interior. Let $v_{2}, v_{3} \in \overline{v_{1} v_{4}}$. Refer to Fig. 17.2. Notice that $v_{2}$ and $v_{3}$ belong to subpolygons of $\mathrm{P}^{\prime}$ on the opposite sides of $\mathrm{L}\left(v_{1}, v_{4}\right)$. Recall that the original polygon $\mathrm{P}$ is given in general position. Thus at most two vertices of $P$ belong to the same line. If $v_{2}$ and $v_{3}$ are not both vertices of $P$ then there is no problem for tower positioning, and the dissection via $\overline{v_{1} v_{4}}$ can be applied directly. We then follow Tóth's partition and replace every $180^{\circ}$ -

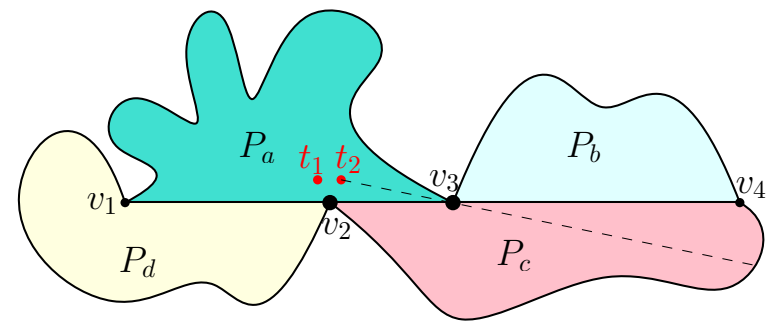

Figure 17.2: $\overline{v_{1} v_{4}}$ is a good diagonal dissection that contains exactly two vertices of $P: v_{2}$ and $v_{3} \cdot \mathrm{P}_{1}=\mathrm{P}_{\mathrm{a}} \cup \mathrm{P}_{\mathrm{b}}$ and $\mathrm{P}_{2}=\mathrm{P}_{\mathrm{c}} \cup \mathrm{P}_{\mathrm{d}}$. Notice that $v_{2} \notin \mathrm{P}_{\mathrm{a}}$ and $\nu_{3} \notin \mathrm{P}_{\mathrm{c}}$. guard with a pair of towers in the guard's vicinity. However, if $v_{2}$ and $v_{3}$ are vertices of $\mathrm{P}$ then the visibility of towers can be obstructed. Consider the example of Fig. 17.2. Assume that a $180^{\circ}$-guard is positioned at $v_{2}$ to observe a subpolygon of $\mathrm{P}_{\mathrm{c}} \cup \mathrm{P}_{\mathrm{d}}$ (say, pentagon $v_{1} v_{4} v_{\mathrm{c}} v_{2} v_{\mathrm{d}}$ for $v_{\mathrm{c}} \in \mathrm{P}_{\mathrm{c}}$ and $v_{\mathrm{d}} \in \mathrm{P}_{\mathrm{d}}$ ). We cannot replace the $180^{\circ}$-guard with a pair of towers because the kernel of the pentagon degenerates into a single point $v_{2}$. Our attempt to position towers in the vicinity of $v_{2}$ but interior to $P_{a}$ is not successful either, because $v_{3}$, as a vertex of $P$, blocks visibility with respect to $P_{c}$. We have to find another dissection. In general, we are looking for a dissection that destroys the diagonal $\overline{v_{1} v_{4}}$.

Assume that $v_{2}$ and $v_{3}$ are vertices of $\mathrm{P}$. It follows that there are no vertices of $\mathrm{P}$ on $\mathrm{L}\left(v_{1}, v_{4}\right)$ other than $v_{2}$ and $v_{3}$. Assume that $\overline{v_{1} v_{4}}$ is a good dissection. If one of $\overline{v_{1} v_{3}}, \overline{v_{1} v_{2}}, \overline{v_{3} v_{4}}, \overline{v_{2} v_{4}}$ or $\overline{v_{2} v_{3}}$ is a good dissection then we cut along it. So assume that none of those diagonals represent a good dissection. It follows from Tóth's Proposition 1 that $n \neq 3 k$. Consider the following cases:

Case 1: $n=3 k+1$. By Tóth's Proposition 2 either $n_{1}=3 k_{1}+2$ and $n_{2}=3 k_{2}+1$, or $n_{1}=3 k_{1}+1$ and $n_{2}=3 k_{2}+2$ for $k_{1}+k_{2}=k$, where $P_{1}=P_{a} \cup P_{b}$ and $P_{2}=P_{c} \cup P_{d}$. Assume w.l.o.g. that $n_{1}=3 k_{1}+2$ and $n_{2}=3 k_{2}+1$. We assumed that $\overline{v_{2} v_{3}}$ is not a good dissection and thus the size of $P_{c} \cup P_{b}$ is a multiple of 3 and the size of $P_{d} \cup P_{a}$ is a multiple of 3. Consider the following three subcases:

Case 1.1: $\left|P_{d}\right|=3 k_{d}$; thus $\left|P_{c}\right|=3 k_{c}+2$ or $\left|P_{c}\right|=2$. Since the size of $P_{c} \cup P_{b}$ is a multiple of 3, we have $\left|\mathrm{P}_{\mathrm{b}}\right|=3 \mathrm{k}_{\mathrm{b}}+2$ or $\left|\mathrm{P}_{\mathrm{b}}\right|=2$. Notice that $\mathrm{P}_{\mathrm{b}}$ and $\mathrm{P}_{\mathrm{c}}$ cannot be both of size 2 , otherwise $v_{4}$ would be deleted during the simplification step. Both diagonals $\overline{v_{3} v_{4}}$ and $\overline{v_{2} v_{4}}$ represent a good dissection. Thus, if $\left|\mathrm{P}_{\mathrm{c}}\right| \neq 2$ then dissect along $\overline{v_{2} v_{4}}$; if $\left|\mathrm{P}_{\mathrm{b}}\right| \neq 2$ then dissect along $\overline{v_{3} v_{4}}$. The polygon $\mathrm{P}_{\mathrm{a}} \cup \mathrm{P}_{\mathrm{d}} \cup \triangle v_{2} v_{3} v_{4}$ will be simplified and it will loose $\overline{v_{3} v_{4}}$ (which is guarded by $\mathrm{P}_{\mathrm{b}}$ or/and $\mathrm{P}_{\mathrm{c}}$ ).

Case 1.2: $\left|P_{d}\right|=3 k_{d}+1$ or $\left|P_{d}\right|=3 k_{d}+2$. Then $\overline{v_{1} v_{2}}$ is a good diagonal dissection.

Case 1.3: $\left|P_{d}\right|=2$. Then $\left|P_{a}\right|=3 k_{a}+2$ and thus $\overline{v_{1} v_{3}}$ is a good diagonal dissection, which creates the polygons $\mathrm{P}_{\mathrm{a}}$ and $\mathrm{P}_{\mathrm{b}} \cup \mathrm{P}_{\mathrm{c}} \cup\left\{v_{1}\right\}$. The vertex $v_{1}$ will be deleted from $\mathrm{P}_{\mathrm{b}} \cup \mathrm{P}_{\mathrm{c}} \cup\left\{v_{1}\right\}$ during the simplification step. Notice that the line segment $\overline{\nu_{1} v_{2}}$ will be guarded by $P_{a}$.

Case 2: $\mathrm{n}=3 \mathrm{k}+2$. Since $\overline{v_{1} v_{4}}$ is a good dissection, it partitions $\mathrm{P}^{\prime}$ into $\mathrm{P}_{1}=\mathrm{P}_{\mathrm{a}} \cup \mathrm{P}_{\mathrm{b}}$ of size $n_{1}=3 k_{1}+2$ and $P_{2}=P_{c} \cup P_{d}$ of size $n_{2}=3 k_{2}+2$.

Case 2.1: $\left|\mathrm{P}_{\mathrm{b}}\right|=3 \mathrm{k}_{\mathrm{b}}+1$; thus $\left|\mathrm{P}_{\mathrm{a}}\right|=3 \mathrm{k}_{\mathrm{a}}+2$ or $\left|\mathrm{P}_{\mathrm{a}}\right|=2$. We assumed that $\overline{v_{1} v_{3}}$ is not a good dissection and thus $\left|P_{a}\right| \neq 3 k_{a}+2$. It follows that $P_{a}$ is a line segment $\overline{v_{1} v_{3}}$. Symmetrically, if $\left|P_{d}\right|=$ $3 k_{d}+1$ then $\left|P_{c}\right|=2$. If $\left|P_{b}\right|=3 k_{b}+1$ and $\left|P_{d}\right|=3 k_{d}+1$ then we have a good dissection $\overline{v_{2} v_{3}}$, which is a contradiction.

The case where $\left|P_{b}\right|=3 k_{b}+1$ and $\left|P_{c}\right|=3 k_{c}+1$ is not possible because in this case $P_{a}=\overline{v_{1} v_{3}}$ and $\mathrm{P}_{\mathrm{d}}=\overline{v_{1} v_{2}}$ and thus $v_{1}$ would not survive the simplification step.

Thus, if $\left|\mathrm{P}_{\mathrm{b}}\right|=3 \mathrm{k}_{\mathrm{b}}+1$ then $\left|\mathrm{P}_{\mathrm{c}}\right|=3 \mathrm{k}_{\mathrm{c}}$ and $\left|\mathrm{P}_{\mathrm{d}}\right|=3 \mathrm{k}_{\mathrm{d}}$. Refer to Fig. 17.3. Let $v_{2}^{\prime}$ be an immediate neighbour of $v_{2}$ such that $v_{2}^{\prime}$ is a vertex of $\mathrm{P}_{\mathrm{c}}$ and $v_{2}^{\prime} \neq v_{4}$. If $v_{2}^{\prime}$ and $v_{3}$ see each other, then $\overline{v_{2}^{\prime} v_{3}}$ 
is a diagonal of $\mathrm{P}^{\prime}$. It dissects $\mathrm{P}^{\prime}$ into two subpolygons $\mathrm{P}_{\mathrm{d}} \cup \triangle v_{2} v_{3} v_{2}^{\prime}$ of size $3 \mathrm{k}_{\mathrm{d}}+2$ and $\left(\mathrm{P}_{\mathrm{b}} \cup \mathrm{P}_{\mathrm{c}}\right) \backslash$ $\triangle v_{2} v_{3} v_{2}^{\prime}$ of size $3 k_{b}+3 k_{c}-1=3\left(k_{b}+k_{c}-1\right)+2$.

Assume now that $v_{2}^{\prime}$ and $v_{3}$ do not see each other. Let us give numerical labels to the vertices of $P_{c}$ as follows: $v_{2}$ gets label $1, v_{2}^{\prime}$ gets 2 , and so on, $v_{4}$ will be labelled $3 k_{c}$. If $v_{3}$ can see a vertex of $P_{c}$ with label whose value modulo 3 equals 2 then dissect $P^{\prime}$ along the diagonal that connects $v_{3}$ and that vertex. If no such vertex can be seen from $v_{3}$, we do the following. Let $v_{3}^{\prime}$ be an immediate neighbour of $v_{3}$ such that $v_{3}^{\prime}$ is a vertex of $\mathrm{P}_{\mathrm{b}}$ and $v_{3}^{\prime} \neq v_{4}$. Let $v_{3}^{\prime \prime}$ be the point on $\partial \mathrm{P}_{\mathrm{c}}$ where the line

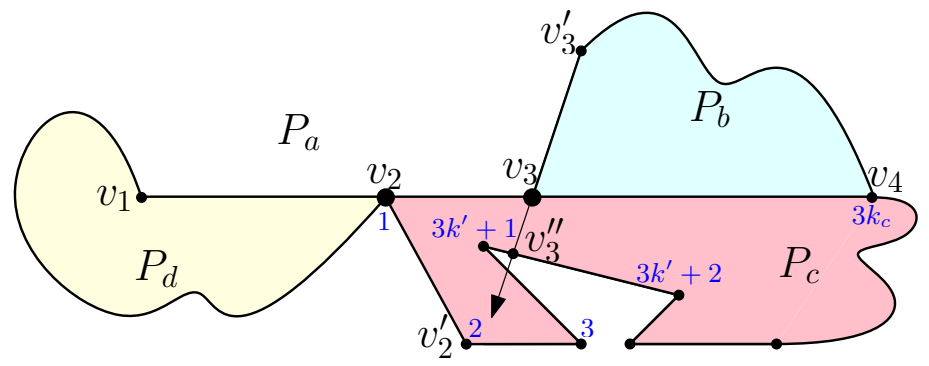

Figure 17.3: $\overline{v_{1} v_{4}}$ is a good diagonal dissection that contains exactly two vertices of $P: v_{2}$ and $v_{3} . P_{a}=\left\{v_{1}, v_{3}\right\}$, $\left|\mathrm{P}_{\mathrm{b}}\right|=3 \mathrm{k}_{\mathrm{b}}+1,\left|\mathrm{P}_{\mathrm{c}}\right|=3 \mathrm{k}_{\mathrm{c}}$ and $\left|\mathrm{P}_{\mathrm{d}}\right|=3 \mathrm{k}_{\mathrm{d}}$. supporting $\overline{v_{3}^{\prime} v_{3}}$ first hits $\partial \mathrm{P}_{\mathrm{c}}$ (refer to Fig. 17.3). If $v_{3}^{\prime \prime}$ belongs to an edge with vertices whose labels modulo 3 equal 1 and 2 then dissect $\mathrm{P}^{\prime}$

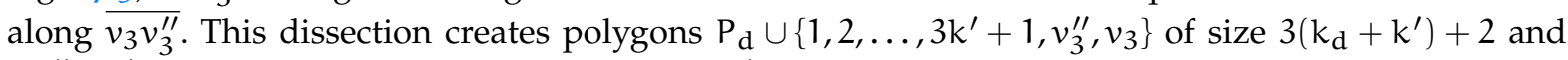
$\left(\left\{v_{3}^{\prime \prime}, 3 k^{\prime}+2, \ldots 3 k_{c}\right\} \cup P_{b}\right) \backslash\left\{v_{3}\right\}$ of size $3\left(k_{c}-k^{\prime}+k_{b}-1\right)+2$.

If this scenario have not worked, we consider the worst case possible: We assume that $\mathrm{P}_{2}=\mathrm{P}_{\mathrm{c}} \cup \mathrm{P}_{\mathrm{d}}$ has undergone some partitioning that resulted in the creation of the pentagon $v_{1}^{\prime} v_{4}^{\prime} v_{\mathrm{c}} v_{2} v_{\mathrm{d}}$ for $v_{\mathrm{c}}, v_{4}^{\prime} \in$ $\mathrm{P}_{\mathrm{c}}, v_{\mathrm{d}}, v_{1}^{\prime} \in \mathrm{P}_{\mathrm{d}}$ such that $v_{1}^{\prime}, v_{4}^{\prime} \in \mathrm{L}\left(v_{2}, v_{3}\right)$ and $v_{4}^{\prime} \in \overline{v_{3} v_{4}}$ (refer to Fig. 17.4). Notice that if $v_{4}^{\prime} \in \overline{v_{2} v_{3}}$ or $v_{4}^{\prime}=v_{3}$ then a pair of towers in the vicinity of $v_{2}$ can oversee the pentagon, because $v_{3}$ is not causing an obstruction in this case. Despite that $P_{a}$ is a line segment, the original polygon $\mathrm{P}$ has a nonempty interior in the vicinity of $v_{2}$ to the right of the ray $\overrightarrow{v_{3} v_{2}}$. Notice that $v_{1}^{\prime}$ may be equal to $v_{1}$; $v_{4}^{\prime}$ may be equal to $v_{4}$. Moreover, $v_{\mathrm{c}}$ and $v_{4}^{\prime}$ may not be vertices of $P_{1}$ but were created during the partition. We also assume that $\mathrm{P}_{1}=\mathrm{P}_{\mathrm{b}} \cup\left\{v_{1}\right\}$ was not partitioned yet. Let $P_{c}^{\prime}$ be a subpolygon of $P_{c}$ that inherits the partition of $P_{c}$ to the side of the dissection $\overline{v_{c} v_{4}^{\prime}}$ that contains $v_{4}$ (refer Fig. 17.5). Notice that $v_{c}$ and $v_{3}$ can see each other. If $\overline{v_{c} v_{4}^{\prime}}$ is a diagonal dissection then instead use $\overline{v_{c} v_{3}}$. In this case $v_{4}^{\prime}$ can be deleted and $v_{3}$ is added to $\mathrm{P}_{\mathrm{c}}^{\prime}$.

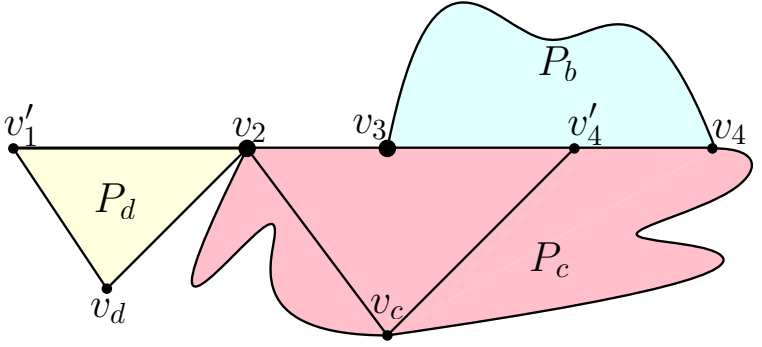

Figure 17.4: $v_{2}$ and $v_{3}$ are vertices of $\mathrm{P}$. It is impossible to localize an agent in the pentagon $v_{1}^{\prime} v_{4}^{\prime} v_{\mathrm{c}} v_{2} v_{\mathrm{d}}$ with a pair of towers only. $\left|\mathrm{P}_{\mathrm{b}}\right|=3 \mathrm{k}_{\mathrm{b}}+1,\left|\mathrm{P}_{\mathrm{c}}\right|=3 \mathrm{k}_{\mathrm{c}}$ and $\left|\mathrm{P}_{\mathrm{d}}\right|=3 \mathrm{k}_{\mathrm{d}}$. The size of $\mathrm{P}_{\mathrm{c}}^{\prime}$ does not change and the number of guards required to guard $\mathrm{P}_{\mathrm{c}}^{\prime}$ does not increase. A pair of towers in the vicinity of $v_{2}$ can oversee the pentagon $v_{1}^{\prime} v_{3} v_{\mathrm{c}} v_{2} v_{\mathrm{d}}$.

If the dissection $\overline{v_{c} v_{4}^{\prime}}$ is a continuation of an edge of $\mathrm{P}_{\mathrm{c}}$ (let $v$ be a vertex that is adjacent to this edge) then two cases are possible. In the first case, the edge $\overline{v_{\mathrm{c}} v}$ that produced the dissection is contained in $\overline{v_{c} v_{4}^{\prime}}$ (refer Fig. 17.5a). In this case, instead of dissecting along $\overline{v_{c} v_{4}^{\prime}}$ use $\overline{v v_{3}}$. The size of $\mathrm{P}_{\mathrm{c}}^{\prime}$ does not change and the hexagon $v_{1}^{\prime} v_{3} \nu v_{c} v_{2} v_{\mathrm{d}}$ can be guarded by a pair of towers in the vicinity of $v_{2}$ (because $v_{2} v_{\mathrm{c}} v v_{3}$ is convex). In the second case the edge $\overline{v_{\mathrm{c}} v}$ that produced the dissection $\overline{v_{\mathrm{c}} v_{4}^{\prime}}$ is not contained in $\overline{v_{c} v_{4}^{\prime}}$ (refer Fig. 17.5b). In the worst case the size of $P_{c}^{\prime}$ is $3 k_{c^{\prime}}+2$. If instead of dissecting along $\overline{v_{c} v_{4}^{\prime}}$ we use $\overline{v_{c} v_{3}}$ then the size of $P_{c}^{\prime}$ increases by 1 , which results in an increased number of guards. But, if we dissect along $\overline{v_{c} v_{3}}$ then the line segment $\overline{v_{1} v_{3}}$ can be removed from $P_{1}$ (because it is guarded by the guard of $\left.v_{1}^{\prime} v_{3} v_{\mathrm{c}} v_{2} v_{\mathrm{d}}\right)$ and $\mathrm{P}_{\mathrm{b}}$ can be joined with the updated $\mathrm{P}_{\mathrm{c}}^{\prime}$. The size of $\left(\mathrm{P}_{\mathrm{b}} \cup \mathrm{P}_{\mathrm{c}}^{\prime} \cup\left\{v_{\mathrm{c}}\right\}\right) \backslash\left\{v_{4}^{\prime}\right\}$ is $3\left(k_{b}+k_{c^{\prime}}\right)+2$, so the number of guards of $P^{\prime}$ does not increase as a result of adjusting the partition. 
However, the current partition of $\mathrm{P}_{\mathrm{c}}^{\prime}$ may no longer be relevant and may require repartition as part of the polygon $\left(\mathrm{P}_{\mathrm{b}} \cup \mathrm{P}_{\mathrm{c}}^{\prime} \cup\left\{v_{\mathrm{c}}\right\}\right) \backslash\left\{v_{4}^{\prime}\right\}$.

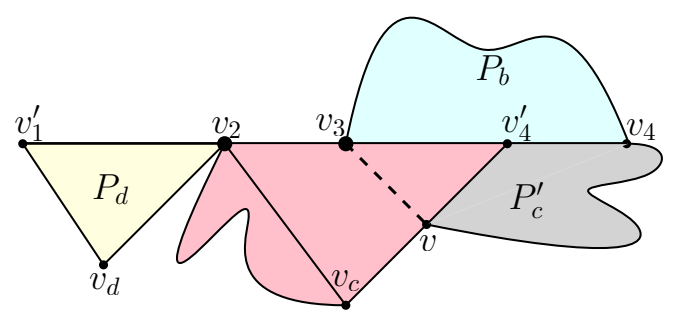

(a)

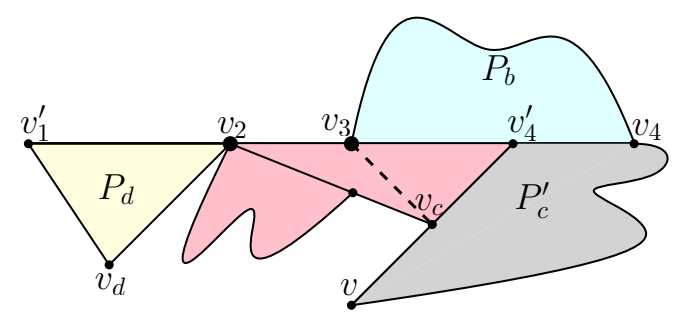

(b)

Figure 17.5: We avoid dissecting along $\overline{v_{\mathrm{c}} v_{4}^{\prime}}$.

Case 2.2: $\left|\mathrm{P}_{\mathrm{b}}\right|=3 \mathrm{k}_{\mathrm{b}}$. Then $\left|\mathrm{P}_{\mathrm{a}}\right|=3 \mathrm{k}_{\mathrm{a}}$. If $\left|\mathrm{P}_{\mathrm{c}}\right|=3 \mathrm{k}_{\mathrm{c}}$ and thus $\left|\mathrm{P}_{\mathrm{d}}\right|=3 \mathrm{k}_{\mathrm{d}}$ then $\overline{v_{2} v_{3}}$ is a good diagonal dissection, which is a contradiction. Thus either $\left|P_{c}\right|=3 k_{c}+1$ and $\left|P_{d}\right|=2$ or $\left|P_{d}\right|=3 k_{d}+1$ and $\left|P_{c}\right|=2$. Those cases are symmetrical to the the case 2.1.

\section{3 $n=3 k+2$ AND $\mathrm{P}^{\prime}$ HAS NO GOOD DIAGONAL DISSECTION}

In this section we assume that $n=3 k+2$ and every diagonal of $\mathrm{P}^{\prime}$ decomposes it into polygons of size $n_{1}=3 k_{1}$ and $n_{2}=3 k_{2}+1$, where $k_{1}+k_{2}=k+1$. Let $\mathcal{T}$ be a fixed triangulation of $P^{\prime}$, and let $G(\mathcal{T})$ be the dual graph of $\mathcal{T}$. Notice that $G(\mathcal{T})$ has $n-2$ nodes. (Later, during the algorithm, we may change this triangulation.)

The proofs of the following Propositions 4 and 5 are identical to those in [44]. We prove the following Lemmas 1,2 and 3 in this thesis.

то́тн's proposition 4. G(T) has exactly $k+1$ leaves.

то́тн'S LEMMA 1. If $\mathrm{P}^{\prime}$ has $\mathrm{n}=3 \mathrm{k}+2$ vertices and has no good dissection then $\mathrm{P}^{\prime}$ has at most $\mathrm{k}$ reflex angles.

TÓTH'S PROPOSITION 5. If $\mathrm{P}^{\prime}$ has at most $\mathrm{k}$ reflex angles, then $\mathrm{k} 180^{\circ}$-guards can monitor $\mathrm{P}^{\prime}$.

то́тн's LEMMA 2. If $\mathrm{P}^{\prime}$ has size $\mathrm{n}=3 \mathrm{k}+2$ then at least one of the following two statements is true:

1. $\mathrm{P}^{\prime}$ has a good dissection.

2. For every triangle $\triangle A B C$ in $\mathcal{T}$ that corresponds to a leaf of $\mathrm{G}(\mathcal{T})$ with $\mathrm{AC}$ being a diagonal of $\mathrm{P}^{\prime}$, either $\angle \mathrm{A}<\pi$ or $\angle \mathrm{C}<\pi$ in $\mathrm{P}^{\prime}$.

то́тH's LEMMA 3. If a convex angle of $\mathrm{P}^{\prime}$ is associated to two leaves in $\mathrm{G}(\mathcal{T})$, then $\left\lfloor\frac{\mathfrak{n}}{3}\right\rfloor 180^{\circ}$-guards can monitor $\mathrm{P}^{\prime}$.

\subsubsection{Adaptation of Tóth's Lemma 3 to our problem}

Let $v_{1}$ be a vertex at a convex angle of $\mathrm{P}^{\prime}$ associated to two leaves in $\mathrm{G}(\mathcal{T})$ (refer to Fig. 17.6). Let $\mathrm{P}_{1}$ be the polygon formed by all triangles of $\mathcal{T}$ adjacent to $v_{1}$. Notice that $P_{1}$ is a fan; $v_{1}$ is a center of the fan; and the dual of the triangulation of $\mathrm{P}_{1}$, inherited from $\mathrm{P}^{\prime}$, is a path of nodes. Recall that the center of a fan $P_{f}$ is a vertex of $P_{f}$ that can see all other vertices of $P_{f}$. 
The original proof of Tóth's Lemma 3 still holds for polygons not in general position. However, this is one of the places where a $180^{\circ}$-guard is explicitly positioned at $v_{1}$ to monitor all the triangles of $\mathcal{T}$ adjacent to $v_{1}$. If kernel $\left(P_{1}\right)$ is not a single point, then the positioning can be reused for our problem. We can put a pair of towers on the same edge of $\mathrm{P}^{\prime}$ in $\operatorname{kernel}\left(\mathrm{P}_{1}\right) \cap$ $\partial P_{1}$ to locate an agent in $P_{1}$. However, since there can be degenerate triangles among those adjacent to $v_{1}$, the kernel of $P_{1}$ can degenerate into a single point: $\operatorname{kernel}\left(\mathrm{P}_{1}\right)=v_{1}$. In this case, the location of an agent in $P_{1}$ cannot be determined with a pair of towers only. To overcome this problem we prove the following lemma.

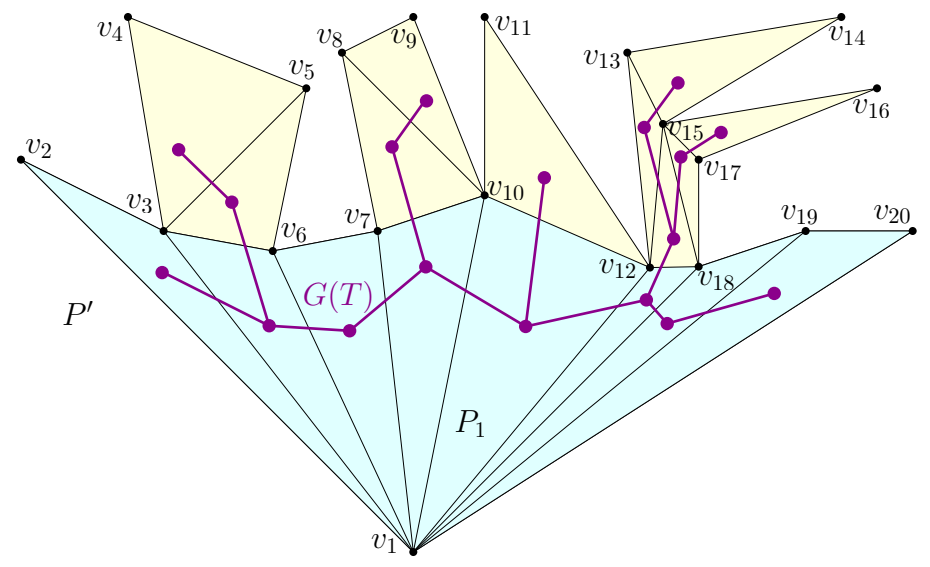

Figure 17.6: $\mathrm{P}^{\prime}$ has size $\mathrm{n}=20=3 \mathrm{k}+2$ for $\mathrm{k}=6$, and does not have good diagonal dissection. $G(\mathcal{T})$ is drawn on top of $\mathcal{T} . P_{1}$ is a fan with dominant point $v_{1}$ and it is highlighted in cyan.

LEMMA 17.3. There exist a triangulation of $\mathrm{P}^{\prime}$ such that its part, inherited by $\mathrm{P}_{1}$, does not contain any degenerate triangle.

Proof. Let us first observe that a triangle of $\mathcal{T}$, corresponding to a leaf in $\mathrm{G}(\mathcal{T})$, cannot be degenerate due to the simplification step performed on $\mathrm{P}^{\prime}$. There are two cases to consider:

Case 1: Suppose that the triangulation/fan of $P_{1}$ contains two or more degenerate triangles adjacent to each other. Let $v_{1}, u_{1}, u_{2}, \ldots, u_{i}$ for some $i>2$ be the vertices of the degenerate triangles sorted according to their distance from $v_{1}$. Notice that $v_{1}, u_{1}, u_{2}, \ldots, u_{i}$ belong to the same line. Those degenerate triangles must be enclosed in $\mathrm{P}_{1}$ between a pair of non-degenerate triangles, let us call them $\triangle_{1}$ and $\triangle_{2}$. Since $\mathrm{P}^{\prime}$ does not have angles of size $2 \pi$, the diagonal $\overline{v_{1} u_{1}}$ must be shared with one of $\triangle_{1}$ or $\triangle_{2}$. Assume, without loss of generality, that $u_{1}$ is a vertex of $\triangle_{1}$. Let $u_{j}$ be a vertex of $\triangle_{2}$, for some $1<j \leqslant i$. It is possible to re-triangulate $\mathrm{P}^{\prime}$ such that $\mathrm{P}_{1}$ will contain only one degenerate triangle $\triangle v_{1} u_{1} u_{j}$ between $\triangle_{1}$ and $\triangle_{2}$ and other triangles of $P_{1}$ (that are not between $\triangle_{1}$ and $\triangle_{2}$ ) will not be affected. The shape of $P_{1}$ may or may not change but its size will decrease.

Case 2: Suppose that the triangulation/fan of $P_{1}$ contains one degenerate triangle $\triangle v_{1} u_{1} u_{j}$ enclosed between a pair of non-degenerate triangles $\triangle_{1}$ and $\triangle_{2}$. Assume, without loss of generality, that $\triangle_{2}$ shares a diagonal $\overline{v_{1} u_{j}}$ with $\triangle v_{1} u_{1} u_{j}$. Let $w$ be the third vertex of $\triangle_{2}$, so $\triangle_{2}=\Delta v_{1} u_{j} w$. Notice that $u_{1}$ and $w$ see each other, because $u_{1}$ belongs to the line segment $\overline{v_{1} u_{j}}$. We can flip the diagonal $\overline{v_{1}} u_{j}$ into $\overline{u_{1} \mathcal{w}}$ in $\mathcal{T}$. As a result, $P_{1}$ will now contain $\triangle v_{1} u_{1} w$ instead of $\triangle v_{1} u_{1} u_{j}$ and $\triangle_{2}$. Notice that $\triangle v_{1} u_{1} w$ is non-degenerate.

We showed that we can obtain a triangulation of $\mathrm{P}^{\prime}$ in which all the triangles adjacent to $v_{1}$ are non-degenerate. Thus, $\mathrm{P}_{1}$ will have no degenerate triangles and still contain vertex $v_{1}$ together with two leaves of $\mathrm{G}(\mathcal{T})$ associated with $v_{1}$.

\subsubsection{Proof of Tóth's Lemma 2 and its adaptation to our problem}

This entire subsection is devoted to the proof of Tóth's Lemma 2.

Tóth defines two types of elements of $G(\mathcal{T})$. A leaf of $G(\mathcal{T})$ is called a short leaf if it is adjacent to a node of degree 3. If a leaf of $\mathrm{G}(\mathcal{T})$ is adjacent to a node of degree 2 then this leaf is called a long leaf. Since we are under the assumption that $n=3 k+2$ and $\mathrm{P}^{\prime}$ has no good diagonal dissection then the node of $\mathrm{G}(\mathcal{T})$ adjacent to a long leaf is also adjacent to a node of degree 3 . 
In this subsection we keep Tóth's original names and notations to simplify cross-reading.

The angle $\angle A B C$ is the angle that the ray $\overrightarrow{B A}$ makes while rotating counter-clockwise towards the ray $\overrightarrow{B C}$. For example, the angle of $P^{\prime}$ at $B$ is $\angle C B A$ (refer to Fig. 17.7).

Let $\triangle A B C$ correspond to a short leaf in $G(\mathcal{T})$, where $\overline{A C}$ is a diagonal of $\mathrm{P}^{\prime}$, and let $\triangle A C D$ correspond to a node in $G(\mathcal{T})$ adjacent to the leaf $\triangle A B C$. Refer to Fig. 17.7. Notice that $\triangle A B C$ cannot be degenerate, otherwise the vertex $B$ would be deleted during the simplification step. However, $\triangle A C D$ can be degenerate. The diagonals $\overline{A D}$ and $\overline{C D}$ decompose $\mathrm{P}^{\prime}$ into polygons $\mathrm{P}_{\mathrm{a}}, A B C D$ and $\mathrm{P}_{c}$, where $A \in \mathrm{P}_{\mathrm{a}}$ and $C \in P_{c}$. Let $n_{a}$ be size of $P_{a}$ and $n_{c}$ be size of $P_{c}$.

то́тH'S CLAIM 1. $\mathrm{n}_{\mathrm{a}}=3 \mathrm{k}_{\mathrm{a}}+1$ and $\mathrm{n}_{\mathrm{c}}=3 \mathrm{k}_{\mathrm{c}}+1$.

TótH's CLAIM 2. ABCD is a non-convex quadrilateral, i.e. it has a reflex vertex at $\mathrm{A}$ or $\mathrm{C}$.

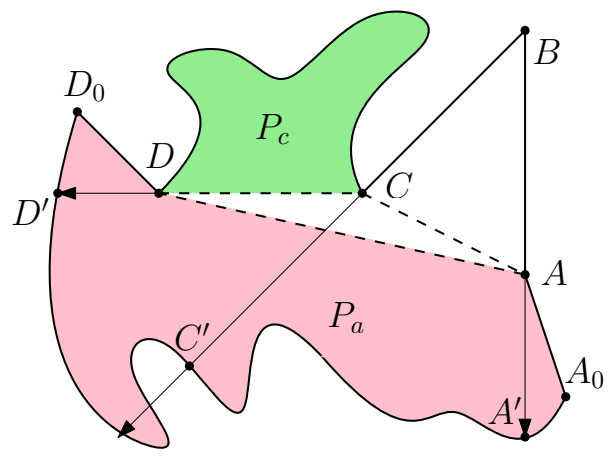

The original proof of both claims (refer to [44]) can be reused for polygons with vertices in non-general position.

Figure 17.7: $\triangle A B C$ corresponds to a short leaf in $G(\mathcal{T}) . \triangle A C D$ can be degenerate.

Assume, without loss of generality, that the reflex vertex of $A B C D$ is at $C$. Assume that $\mathcal{T}$ is the triangulation of $\mathrm{P}^{\prime}$ in which $\mathrm{n}_{\mathrm{a}}$ is minimal. This assumption together with the fact that $\mathrm{P}^{\prime}$ does not have a good diagonal dissection implies that there does not exist a vertex of $\mathrm{P}_{\mathrm{a}}$ in the interior of a line segment $\overline{D A}$. By Tóth's Claim $1, n_{a} \geqslant 4$ (notice that $n_{a} \neq 1$ because $A, D \in P_{a}$ ). Let $A_{0}$ and $D_{0}$ be vertices of $P_{a}$ adjacent to $A$ and $D$ respectively (refer to Fig. 17.7). By $\overrightarrow{u v}$ we denote the ray that starts at $u$ and passes through $v$. Let $A^{\prime}$ be a point such that $A^{\prime} \in \partial P^{\prime} \cap \overrightarrow{B A}$ and there exists a point $A^{\prime \prime} \in \partial P^{\prime}$ strictly to the right of $\overrightarrow{B A}$ such that $A^{\prime}$ and $A^{\prime \prime}$ belong to the same edge of $\mathrm{P}^{\prime}$ and both visible to $B$ and $A$. Let $D^{\prime}$ be a point such that $D^{\prime} \in \partial P^{\prime} \cap \overrightarrow{C D}$ and there exist a point $D^{\prime \prime} \in \partial P^{\prime}$ strictly to the left of $\overrightarrow{C D}$ such that $D^{\prime}$ and $D^{\prime \prime}$ belong to the same edge of $P^{\prime}$ and both visible to $C$ and $D$. Let $C^{\prime}$ be a point defined similarly to $D^{\prime}$ but with respect to the ray $\overrightarrow{B C}$. Notice that if $A=A^{\prime}$ then $\angle A=\angle B A A_{0}<\pi$ in $P^{\prime}$ and thus the second condition of Tóth's Lemma 2 holds. Therefore, assume that $A \neq A^{\prime}$.

то́тн's CLAIm 3. The points $\mathrm{A}^{\prime}$ and $\mathrm{D}^{\prime}$ belong to the same edge of $\mathrm{P}^{\prime}$.

Proof. Let us rotate the ray $\overrightarrow{C A^{\prime}}$ around $\mathrm{C}$ in the direction of $\mathrm{D}^{\prime}$. Notice that in the original proof, Tóth uses $\overrightarrow{C A}$. However, there are polygons (even in general position) for which Tóth's proof does not hold. For example, when the ray $\overrightarrow{C A}$ hits $A_{0}$, then Tóth claims that $\angle B A A_{0}<\pi$. Figure 17.7 can serve as a counterexample to this claim, because $A_{0}$ is indeed the first point hit by the rotating ray $\overrightarrow{C A}$, however $\angle B A A_{0}>\pi$.

The structure of the original proof can be used with respect to $\overrightarrow{C A^{\prime}}$, assuming that $A^{\prime}$ is visible to C. Thus, assume first that $C$ and $A^{\prime}$ can see each other. We rotate $\overrightarrow{C A^{\prime}}$ around $C$ in the direction of $D^{\prime}$. Let $\mathrm{O}$ be the first vertex of $\mathrm{P}^{\prime}$ visible from $\mathrm{C}$ that was hit by the ray (in case there are several collinear such vertices of $\mathrm{P}_{\mathrm{a}}$, then let $\mathrm{O}$ be the one that is closest to $\mathrm{C}$ ).

If $\mathrm{O}=\mathrm{D}$ then $\mathrm{A}^{\prime}$ and $\mathrm{D}^{\prime}$ belong to the same edge of $\mathrm{P}^{\prime}$ (notice that it is possible that $\mathrm{D}^{\prime}=\mathrm{D}$ ), so the claim holds.

If $\mathrm{O}=\mathrm{A}_{0}$ then $A=A^{\prime}, \angle A=\angle B A A_{0}<\pi$ and thus the second claim in Tóth's Lemma 2 holds.

If $O \neq D$ and $O \neq A_{0}$, then $\overline{A O}$ and $\overline{C O}$ are diagonals of $P^{\prime}$. Refer to Fig. 17.8a. There exists a triangulation of $\mathrm{P}^{\prime}$ that contains $\triangle A C O$ and has $\triangle A B C$ as a short leaf. Consider quadrilateral $A B C O$. It is non-convex by Tóth's Claim 2. By construction, $\mathrm{O}$ is to the right of $\overrightarrow{\mathrm{BA}}$, thus $\angle \mathrm{BAO}<\pi$. It follows, that the only possible reflex angle in $A B C O$ is $\angle O C B$, which is a contradiction to the minimality of $P_{a}$, and thus, such a vertex $O$ does not exist. 
Assume now that $A^{\prime}$ is not visible to $C$. It follows that some part of $\partial \mathrm{P}^{\prime}$ belongs to the interior of $\triangle A A^{\prime} C$. There must be a vertex of $P_{a}$ interior to $\triangle A A^{\prime} C$, otherwise there is inconsistency with the construction of $A^{\prime}$. Moreover, among all the vertices of $P_{a}$ interior to $\triangle A A^{\prime} C$, there must be at least one that is visible to $\mathrm{B}$. Let us call it $\mathrm{O}^{\prime}$. Notice that $\overline{\mathrm{AO}^{\prime}}$ and $\overline{\mathrm{CO}^{\prime}}$ are diagonals of $\mathrm{P}^{\prime}$. The quadrilateral $A B C A^{\prime}$ is convex, which contradicts Tóth's Claim 2. Thus, $A^{\prime}$ is visible to $C$.

It follows from Tóth's Claim 3 that the quadrilateral $\mathrm{A}^{\prime} \mathrm{BCD}^{\prime}$ has no common points with $\partial \mathrm{P}^{\prime}$ in its interior, but on the boundary only.

We have derived several properties satisfied by $\mathrm{P}^{\prime}$ and now we are ready to show the existence of good (non-diagonal) dissections that consist of one or two line segments. We discuss the following three cases, that span over Claims 4,5 in [44].

Case 1: $\angle D_{0} D C<\pi$. In this case $D=D^{\prime}$. It follows from Tóth's Claim 3 that $A^{\prime}, C^{\prime} \in \overline{D^{2}}$. Refer to Fig. $17.8 \mathrm{~b}$.

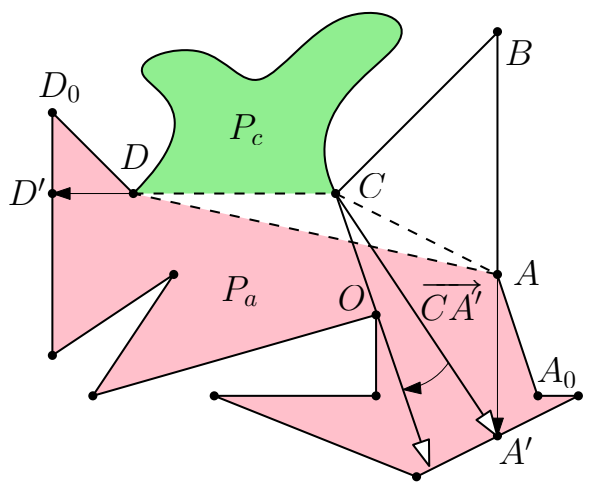

(a)

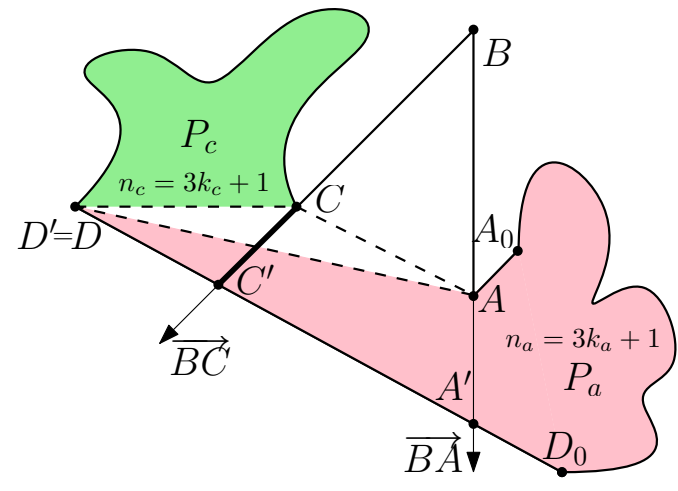

(b)

Figure 17.8: $\triangle A B C$ corresponds to a short leaf in $G(\mathcal{T}) . \triangle A C D$ can be degenerate. (a) $\angle D_{0} D C>\pi$. (b) $\angle D_{0} \mathrm{DC}<\pi$. $\overline{\mathrm{C} \mathrm{C}^{\prime}}$ is a good dissection.

Line segment $\overline{C C^{\prime}}$ represents a good dissection that splits $P^{\prime}$ into two polygons: $P_{c} \cup \triangle C C^{\prime} D$ of size $n_{c}+1=3 k_{c}+2$ and $\left(P^{\prime} \backslash P_{c}\right) \backslash \triangle C C^{\prime} D$ of size $n_{a}+1=3 k_{a}+2$. Notice that $\overline{B C^{\prime}}$ is an edge of the subpolygon of $\mathrm{P}^{\prime}$ to the left of $\overrightarrow{B C}$ and thus $C$ is not a vertex of this subpolygon. If $\triangle A C D$ is degenerate (in which case $C$ is between $A$ and $D$ ), then $\overline{C C^{\prime}}$ is still a good dissection. However, if $D_{0}$ is also collinear with $D, C$ and $A$, then $D_{0}$ is visible to $C$ and $\overline{C D_{0}}$ represents a good diagonal dissection.

Case 2: $\angle \mathrm{D}_{0} \mathrm{DC}=\pi$. In this case $\mathrm{D}_{0}=\mathrm{D}^{\prime}$ and $\overline{\mathrm{CD}}$ is a good diagonal dissection that splits $\mathrm{P}^{\prime}$ into $P_{c}$ of size $n_{c}=3 k_{c}+1$ and $P_{a} \cup A B C D$ of size $n_{a}+1=3 k_{a}+2$. Notice that $\overline{C D_{0}}$ is an edge in $P_{a} \cup A B C D$ and thus $D$ is not a vertex in $P_{a} \cup A B C D$.

Case 3: $\angle D_{0} D C>\pi$. Let $D_{0}^{\prime}$ be the point closest to $D$ where the ray $\overrightarrow{D_{0} D}$ reaches $\partial P^{\prime}$. If the line segments $\overline{\mathrm{CC}^{\prime}}$ and $\overline{\mathrm{DD}_{0}^{\prime}}$ intersect inside the quadrilateral $C A A^{\prime} \mathrm{D}^{\prime}$ at $\mathrm{Q}$ (refer to Fig. 17.9a), then $\overline{\mathrm{DQ}} \cup \overline{\mathrm{QC}}$ is a good dissection, that splits $\mathrm{P}^{\prime}$ into polygon $\mathrm{P}_{\mathrm{c}} \cup \triangle \mathrm{CDQ}$ of size $n_{\mathrm{c}}+1=3 \mathrm{k}_{\mathrm{c}}+2$ and polygon $P_{a} \cup D Q B A$ of size $n_{a}+1=3 k_{a}+2$.

However, if $C A A^{\prime} D^{\prime}$ degenerates into a line segment (which happens when $\triangle A C D$ is degenerate and there exists an edge $\overline{\mathrm{IJ}}$ of $\mathrm{P}_{\mathrm{a}}$ that contains $\overline{\mathrm{AD}}$ ) then $\mathrm{Q}$ cannot be defined. Refer to Fig. 17.9b. In this case we show that $\mathrm{P}^{\prime}$ has a good diagonal dissection. Notice that $\overline{\mathrm{ID}}$ is a diagonal of $\mathrm{P}_{\mathrm{a}}$; it splits $P_{a}$ into two subpolygons. Let $P_{a}^{\prime}$ be a subpolygon of $P_{a}$ that contains $D_{0}$. Let $n_{a}^{\prime}$ be the size of $P_{a}^{\prime}$. We consider three cases:

- $\mathrm{n}_{\mathrm{a}}^{\prime}=3 \mathrm{k}_{\mathrm{a}}^{\prime}$ : In this case $\overline{\mathrm{IA}}$ is a good diagonal dissection. The size of $\mathrm{P}_{\mathrm{a}}^{\prime} \cup \mathrm{P}_{\mathrm{c}} \cup \triangle A B C$ is $3 k_{\mathrm{a}}^{\prime}+$ $3 k_{c}+1+3-2=3\left(k_{a}^{\prime}+k_{c}\right)+2$. Notice that the " -2 " in the previous formula stands for vertices $\mathrm{D}$ and $\mathrm{C}$ that were counted twice. 


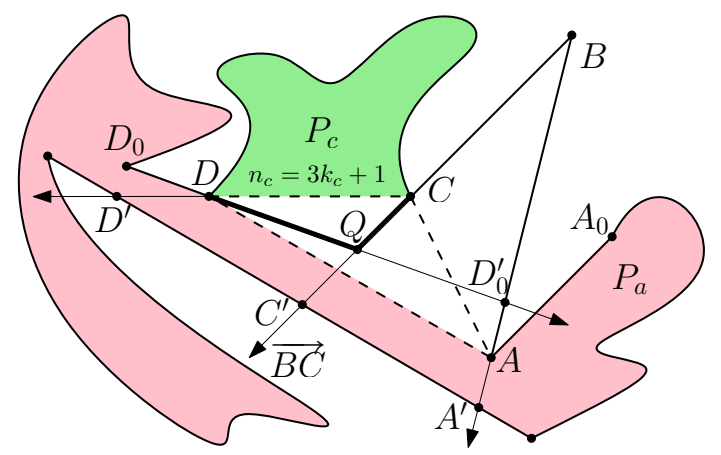

(a)

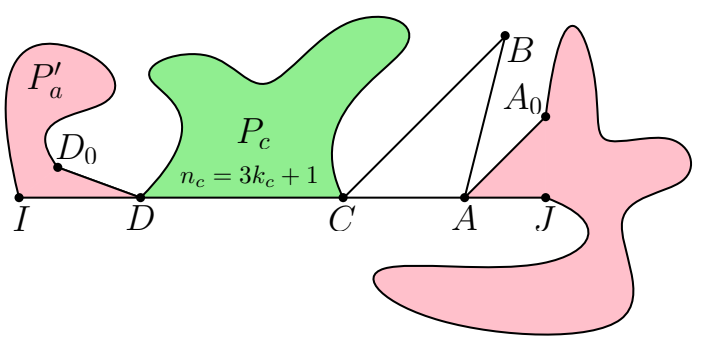

(b)

Figure 17.9: $\triangle A B C$ corresponds to a short leaf in $\mathrm{G}(\mathcal{T}) ; \angle D_{0} D C>\pi$. (a) $\overline{\mathrm{DQ}} \cup \overline{\mathrm{QC}}$ is a good dissection of $\mathrm{P}^{\prime}$. (b) $\triangle A C D$ is degenerate; the edge $\overline{\mathrm{IJ}}$ of $\mathrm{P}_{\mathrm{a}}$ contains $\overline{\mathrm{DA}} . \mathrm{P}_{\mathrm{a}}$ is highlighted in pink. Notice that $C$ is not a vertex of $P_{a} . P_{a}^{\prime}$ is a subpolygon of $P_{a}$ that contains $D_{0}$ and $\overline{I D}$ is its edge.

- $\mathrm{n}_{\mathrm{a}}^{\prime}=3 \mathrm{k}_{\mathrm{a}}^{\prime}+1$ : In this case $\overline{\mathrm{CJ}}$ is a good diagonal dissection. The size of $\mathrm{P}_{\mathrm{a}}^{\prime} \cup \mathrm{P}_{\mathrm{c}} \cup$ IDCJ is $3 k_{a}^{\prime}+1+3 k_{c}+1+1-1=3\left(k_{a}^{\prime}+k_{c}\right)+2$.

- $\mathrm{n}_{\mathrm{a}}^{\prime}=3 \mathrm{k}_{\mathrm{a}}^{\prime}+2$ : In this case $\overline{\mathrm{ID}}$ is a good diagonal dissection.

In this subsection we assumed that $\mathrm{P}^{\prime}$ has no good diagonal dissection, thus we deduce that $C A A^{\prime} D^{\prime}$ cannot degenerate into a line segment. Notice that $\mathrm{Q}$ exists even when $\triangle A C D$ is degenerate.

If $\overline{\mathrm{CC}^{\prime}}$ and $\overline{\mathrm{DD}_{0}^{\prime}}$ do not intersect inside $C A A^{\prime} D^{\prime}$, then $D_{0}^{\prime}$ belongs to the line segment $\mathrm{C}^{\prime} \mathrm{D}^{\prime}$. Refer to Fig. 17.10. Tóth shows in Claim 5 in [44] that one of the line segments $\overline{\mathrm{DD}_{0}^{\prime}}, \overline{\mathrm{CC}^{\prime}}$ and $\overline{A A^{\prime}}$ is a good dissection.

It was shown so far that if $\triangle A B C$ is a short leaf in $G(\mathcal{T})$, then either $P^{\prime}$ has a good dissection or the angle at vertex $A$ or $C$ in $P^{\prime}$ is convex. It is left to prove that Tóth's Lemma 2 is true for long leaves. Notice that if $\triangle A B C$ is a long leaf in $G(\mathcal{T})$ but there exists a triangulation of $P^{\prime}$ where $\triangle A B C$ is a short leaf then Tóth's Lemma 2 is true for $\triangle A B C$.

Let $\triangle A B C$ be a long leaf of $G(\mathcal{T})$ such that there does not exist a triangulation of $\mathrm{P}^{\prime}$ where $\triangle A B C$ is a short leaf. Recall that in this subsection, we assumed that $\mathrm{P}^{\prime}$ has no good diagonal

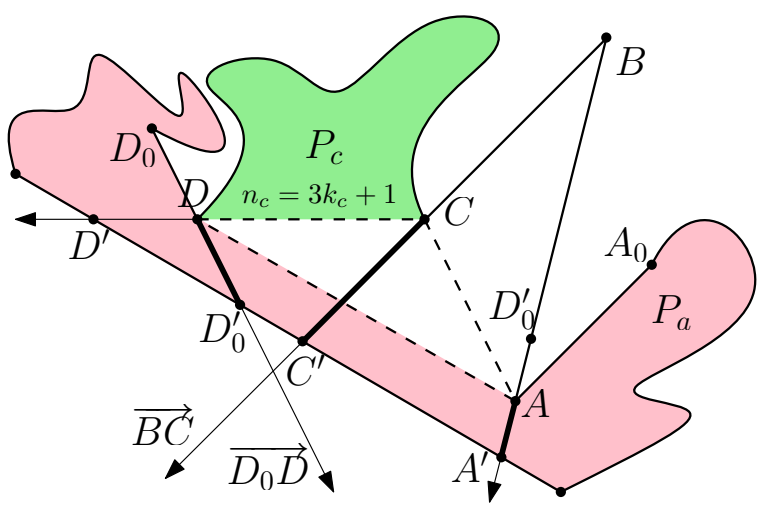

Figure 17.10: $\triangle A B C$ corresponds to a short leaf in $\mathrm{G}(\mathcal{T}) ; \angle \mathrm{D}_{0} \mathrm{DC}>\pi ; \triangle A C D$ can be degenerate. One of $\overline{\mathrm{DD}_{0}^{\prime}}, \overline{\mathrm{CC}^{\prime}}$ and $\overline{\mathrm{AA}^{\prime}}$ is a good dissection of $\mathrm{P}^{\prime}$. dissection. Thus, the node of $\mathrm{G}(\mathcal{T})$ adjacent to a long leaf is also adjacent to a node of degree 3. We also concluded that $\triangle A B C$ cannot be degenerate.

то́тн's CLAIM 6. If $\triangle A B C$ is a long leaf of $\mathrm{G}(\mathcal{T})$ for every triangulation $\mathcal{T}$ of $\mathrm{P}^{\prime}$ then the node of $\mathrm{G}(\mathcal{T})$ adjacent to the node $\triangle \mathrm{ABC}$ corresponds to the same triangle for every $\mathcal{T}$.

Tóth's Claim 6 is true for a triangulation $\mathcal{T}$ that contains degenerate triangles and thus it is true for the $\mathrm{P}^{\prime}$ defined in this thesis.

Let $\triangle A C D$ be a triangle adjacent to $\triangle A B C$ in $\mathcal{T}$. The ray $\overrightarrow{C A}$ (respectively $\overrightarrow{C D}, \overrightarrow{B C}, \overrightarrow{B A}$ ) reaches $\partial P^{\prime}$ at $A^{\prime}$ (respectively $D^{\prime}, C^{\prime}, B^{\prime}$ ). Notice that $A^{\prime}$ is defined differently than in the case with short 
leaves. Refer to Fig. 17.11. By Tóth's Claim 6, $\triangle A C D$ is unique. Notice that $\triangle A C D$ can be degenerate, but because it is unique, it does not contain any other vertex of $\mathrm{P}^{\prime}$. Moreover, there does not exist an edge $\overline{\mathrm{IJ}}$ of $\mathrm{P}^{\prime}$ that contains $\triangle A C D$, otherwise $\mathrm{P}^{\prime}$ has a good diagonal dissection since $A, C, D, I$ and J can see each other. Tóth's Claim 7, that follows this discussion, is thus true for polygons whose triangulation may contain degenerate triangles.

то́тH'S CLAIM 7. The points $\mathrm{A}^{\prime}$ and $\mathrm{D}^{\prime}$ belong to the same edge of $\mathrm{P}^{\prime}$ or the angle of $\mathrm{P}^{\prime}$ at $\mathrm{A}$ is convex and thus the second condition of Tóth's Lemma 2 holds for $\triangle \mathrm{ABC}$. Refer to Fig. 17.11.

It follows that $C^{\prime}$ and $B^{\prime}$ belong to the same edge as $A^{\prime}$ and $D^{\prime}$.

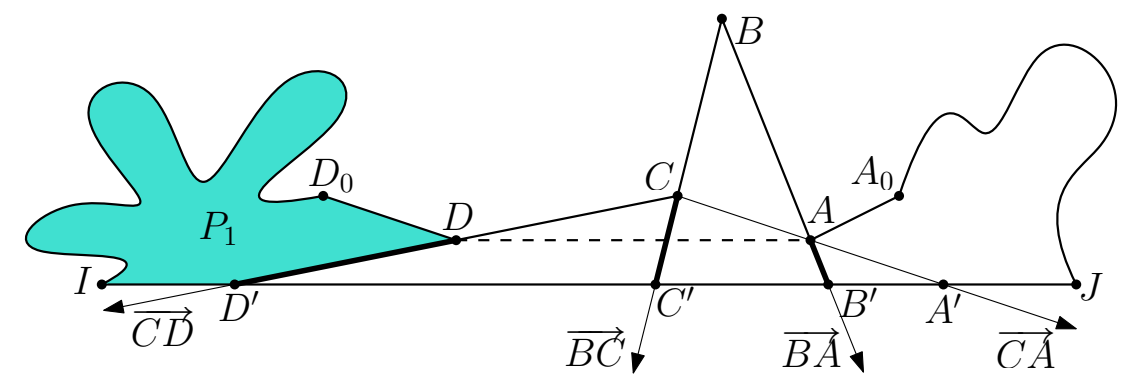

Figure 17.11: $\triangle A B C$ corresponds to a long leaf in $G(\mathcal{T}) . \overline{\mathrm{IJ}}$ is an edge of $\mathrm{P}^{\prime}$; it contains $\mathrm{A}^{\prime}, \mathrm{B}^{\prime}, \mathrm{C}^{\prime}$ and $\mathrm{D}^{\prime}$. One of $\overline{\mathrm{DD}^{\prime}}, \overline{\mathrm{CC}^{\prime}}$ or $\overline{\mathrm{AB}^{\prime}}$ is a good dissection of $\mathrm{P}^{\prime}$. $\mathrm{P}_{1}$ is highlighted in cyan.

Assume that the angles of $\mathrm{P}^{\prime}$ at $A$ and $C$ are reflex, otherwise the second condition of Tóth's Lemma 2 holds for $\triangle A B C$ and our proof is complete.

Consider the angle of $\mathrm{P}^{\prime}$ at $\mathrm{D}$. It can be either convex or reflex. Notice that non of the angles of $\mathrm{P}^{\prime}$ equals $\pi$ or 0 because of the simplification step. We discuss the following two cases, that span over Claims 8,9 in [44], and show that in either case $\mathrm{P}^{\prime}$ has a good dissection.

Case 1: $\angle D_{0} D C>\pi$. One of the line segments $\overline{\mathrm{DD}^{\prime}}, \overline{C C^{\prime}}$ or $\overline{\mathrm{AB}^{\prime}}$ is a good dissection of $\mathrm{P}^{\prime}$. Refer to Fig. 17.11. $\overline{\mathrm{DD}^{\prime}}$ partitions $\mathrm{P}^{\prime}$ into two subpolygons. Let $\mathrm{P}_{1}$ be one of them that contains $\mathrm{D}_{0}$ (it is highlighted in cyan on Fig. 17.11). The size of $P_{1}$ is $n_{1}=3 k_{1}+q_{1}$; the size of $P_{1} \cup C^{\prime} D^{\prime} C^{\prime}$ is $n_{1}+1$; the size of $P_{1} \cup C D D^{\prime} C^{\prime} \cup A C C^{\prime} B^{\prime}$ is $n_{1}+2$. If $q_{1}=2$ (respectively $q_{1}=1, q_{1}=0$ ) then $\overline{D D^{\prime}}$ (respectively $\overline{C C^{\prime}}, \overline{A B^{\prime}}$ ) is a good dissection of $\mathrm{P}^{\prime}$.

Case 2: $\angle D_{0} D C<\pi$. It follows that $D^{\prime}=D, A^{\prime} \in \overline{D_{0}}$ and $A^{\prime} \neq D_{0}$, otherwise $\overline{A_{0}}$ is a good diagonal dissection. Refer to Fig. 17.12. Let $A_{0}^{\prime}$ be the point where $\overrightarrow{A_{0} A}$ reaches $\partial P^{\prime}$. Tóth's Claim 7 implies that $A_{0}^{\prime} \in \overline{\mathrm{CD}}$ or $A_{0}^{\prime} \in \overline{\mathrm{DB}^{\prime}}$. If $A_{0}^{\prime} \in \overline{\mathrm{DB}^{\prime}}$ (refer to Fig. 17.12a) then $\overline{\mathrm{AA}_{0}^{\prime}}$ is a good dissection of $P^{\prime}$. It creates a pentagon $A B C D A_{0}^{\prime}$ and a polygon $P^{\prime} \backslash A B C D A_{0}^{\prime}$ whose size is $n-3=3(k-1)-2$ (notice that $\overline{A A_{0}^{\prime}}$ is an edge of $P^{\prime} \backslash A B C D A_{0}^{\prime}$ and thus $A \notin P^{\prime} \backslash A B C D A_{0}^{\prime}$ ). Notice that if $A_{0}^{\prime}=D$ then $A_{0}$ can see $D$ and thus $\overline{A_{0} D}$ is a good diagonal dissection of $P^{\prime}$.

Assume that $A_{0}^{\prime} \in \overline{C D}$. Refer to Fig. 17.12b. Let us assign labels to the vertices of $\mathrm{P}^{\prime}$ according to their order around $\partial \mathrm{P}^{\prime}$ as follows: $v_{0}=A, v_{1}=A_{0}, v_{2}, \ldots, v_{n-4}=D_{0}, v_{n-3}=D, v_{n-2}=C$, $v_{n-1}=B . \overline{D A}$ is a diagonal of $P^{\prime}$ and thus at least some interval of $\overline{A A_{0}}$ is visible to $D$. Let us rotate $\overrightarrow{D A}$ towards $A_{0}$. The ray hits $v_{i}$ for $1<i \leqslant v_{n-5}$ (notice that the ray cannot hit $A_{0}$, otherwise $\overrightarrow{D A_{0}}$ is a good diagonal dissection). Observe that the angle of $\mathrm{P}^{\prime}$ at $v_{i}$ must be reflex. Let $X_{1}$ (respectively $X_{2}$ ) be a point where $\overrightarrow{v_{i+1} v_{i}}$ (respectively $\overrightarrow{v_{i-1} v_{i}}$ ) reaches $\partial \mathrm{P}^{\prime}$. By construction, $X_{1} \in \overline{A A_{0}}$ or $X_{1} \in \overline{C D}$. The same is true for $X_{2}$. If $i$ is not a multiple of 3 then one of $\overline{v_{i} X_{1}}$ or $\overline{v_{i} X_{2}}$ is a good dissection.

- $i \equiv 1 \bmod 3:$

- $\mathrm{X}_{1} \in \overline{\mathrm{AA}}$. Then the subpolygon $v_{1} v_{2} \ldots v_{\mathrm{i}} \mathrm{X}_{1}$ has size $3 k^{\prime}+2$, and the subpolygon $A X_{1} v_{i+1} v_{i+2} \ldots v_{n-1}$ has size $3 k^{\prime \prime}+2$ for some $k^{\prime}+k^{\prime \prime}=k($ recall that $n=3 k+2)$. 


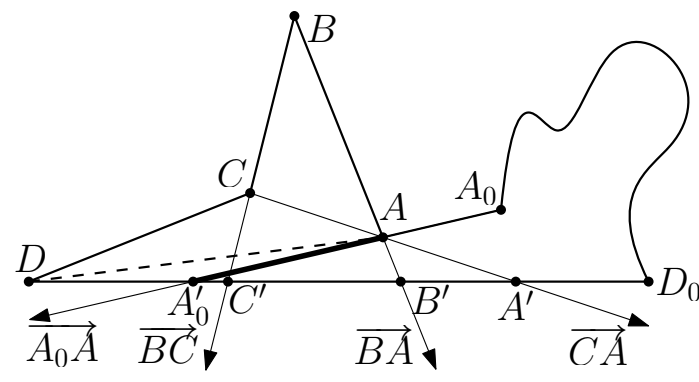

(a)

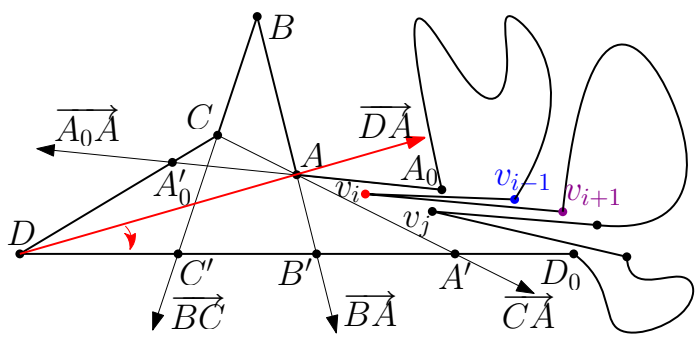

(b)

Figure 17.12: $\triangle A B C$ corresponds to a long leaf in $G(\mathcal{T}) ; \angle D_{0} D C<\pi$. (a) Case where $A_{0}^{\prime} \in \overline{D^{\prime}}$. $\overline{\mathrm{AA}_{0}^{\prime}}$ is a good dissection of $P^{\prime}$. (b) Case where $A_{0}^{\prime} \in \overline{C D}$.

- $\mathrm{X}_{1} \in \overline{\mathrm{CD}}$. Then the polygon $\mathrm{CBA} v_{1} v_{2} \ldots v_{\mathrm{i}} \mathrm{X}_{1}$ has size $3 \mathrm{k}^{\prime}+2$, and the subpolygon $X_{1} v_{i+1} v_{i+2} \ldots v_{n-3}$ has size $3 k^{\prime \prime}+2$ for some $k^{\prime}+k^{\prime \prime}=k$.

- $i \equiv 2 \bmod 3$ :

- $\mathrm{X}_{2} \in \overline{\mathrm{AA}_{0}}$. Then the polygon $v_{1} v_{2} \ldots v_{i-1} \mathrm{X}_{2}$ has size $3 \mathrm{k}^{\prime}+2$, and the subpolygon $A X_{2} v_{i} v_{i+1} \ldots v_{n-1}$ has size $3 k^{\prime \prime}+2$ for some $k^{\prime}+k^{\prime \prime}=k$.

- $\mathrm{X}_{2} \in \overline{\mathrm{CD}}$. Then the polygon $\mathrm{CBA} v_{1} v_{2} \ldots v_{i-1} \mathrm{X}_{2}$ has size $3 k^{\prime}+2$, and the subpolygon $\mathrm{X}_{2} v_{i} v_{i+1} \ldots v_{n-3}$ has size $3 k^{\prime \prime}+2$ for some $k^{\prime}+k^{\prime \prime}=k$.

If $i$ is a multiple of 3 then we repeat the above procedure and rotate the ray $\overrightarrow{D v_{i}}$ towards $v_{i+1}$. Notice that if the ray hits $v_{i+1} \neq \mathrm{D}_{0}$ then $\overline{\mathrm{D} v_{i+1}}$ is a good diagonal dissection (which is a contradiction to our main assumption). Thus the ray hits $v_{j}$ for $i+1<j \leqslant v_{n-5}$. If $j$ is not a multiple of 3 then one of the rays $\overrightarrow{v_{j+1} v_{j}}$ or $\overrightarrow{v_{j-1} v_{j}}$ contains a good dissection. Observe that those rays reach $\partial \mathrm{P}^{\prime}$ at $\overline{\mathrm{CD}}, \overline{\mathrm{AA}} \overline{0}$ or $\overline{v_{i} v_{i+1}}$. Since we perform counting modulo 3, those edges are considered to be identical in terms of vertices' indices. It means that we do not have to know where exactly $\overrightarrow{v_{j+1} v_{j}}$ or $\overrightarrow{v_{j-1} v_{j}}$ reach $\partial P^{\prime}$ to decide which dissection to apply. That is, if $j \equiv 1 \bmod 3$ then we use $\overrightarrow{v_{j+1} v_{j}}$; if $j \equiv 2 \bmod 3$ then we use $\overrightarrow{v_{j-1} v_{j}}$.

If $j$ is a multiple of 3 then the procedure is repeated again. Eventually, the ray spinning around $D$ must hit $D_{0}$. Recall that $D_{0}=v_{n-4} ; n-4=3 k+2-4=3(k-1)+1$ which is not a multiple of 3 . At this point Tóth comes to a contradiction and states that the angle of $\mathrm{P}^{\prime}$ at $\mathrm{D}$ cannot be convex (refer to Claim 9 in [44]). However there is no contradiction. We show that the situation is possible and discuss how to find a good dissection in this case.

Let $v_{z}$ be the last vertex hit by the ray spinning around $\mathrm{D}$ before it hit $\mathrm{D}_{0}$. Notice that $z$ is a multiple of 3. Two cases are possible:

1. $v_{z+1} \neq \mathrm{D}_{0}$ : notice that $\mathrm{D}_{0}$ can see $v_{z}$. The size of the subpolygon $v_{z} v_{z+1} \ldots v_{n-4}$ is $3 k^{\prime}+2$ for some integer $k^{\prime}>1$ ( $k$ is strictly bigger than 1 because $v_{z+1} \neq \mathrm{D}_{0}$ ). Therefore, the diagonal $\overline{v_{z} \mathrm{D}_{0}}$ is a good diagonal dissection, meaning that this case is not possible.

2. $v_{z+1}=\mathrm{D}_{0}$ : in this case $z=\mathrm{n}-5$ and the angle of $\mathrm{P}^{\prime}$ at $\mathrm{D}_{0}$ is convex. Refer to Fig. 17.13.

- If $v_{z}$ can see $A$ then dissect $\mathrm{P}^{\prime}$ along $\overline{v_{z} \mathrm{~A}}$. This dissection creates two subpolygons: $A v_{z} \mathrm{D}_{0} \mathrm{DCB}$ of size 6 and $A v_{1} v_{2} \ldots v_{z}$ of size $n-4=3(k-1)+1$. Notice that the hexagon $A v_{z} D_{0} D C B$ has a non-empty kernel whose intersection with the boundary of $A v_{z} D_{0} D C B$ is $\overline{C^{\prime} B^{\prime}}$. One $180^{\circ}$-guard on $\overline{\mathrm{C}^{\prime} \mathrm{B}^{\prime}}$ can monitor $\mathrm{A} v_{z} \mathrm{D}_{0} \mathrm{DCB}$. Similarly, for our problem, two distinct towers on $\overline{\mathrm{C}^{\prime} \mathrm{B}^{\prime}}$ can localise an agent in $A v_{z} \mathrm{D}_{0} \mathrm{DCB}$ (notice that $\mathrm{C}^{\prime} \neq \mathrm{B}^{\prime}$ and thus $\overline{\mathrm{C}^{\prime} \mathrm{B}^{\prime}}$ contains at least two distinct points).

- If $v_{z}$ can not see $A$ then consider the ray $\overrightarrow{D_{0} v_{z}}$. Let $Z$ be a point where $\overrightarrow{D_{0} v_{z}}$ reaches $\partial \mathrm{P}$ or $\overline{\mathrm{AA}^{\prime}}$ (whichever happens first). 
- If $Z \in \overline{A A^{\prime}}$ (refer to Fig. 17.13a) then dissect $P^{\prime}$ along the two line segments $\overline{A Z} \cup$ $\bar{Z} v_{z} . P^{\prime}$ falls into two subpolygons: $A Z v_{z} D_{0} D C B$ of size 7 and $Z A v_{1} v_{2} \ldots v_{z}$ of size $n-3=3(k-1)+2$. Similarly to the hexagon from the previous case, the heptagon $\mathrm{AZ} v_{z} \mathrm{D}_{0} \mathrm{DCB}$ can be guarded by one $180^{\circ}$-guard on $\overline{\mathrm{C}^{\prime} \mathrm{B}^{\prime}}$ and our agent can be localised by a pair of distinct towers positioned on $\overline{C^{\prime} B^{\prime}}$.

- If $Z \notin \overline{A A^{\prime}}$ (refer to Fig. 17.13b) then there must be a vertex $v_{x}$ to the left of $\overrightarrow{\mathrm{AA}^{\prime}}$ visible to $D_{0}$ and to $D$. It follows that $x$ is a multiple of 3 . Thus $\overline{v_{x} D_{0}}$ is a good diagonal dissection. It means that this case is not possible.

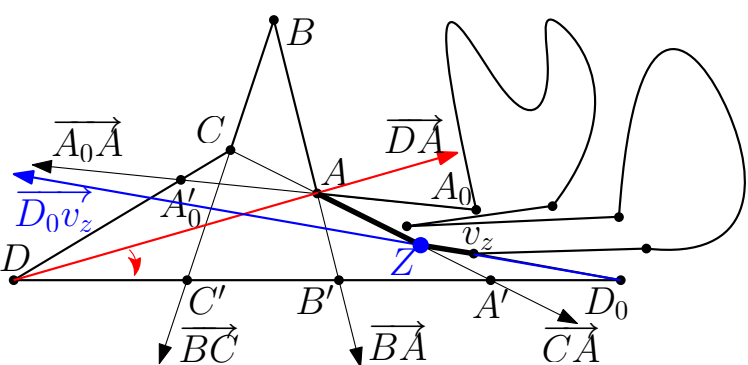

(a)

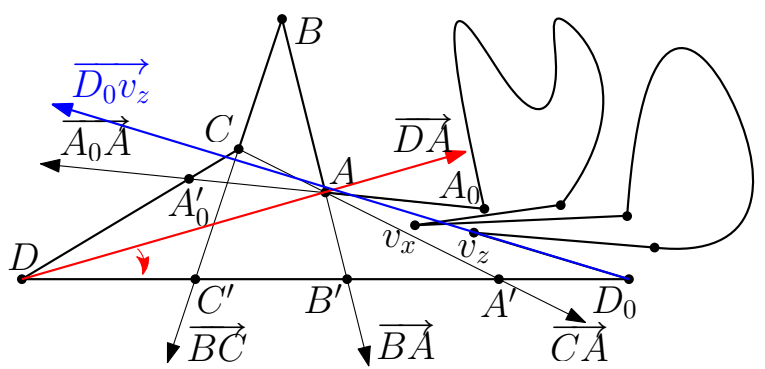

(b)

Figure 17.13: $\triangle A B C$ corresponds to a long leaf in $\mathrm{G}(\mathcal{T}) ; \angle D_{0} D C<\pi ; A_{0}^{\prime} \in \overline{\mathrm{CD}}$. (a)Two line segments $\overline{A Z} \cup \overline{Z v_{z}}$ is a good dissection. (b) Impossible case, because $\overline{v_{x} D_{0}}$ is a good diagonal dissection.

This completes the proof of Tóth's Lemma 2.

\subsection{PARTITION ALGORITHM}

We are given a simple polygon $P$ in general position of size $n=3 k+q$ where $k$ is a positive integer and $q \in\{0,1,2\}$. If $P$ has at most $k$ reflex angles then $P$ can be partitioned simply by bisecting $k-1$ of the reflex angles. This creates $k$ star-shaped subpolygons of $P$ that can be watched by $2 k$ towers. If the number of reflex angles of $\mathrm{P}$ is bigger than $\mathrm{k}$ then we look for a good diagonal dissection. In Section 17.2 we modified Tóth's partition [44] to deal with subpolygons of $P$ that are not in general position. The important difference to notice is that we avoided dissecting along diagonals of $\mathrm{P}^{\prime}$ that contain vertices of $\mathrm{P}$ in their interior. If the dissection is unavoidable we showed how to position towers and in the worst case - repartition subpolygons of $\mathrm{P}^{\prime}$.

If $\mathrm{P}^{\prime}$ has no good diagonal dissection then its size is $n=3 k+2$. In this case we look for a good dissection via the short or long leaf approach discussed in Section 17.3.2. If no good cut is found then by Tóth's Lemma 2 every leaf in $\mathrm{G}(\mathcal{T})$ is associated to two convex angles of $\mathrm{P}^{\prime}$. It follows by Tóth's Lemma 3 that $P^{\prime}$ can be monitored by $\left\lfloor\frac{n}{3}\right\rfloor 180^{\circ}$-guards. Refer to Section 17.3.I on how to adapt Tóth's Lemma 3 for tower positioning. We refer to Tóth's Lemma 1 to show the coherence of the algorithm. Tóth's Lemma 1 states that if $\mathrm{P}^{\prime}$ has $\mathrm{n}=3 \mathrm{k}+2$ vertices and has no good diagonal or other dissection then $\mathrm{P}^{\prime}$ has at most $\mathrm{k}$ reflex angles and thus $\mathrm{P}^{\prime}$ would be treated during the first step of the algorithm.

The obtained partition together with the locations of $180^{\circ}$-guards is reused for tower positioning. Every $180^{\circ}$-guard that guards subpolygon $\mathrm{P}^{\prime}$ is positioned on the boundary of $\mathrm{P}^{\prime}$ (either on an edge or a convex vertex of $\mathrm{P}^{\prime}$ ) and oriented in such a way that $\mathrm{P}^{\prime}$ completely belongs to the half-plane $\mathrm{H}_{l}$ monitored by the guard. In our problem every $180^{\circ}$-guard is replaced by a pair of towers $t_{1}$ and $t_{2}$ on the same edge of $\mathrm{P}^{\prime}$ in $\partial \mathrm{P}^{\prime} \cap \operatorname{kernel}\left(\mathrm{P}^{\prime}\right)$ and close to the $180^{\circ}$-guard. The orientation of $180^{\circ}$-guard is embedded into the tower coordinates via the parity trick. Notice that $L\left(t_{1}, t_{2}\right)$ is not always parallel to the line that supports $\mathrm{H}_{l}$. If the $180^{\circ}$-guard is positioned at a convex vertex $v$ of $\mathrm{P}^{\prime}$ then only one 
tower can be positioned at $v$. Another tower is placed on the edge adjacent to $v$ in $\partial \mathrm{P}^{\prime} \cap \operatorname{kernel}\left(\mathrm{P}^{\prime}\right)$. If $\operatorname{kernel}\left(\mathrm{P}^{\prime}\right)$ is a single point then we position our towers outside of $\mathrm{P}^{\prime}$ and close to $\operatorname{kernel}\left(\mathrm{P}^{\prime}\right)$, such that $L\left(t_{1}, t_{2}\right)$ is parallel to the line that supports $H_{l}$. If $L\left(t_{1}, t_{2}\right)^{+}$(respectively $\left.L\left(t_{1}, t_{2}\right)^{-}\right)$contains $\mathrm{H}_{l}$ then we position the towers at a distance which is a rational number whose numerator is even (respectively odd).

Algorithm 6 partitions $P$ and positions at most $\left\lfloor\frac{2 n}{3}\right\rfloor$ towers that can localize an agent anywhere in P. The localization algorithm (Algorithm 7) can be found in Chapter 18.

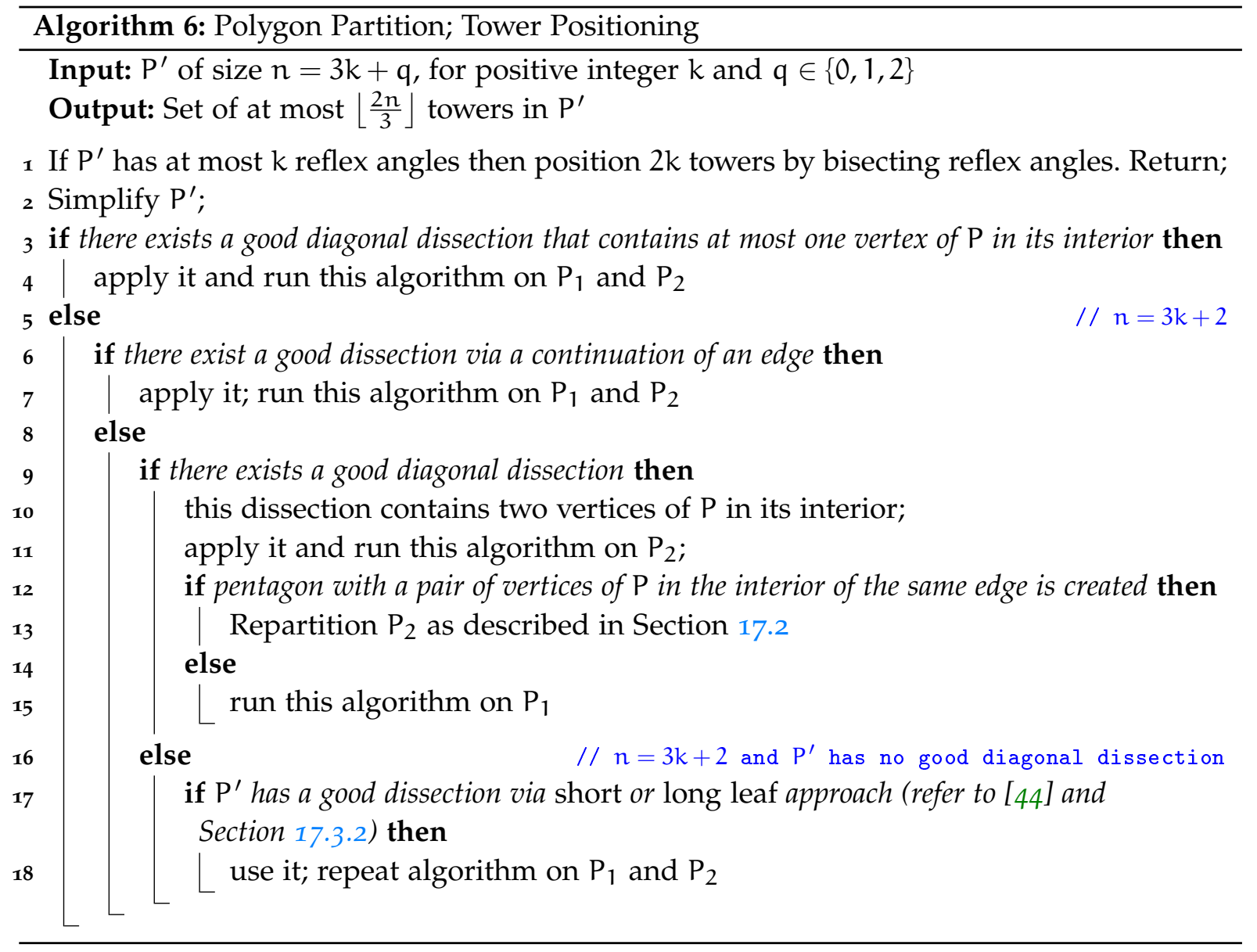

The running time of the algorithm is $\mathrm{O}\left(\mathrm{n}^{3}\right)$ because of the cases where repartitioning of already partitioned subpolygons is required.

\subsection{COUNTEREXAMPLE TO TÓTH'S CONJECTURE}

We show a counterexample to the conjecture given by Tóth in [44].

Conjecture: Any simple polygon of $n$ sides can be guarded by $\lfloor n / 3\rfloor 180^{\circ}$-guards that are located exclusively on the boundary of the polygon.

Figure 17.14 shows the smallest polygon $P$ with $n=8$ that can be guarded by 2 general $180^{\circ}$-guards but requires at least $3180^{\circ}$-guards that must reside on the boundary of $\mathrm{P}$. Observe that $\mathrm{P}$ is not a star-shaped polygon. Figure 17.14b shows a possible partition of $\mathrm{P}$ into two star-shaped polygons: $v_{1} v_{2} v_{3} v_{4} v_{5}$ and $v_{1} v_{5} v_{6} v_{7} v_{8}$. The polygon $v_{1} v_{2} v_{3} v_{4} v_{5}$ can be guarded by the $180^{\circ}$-guard $\mathrm{g}_{2}$ located on $\overline{v_{1} v_{5}}$ and oriented upwards (i.e. $g_{2}$ observes $\left.\mathrm{L}\left(v_{1}, v_{5}\right)^{+}\right)$. The second $180^{\circ}$-guard $\mathrm{g}_{1}$ is located on 
$\overline{v_{1} v_{8}}$ in kernel $\left(v_{1} v_{5} v_{6} v_{7} v_{8}\right)$. It is oriented to the right of $\mathrm{L}\left(v_{1}, v_{8}\right)$ (i.e. $g_{1}$ observes $\left.\mathrm{L}\left(v_{1}, v_{8}\right)^{-}\right)$and thus guards $v_{1} v_{5} v_{6} v_{7} v_{8}$. Consider Figure 17.14c. The visibility region, from where the complete interior of $v_{1} v_{2} v_{3} v_{4} v_{5}$ can be seen, is highlighted in magenta. We want to assign a single $180^{\circ}$-guard that can see both vertices $v_{2}$ and $v_{4}$ and be located on $\partial \mathrm{P}$. Notice that the intersection of this visibility region with $\partial \mathrm{P}$ contains a single point $v_{3}$. However, the angle of $\mathrm{P}$ at $v_{3}$ is reflex and the guards have a restricted $180^{\circ}$ field of vision. Thus it is impossible to guard $v_{1} v_{2} v_{3} v_{4} v_{5}$ with a single $180^{\circ}$-guard located on $\partial \mathrm{P}$. Notice that the visibility region of the vertex $v_{7}$ does not intersect with the visibility region of $v_{2}$ and the visibility region of $v_{4}$. Thus it requires an additional guard. It follows that $\mathrm{P}$ requires at least $3180^{\circ}$-guards located on $\partial \mathrm{P}$. Notice that $\lfloor n / 3\rfloor=\lfloor 8 / 3\rfloor=2$. This is a contradiction to the above conjecture.

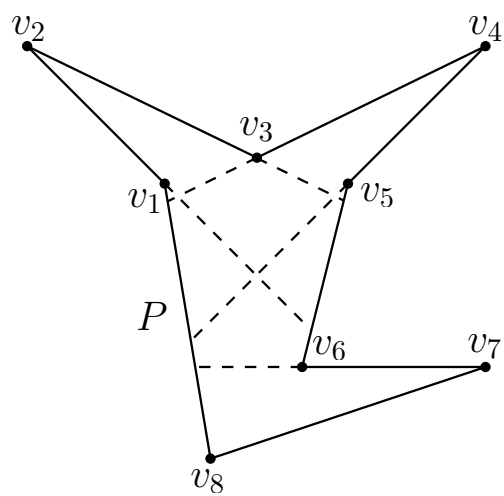

(a)

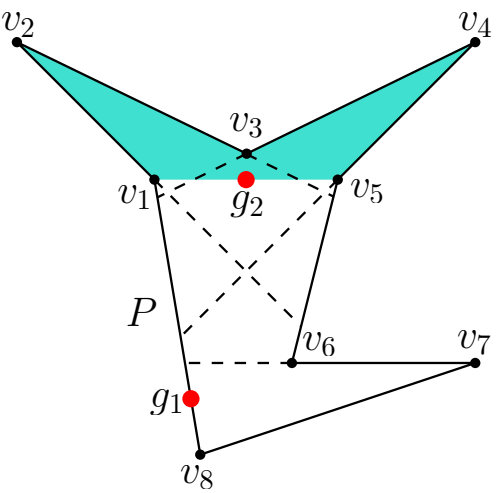

(b)

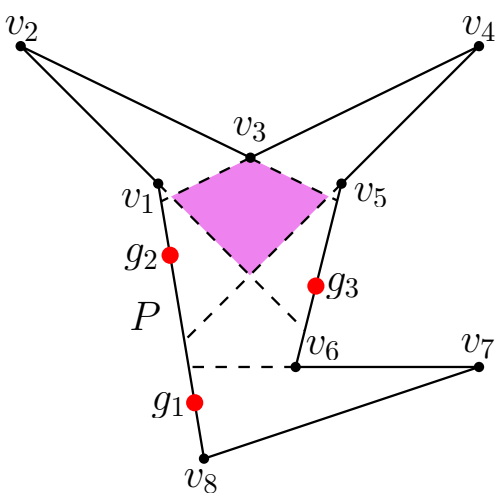

(c)

Figure 17.14: Smallest counterexample on $n=8$ vertices. Guards are highlighted in red. (b) $P$ can be guarded by 2 general $180^{\circ}$-guards(c) $P$ requires at least $3180^{\circ}$-guards that must reside on the boundary of $P$.

In general, consider the polygon shown in Fig. 17.15. It has $n=5 s+2$ vertices, where $s$ is the number of double-spikes. Each spike requires its own guard on the boundary of $\mathrm{P}$ (two guards per double-spike), resulting in $2 s$ boundary guards in total. This number is strictly bigger than $\lfloor n / 3\rfloor=\lfloor 5 s+2 / 3\rfloor$ for $s \geqslant 3$.

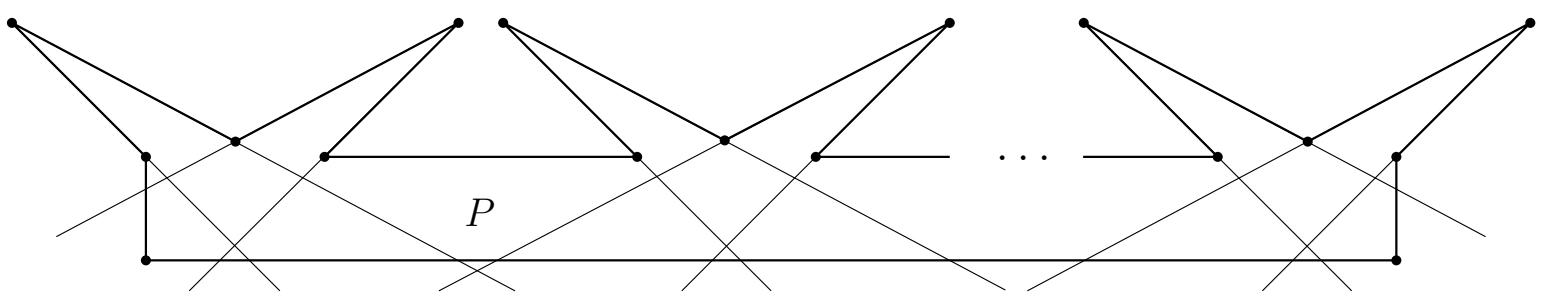

Figure 17.15: Counterexample to Tóth's conjecture. The polygon $\mathrm{P}$ is in general position. 
In Chapter 17 we showed how to position at most $\left\lfloor\frac{2 n}{3}\right\rfloor$ towers in a given polygon. We used a modification of Tóth's partition method that dissects a polygon into at most $\lfloor n / 3\rfloor$ star-shaped polygons each of which can be monitored by a pair of towers. In this section we show how we can localize an agent $p$ in the polygon. Our localization algorithm receives as input only the coordinates of the towers that can see $p$ together with their distances to $p$. In this sense, our algorithm uses the classical trilateration input. In addition, our algorithm knows that the parity trick was used to position the towers. Based on this information alone, and without any additional information about $P$, the agent can be localized. When only a pair of towers $t_{1}$ and $t_{2}$ can see the point $p \in P$ then the coordinates of the towers together with the distances $d\left(t_{1}, p\right)$ and $d\left(t_{2}, p\right)$ provide sufficient information to narrow the possible locations of $p$ down to two. Those two locations are reflections of each other over the line through $t_{1}$ and $t_{2}$. In this situation our localization algorithm uses the parity trick. It calculates the distance between the two towers and judging by the parity of the numerator of this rational number decides which of the two possible locations is the correct one. Refer to Algorithm 7.

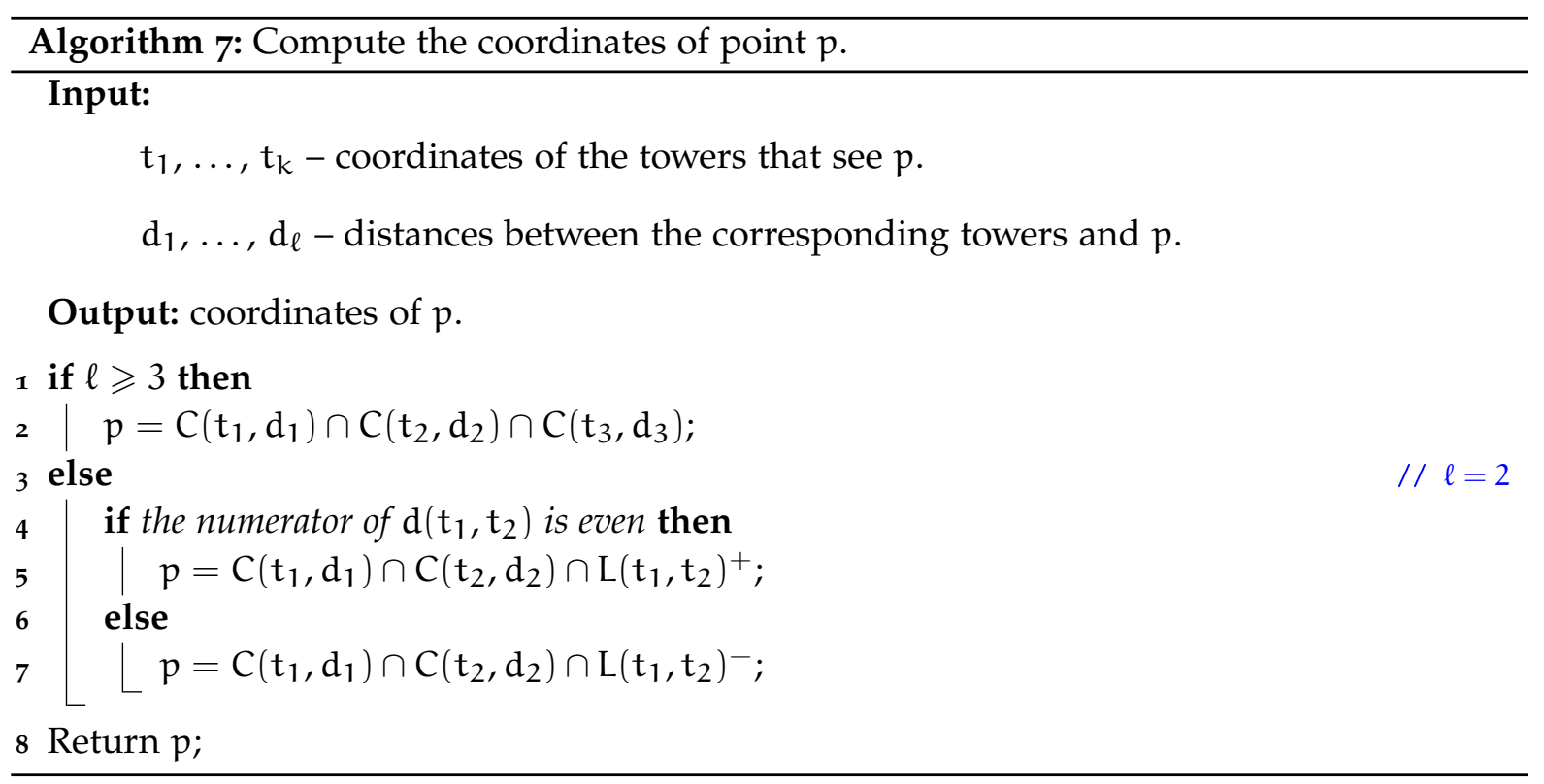


CONCLUDING REMARKS

19

We presented a tower-positioning algorithm that computes a set of size at most $[2 n / 3\rfloor$ towers, which improves the previous upper bound of $\lfloor 8 n / 9\rfloor[17]$. We strengthened the work [44] by lifting the assumption that the polygon partition produces polygons in general position. We reproved Tóth's result. We found and fixed several mistakes in [44].

We believe it is possible to avoid the repartition step (described in Section 17.2) and as a consequence bring the running time of Algorithm 6 to $\mathrm{O}\left(\mathrm{n}^{2}\right)$ instead of $\mathrm{O}\left(\mathrm{n}^{3}\right)$.

As a topic for future research we would like to show that determining an optimal set of towers for polygon trilateration is NP-hard. 
Part IV

OPTIMAL ART GALLERY LOCALIZATION IS NP-HARD 


\section{INTRODUCTION}

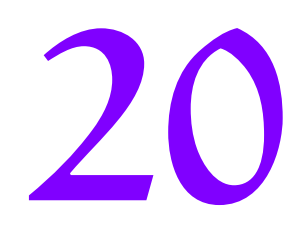

The art gallery problem was introduced by Victor Klee in 1973. He asked how many guards are sufficient to guard the interior of a simple polygon having $n$ vertices. It has been shown by Chvátal that $\lfloor n / 3\rfloor$ guards are always sufficient and sometimes necessary [13], and that such a set of guards can be computed easily [20]. However, such solutions are usually far from optimal in terms of minimizing the number of guards for a particular input polygon. Moreover, it was shown by Lee and Lin in [32] that determining an optimal set of guards is NP-hard, even for simple polygons. Refer also to the book "Art Gallery Theorems and Algorithms" by O'Rourke [37] that presents more detailed study of the topic.

In our research we combine the art gallery problem with trilateration. Trilateration is the process that determines absolute or relative locations of points by measurement of distances, using the geometry of the environment. Trilateration is not only interesting as a geometric problem, but it also has practical applications in surveying and navigation, including global positioning systems (GPS). GPS satellites carry very stable atomic clocks that are synchronized with one another and continuously transmit their current time and position. These signals are intercepted by a GPS receiver, which calculates how far away each satellite is based on how long it took for the messages to arrive. GPS receivers use trilateration to calculate the user's location, based on the information received from different GPS satellites.

By combining the art gallery problem with trilateration we address the problem of placing stationary broadcast towers in a simple polygon $\mathrm{P}$ in order for a receiving point $\mathrm{p}$ (let us call it an agent) to locate itself. Towers can be viewed as GPS satellites, while agents can be compared to GPS receivers. Towers are defined as points in a polygon $\mathrm{P}$ which can transmit their coordinates together with a time stamp to other points in their visibility region. In our context, trilateration is a process where the agent can determine its absolute coordinates from the messages the agent receives. The agent receives messages from all the towers that belong to its visibility region. Given a message from the tower $t$ the agent can determine its distance to $t$.

In [7] we showed how to position at most $\lfloor 2 n / 3\rfloor$ towers inside $P$ and gave a localization algorithm that receives as input only the coordinates of the towers that can see an agent $p$ together with their distances to $p$. We also showed that $\lfloor 2 n / 3\rfloor$ towers are sometimes necessary.

In this thesis we show that the problem of determining the minimum number of broadcast towers that can localize a point anywhere in P is NP-hard. Our solution is closely related to the NP-hardness of determining the minimum number of point guards for an n-edge simple polygon presented by Lee and Lin in [32]. To prove the NP-hardness of their problem, Lee and Lin show a reduction from Boolean Three Satisfiability (3SAT). We use a similar reduction to show that our problem is also NP-hard.

In Chapter 21 we give basic definitions and present some properties and observations. In Chapter 22 we present our main results of NP-hardness. Chapter 23 contains conclusions and possible ideas for future research. 


\section{PRELIMINARIES}

\section{1}

Let $\mathrm{P}$ be a simple polygon having a total of $n$ vertices on its boundary (denoted by $\partial \mathrm{P}$ ). Two points $u, v \in P$ are visible to each other if the segment $\overline{u v}$ is contained in $\mathrm{P}$; we also say that $u$ sees $v$. Note that $\partial \mathrm{P} \subseteq \mathrm{P}$ and that $\overline{u v}$ may touch $\partial \mathrm{P}$ in one or more points. For $u \in \mathrm{P}$ the visibility polygon of $u$ (denoted $V(u)$ ) is the set of all points $q \in P$ that are visible to $u$. Note that $V(u)$ is a star-shaped polygon contained in $\mathrm{P}$ and $\mathrm{u}$ belongs to its kernel (the set of points from which all of $\mathrm{V}(\mathrm{u})$ is visible).

Let $T$ be a set of points in P. Elements of $T$ are called towers. For any point $p \in P$ : for each $t \in T \cap V(p)$ the point $p$ receives the coordinates of $t$ and can compute the Euclidean distance between $t$ and $p$, denoted $d(t, p)$.

The set $T$ is called valid if for every point $p \in P$ its absolute location can be identified given the information it receives from every tower in $V(p)$. If $T$ is valid then we say that the polygon $P$ can be trilaterated with the set of towers $\mathrm{T}$. By the map of $\mathrm{P}$ we denote the complete information about $\mathrm{P}$ including the coordinates of all the vertices of $\mathrm{P}$ and the vertex adjacency list. We assume that our localization algorithm knows the map of $\mathrm{P}$ and the coordinates of all the towers in $\mathrm{T}$.

Let an agent $p$ be a point in the interior of $P$, whose location is unknown. $B y C(x, r)$ we denote the circle centered at $x$ with radius $r$. If only one tower $t$ can see $p$ then $p$ can be anywhere on $C(t, d) \cap V(t)$, which may not be sufficient to identify the location of $p$ (unless the agent and the tower are at the the same location). Refer to Fig. 21.1a. Notice that one must know the map of $P$ to calculate $V(t)$. Let $L(u, v)$ be the line through points $u$ and $v$. If a pair of towers $t_{1}$ and $t_{2}$ can see $p$ then the location of $p$ can be narrowed down to at most two points $C\left(t_{1}, d_{1}\right) \cap C\left(t_{2}, d_{2}\right) \cap V\left(t_{1}\right) \cap V\left(t_{2}\right)$ (which are reflections of each other along $L\left(t_{1}, t_{2}\right)$ ). Refer to Fig. 21.1b. However, if the map of $P$ is known (and thus we know $V\left(t_{1}\right)$ and $\left.V\left(t_{2}\right)\right)$ and if we place both towers on the same edge of $P$ in $\operatorname{kernel}(P) \cap \partial P$ then the intersection $C\left(t_{1}, d_{1}\right) \cap C\left(t_{2}, d_{2}\right) \cap V\left(t_{1}\right) \cap V\left(t_{2}\right)$ is a single point (highlighted in red on Fig. 21.1c). Notice, that in this case the kernel of $\mathrm{P}$ must not degenerate into a single point. Alternatively, if the map of $\mathrm{P}$ is unknown, we can place a triple of non-collinear towers in the kernel of star-shaped $\mathrm{P}$ (highlighted in cyan on Fig. 21.1d) to localize any point interior to P.

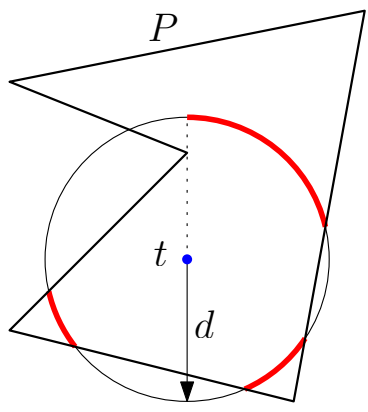

(a)

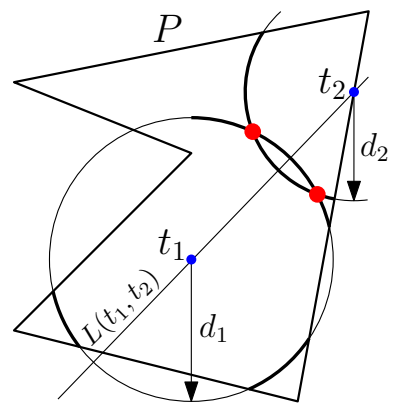

(b)

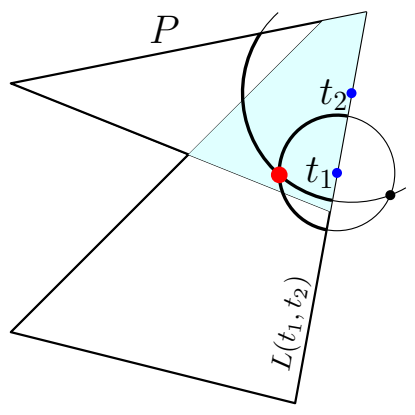

(c)

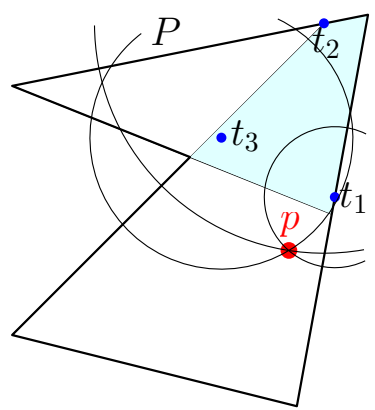

(d)

Figure 21.1: Trilateration example of a star-shaped polygon whose kernel does not degenerate into a single point. (a) The point $p$ can be anywhere on $C(t, d) \cap V(t)$ (highlighted in red). (b) Ambiguity along the line $\mathrm{L}\left(\mathrm{t}_{1}, \mathrm{t}_{2}\right) ; \mathrm{p}$ can be in one of the two possible locations (highlighted in red). (c) If the map of $P$ is known then the location of $p$ can be disambiguated and identified precisely. The kernel of $P$ is highlighted in cyan. (d) The location of $p$ can be identified precisely without any knowledge about $P$. 
To trilaterate a general simple polygon $\mathrm{P}$ we can partition it into star-shaped polygons $\mathrm{P}_{1}, \mathrm{P}_{2}, \ldots \mathrm{P}_{\mathrm{k}}$ such that: $P_{1} \cup P_{2} \cup \ldots \cup P_{k}=P$ and $\operatorname{kernel}\left(P_{i}\right)$, for every $1 \leqslant i \leqslant k$, does not degenerate into a single point; and then assign towers to each partition. Refer to [7] for a tower-positioning algorithm that computes $T$ of size at most $\lfloor 2 \mathrm{n} / 3\rfloor$.

The optimization problem we study is the following. Given a simple polygon $\mathrm{P}$, compute a set of towers $\mathrm{T}$ of minimum size that trilaterates $\mathrm{P}$. 
OPTIMAL ART GALLERY LOCALIZATION IS NP-HARD

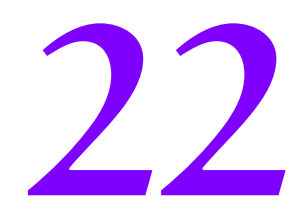

We want to show that determining a smalles set of towers for trilaterating a polygon is NP-hard. To do this, we need to give a reduction from a known NP-hard problem. Similarly to the approach used by Lee and Lin presented in [32], we reduce 3SAT to our problem.

Art Gallery Localization (AGL) Problem:

Instance: We are given a simple polygon $\mathrm{P}$ of size $\mathrm{n}$ and a positive integer $\mathrm{K}$.

Question: Does a valid set $T$ exist with $|\mathrm{T}| \leqslant \mathrm{K}$ ?

THEOREM 22.1. The art gallery localization problem is NP-hard.

To show NP-hardness we reduce the following NP-complete problem to AGL problem.

Boolean Three Satisfiability (3SAT) problem:

Instance: $\mathrm{A}$ set $\mathrm{U}=\left\{\mathrm{u}_{1}, \mathrm{u}_{2}, \ldots, \mathrm{u}_{\mathrm{n}}\right\}$ of Boolean variables and a collection $\mathrm{C}=\left\{\mathrm{c}_{1}, \mathrm{c}_{2}, \ldots, \mathrm{c}_{\mathrm{m}}\right\}$ of clauses over $U$ exist such that $c_{i} \in C$ is a disjunction (OR or $V$ ) of precisely three literals (where a literal is either a variable, or the negation of a variable).

Question: Does there exist a truth assignment to the $\mathrm{n}$ variables in $\mathrm{U}$ such that the conjunctive normal form $(\mathrm{CNF}) c_{1} \wedge c_{2} \wedge \ldots \wedge c_{m}$, evaluates to true?

We need to show that 3SAT can be transformed into AGL in polynomial time. In other words, the goal is to transform a given instance of 3SAT into a simple polygon with $\mathrm{O}\left((\mathrm{nm})^{\mathrm{d}}\right)$ vertices (for some constant $d$ ) that can be trilaterated with $K$ or fewer towers if and only if the 3SAT instance is satisfiable. Let the bound $K$ for the AGL problem be $8 m+2 n+2$.

We show how to construct a simple polygon in a step-by-step manner by describing the basic components from which the desired polygon is built. We describe a construction for literals, clauses (that contain several literals) and variables (where the consistency of true/false settings of the literals will be enforced). We want to construct a simple polygon such that no two different constructions can be completely visible to the same pair of towers.

\subsection{LITERAL PATTERN}

The construction $P_{l}$ for a literal $l$ is shown in Fig. 22.1. Notice that the vertices $a, d$ and $c$ are collinear.

The polygonal region $P_{l}$ is a star-shaped pentagon whose kernel is not a single point. Moreover, kernel $\left(\mathrm{P}_{l}\right) \cap \partial \mathrm{P}_{l}$ contains two distinct points that belong to the same edge of $\mathrm{P}_{l}$. Thus $\mathrm{P}_{l}$ can be trilaterated with as little as two towers since the map of the polygon is given. Let $x$ be a point where the extension of the edge $\overline{e d}$ intersects $\overline{c b}$. Refer to Fig. 22.1b. It follows, that both towers must either be on the line segment $\overline{x b}$ or on the edge $\overline{a b}$.

Notice that a point can see the complete interior of the triangle $\triangle a b c$ only if it is in the triangle. In other words, no tower from the outside of $\triangle a b c$ can see its complete interior.

If the literal $l$ is assigned true (respectively false) then the towers $t_{1}$ and $t_{2}$ are positioned at $\overline{a b}$ (respectively $\overline{x b}$ ). In particular, $t_{1}$ is positioned at vertex $a$ (respectively, at point $x$ ) and $t_{2}$ is positioned very close to $t_{1}$ on the corresponding line segment. Refer to Fig. 22.1C for the true assignment and to Fig. 22.Id for the false assignment. 


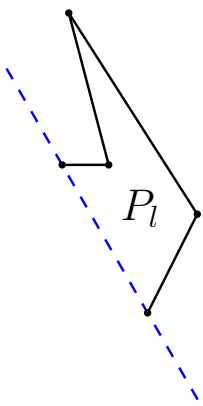

(a)

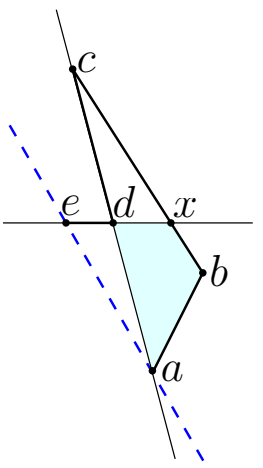

(b)

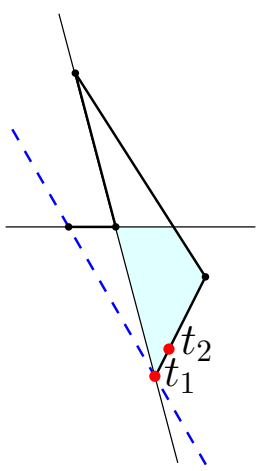

(c)

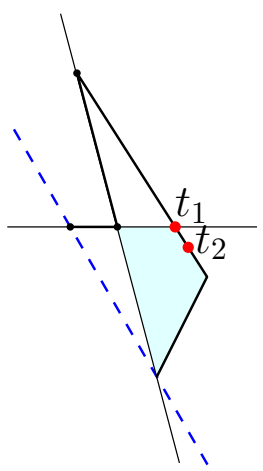

(d)

Figure 22.1: Construction $P_{l}$ of a literal $l$ (with the kernel highlighted in cyan). The blue dashed line indicates where this construction is attached to the main polygon. (b) The vertices $a, d$ and $c$ are collinear. Notice that $x$ is not a vertex of $P_{l}$. (c) The literal is assigned true or (d) false value.

\subsection{CLAUSE JUNCTION}

A construction $\mathrm{P}_{\mathrm{C}}$ for each clause $\mathrm{C}$ will contain four polygonal regions that represent literals. Three of them will correspond to the literals of the given clause. The role of the fourth polygonal region of a literal form will be explained later in this section. Note for now that it will always be assigned a value of true.

Consider the clause $C=l_{1} \vee l_{2} \vee l_{3}$, where $l_{1} \in\left\{u_{i}, \overline{u_{i}}\right\}, l_{2} \in\left\{u_{j}, \overline{u_{j}}\right\}$ and $l_{3} \in\left\{u_{k}, \overline{u_{k}}\right\}$ are literals, and $u_{i}, u_{j}$ and $u_{k}$ are variables, $u_{i}, u_{j}, u_{k} \in U$. The basic construction for the clause $C$ is shown in Fig. 22.2. It contains one subpolygon for each literal: $P_{l_{1}}=$ $\left\{a_{1}, b_{1}, c_{1}, d_{1}, e_{1}\right\}, P_{l_{2}}=\left\{a_{2}, b_{2}, c_{2}, d_{2}, e_{2}\right\}$ and $P_{l_{3}}=\left\{a_{3}, b_{3}, c_{3}, d_{3}, e_{3}\right\}$; and an additional subpolygon $P^{\prime}=\left\{a^{\prime}, b^{\prime}, c^{\prime}, d^{\prime}, e^{\prime}\right\}$ of a literal form. We show that every optimal solution for trilaterating $P_{C}$ must have one tower at the vertex $a^{\prime}$ and another anywhere on $\overline{a^{\prime} b^{\prime}}$ excluding $a^{\prime}$. The vertices $v_{3}, a^{\prime}, e^{\prime}, a_{3}, e_{3}, a_{2}, e_{2}, a_{1}, e_{1}$ and $v_{4}$ are collinear and belong to $\mathrm{L}\left(v_{3}, v_{4}\right)$.

We showed in Section 22.1 that each construction $\mathrm{P}_{l}$ requires at least two towers in its kernel. Notice that no tower can see the complete interior of $\mathrm{P}_{l}$ if it does not belong to $\mathrm{P}_{l}$. Since no two pentagons $\mathrm{P}_{l_{1}}, \mathrm{P}_{l_{2}}, \mathrm{P}_{\mathrm{l}_{3}}$ or $\mathrm{P}^{\prime}$ intersect, each of them requires at least two distinct towers. It

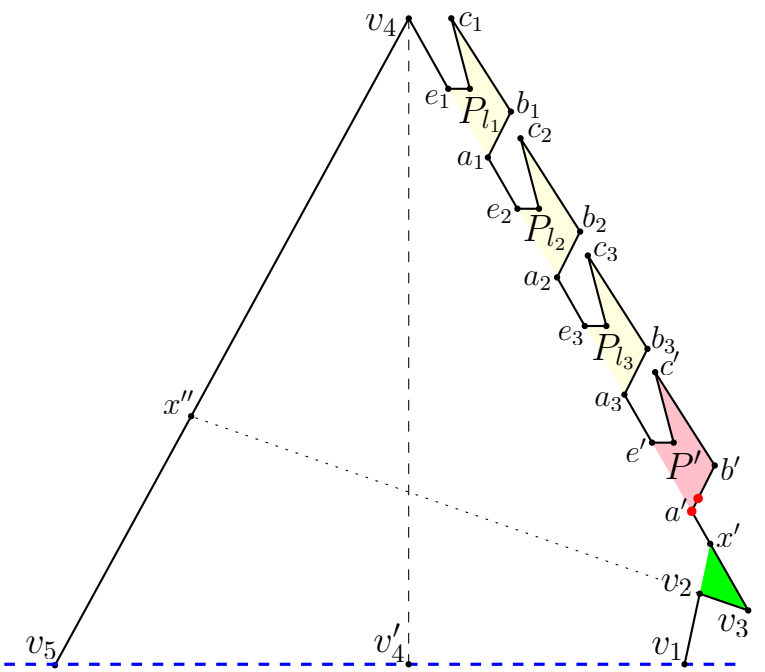

Figure 22.2: Construction $P_{C}$ of a clause $C$. The blue dashed line indicates where this construction is attached to the main polygon. Fixed towers are highlighted in red. follows, that we cannot trilaterate $\mathrm{P}_{\mathrm{C}}$ with less than 8 towers.

Let $x^{\prime}$ be a point where the extension of the edge $\overline{v_{1} v_{2}}$ intersects $\overline{v_{3} a^{\prime}}$. Assume that the solution for trilaterating $P_{C}$ contains a tower at $a^{\prime}$. The tower at $a^{\prime}$ can see the complete interior of $\Delta v_{2} v_{3} x^{\prime}$ (highlighted in green in Fig. 22.2). In order to localize an agent in $\Delta v_{2} v_{3} x^{\prime}$ we need at least one more tower on $\mathrm{L}\left(v_{3}, v_{4}\right) \cap \mathrm{P}_{\mathrm{C}}$. If one of the literals $l_{1}, l_{2}$ or $l_{3}$ is true, and thus there is a tower at $a_{1}, a_{2}$ or 
$a_{3}$, then we do not need any extra tower to locate an agent in $\Delta v_{2} v_{3} x^{\prime}$. However, if the solution for trilaterating $P_{C}$ does not have a tower at $a^{\prime}$ (as well as at $a_{1}, a_{2}$ and $a_{3}$ ) then there are must be at least two additional towers on the same edge of $\Delta v_{3} v_{4} x^{\prime \prime}$ (where $x^{\prime \prime}$ is an intersection point between the extension of the edge $\overline{v_{2} v_{3}}$ and $\overline{v_{4} v_{5}}$ ). It follows that the optimal solution for trilaterating $P_{c}$ must have a tower at $a^{\prime}$ and a tower at at least one of $a_{1}, a_{2}$ or $a_{3}$. Notice that the vertices $a_{1}, a_{2}, a_{3}$ and $a^{\prime}$ belong to the kernel of the pentagon $\left\{v_{1}, v_{2}, v_{3}, v_{4}, v_{5}\right\}$. Thus if at least one of the literals $l_{1}, l_{2}$ or $l_{3}$ is true then $P_{C}$ can be trilaterated with exactly 8 towers. However, if non of the three literals is true then we will need at least 9 towers for $P_{C}$ 's trilateration. Notice that $C$ has a truth value false if and only if the truth values of literals $l_{1}, l_{2}$ and $l_{3}$ are false. We just proved the following lemma:

LEMMA 22.2. A construction $\mathrm{P}_{\mathrm{C}}$ of a clause $\mathrm{C}$ can be trilaterated with 8 towers if and only if a truth value of $\mathrm{C}$ evaluates to true.

Notice that any tower that does not belong to $\mathrm{P}_{\mathrm{C}}$ cannot see all of $\Delta v_{2} v_{3} x^{\prime}$.

\subsection{VARIABLE PATTERN}

We need to create a construction that will force all truth assignments of literals of a particular variable to be consistent with one another. A variable pattern serves this purpose. You can see an example of the variable pattern $P_{i}$ for variable $u_{i}$ in Fig. 22.4 and at the bottom half of Fig. 22.3. One such pattern will exist per variable in the final construction.

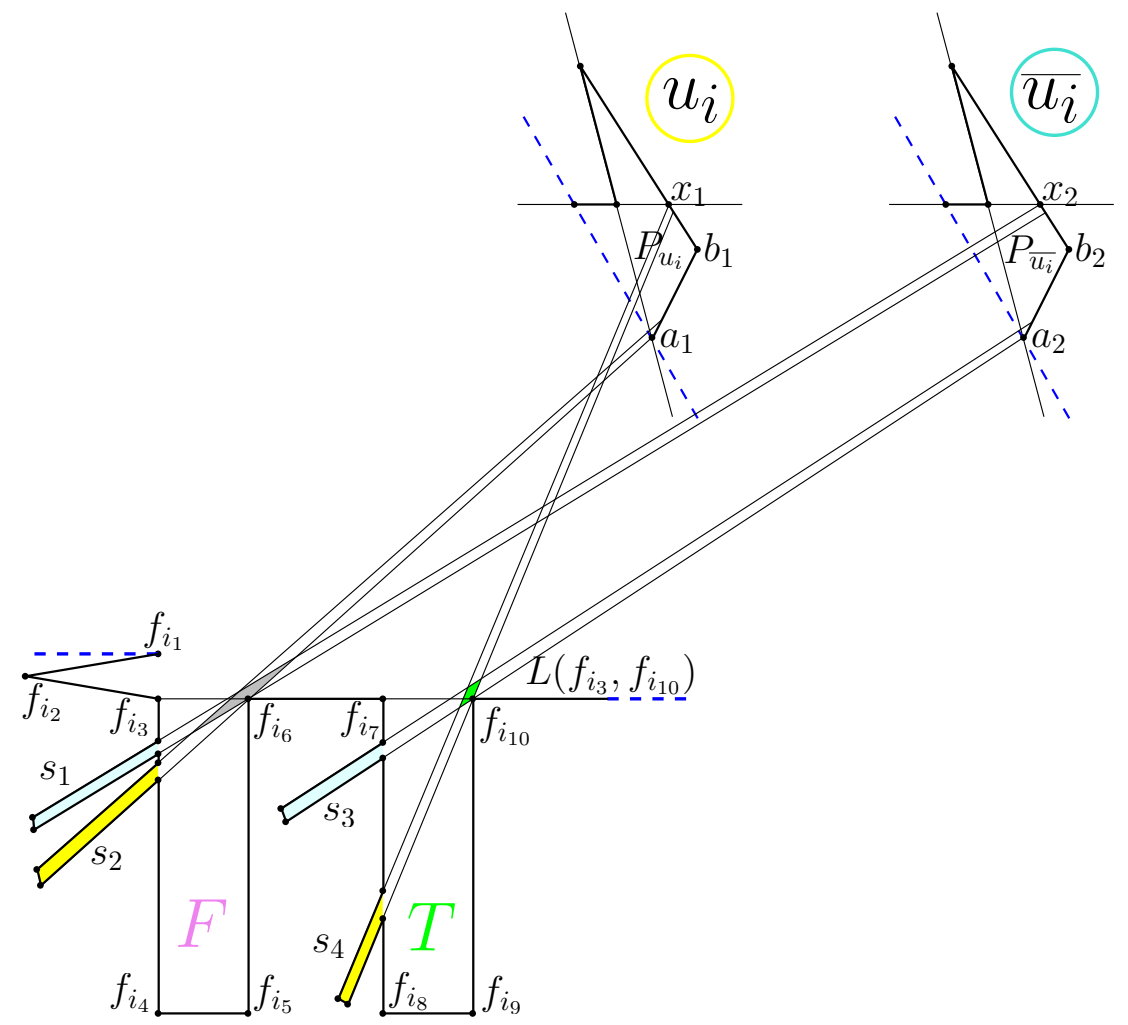

Figure 22.3: An example of a construction $P_{i}$ for the variable $u_{i}$. The blue dashed lines indicate where this construction is attached to the main polygon. Literal patterns $P_{u_{i}}$ and $P_{\overline{u_{i}}}$ may belong to the same clause or to different clauses.

Every variable pattern contains two wells. The leftmost well (marked with F in Fig. 22.3) will have one tower at the vertex $f_{i_{6}}$ and another in the close proximity to it (we will discuss later its specific 
location) if the given variable is assigned the truth value false. Let us call this an F-well. Similarly, if the variable is assigned the truth value true then the rightmost well (marked with T in Fig. 22.3) will have one tower at the vertex $f_{i_{10}}$ and another close to it. Let us call this a T-well. For simplicity we depicted wells here as vertical rectangles. However, their boundaries are not parallel to each other and in the complete construction are aligned with a pair of vertices whose construction will be defined in Section 22.4.

The wells contain spikes. The number of spikes each well contains in the final construction is equal to the number of times the corresponding variable or its negation appears in the given $3 \mathrm{CNF}$ formula. Suppose variable $u_{i}$ appears in clause $C$. The spikes in the F-well (respectively the T-well) for $u_{i}$ are aligned with the vertex $f_{i_{6}}$ (respectively, $f_{i_{10}}$ ) (and partially with its immediate neighbourhood).

If $C$, for example, contains the literal $l_{1}=u_{i}$ then the spike in the F-well (respectively, the Twell) that corresponds to $u_{i}$ in $C$ will also be aligned with $a_{1}$ (respectively, $x_{1}$ ) and its immediate neighbourhood on $\overline{a_{1} b_{1}}$ (respectively, $\overline{x_{1} b_{1}}$ ) of the literal pattern $P_{l_{1}}$ in clause junction $P_{C}$. Those spikes are $s_{2}$ and $s_{4}$ and they are highlighted in yellow in Fig. 22.3.

If $C^{\prime}$, for example, contains the literal $l_{2}=\overline{u_{i}}$ then the spike in the F-well (respectively, the Twell) that corresponds to $u_{i}$ in $C^{\prime}$ will also be aligned with $x_{2}$ (respectively, $a_{2}$ ) and its immediate neighbourhood on $\overline{x_{2} b_{2}}$ (respectively, $\overline{a_{2} b_{2}}$ ) of the literal pattern $P_{l_{2}}$ in clause junction $\mathrm{P}_{C^{\prime}}$. Those spikes are $s_{1}$ and $s_{3}$ and they are highlighted in cyan in Fig. 22.3.

Let us discuss ways in which a variable pattern can be trilaterated. Refer to Fig. 22.4. The visibility region of all the spikes in the F-well (respectively, the T-well) is highlighted in gray (respectively, green) and denoted $V\left(S_{i}^{F}\right)$ (respectively, $\left.\mathrm{V}\left(\mathrm{S}_{i}^{\top}\right)\right)$. It is always possible to construct a variable pattern in a way such that $V\left(S_{i}^{F}\right) \cap V\left(S_{i}^{\top}\right)=\emptyset$. This ensures that there does not exist a star-shaped polygon that contains a variable pattern as its subpolygon (connected via the blue dashed lines shown in Fig. 22.4). This means that the trilateration of a variable pattern cannot be done with less than 4 towers.

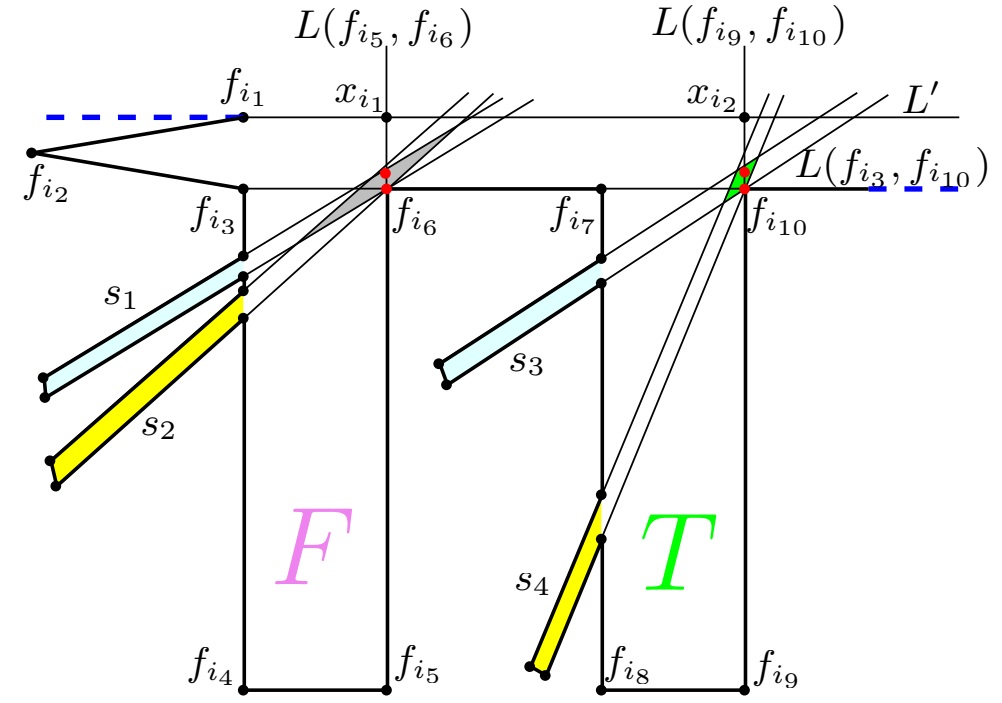

Figure 22.4: Example of a variable pattern $P_{i}$ for the variable $u_{i}$.

The vertices $f_{i_{3}}, f_{i_{6}}, f_{i_{7}}$ and $f_{i_{10}}$ are collinear and belong to $L\left(f_{i_{3}}, f_{i_{10}}\right)$. Let $L^{\prime}$ be the line parallel to $L\left(f_{i_{3}}, f_{i_{10}}\right)$ such that $f_{i_{1}} \in L^{\prime}$. Let $x_{i_{1}}$ (respectively, $\left.x_{i_{2}}\right)$ be an intersection point of $L^{\prime}$ and $L\left(f_{i_{5}}, f_{i_{6}}\right)$ (respectively, $L\left(f_{i_{9}}, f_{i_{10}}\right)$ ). The polygons $P_{i}^{F}=\left\{f_{i_{1}}, \ldots f_{i_{6}}, x_{i_{1}}\right\} \cup\{$ spikes of the F-well $\}$ and $P_{i}^{T}=\left\{f_{i_{1}}, f_{i_{2}}, f_{i_{3}}, f_{i_{7}} \ldots f_{i_{10}}, x_{i_{2}}\right\} \cup\{$ spikes of the T-well $\}$ are star-shaped by construction. Notice that $f_{i_{6}} \in \operatorname{kernel}\left(P_{i}^{\vec{F}}\right)$ and $f_{i_{10}} \in \operatorname{kernel}\left(P_{i}^{\top}\right)$. To trilaterate $P_{i}^{F}$ we position a pair of towers $t_{F}^{\prime}$ and $t_{F}^{\prime \prime}$ in kernel $\left(P_{i}^{F}\right)$. In particular, we position $t_{F}^{\prime}$ at $f_{i_{6}}$ and $t_{F}^{\prime \prime}$ in close proximity to $t_{F}^{\prime}$ in $L\left(f_{i_{5}}, f_{i_{6}}\right) \cap \operatorname{kernel}\left(P_{i}^{F}\right)$. In a similar way, to trilaterate $P_{i}^{\top}$ we position $t_{T}^{\prime}$ at $f_{i_{10}}$ and $t_{T}^{\prime \prime}$ in close proximity to $t_{T}^{\prime}$ in $L\left(f_{i_{9}}, f_{i_{10}}\right) \cap \operatorname{kernel}\left(P_{i}^{\top}\right)$. The locations of the four towers are highlighted in red in Fig. 22.4. Notice that both pairs of towers $t_{\mathrm{F}}^{\prime}, t_{\mathrm{F}}^{\prime \prime}$ and $t_{\mathrm{T}}^{\prime}, t_{\mathrm{T}}^{\prime \prime}$ can see $\triangle f_{i_{1}} f_{i_{2}} f_{i_{3}}$.

It is important to notice that despite that $t_{F}^{\prime}, t_{F}^{\prime \prime}$ are positioned on the boundary of $P_{i}^{F}$ and $t_{T}^{\prime}, t_{T}^{\prime \prime}$ are positioned on the boundary of $P_{i}^{\top}$, the towers $t_{F}^{\prime \prime}$ and $t_{T}^{\prime \prime}$ do not belong to the boundary of the main polygon. In Section 22.4, when we present the complete construction of the main polygon, we 
show that the ambiguities during the trilateration can be avoided because the map of $\mathrm{P}$ together with the coordinates of all the towers will be available to the agent. For now assume that the primary localization region of $t_{F}^{\prime}, t_{F}^{\prime \prime}$ is to the left of $L\left(t_{F}^{\prime}, t_{F}^{\prime \prime}\right)$ and that the primary localization region of $t_{T}^{\prime}, t_{T}^{\prime \prime}$ is to the left of $\mathrm{L}\left(\mathrm{t}_{\mathrm{T}}^{\prime}, \mathrm{t}_{\mathrm{T}}^{\prime \prime}\right)$.

We showed how to position 4 towers to trilaterate a variable pattern. However, only one of the two: $\mathrm{P}_{i}^{\mathrm{F}}$ or $\mathrm{P}_{i}^{\top}$ will require a pair of towers as described in the two previous paragraphs. No other tower will be needed to trilaterate the remainder of the variable pattern if all the literals for this variable are assigned truth values consistently. Consider an example of Fig. 22.3. Assume that $u_{i}$ is assigned the truth value true. Then we position towers in $P_{i}^{\top}$ only. We need to show that $P_{i}^{F}$ is trilaterated as well. All the literal patterns of the literals that equal to $u_{i}$ get a pair of towers in the "true" position, namely at and close to a-type vertices. Those towers can see inside the corresponding spikes in $\mathrm{P}_{i}^{F}$. All the literal patterns of the literals that equal to $\overline{u_{i}}$ get a pair of towers in the "false" position, namely at and close to $x$-type vertices. Those towers also can see inside the corresponding spikes in $P_{i}^{F}$. Thus, all the spikes in the F-well of $P_{i}^{F}$ are trilaterated. In addition, the towers of $P_{i}^{T}$ trilaterate $\left\{f_{i_{1}}, f_{i_{2}}, f_{i_{3}}, f_{i_{3}}, x_{i_{1}}\right\}$ - the subpolygon of $P_{i}^{F}$. The only subpolygon of $P_{i}^{F}$ that is not trilaterated is the quadrilateral $\left\{f_{i_{3}}, f_{i_{4}}, f_{i_{5}}, f_{i_{6}}\right\}$. Assume now that $u_{i}$ is assigned the truth value false. We position towers in $P_{i}^{F}$ only and need to show that $P_{i}^{\top}$ is trilaterated too. Similarly to the previous case it can be shown that all the spikes in the T-well of $P_{i}^{\top}$ are trilaterated. The towers of $P_{i}^{F}$ trilaterate $\left\{f_{i_{1}}, f_{i_{2}}, f_{i_{3}}, f_{i_{3}}, x_{i_{1}}\right\}$ - the subpolygon of $P_{i}^{\top}$. The only subpolygon of $P_{i}^{\top}$ that is not trilaterated is $\left\{x_{i_{1}}, f_{i_{6}}, f_{i_{7}}, f_{i_{8}}, f_{i_{9}}, x_{i_{2}}\right\}$. In the following section we show a position of a pair of towers that trilaterate all the subpolygons $\left\{f_{i_{3}}, f_{i_{4}}, f_{i_{5}}, f_{i_{6}}\right\}$ and $\left\{x_{i_{1}}, f_{i_{6}}, f_{i_{7}}, f_{i_{8}}, f_{i_{9}}, x_{i_{2}}\right\}$ for all $1 \leqslant i \leqslant n$.

Our discussion can be summarized in the following lemma.

LEMMA 22.3. All the spikes of the variable pattern for $u_{i}, 1 \leqslant i \leqslant n$ are trilaterated either by a pair of towers assigned to the variable pattern or by towers of the corresponding literal patterns in clause junctions. $\triangle \mathrm{f}_{i_{1}} \mathrm{f}_{\mathrm{i}_{2}} \mathrm{f}_{\mathrm{i}_{3}}$ is trilaterated by a pair of towers assigned to the variable pattern.

\subsection{COMPLETE CONSTRUCTION}

We put variable patterns and clause junctions together as shown in Fig. 22.5. This figure depicts an example of a complete polygon for a 3CNF formula $\left(u_{1} \vee \overline{u_{2}} \vee \overline{u_{3}}\right) \bigwedge\left(u_{1} \vee u_{2} \vee \overline{u_{3}}\right)$. This formula contains $n=3$ variables and $m=2$ clauses. Thus, the main polygon $P$ is comprised of 3 variable patterns and 2 clause junctions (each of which contains 4 literal patterns).

In Section 22.3 we described how the consistency of the assigned truth values is enforced among all the literals of a specific variable via spike construction. We also proposed to position only a pair of towers per variable pattern. However, it remained unanswered how to trilaterate all the wells that weren't assigned towers. Now we are able to address the issue. We align the boundaries of the wells for all variable patterns with a pair of vertices of P. Refer to Fig. 22.5. In particular, the left boundaries of both wells $\overline{f_{i_{3}} f_{i_{4}}}$ and $\overline{f_{i_{7}} f_{i_{8}}}$ for each variable pattern are aligned with the vertex $w_{2}$ (i.e. $w_{2} \in$ $L\left(f_{i_{3}}, f_{i_{4}}\right)$ and $w_{2} \in L\left(f_{i_{7}}, f_{i_{8}}\right)$ for $\left.1 \leqslant i \leqslant n\right)$. Similarly, the right boundaries of both wells $\overline{f_{i_{5}} f_{i_{6}}}$ and $\overline{f_{i_{9}} f_{i_{10}}}$ for each variable pattern are aligned with the vertex $w_{1}$. Notice that the pair of vertices $w_{1}, w_{2}$ can see the interior of all the wells, and in particular, all the polygons $\left\{f_{i_{3}}, f_{i_{4}}, f_{i_{5}}, f_{i_{6}}\right\}$ and $\left\{x_{i_{1}}, f_{i_{6}}, f_{i_{7}}, f_{i_{8}}, f_{i_{9}}, x_{i_{2}}\right\}$ for all $1 \leqslant i \leqslant n$. Let $w$ be an intersection point of $L\left(f_{1_{5}}, f_{1_{6}}\right)$ and $L\left(f_{n_{7}}, f_{n_{8}}\right)$. Notice that $\triangle w_{w_{1}} w_{3}$ is the kernel of the polygon $\left\{w_{1}, w_{2}, w_{3}, w_{4}, w_{5}\right\} \cup\{$ all the wells $\}$. In addition, $\triangle w_{w_{1}} w_{3} \cap \partial \mathrm{P}=\overline{w_{1} w_{2}}$. We position a pair of towers: one at $w_{1}$ and another at $w_{2}$. Now all the wells and the polygon $\left\{w_{1}, w_{2}, w_{3}, w_{4}, w_{5}\right\}$ are trilaterated with a pair of towers.

Recall, that in Section 22.3 we positioned the towers $t_{F}^{\prime \prime}$ and $t_{T}^{\prime \prime}$ not on the boundary of $P$. We show that the ambiguities can be avoided because the map of $\mathrm{P}$ together with the coordinates of all the towers is available to the agent. Assume for example that an agent reserved messages from only two towers $t_{F}^{\prime}$ and $t_{F}^{\prime \prime}$ of variable pattern for $u_{i}$ (refer to Fig. 22.4, 22.5). Thus, the agent can 


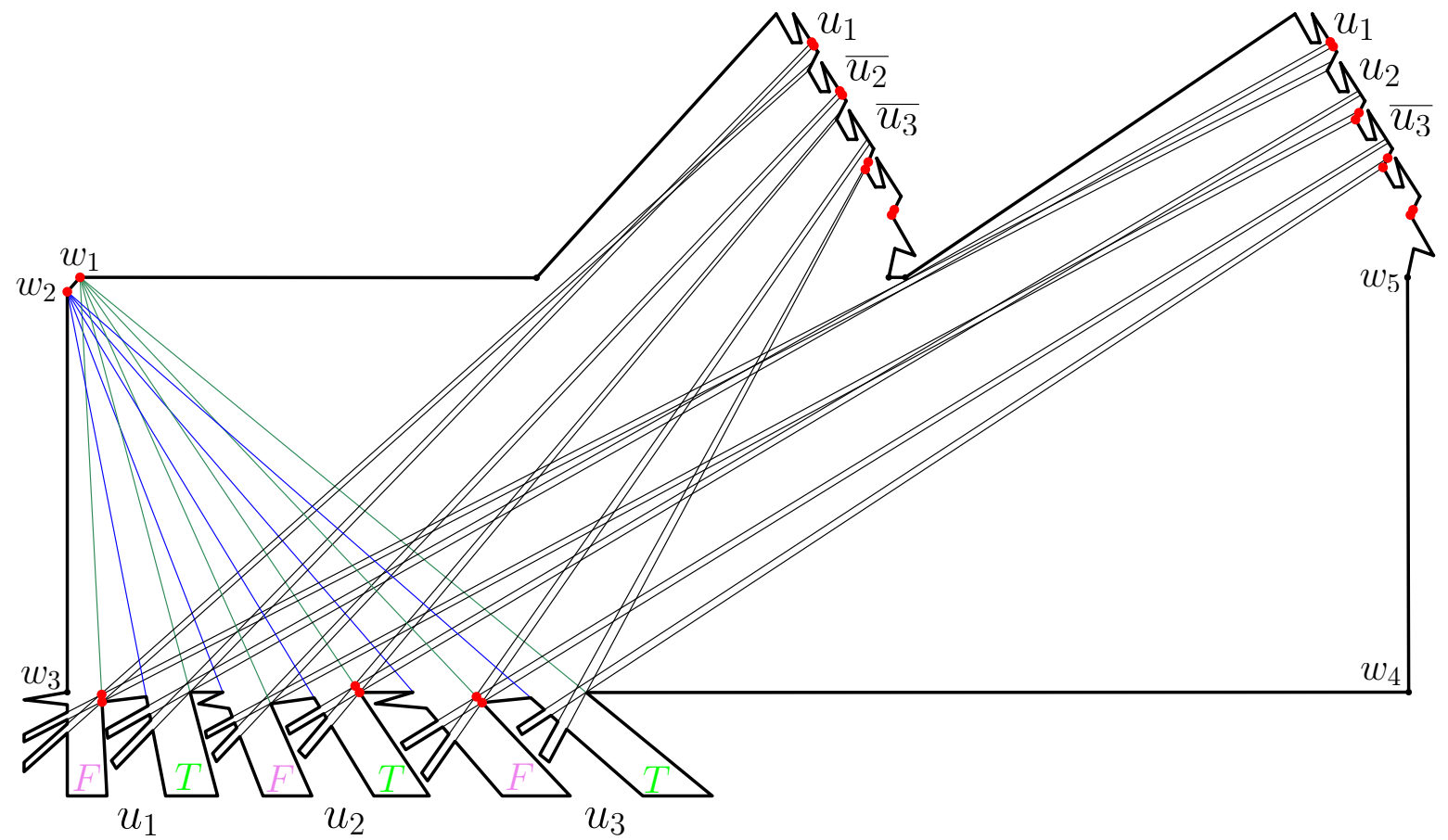

Figure 22.5: The complete polygon $P$ for $\left(u_{1} \vee \overline{u_{2}} \vee \overline{u_{3}}\right) \bigwedge\left(u_{1} \vee u_{2} \vee \overline{u_{3}}\right)$ with an example of 8m + $2 n+2=24$ towers (shown in red) which is minimum. The tower positions correspond to the following truth assignments: $\mathrm{u}_{1}, \mathrm{u}_{3}$ are false; $\mathrm{u}_{2}$ is true.

be in one of the two locations that are reflections of each other along $L\left(t_{F}^{\prime}, t_{F}^{\prime \prime}\right)$; assuming also that both locations belong to $\mathrm{V}\left(\mathrm{t}_{\mathrm{F}}^{\prime}\right) \cap \mathrm{V}\left(\mathrm{t}_{\mathrm{F}}^{\prime \prime}\right)$ (if not, - there is no ambiguity). Observe that if the agent is situated in the clause junction $C$ then it is seen by at least one tower of the 4 literal patterns of $C$. Thus the agent cannot be in any clause junction, because it is seen by $t_{F}^{\prime}$ and $t_{F}^{\prime \prime}$ only. Notice that $\left(\left(\mathrm{V}\left(\mathrm{t}_{\mathrm{F}}^{\prime}\right) \cap \mathrm{V}\left(\mathrm{t}_{\mathrm{F}}^{\prime \prime}\right)\right) \backslash\left(\mathrm{V}\left(w_{1}\right) \cap \mathrm{V}\left(w_{2}\right)\right)\right) \backslash\{$ all the clause junctions $\}$ consists only of the spikes of the F-well of variable pattern for $u_{i}$ and $\triangle f_{i_{1}} f_{i_{2}} f_{i_{3}}$. The agent has the map of $P$ and knows the positions of all the towers. Therefore, the agent can calculate all the necessary visibility regions. Notice also that the spikes of the F-well for $u_{i}$ and $\triangle f_{i_{1}} f_{i_{2}} f_{i_{3}}$ belong to one side of $L\left(t_{F}^{\prime}, t_{F}^{\prime \prime}\right)$. Thus, if the agent is seen by $t_{F}^{\prime}$ and $t_{F}^{\prime \prime}$ only then it has a unique location at $\left(\left(V\left(t_{F}^{\prime}\right) \cap V\left(t_{F}^{\prime \prime}\right)\right) \backslash\left(V\left(w_{1}\right) \cap V\left(w_{2}\right)\right)\right) \backslash\{$ all the clause junctions\}. Similarly, if the agent is seen by $t_{T}^{\prime}$ and $t_{T}^{\prime \prime}$ only then it has a unique location at $\left(\left(\mathrm{V}\left(\mathrm{t}_{\mathrm{T}}^{\prime}\right) \cap \mathrm{V}\left(\mathrm{t}_{\mathrm{T}}^{\prime \prime}\right)\right) \backslash\left(\mathrm{V}\left(w_{1}\right) \cap \mathrm{V}\left(w_{2}\right)\right)\right) \backslash\{$ all the clause junctions $\}$.

LeMmA 22.4. At least $\mathrm{K}=8 \mathrm{~m}+2 \mathrm{n}+2$ towers are required to trilaterate $\mathrm{P}$.

Proof. We showed in Section 22.2 that a clause junction can be trilaterated with at least 8 towers. In Section 22.3 we discussed that each variable pattern requires at least 2 towers. In this section we showed that an additional 2 towers are necessary to trilaterate $\left\{w_{1}, w_{2}, w_{3}, w_{4}, w_{5}\right\} \cup\{$ all the wells $\}$. Since $P$ is the union of $m$ clause junctions, $n$ variable patterns and the polygon $\left\{w_{1}, w_{2}, w_{3}, w_{4}, w_{5}\right\}$, then its trilateration requires at least $K=8 m+2 n+2$ towers.

LEMMA 22.5. The minimum number of towers required to trilaterate the main polygon is $\mathrm{K}=8 \mathrm{~m}+2 \mathrm{n}+2$ if and only if the given $3 \mathrm{CNF}$ formula is satisfiable.

Proof. $(\leftarrow)$ Assume that the given $3 \mathrm{CNF}$ formula is satisfiable. Then a truth assignment to the variables exists such that each of the clauses $C_{j}, 1 \leqslant j \leqslant m$ has a truth value true. By Lemma 22.2 every clause junction can be trilaterated with 8 towers. Lemma 22.3 implies that 2 towers per variable pattern is sufficient to trilaterate all the spikes (that where not trilaterated by the towers in clause junctions) and 
$\triangle f_{i_{1}} f_{i_{2}} f_{i_{3}}$ for $1 \leqslant i \leqslant n$. We showed in this section that an additional 2 towers trilaterate the remaining uncovered subpolygons of $P$ and resolve ambiguities. Thus $P$ can be trilaterated with $K=8 m+2 n+2$ towers, which by Lemma 22.4 is the minimum number.

$(\rightarrow)$ Assume that the given $3 \mathrm{CNF}$ formula is not satisfiable. It follows that there exists at least one clause $C_{j}$ that has a truth value false under any truth assignment. By Lemma 22.2 the clause junction $\mathrm{P}_{\mathrm{C}_{j}}$ cannot be trilaterated with 8 towers. Similar to the proof of Lemma 22.4 $\mathrm{P}$ requires at least $K=8 m+2 n+3$ towers for trilateration.

\subsection{CONSTRUCTION TAKES POLYNOMIAL TIME.}

To complete the proof of our main result - Theorem 22.1, we need to show that the reduction takes polynomial time. We have to demonstrate that the number of vertices of $P$ is polynomial in $n$ and $m$ and that the number of bits in the binary representation of the coordinates of those vertices is bounded by a polynomial in $n$ and $m$. During the reduction we create a simple polygon $P$ of size $49 m+10 n+3$. Every clause junction consists of 25 vertices. Every literal creates two spikes, thus the total number of vertices in $P$ occupied by spikes is $24 \mathrm{~m}$. Every variable pattern (without spikes) consists of 10 vertices. The final construction of $P$ includes 3 more vertices for $w_{1}, w_{2}$ and $w_{4}$. Thus, the total number of vertices of $P$ is $25 m+24 m+10 n+3=49 m+10 n+3$, which is polynomial in $n$ and $m$. Notice that $n \leqslant 3 m$ thus instead of saying "polynomial in $n$ and $m$ " we can just use "polynomial in $m$ ".

Consider a construction $\mathrm{P}_{\mathrm{C}}$ of a clause $\mathrm{C}$ (refer to Fig. 22.2). Let $v_{4}^{\prime}$ be an orthogonal projection of $v_{4}$ onto $\mathrm{L}\left(v_{5}, v_{1}\right)$. We would like to fix the part of $\mathrm{P}_{\mathrm{C}}$ to the right of $\mathrm{L}\left(v_{4}, v_{4}^{\prime}\right)$ and keep it identical among all the clauses of the given $3 \mathrm{CNF}$ formula. Assume that $v_{4}^{\prime}$ is the origin of the coordinate system. We can change the positions of all the vertices of $\mathrm{P}_{\mathrm{C}}$ to the right of $\mathrm{L}\left(v_{4}, v_{4}^{\prime}\right)$ (keeping collinearities and main features intact) such that the binary representation of their coordinates is polynomial in $\mathrm{m}$. The relative position of the vertex $v_{5}$ to other vertices of $C$ will differ from clause to clause (refer to Fig. 22.6).

In the complete construction of $\mathrm{P}$ we position $w_{3}$ at the origin of the coordinate system, i.e. the coordinates of $w_{3}$ are $(0,0)$. When we add all the variable patterns $P_{u_{i}}, 1 \leqslant i \leqslant n$ to $P$ we can position every following variable pattern $P_{u_{i+1}}$ slightly below (by $y$-coordinate) $P_{u_{i}}$, otherwise the towers in $P_{u_{i+1}}$ could see $\triangle f_{i_{1}} f_{i_{2}} f_{i_{3}}$. Alternatively, we can rotate the line $L\left(f_{i_{3}}, f_{i_{10}}\right)$ around $f_{i_{3}}$ to contain $x_{i_{2}}$ (refer to Fig. 22.4) and extend the boundaries of the wells such that $f_{i_{10}}=x_{i_{2}}$ and $f_{i_{6}}, f_{i_{7}} \in L\left(f_{i_{3}}, f_{i_{10}}\right)$. We will use the latter approach. Now we can set the $y$-coordinate of the vertices $f_{1_{1}}, f_{1_{10}}, f_{2_{1}}, f_{2_{10}}, \ldots$ , $f_{n_{1}}, f_{n_{10}}$ to 0 (note that $w_{3}=f_{1_{1}}$ ).

Let $k_{0}, k_{1}, k_{2}, k_{3}, k_{4}, k_{5}, k_{6}$ be positive numbers of polynomial size in $m$ sorted in the increasing order such that $k_{6}<2 k_{5}$. We set the $x$-coordinate of $w_{1}$ to be $k_{1}$ and its $y$-coordinate to be $k_{5}$. In other words, $w_{1}=\left(k_{1}, k_{5}\right)$. The $y$-coordinate of $w_{2}$ is set to $k_{4}$, i.e. $w_{2}=\left(0, k_{4}\right)$. We set the distance between the vertices $f_{i_{1}}$ and $f_{i_{10}}$ for $1 \leqslant i \leqslant n$ to be $3 k_{2}$ and the distance between $f_{i_{10}}$ and $f_{i+1}$ for $1 \leqslant i<n$ to be $k_{2}$. Refer to Figures 22.4 and 22.6. Recall that the $y$-coordinate of $f_{i_{1}}, f_{i_{10}}$ for $1 \leqslant i \leqslant n$ is 0 . We just defined the vertices $f_{i_{1}}=\left(4 k_{2}(i-1), 0\right)$ and $f_{i_{10}}=\left(3 k_{2}+4 k_{2}(i-1), 0\right)$ for $1 \leqslant i \leqslant n$. Notice that all the coordinates defined so far are polynomial in $\mathrm{m}$.

The $y$-coordinate of $f_{i_{3}}$ for $1 \leqslant i \leqslant n$ is set to $-k_{0}$. To determine its $x$-coordinate observe that $f_{i_{3}}$, $1 \leqslant i \leqslant n$ is an intersection point between $L\left(w_{2}, f_{i_{1}}\right)$ and the horizontal line through $\left(0,-k_{0}\right)$. To proceed further recall the following. 


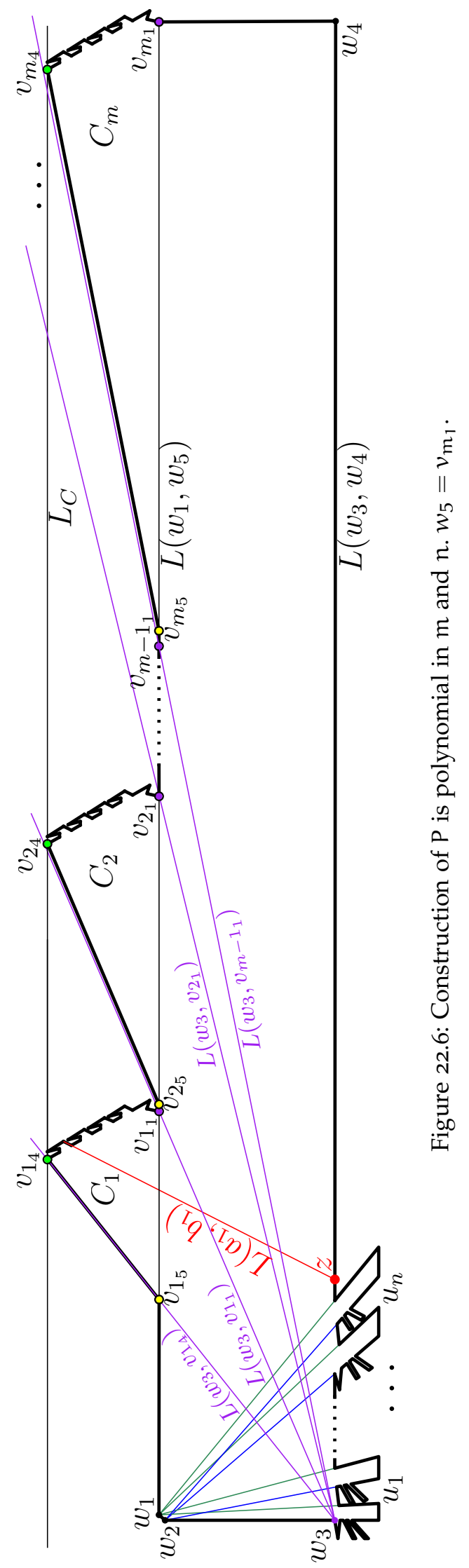


The intersection point of two lines $L_{1}$ (defined by two distinct points $\left(x_{1}, y_{1}\right)$ and $\left(x_{2}, y_{2}\right)$ ) and $L_{2}$ (defined by two distinct points $\left(x_{3}, y_{3}\right)$ and $\left.\left(x_{4}, y_{4}\right)\right)$ can be written out as

$$
\begin{aligned}
(x, y)= & \left(\frac{\left(x_{1} y_{2}-y_{1} x_{2}\right)\left(x_{3}-x_{4}\right)-\left(x_{1}-x_{2}\right)\left(x_{3} y_{4}-y_{3} x_{4}\right)}{\left(x_{1}-x_{2}\right)\left(y_{3}-y_{4}\right)-\left(y_{1}-y_{2}\right)\left(x_{3}-x_{4}\right)},\right. \\
& \left.\frac{\left(x_{1} y_{2}-y_{1} x_{2}\right)\left(y_{3}-y_{4}\right)-\left(y_{1}-y_{2}\right)\left(x_{3} y_{4}-y_{3} x_{4}\right)}{\left(x_{1}-x_{2}\right)\left(y_{3}-y_{4}\right)-\left(y_{1}-y_{2}\right)\left(x_{3}-x_{4}\right)}\right) .
\end{aligned}
$$

This computation yields a fraction whose numerator is a polynomial of degree three in the input coordinates and whose denominator is a polynomial of degree two in the input coordinates. That is, if the input coordinates are $b$-bit numbers then the output coordinates need at most $5 b$ bits to be represented. The proof of this can be found in lecture notes in Computational Geometry by M. Hoffmann [26].

Thus, the $x$-coordinate of $f_{i_{3}}, 1 \leqslant i \leqslant n$ can be determined via the above computation. Moreover, the number of bits required for its $x$-coordinate is at most 5 times the number of bits used for $f_{i_{1}}$ (assuming $f_{i_{1}}$ had the most bits in its representation among $w_{3}, f_{i_{1}},\left(0,-k_{0}\right)$ and $\left(1,-k_{0}\right)$ ), and it is still polynomial in $\mathrm{m}$.

In a similar way we find the coordinates of other vertices that define wells. The vertex $f_{i_{6}}$ for $1 \leqslant i \leqslant n$ is an intersection of $L\left(w_{1},\left(k_{2}+4 k_{2}(i-1), 0\right)\right)$ and $L\left(f_{i_{3}}, f_{i_{10}}\right)$; and the vertex $f_{i_{7}}$ for $1 \leqslant i \leqslant n$ is an intersection of $L\left(w_{2},\left(2 k_{2}+4 k_{2}(i-1), 0\right)\right)$ and $L\left(f_{i_{3}}, f_{i_{10}}\right)$. We set the $y$-coordinate of all the vertices at the bottom of every well to $-k_{3}$. Thus the $x$-coordinate of $f_{i_{4}}$ (respectively $f_{i_{5}}, f_{i_{8}}$ and $\left.f_{i_{g}}\right)$ for $1 \leqslant i \leqslant n$ is equal to the $x$-coordinate of the intersection point between the horizontal line through $\left(0,-k_{3}\right)$ and $L\left(w_{2}, f_{i_{1}}\right)$ (respectively $L\left(w_{1},\left(k_{2}+4 k_{2}(i-1), 0\right)\right), L\left(w_{2},\left(2 k_{2}+4 k_{2}(i-1), 0\right)\right)$ and $\left.L\left(w_{1}, f_{i_{10}}\right)\right)$. Notice that the number of bits required to represent the coordinates of each of the discussed vertices is polynomial in $m$ and is at most 5 times the number of bits required to represent $f_{i_{1}}$ or $f_{i_{10}}$. The vertex $f_{i_{2}}, 1 \leqslant i \leqslant n$ does not require precise construction; there is plenty of room to choose polynomial coordinates for it.

We proceed now with the construction of the clause junctions and after that we will return to discuss the spike formation inside the variable patterns.

Consider the first clause $C_{1}$ in the given $3 C N F$ formula. Let $P_{C_{1}}$ be its clause junction. Let $z$ be an intersection point of the two lines $\mathrm{L}\left(\mathrm{a}_{1}, \mathrm{~b}_{1}\right)$ and $\mathrm{L}\left(w_{3}, w_{4}\right)$ (refer to Figures 22.2 and 22.6). We position $\mathrm{P}_{\mathrm{C}_{1}}$ in $\mathrm{P}$ in such a way that the $\mathrm{x}$-coordinate of $z$ is not smaller than the $x$-coordinate of $\mathrm{f}_{\mathrm{n}_{10}}$. Otherwise, we cannot guarantee that the towers of every literal pattern can see the vertices $f_{i_{1}}, f_{i_{10}}$ for $1 \leqslant i \leqslant n$. By construction the angle that $\mathrm{L}\left(\mathrm{a}_{1}, \mathrm{~b}_{1}\right)$ creates with the positive direction of $X$-axis is bigger than $\pi / 3$. Thus if we set the $x$-coordinate of $v_{1_{4}}$ (where $v_{1_{4}}$ is the vertex $v_{4}$ of the first clause $C_{1}$ ) to be twice bigger than the $x$-coordinate of $f_{n_{10}}$, then $z$ is guaranteed to be to the right of $f_{n_{10}}$. Hence, we set $v_{1_{4}}=\left(2 k_{2}(4 n-1), k_{6}\right)$ (note that $\left.f_{n_{10}}=\left(3 k_{2}+4 k_{2}(n-1), 0\right)=\left(k_{2}(4 n-1), 0\right)\right)$. Recall that in the beginning of this section we fixed the part of $\mathrm{P}_{\mathrm{C}_{j}}$ to the right of $L\left(v_{j_{4}}, v_{j_{4}}^{\prime}\right)$ for $1 \leqslant j \leqslant m$ and assigned polynomial coordinates to all the vertices of $\mathrm{P}_{\mathrm{C}_{j}}$ except for $v_{j_{5}}$ (assuming that $v_{j_{4}}$ is the origin). To add $P_{C_{1}}$ to $P$ we just translate all the vertices of $P_{C_{1}}$ (except for $\left.v_{1_{5}}\right)$ by vector $\left(2 k_{2}(4 n-1), k_{5}\right)$. The $y$ coordinate of $v_{1_{5}}$ is $k_{5}$. Its $x$-coordinate should be small enough for the towers inside the first literal of $C_{1}$ to see $f_{1_{3}}$. Since $k_{5}>k_{6} / 2$ there are plenty of polynomial choices for $v_{1_{5}}$. Notice that the number of bits in binary representation of the coordinates of all the vertices of $\mathrm{P}_{\mathrm{C}_{1}}$ is bounded by polynomial in $\mathrm{m}$.

To add the clause junction $P_{C_{2}}$ of the second clause $C_{2}$ to $P$ we consider the line $L\left(w_{3}, v_{1_{1}}\right)$. Refer to Fig. 22.6. Let $h$ be the intersection point between $L\left(w_{3}, v_{1_{1}}\right)$ and the horizontal line through $\left(0, k_{6}\right)$. The $y$-coordinate of $v_{2_{4}}$ is set to $k_{6}$, and its $x$-coordinate should be to the right of $h$. Since $k_{5}$ is bigger than $k_{6}-k_{5}$, the length of the line segment $\overline{w_{3} v_{1_{1}}}$ is bigger then the length of $\overline{v_{1_{1}} h}$. Hence, if we set 
the $x$-coordinate of $v_{2_{4}}$ to be twice bigger then the $x$-coordinate of $v_{1_{1}}$ then $v_{2_{4}}$ is guaranteed to be to the right of $h$. Moreover, the number of bits of the $x$-coordinate of $v_{2_{4}}$ will be larger then the number of bits of the $x$-coordinate of $v_{1}$ by at most 1 . All other vertices of $P_{C_{2}}$ can be added similarly to the vertices of $P_{C_{1}}$ (via the translation by the vector $\left(2\left\{x\right.\right.$-coordinate of $\left.\left.v_{1_{1}}\right\}, k_{5}\right)$ ). The vertex $v_{2_{4}}$ can be positioned close to $v_{1_{1}}$ or even at $v_{1_{1}}$.

We keep on adding clause junctions $P_{C_{j}}$ for $1 \leqslant j \leqslant m$ to $P$ in a similar manner. The number of bits that represent coordinates of $P_{C_{j}}$ grows by at most 2 compared to $P_{C_{j-1}}$. It follows that $v_{m_{1}}$ requires at most $2 \mathrm{~m}$ bits more than $v_{1_{4}}$, which is still polynomial in $\mathrm{m}$. We set the $\mathrm{x}$-coordinate of $w_{4}$ to be equal to the $x$-coordinate of $v_{m_{1}}$. The $y$-coordinate of $w_{4}$ is 0 .

We are ready to discuss the construction of the spikes. Let us define an arrangements of lines as follows:

- For every clause $C_{j}, 1 \leqslant j \leqslant m$ that contains the literal $l_{q}, 1 \leqslant q \leqslant 3$ equal to the variable $u_{i}$, $1 \leqslant i \leqslant n$ create a pair of lines $L\left(x_{j_{q}}, f_{i_{10}}\right)$ and $L\left(a_{j_{q}}, f_{i_{6}}\right)$.

- For every clause $C_{j}, 1 \leqslant j \leqslant m$ that contains the literal $l_{q}, 1 \leqslant q \leqslant 3$ equal to $\overline{u_{i}}, 1 \leqslant i \leqslant n$ (the negation of the variable $\left.u_{i}\right)$ create a pair of lines $L\left(x_{j_{q}}, f_{i_{6}}\right)$ and $L\left(a_{j_{q}}, f_{i_{10}}\right)$.

- For every variable $u_{i}, 1 \leqslant i \leqslant n$ create a line $L\left(f_{i_{1}}, f_{i_{2}}\right)$.

Notice that $x_{j_{q}}$, for $1 \leqslant j \leqslant m, 1 \leqslant q \leqslant 3$ is an intersection point between $L\left(e_{j_{q}}, d_{j_{q}}\right)$ and $L\left(c_{j_{q}}, b_{j_{q}}\right)$. Both lines defined by the points with polynomial coordinates. Thus the number of bits required to represent the coordinates of $x_{j_{q}}$ is at most 5 times bigger then the number of bits in the coordinate representation of any of the four points. Thus the coordinates of $x_{j_{q}}$ are also polynomial in $m$.

Consider the T-well of the variable pattern for $u_{i}, 1 \leqslant i \leqslant n$ (refer to Fig. 22.5). Every line of the line arrangement that contains $f_{i_{10}}$ will define a spike in this well. We assume that a variable cannot participate in the same clause more than once. Thus the number of spikes in a particular well can be at most $\mathrm{m}$. We need to define a close neighbourhood in $P$ around $f_{i_{10}}$ from where the interior of every spike of the well will be visible. Extend the line segment $\overline{f_{i_{9}} f_{i_{10}}}$ towards $w_{1}$ until it first time hits a line in the arrangement (let $f^{\prime}$ be that intersection point). Let $f^{\prime \prime}$ be a midpoint of the line segment $\overline{f^{\prime} f_{i_{10}}}$. It is important to emphasize that we do not calculate lengths of line segments as a Euclidean distance between the points, because is involves the usage of a square root. To find the coordinates of $f^{\prime \prime}$ we first find the coordinates of $f^{\prime}$ (via the intersection of two lines defined by 4 points with polynomial coordinates). The $y$-coordinate of $f^{\prime \prime}$ is a half of the difference between the $y$-coordinate of $f^{\prime}$ and the $y$-coordinate of $f_{i_{10}}$. The $x$-coordinate of $f^{\prime \prime}$ is found in a similar way.

We can choose $m$ different points on the line segment $\overline{f^{\prime \prime} f_{i_{10}}}$ whose coordinates are polynomial in $m$. For every line $L$ in the arrangement that contains $f_{i_{10}}$ we do the following:

- If $L$ contains $a_{j_{q}}$ then create a line $L^{*}$ parallel to $L$ that passes through one of the $m$ points on $\overline{f^{\prime \prime} f_{i_{10}}}$ and intersects the line segment $\overline{a_{j_{q}} b_{j_{q}}}$.

- If $L$ contains $x_{j_{q}}$ then rotate L clockwise around $x_{j_{q}}$ to contain one of the $m$ points on $\overline{f^{\prime \prime} f_{i_{10}}}$. Create a line $L^{*}$ parallel to the updated $L$ such that it passes through $f_{i_{10}}$ and intersects the line segment $\overline{{\overline{j_{q}}}_{\mathrm{j}} b_{j_{q}}}$.

Every corresponding pair of lines L, L* defines a strip that in turn defines a spike. We choose the points on $\overline{f^{\prime \prime} f_{i_{10}}}$ to define the pairs $L, L^{*}$ in such a way that no two different strips overlap to the left of $L\left(f_{i_{7}}, f_{i_{8}}\right)$.

Notice that $\mathrm{L}$ is a line defined by a pair of points with polynomial coordinates; and $\mathrm{L}^{*}$ is a translation of $L$ by a vector with polynomial coordinates. Thus $L^{*}$ can also be defined via a pair of points with polynomial coordinates. Every spike contains 4 vertices. The coordinates of the two vertices of the spike that belong to $\overline{f_{i_{7}} f_{i_{8}}}$ are determined as the intersection points between $L\left(f_{i_{7}}, f_{i_{8}}\right), L$ and $L^{*}$. 
There are many polynomial choices in the intersection of the strip $L\left(f_{i_{7}}, f_{i_{8}}\right), L\left(f_{i_{6}}, f_{i_{5}}\right)$ with $L$ and $L^{*}$ for the other two vertices of the spike.

In a similar way we define the spikes in the F-well of the variable pattern for $u_{i}, 1 \leqslant i \leqslant n$. Notice that the coordinates of all the spikes in $\mathrm{P}$ are polynomial in $\mathrm{m}$.

We showed the construction of a simple polygon $P$ that has $49 m+10 n+3$ vertices such that the binary representation of the coordinates of those vertices is bounded by a polynomial in $\mathrm{n}$ and $\mathrm{m}$. Together with Lemma 22.5 this concludes the proof of Theorem 22.1. 
CONCLUDING REMARKS

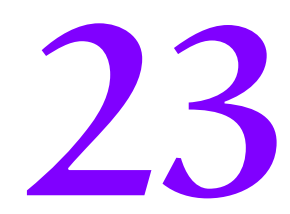

We showed that determining an optimal set of towers for trilateration of a simple polygon $\mathrm{P}$ is NPhard even if the complete information about $\mathrm{P}$ and the coordinates of all the towers are available to the point that wants to locate itself in the art gallery. To prove NP-hardness we showed a polynomial-time reduction from Boolean Three Satisfiability to our problem. In other words, we polynomially transformed a given instance of 3SAT into a simple polygonal region that can be trilaterated by $\mathrm{K}$ or fewer towers if and only if the 3SAT instance is satisfiable. Moreover, we proved that the reduction takes polynomial time. We demonstrated that the number of vertices of the constructed polygon is polynomial in the size of the given input instance of 3SAT and that the number of bits in the binary representation of the coordinates of those vertices is bounded by a polynomial in in the size of the input. 
BIBLIOGR A PHY

[1] Nina Amenta, Marshall W. Bern, and Manolis Kamvysselis. A new voronoi-based surface reconstruction algorithm. In Proceedings of the 25th Annual Conference on Computer Graphics and Interactive Techniques, SIGGRAPH 1998, Orlando, FL, USA, July 19-24, 1998, pages 415-421, 1998. doi: $10.1145 / 280814.280947$.

[2] Mark de Berg, Otfried Cheong, Marc van Kreveld, and Mark Overmars. Computational Geometry: Algorithms and Applications. Springer-Verlag TELOS, Santa Clara, CA, USA, 3rd ed. edition, 2008. ISBN 3540779736, 9783540779735.

[3] P. Bose, M. Mora, C. Seara, and S. Sethia. On computing enclosing isosceles triangles and related problems. Int. J. Comput. Geometry Appl., 21(1):303-318, 2011.

[4] Prosenjit Bose and Jean-Lou De Carufel. Minimum enclosing area triangle with a fixed angle. In CCCG, pages 171-174, 2010.

[5] Prosenjit Bose, Jean-Lou De Carufel, Alina Shaikhet, and Michiel H. M. Smid. Essential constraints of edge-constrained proximity graphs. In Combinatorial Algorithms - 27th International Workshop, IWOCA 2016, Helsinki, Finland, August 17-19, 2016, Proceedings, pages 55-67, 2016. doi: 10.1007/978-3-319-44543-4_5.

[6] Prosenjit Bose, Jean-Lou De Carufel, Alina Shaikhet, and Michiel H. M. Smid. Probing convex polygons with a wedge. Comput. Geom., 58:34-59, 2016. doi: 10.1016/j.comgeo.2016.06.001.

[7] Prosenjit Bose, Jean-Lou De Carufel, Alina Shaikhet, and Michiel H. M. Smid. Art gallery localization. submitted to Comput. Geom., 2017.

[8] Prosenjit Bose, Jean-Lou De Carufel, Alina Shaikhet, and Michiel H. M. Smid. Optimal art gallery localization is np-hard. submitted to Comput. Geom., 2017.

[9] Prosenjit Bose, Jean-Lou De Carufel, Alina Shaikhet, and Michiel H. M. Smid. Essential constraints of edge-constrained proximity graphs. J. Graph Algorithms Appl., 21(4):389-415, 2017. doi: 10.7155/jgaa.00422.

[10] Alfred M. Bruckstein and Michael Lindenbaum. Reconstruction of polygonal sets by constrained and unconstrained double probing. Ann. Math. Artif. Intell., 4:345-361, 1991.

[11] L. P. Chew. Constrained delaunay triangulations. Algorithmica, 4(1):97-108, 1989. doi: 10.1007/ BFo1553881.

[12] F. Chin and C. A. Wang. Finding the constrained delaunay triangulation and constrained voronoi diagram of a simple polygon in linear time. SIAM Journal on Computing, 28(2):471-486, 1998. doi: 10.1137/Soo97539795285916.

[13] V. Chvátal. A combinatorial theorem in plane geometry. Journal of Combinatorial Theory, Series B, 18(1):39-41, 1975. ISSN 0095-8956.

[14] R. Cole and C.K. Yap. Shape from probing. J. Algorithms, 8(1):19-38, 1987.

[15] Sanjoy Dasgupta, Christos H. Papadimitriou, and Umesh Vazirani. Algorithms. McGraw-Hill, Inc., New York, NY, USA, 1 edition, 2008. ISBN 0073523402, 9780073523408.

[16] O. Devillers, R. Estkowski, P.-M. Gandoin, F. Hurtado, P. A. Ramos, and V. Sacristán. Minimal set of constraints for $2 \mathrm{~d}$ constrained delaunay reconstruction. Int. J. Comput. Geometry Appl., 13 (5):391-398, 2003. doi: 10.1142/So218195903001244. 
[17] M. Dippel and R. Sundaram. An upper bound on trilaterating simple polygons. In CCCG, 2015.

[18] David P. Dobkin, Herbert Edelsbrunner, and Chee-Keng Yap. Probing convex polytopes. In STOC, pages $424-432,1986$.

[19] Herbert Edelsbrunner and Steven Skiena. Probing convex polygons with x-rays. SIAM J. Comput., $17(5): 870-882,1988$.

[20] S. Fisk. A short proof of Chvátal's watchman theorem. Journal of Combinatorial Theory, Series B, 24 (3):374 -, 1978. ISSN 0095-8956.

[21] R. Fleischer and Y. Wang. On the camera placement problem. In ISAAC, pages 255-264, 2009.

[22] K. R. Gabriel and R. R. Sokal. A new statistical approach to geographic variation analysis. Systematic Zoology, 18(3):pp. 259-278, 1969. ISSN 00397989. doi: 10.2307/2412323.

[23] R. J. Gardner. X-rays of polygons. Discrete Comput. Geom., 7:281-293, March 1992. ISSN 0179-5376.

[24] Michael R. Garey and David S. Johnson. Computers and Intractability: A Guide to the Theory of NP-Completeness. W. H. Freeman \& Co., New York, NY, USA, 1979. ISBN o716710447.

[25] $\mathrm{H}$ El Gindy and D Avis. A linear algorithm for computing the visibility polygon from a point. Journal of Algorithms, 2(2):186 - 197, 1981. ISSN 0196-6774.

[26] M. Hoffmann. Lecture notes in computational geometry, October 2009.

[27] J. W. Jaromczyk and M. Kowaluk. A note on relative neighborhood graphs. In SoCG, pages 233-241, 1987. ISBN o-89791-231-4. doi: 10.1145/41958.41983.

[28] J. W. Jaromczyk, M. Kowaluk, and F. Yao. An optimal algorithm for constructing $\beta$-skeletons in $l_{p}$ metric. ${ }^{1}$ Manuscript, 1989.

[29] B. Joe and R. B. Simpson. Corrections to lee's visibility polygon algorithm. BIT Numerical Mathematics, 27(4):458-473, 1987. ISSN 1572-9125.

[30] D. G. Kirkpatrick and J. D. Radke. A framework for computational morphology. In Computational Geometry, volume 2 of Machine Intelligence and Pattern Recognition, pages 217 - 248. North-Holland, 1985. doi: 10.1016/B978-0-444-87806-9.50013-X.

[31] D. T. Lee and A. K. Lin. Generalized dalaunay triangualtion for planar graphs. Discrete $\mathcal{E}$ Computational Geometry, 1:201-217, 1986. doi: 10.1007/BF02187695.

[32] D. T. Lee and A. K. Lin. Computational complexity of art gallery problems. IEEE Trans. Inf. Theor., 32(2):276-282, March 1986. ISSN 0018-9448. doi: 10.1109/TIT.1986.1057165.

[33] D.T Lee. Visibility of a simple polygon. Computer Vision, Graphics, and Image Processing, 22(2):207 $-221,1983$. ISSN 0734-189X.

[34] Shuo-Yen Robert Li. Reconstruction of polygons from projections. Inf. Process. Lett., 28(5):235-240, 1988.

[35] Veli Mäkinen, Simon J. Puglisi, and Leena Salmela, editors. Combinatorial Algorithms - 27th International Workshop, IWOCA 2016, Helsinki, Finland, August 17-19, 2016, Proceedings, volume 9843 of Lecture Notes in Computer Science, 2016. Springer. ISBN 978-3-319-44542-7. doi: 10.1007/ 978-3-319-44543-4.

1 This paper has been incorrectly referred as "to appear in SIAM J. Comput." in some other publications. 
[36] Henk Meijer and Steven S. Skiena. Reconstructing polygons from x-rays. Geometriae Dedicata, 61: 191-204, 1996. ISSN 0046-5755. 10.1007/BFo0151583.

[37] Joseph O'Rourke. Art Gallery Theorems and Algorithms. Oxford University Press, Inc., New York, NY, USA, 1987. ISBN o-19-503965-3.

[38] Anil S. Rao and Kenneth Y. Goldberg. Shape from diameter: Recognizing polygonal parts with a parallel-jaw gripper. I. J. Robotic Res., 13(1):16-37, 1994.

[39] Kathleen A. Romanik. Geometric probing and testing - a survey. Technical report, DIMACS, 95-42, September 1995.

[40] Steven S. Skiena. Geometric Probing. PhD thesis, University of Illinois, Urbana, IL, 1988.

[41] Steven S. Skiena. Problems in geometric probing. Algorithmica, 4(4):599-605, 1989.

[42] Steven S. Skiena. Probing convex polygons with half-planes. J. Algorithms, 12(3):359-374, 1991.

[43] D. D. Sleator and R. E. Tarjan. A data structure for dynamic trees. In STOC, pages 114-122. ACM, 1981. doi: $10.1145 / 800076.802464$.

[44] Csaba D. Tóth. Art gallery problem with guards whose range of vision is 180. Comput. Geom., 17 (3-4):121-134, 2000.

[45] G. T. Toussaint. The relative neighbourhood graph of a finite planar set. Pattern Recognition, 12 (4):261-268, 1980. doi: 10.1016/0031-3203(80)90066-7. 
COLOPHON

This document was typeset using the typographical look-and-feel classicthesis developed by André Miede. The style was inspired by Robert Bringhurst's seminal book on typography "The Elements of Typographic Style". classicthesis is available for both LTEX and LYX:

$$
\text { http://code.google.com/p/classicthesis/ }
$$

Happy users of classicthesis usually send a real postcard to the author, a collection of postcards received so far is featured here:

$$
\text { http://postcards.miede.de/ }
$$

Final Version as of August 8, 2017 (classicthes is version 4.1). 